\title{
Image Reconstruction for a Positron Emission Tomograph Optimized for Breast Cancer Imaging
}

\author{
by
}

\author{
Patrick Rommel Gonzalez Virador
}

B.A. (University of California at Berkeley) 1992

M.A. (University of California at Berkeley) 1994

A dissertation submitted in partial satisfaction of the

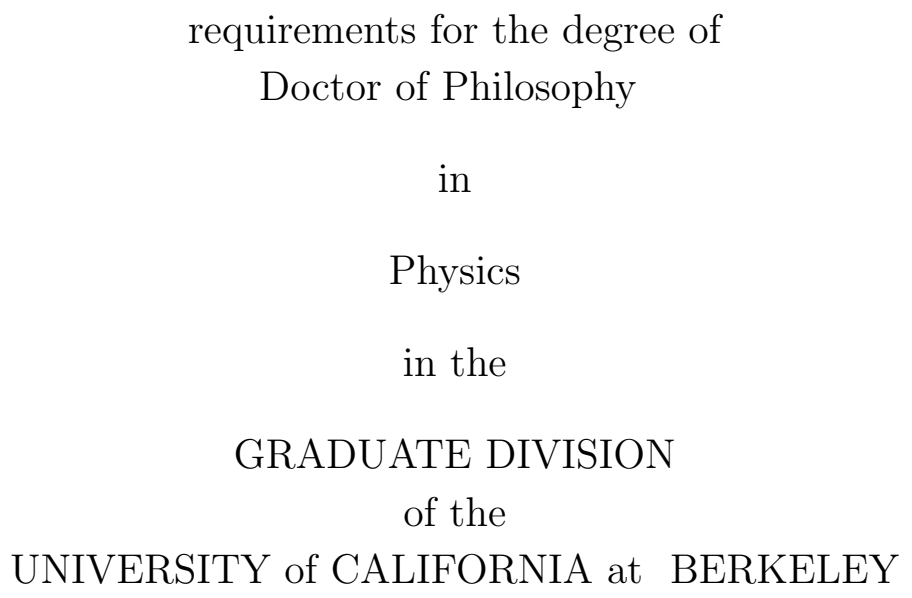

Committee in charge:

Professor Kam-Biu Luk and

Doctor William Moses, Cochairs

Professor Stephen Derenzo

Professor Marjorie Shapiro

Fall 2000 
The dissertation of Patrick Rommel Gonzalez Virador is approved:

\begin{tabular}{lr}
\hline Cochair & Date \\
\hline Cochair & Date \\
\hline & Date \\
\hline & \\
\hline
\end{tabular}

University of California at Berkeley

Fall 2000 


\title{
Image Reconstruction for a Positron Emission Tomograph Optimized for Breast Cancer Imaging
}

\author{
Copyright Fall 2000
}

by

Patrick Rommel Gonzalez Virador 


\author{
Abstract \\ Image Reconstruction for a Positron Emission Tomograph \\ Optimized for Breast Cancer Imaging \\ by \\ Patrick Rommel Gonzalez Virador \\ Doctor of Philosophy in Physics \\ University of California at Berkeley \\ Professor Kam-Biu Luk and \\ Doctor William Moses, Cochairs
}

I perform image reconstruction for a novel Positron Emission Tomography camera that is optimized for breast cancer imaging. This work addresses for the first time, the problem of fully-3D, tomographic reconstruction using a septa-less, stationary, (i.e. no rotation or linear motion), and rectangular camera whose Field of View (FOV) encompasses the entire volume enclosed by detector modules capable of measuring Depth of Interaction (DOI) information. The camera is rectangular in shape in order to accommodate breasts of varying sizes while allowing for soft compression of the breast during the scan. This non-standard geometry of the camera exacerbates two problems: a) radial elongation due to crystal penetration and b) reconstructing images from irregularly sampled data. Packing considerations also give rise to regions in projection space that are not sampled which lead to missing information. I present new Fourier Methods based image reconstruction algorithms that incorporate DOI information and accommodate the irregular sampling of the camera in a consistent manner by defining lines of responses (LORs) between the measured interaction points instead of rebinning the events into predefined crystal 
face LORs which is the only other method to handle DOI information proposed thus far. The new procedures maximize the use of the increased sampling provided by the DOI while minimizing interpolation in the data. The new algorithms use fixed-width evenly spaced radial bins in order to take advantage of the speed of the Fast Fourier Transform (FFT), which necessitates the use of irregular angular sampling in order to minimize the number of unnormalizable Zero-Efficiency Bins (ZEBs). In order to address the persisting ZEBs and the issue of missing information originating from packing considerations, the algorithms a) perform nearest neighbor smoothing in $2 \mathrm{D}$ in the radial bins b) employ a semi-iterative procedure in order to estimate the unsampled data and c) mash the "in plane" projections, i.e. 2D data, with the projection data from the first oblique angles, which are then used to reconstruct the preliminary image in the 3D Reprojection Projection algorithm. I present reconstructed images of point sources and extended sources in both $2 \mathrm{D}$ and 3D. The images show that the camera is anticipated to eliminate radial elongation and produce artifact free and essentially spatially isotropic images throughout the entire FOV. It has a resolution of $1.50 \pm 0.75 \mathrm{~mm}$ FWHM near the center, $2.25 \pm 0.75$ $\mathrm{mm}$ FWHM in the bulk of the FOV, and $3.00 \pm 0.75 \mathrm{~mm}$ FWHM near the edge and corners of the FOV.

Professor Kam-Biu Luk and

Doctor William Moses

Dissertation Committee Cochairs 
I dedicate this work to my heros, my parents,

Arnel and Cynthia.

A lifetime of thanks will not be enough... 


\section{Contents}

List of Figures vii

List of Tables $\quad$ xi

1 Introduction 1

1.1 Breast Cancer: Motivation . . . . . . . . . . . . . . . 1

1.2 Positron Emission Tomography and Oncology . . . . . . . . . . . . . . 3

1.2.1 Positron Emission Mammography _. . . . . . . . . . 5

1.3 Challenges in PEM Image Reconstruction . . . . . . . . . . . . . 5

1.3.1 Depth of Interaction and Radial Elongation . . . . . . . . . . 6

1.3.2 Non-standard Geometry . . . . . . . . . . . . . . . . 9

1.4 Outline . . . . . . . . . . . . . . . . . . 10

2 Image Reconstruction in PET $\mathbf{1 5}$

2.1 PET and Radon Transform . . . . . . . . . . . . . . . . 15

2.1.1 System Matrix . . . . . . . . . . . . . . . 15

2.1.2 Response Function . . . . . . . . . . . . . . . . . . . 17

2.1.3 Chord Distribution . . . . . . . . . . . . . . . . . . 21

2.1.4 System Matrix and Radon Transform . . . . . . . . . . . . . 23

2.2 Radon Transform and Its Inverse . . . . . . . . . . . . . . . . . . 26

2.2.1 Examples of Radon Transform . . . . . . . . . . . . . 26

2.2.2 Properties of Radon Transform . . . . . . . . . . . . . . 27

2.2.3 Inversion of Radon Transform . . . . . . . . . . . . . . . 30

2.2.4 Backprojection and Filtering . . . . . . . . . . . . . 32

2.3 Iterative Reconstruction Methods . . . . . . . . . . . . . . . . . 35

2.3.1 Discretization of the Problem . . . . . . . . . . . . 35

2.3.2 Objective Functions and Statistical Models . . . . . . . . . . . . 37

2.3.3 Pros and Cons of Iterative Reconstruction . . . . . . . . . . . 38 
3 The Camera $\quad \mathbf{4 1}$

3.1 Detector Module . . . . . . . . . . . . . . . . . 41

3.1 .1 Camera Geometry . . . . . . . . . . . . . . . 42

3.1.2 Previous attempts at correcting Radial Elongation . . . . . . . 43

3.1.3 Lutetium Orthosilicate . . . . . . . . . . . . . . . . . 46

3.1.4 Photodetectors . . . . . . . . . . . . . . . 4 48

3.2 Data Acquisition System . . . . . . . . . . . . . . . . . . . . . . . . . . . . . . . . . . . . . 49

3.2.1 Front End Electronics . . . . . . . . . . . . . . . . . 49

3.2 .2 Data Flow Control . . . . . . . . . . . . . . 50

3.3 Construction and Calibration . . . . . . . . . . . . . 54

4 Reconstruction in 2D $\quad 56$

4.1 Simulation . . . . . . . . . . . . . . . 56

4.1 .1 Phantom Simulation . . . . . . . . . . . . . . 56 56

4.1 .2 Photon Tracking . . . . . . . . . . . . . . . 58

4.1 .3 Detector Compton Scatter . . . . . . . . . . . . . . . 62

4.1.4 Simplified Camera . . . . . . . . . . . . . . 65

4.2 Reconstruction: Fourier Methods . . . . . . . . . . . . . . . 66

4.2 .1 Sampling . . . . . . . . . . . . . . . 66

4.2.2 Sinogram Parameters . . . . . . . . . . . . . . 70

4.2 .3 Reconstructed Images and Results . . . . . . . . . . . . . 74

4.3 Realistic Camera . . . . . . . . . . . . . . . . 76

4.3.1 Sampling Density and Sinogram Parameters . . . . . . . . . . 77

4.3.2 Reconstruction and Results . . . . . . . . . . . . . . 80

4.4 Summary of 2D Reconstruction Procedures . . . . . . . . . . . . 82

5 Reconstruction in 3D $\quad \mathbf{8 5}$

5.1 X-ray Transform . . . . . . . . . . . . . . . . . . . . 88

5.2 Filtered Backprojection in 3D and Colsher's Filter . . . . . . . . . . . 92

5.2.1 Truncation: Missing Information . . . . . . . . . . . . 95

5.2.2 Kinihan and Rogers Algorithm (3DRP) . . . . . . . . . . . 97

5.3 Normalization in $\mathbf{R}^{3} \ldots \ldots \ldots \ldots$. . . . . . . . . . . . . . 99

5.4 Polar Acceptance . . . . . . . . . . . . . . . . . . . . . . . 101

5.4.1 Signal-to-Noise Measurements: Parameters and Algorithms . . 105

5.4.2 Signal-to-Noise Measurements: Results . . . . . . . . . . . . 111

5.5 Polar Sampling . . . . . . . . . . . . . . . . . . 115

5.5.1 Resolution Test . . . . . . . . . . . . . . 119

5.5.2 Normalization Revisited . . . . . . . . . . . . . . . . . . . 122

5.6 A Sample Reconstruction . . . . . . . . . . . . . . . . . 127 
5.7 Realistic Camera Geometry . . . . . . . . . . . . . . . . . 127

5.7 .1 Reconstructing Initial 2D Estimate . . . . . . . . . . . . 133

5.7.2 Final 3D Reconstruction . . . . . . . . . . . . . . . 141

5.8 Summary of 3D Reconstruction Procedures . . . . . . . . . . . . . 143

6 Reconstructed Images $\quad 146$

6.1 Flood Phantom . . . . . . . . . . . . . . . . . . . . 146

6.2 Point Sources . . . . . . . . . . . . . . . . . . . . 147

6.2.1 Profiles Phantom . . . . . . . . . . . . . . . . 147

6.2 .2 Noise Phantom . . . . . . . . . . . . . . . . . 150

6.2.3 Derenzo-like Point Sources . . . . . . . . . . . . . . . 151

6.3 Extended Source Phantoms . . . . . . . . . . . . . . . . . . . 152

6.3.1 Defrise Phantom . . . . . . . . . . . . . . . 152

6.3 .2 Spheres and Ellipsoids . . . . . . . . . . . . . . 152

7 Conclusions and Future Directions $\quad 174$

$\begin{array}{ll}\text { A Fourier Rebinning Method } & 177\end{array}$

$\begin{array}{ll}\text { Bibliography } & 180\end{array}$ 


\section{List of Figures}

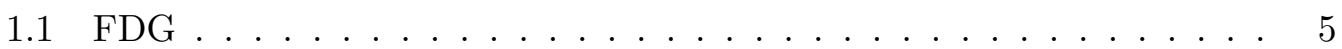

1.2 Radial Elongation . . . . . . . . . . . . . . . . 7

2.1 Variables used to determine System Matrix. . . . . . . . . . . . . 16

2.2 Pulse and Trapezoidal Functions. The axes of the plots are arbitrary. . 17

2.3 Pictorial representation of the probability photon detection. . . . . . . 18

2.4 Response Function A. . . . . . . . . . . . . . . . . . . . . . 20

2.5 Response Functions B and C. . . . . . . . . . . . . . . 21

2.6 Examples of Chord Distributions . . . . . . . . . . . . . . . . 24

2.7 Integral of Response Function. . . . . . . . . . . . . . . . 25

2.8 Coordinate System Used for Radon Transform. . . . . . . . . . . . . . . 25

2.9 Sample Objects. . . . . . . . . . . . . . . . . . . 27

2.10 Radon Transforms of Sample Objects. . . . . . . . . . . . . . . . 28

2.11 Central Slice Theorem in 2D. . . . . . . . . . . . . . . 30

$2.12 r \phi$ as Rectilinear Variables . . . . . . . . . . . . . . . . . 33

2.13 Backprojection of $1,2,4$, and $\infty$ projections. . . . . . . . . 34

2.14 Models used to calculate System Matrix elements . . . . . . . . . . . . 39

3.1 The PEM Module . . . . . . . . . . . . . . . . . . . 47

3.2 Schematic of one channel of charge sensitive amplifier . . . . . . . . 50

3.3 Data flow and processing in the Analog Subsection . . . . . . . . . . 52

3.4 Electronics organization for the PEM data acquisition system. . . . . . 53

3.5 DOI Measurement Set-up . . . . . . . . . . . . . . . . . . 55

4.1 Example of generation of limited polar angle, $\theta \ldots \ldots \ldots$

4.2 Histogram of generated scattering angle as a function of energy . . . . 60

4.3 Photoelectric Fraction and Linear Attenuation Coefficient of LSO . . . 61 
4.4 Compton Kinematics. The $x$ axis are in radians while the $y$ axis is relative probability. . . . . . . . . . . . . . 62

4.5 Simplified PEM Geometry with one module plane. . . . . . . . . . . 65

4.6 Definition of Chords with and without DOI. . . . . . . . . . . . . 67

4.7 Sampling Density without DOI information. . . . . . . . . . . 68

4.8 Sampling Density with DOI information. Regions (A), (B), and (C) are discussed in text. . . . . . . . . . . . . . 6 68

4.9 Sampling Density with DOI information in Region (B) of Fig. 4.8. . . 72

4.10 Angular Binning Scheme. . . . . . . . . . . . . . . . . . . 73

4.11 PSF Reconstruction. . . . . . . . . . . . . . . . . . . . . 74

4.12 Realistic PEM Geometry with one module plane. . . . . . . . . . . . . 76

4.13 Sampling density of realistic camera . . . . . . . . . . . 78

4.14 Sampling density of realistic camera after DOI smoothing . . . . . . 79

4.15 Reconstructed point sources within the realistic camera . . . . . . . 80

4.16 Reconstructed extended sources within the realistic camera . . . . . . 82

5.1 Wireframe image of idealized PEM Detector Geometry . . . . . . . . . 87

5.2 Coordinate System Used for 3D X-ray Transform. . . . . . . . . . . 89

5.3 Projections of the FOV at various directions. . . . . . . . . . . . 90

5.4 Definition of Colsher Polar acceptance angle $\psi$. . . . . . . . . . . . 93

5.5 Truncated projections of the FOV at various directions . . . . . . . . 96

5.6 Fraction of truncated bins in each projection plane . . . . . . . . . . 97

5.7 Distributions of Normalization values, for 2D and 3D . . . . . . . . . 100

5.8 Fraction of excluded bins for various normalization limits. . . . . . . 102

5.9 Histogram of Fraction of excluded bins for various normalization limits.103

5.10 Angular Distribution of Detected Events. . . . . . . . . . . . . . . . 104

5.11 Cumulative Polar Distribution of Detected Events. . . . . . . . . . . 105

5.12 Regions in FOV . . . . . . . . . . . . . . . . . . . . . 110

5.13 Signal-to-noise ratio of a $5 \times 5$ pixel array centered at $0 \mathrm{~A}, 1 \mathrm{~A}, 2 \mathrm{~A}$, and 3A as shown in Fig. 5.12 . . . . . . . . . . . . . . . . . . 112

5.14 Signal-to-noise ratio of a $10 \times 10$ pixel array centered at $0 \mathrm{~A}, 1 \mathrm{~A}, 2 \mathrm{~A}$

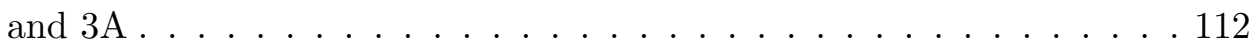

5.15 Signal-to-noise ratio of a $15 \times 15$ pixel array centered at $0 \mathrm{~A}, 1 \mathrm{~A}, 2 \mathrm{~A}$

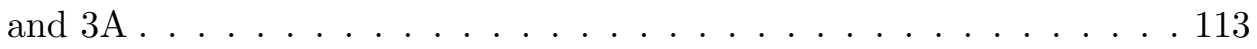

5.16 Signal-to-noise ratio of a $15 \times 15$ pixel array centered at $0 \mathrm{~B}, 1 \mathrm{~B}$ and $2 \mathrm{~B} 113$

5.17 Extended Phantom used to verify Polar Acceptance. . . . . . . . . . 115

5.18 Signal-to-noise ratio of Flood and Phan001 phantoms . . . . . . . . . 116

5.19 Signal-to-Noise ratio of flood phantoms comparing fine and coarse sampling schemes in polar angle $\theta$. . . . . . . . . . . 118 
5.20 Middle plane of Point Sources Phantom. . . . . . . . . . . . . . . . . 120

5.21 Point Sources carved out by algorithm as described in text. The axes indicate the voxel indices. . . . . . . . . . . . . . . 120

5.22 Comparison of Fine and Coarse Polar angle sampling using Point Sources. . . . . . . . . . . . . . . . . . . . . 123

5.23 Comparison of ideal means. . . . . . . . . . . . . . . . 124

5.24 Ratio of $E\left[\frac{1}{\lambda_{i}}\right]$ and $\frac{1}{E\left[\lambda_{i}\right]}$ if $\lambda_{i}$ is Poisson distributed. . . . . . . . . . 125

5.25 Mean voxel value as a function of plane index after Poisson correction 126

5.26 Mean voxel value as a function of plane index after including more events in the normalization file . . . . . . . . . . . . . . 126

5.27 Coarse Polar Sampling Reconstruction of Ellipse Extended Source Phantom. The axes indicate the pixel indices in the plane. . . . . . . 128

5.28 Plane statistics of reconstructed extended ellipse phantom. . . . . . . . 129

5.29 Wireframe image of realistic PEM Detector Geometry . . . . . . . . . 130

5.30 Energy spectrum of detected events . . . . . . . . . . . . . . . 131

5.31 Difference between true depth and calculated depth for the resolution conditions in Fig. 5.30 . . . . . . . . . . . . . . . . . . 132

5.32 Depth estimator $\Gamma$ and redistributed DOI . . . . . . . . . . . 133

5.33 Projections of FOV at various directions . . . . . . . . . . . . 134

5.34 Mashed Projections . . . . . . . . . . . . . . . 135

5.35 Redistributed Projections. . . . . . . . . . . . . . . . 136

5.36 Normalized Projections. . . . . . . . . . . . . . . . . . 137

5.37 Filled Projections. . . . . . . . . . . . . . . . . . . 138

5.38 Sum of pixel differences between first and subsequent iterations for projection at $\phi=\frac{\pi}{2}$. The plot is zero suppressed and therefore does not show the first iteration. . . . . . . . . . . . . . . 138

5.39 Mean voxel value and standard deviation of reconstructed flood source after the 5 th iteration of IRR. . . . . . . . . . . . . . . . . 139

5.40 Reconstructed Flood Source after the 5th iteration of IRR. . . . . . . 140

5.41 Fraction of filled bins in each projection plane . . . . . . . . . . . . . 142

6.1 Pictorial representation of methods of splitting events in the problem-

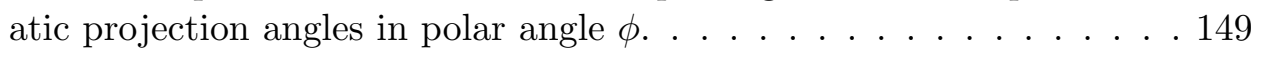

6.2 3D Reconstruction of Flood Source . . . . . . . . . . . . . . . . . . 159

6.3 Image statistics of Flood Phantom. . . . . . . . . . . . . . . . . . 160

6.4 Point Sources Identification Numbers . . . . . . . . . . . . . . . 160

6.5 Planes 47 through 55 of Profiles Phantom. . . . . . . . . . . . . . 161

6.6 Profiles through Plane 51 of Profiles Phantom. . . . . . . . . . . . . 162

6.7 Planes 63, 75 and 87 of Profiles Phantom. . . . . . . . . . 163 
6.8 Planes 95 through 103 of Profiles Phantom. . . . . . . . . . . . . . 164

6.9 Profiles through Plane 99 of Profiles Phantom. . . . . . . . . . . . . 165

6.10 Planes 49 through 57 of Noise Phantom. . . . . . . . . . . . . 166

6.11 Planes 89 through 97 of Noise Phantom. . . . . . . . . . . . . . . 167

6.12 Planes 48 through 56 of Derenzo-like point sources. . . . . . . . . . . . 168

6.13 Planes 90 through 98 of Derenzo-like point sources. . . . . . . . . . . . 169

$6.14 x$ slices of reconstructed Defrise Phantom . . . . . . . . . . . . . 170

$6.15 y$ slices of reconstructed Defrise Phantom . . . . . . . . . . . . . 171

6.16 Mean Voxel Value and Standard Deviation of a $3 x 3$ voxel ROI region at the center of each quadrant of the Defrise Phantom as a function of plane number. . . . . . . . . . . . . . . . . . . 172

6.17 Mean Voxel Value and Standard Deviation of a $15 \times 15$ voxel ROI region at the center of each quadrant of the Defrise Phantom as a function of plane number. . . . . . . . . . . . . . . . 172

6.18 Reconstructed Spheres and Ellipsoids. . . . . . . . . . . . . . . 173 


\section{List of Tables}

3.1 Scintillator Properties of NaI, BGO, and LSO . . . . . . . . . . . 46

4.1 Results of algorithm optimization for determining detector Compton scatter ........................... 63

4.2 PSF Resolution for Various DOI Resolution. . . . . . . . . . . . . . 75

4.3 PSF Resolution for Various DOI Resolution. . . . . . . . . . . . . . . . 81

5.1 Geometric Acceptance of Detectors. . . . . . . . . . . . . . . 86

5.2 Polar projection angles and widths. . . . . . . . . . . . 106

5.3 A. Azimuthal projection angles and widths. . . . . . . . . . . . . 108

5.4 B. Azimuthal projection angles and widths. . . . . . . . . . . . . 109

6.1 FWHM of point sources in the middle plane of the FOV in Profiles Phantom. . . . . . . . . . . . . . . . 155

6.2 FWHM of point sources in planes between the middle and top planes of Profiles Phantom. . . . . . . . . . . . . . . . . . 156

6.3 FWHM of point sources near the top plane of the FOV of Profiles Phantom. . . . . . . . . . . . . . . . 157

6.4 Volumetric Extent of the reconstructed point sources in the Noise Phantom. . . . . . . . . . . . . . . . . . . 158

6.5 Variation in integrated point source activity and peak values of point sources in Noise Phantom . . . . . . . . . . . . . . . . . . 158 


\section{Acknowledgements}

I will now add to the infinite number of acknowledgments that begin with "There are too many people to thank..." This dissertation is the result of many people's ideas, suggestions, comments, corrections, redirections, encouragements, comforting, coaching, coaxing, and diversions.

I would like to begin this litany of names with two instructors in high school who inspired me tremendously. I will not be getting off this train now if they had not brought me to the station many years ago. Mr. Jacobo Gonzales, my Algebra instructor, showed me mathematics in nature, and taught me, with a lot of patience, why $2 x$ is never the answer to "What is $x * x ? "$ I rarely make that mistake now. Ms. Maribel Abear, my Biology instructor, showed me the wonders of science, and taught me how to see beauty in nature, even in the slugs that we kept in our Biology lab aquaria. I only hope she has forgiven me for studying Physics instead of Biology.

I met a wonderful mentor in Prof. Kam-Biu Luk. By my senior year in college, I was enamored by the Theory of High Energy Physics and was begrudgingly taking Physics 111 Advanced Lab, not understanding why it was important for me to learn experimental techniques. Kam-Biu taught me that a scientist needs to understand not only theory, but how to test the theories with experiments because experiments determine whether the theories are any good. Working with him the summer after I graduated convinced me to study Experimental High Energy physics in graduate school. I would not have passed my Preliminary Exams without his help. However, my study of High Energy Physics was short lived and I decided to leave the field to pursue another avenue of study after my stay at Fermilab in Illinois. I hoped that Kam-Biu was not disappointed with my decision to leave HEP. He continued to be encouraging, helping me change my study to Medical Imaging by being my "in house" advisor and supporting my petition to the Physics Department to allow

me to pursue my new area of research. I needed an "in house" advisor because no one in the department studied Medical Imaging. There was, however, someone at 
the Lawrence Berkeley National Lab's Center for Functional Imaging who agreed to take me on as his student.

Dr. Bill Moses was to be my advisor from then on. Bill was ever mindful of my progress as a student, always aware, it seemed to me, to know just when to let me do my work and when to call me into his office to talk about what's going on. He has kept me focused even when I have, on many occasions, insisted on doing everything that was to detract me from my task. He has always been level with me, making sure I knew my options, and allowing me to make the choices. I have learned a lot from Bill: from working with integrity to how to deal diplomatically with almost everyone. He was always available when I was having difficulties juggling grad school and everything else, specially during the times when I swore I was never going to finish. He has always argued that I've done all the work whenever I have expressed my gratitude to him, specially when I passed my quals and when I spoke with him to tell him that I was ready to leave (not in so many words). While it may be true that I wrote the code, ran the simulations, and reconstructed the images, Bill gave me every opportunity, all facilities, and overwhelming support to do everything I that have done. All his future students should consider themselves extremely fortunate.

I will always cherish the company and friendship of everyone at the Center for Functional Imaging, specially the members of the Instrumentation Group. The many cookies that Jenny Huber brought to the lab are the least of the things for which I need to thank her. The many times Greg Gruber and Matt Ho pulled me from my terminal to take a break and walk up to Building 90 for a snack have, I'm certain, at one time or another, prevented my brain from shutting down from exhaustion. Matt has also been a good friend, gracious enough to share his wisdom that goes beyond his years. For the many questions that they have answered, and the many interesting conversations and exchanges, I would like to thank Jinyi Qi, Ron Huesman, Greg Klein, Bryan Reutter, Jonathan Maltz, Martin Boswell, and Dr. Tom Budinger.

I would like to express special thanks to Profs. Marjorie Shapiro and 
Stephen Derenzo for serving as members in my qualifying exam and dissertation committees. They have also been my guides in graduate school: Marjorie during my earlier years in High Energy Physics and Steve in my later years in Medical Imaging.

The Physics Department Student Services staff is a great group of people who have made my stay at Cal pleasant and enjoyable. I am certain I have tested Donna Sakima's patience when I've asked her, semester after semester, for my enrollment ID number. She has had to put up with me since I was an undergraduate. Anne Takizawa has been helpful in innumerable ways, from informing me what I needed during the time I was petitioning the Department for my research field, to when Spieker Pool would be open to students whenever it closed. And I would like to thank Claudia Trujillo for steering students who needed tutoring in physics into my direction. Any extra income is always welcome to a graduate student.

This acknowledgment will be incomplete if I do not mention my friends. I would like to thank Erich Varnes. We started on the road to HEP together and I would like to thank him for the many many good hikes, movies, grub, challenging games of Star Wars on Nintendo, and the times I needed to copy the solution of a problem in a homework set. Wait, that's for the times that I needed to discuss a solution to a homework. Yeah, that's it. I would like to thank Matthew Baker as well, for his insistence that I needed to leave the lab on occasion, if only to see a natural source of light. Many thanks too to Sam and Karon Stradley, Jamie and Mike Wilson, Dan Durkin, Laura Lising, Mary Rowe, Ernie Simmons and Eric Winter. Their kind words and deeds made the journey fun. I would like to say thanks to a special friend. She hasn't answered in words the many times I've asked her for guidance, but I've heard her in many other ways. Dolores, thank you.

My learning and curiosity began at home, and so I would like to thank my family. None of what I have done would have been possible without their support. My brother Peter has always shown me new ways to look at old and difficult problems. Having worked through the academic system herself, my sister-in-law Vicky has always given me good advice on how to approach situations. And of course, I would 
like to thank my parents. My father and I didn't talk too much about my work, but I knew that he has always considered my happiness as his own. And my mom, who assured me that I could have her backyard to build another accelerator when the funding for the SSC was cut, was always encouraging, never expecting me to do anything but what I wanted. To the heros of my life, I'd like to say thanks.

And finally, I wish I can say that mere words are not enough to thank Ms. Jennifer Rush, but I know I will never hear the end of it if I did. It is rather curious that I got to know Jennifer almost at the beginning of my research in Medical Imaging, as if Providence knew that I was going to need a lot of help. And help she has been. She has been my inspiration to start a new task, my encouragement when the task became difficult, my comfort when I failed, and my joy when I succeeded. If there was ever a measure of patience, it should be her patience with me. I just hope I didn't make her wait too long. 


\section{Chapter 1}

\section{Introduction}

\subsection{Breast Cancer: Motivation}

Breast cancer is the leading cause of cancer deaths in women in the United States. Estimates of new cases of breast cancer range from 90,000 to 182,000 each year, indicating that approximately $8 \%$ of the women born in the United States will develop this disease [1][2]. Various studies have shown that mass screening for breast cancer with x-ray mammography has decreased the rate of mortality in women by $30 \%$, and as much as $70 \%$ in women over 50 [3]. The low cost of planar x-ray mammography (less that $\$ 100$ ) coupled with its $100 \mu \mathrm{m}$ resolution make it an effective method for mass screening.

The Positron Emission Mammography (PEM) project was initially conceived to complement x-ray mammography and assist in the treatment and management of breast cancer. Originally, the project had three goals:

- to determine whether nuclear medicine techniques can be used as an alternative to biopsy when suspicious lesions are observed in a mammogram,

- to determine whether a Positron Emission Tomography (PET) camera can be used to asses the involvement of the axillary nodes if breast cancer is diagnosed 
- to build a PET camera that can monitor the progress of the disease and determine the efficacy of treatment

The first motivation of this project is to determine whether PET and nuclear medicine techniques can be used as an alternative to biopsy. X-ray mammography is a structural imaging modality that measures the attenuation of an x-ray beam as it passes through the breast. While its resolution allows for the detection of submillimeter calcifications that are tell-tale signs of breast cancer, the malignancy of aggregate masses in the breast that show no microcalcifications are difficult to assess based solely on their appearance on x-ray film. In particular, this problem is acute in cases of radiologically dense breasts that may have regions which appear as suspicous masses but are otherwise normal tissue. In fact, $20 \%-80 \%$ of masses seen in mammograms are benign [2]. Therefore, the detection of a suspicious lesion in a mammogram is often followed by a biopsy to determine malignancy of the mass.

There are many reason why biopsy is undesirable. It is time consuming and extremely uncomfortable for the patient. Hard compression of the breast is necessary during the incision of the needle and throughout the entire extraction of the tissue to be examined. The continuous stereotactic x-ray fluoroscopy required for guiding the needle in the breast significantly increases the radiation dose to the patient. Tumors are missed approximately $10 \%$ of the time. The scarring that may occur as a result of the biopsy may make subsequent mammograms difficult to interpret because scar tissue often has different radiological properties from normal tissue. The camera discussed in this thesis will be able to determine the malignancy of a suspicious mass in the breast and may obviate the need for biopsy.

The second motivation for this project is to build a camera that may be used for the treatment and management of breast cancer in the unfortunate circumstance that the disease is diagnosed. Axillary node involvement is an indicator of the extent of the disease and thus plays a major role in determining the best treatment of the disease. The prognosis is favorable if the cancer has not metastisized to the axillary 
nodes which are located at the outside edges of the breast near the armpits. It is extremely unfavorable otherwise. Currently, the only course of action to determine the health of the nodes are excision and biopsy since imaging the structure of the nodes is insufficient to determine whether the cancer has metastisized to the sites. The nodes may be enlarged for reasons other than the invasion of malignant tissue and so it is important to determine their biochemical properties. The removal of the nodes often cause bad side effects. The optimizations that are done on the PEM camera for imaging the breast make it ideal for imaging the axillary nodes.

The final motivation for the project was to build a camera that could aid monitor the progress and treatment of breast cancer. In the event that surgery becomes necessary, scar tissue may further hinder interpretation of further x-ray mammograms. The optimized camera could continue to image the breast and scan for malignant tissue in spite of the scarring.

\subsection{Positron Emission Tomography and Oncology}

PET is a medical imaging technique that measures the biochemical activity of tissue, represented by the local concentration of a radiotracer injected into the patient. Radiotracers are biologically active and radioactively labeled drugs that are engineered to study and image specific tissue and biochemical processes [4]. In PET, these radiotracers are positron emitters $\left(\beta^{+}\right.$decay). The ejected positron traverses a short distance in the tissue, depending on the radiotracer used, and forms positronium with an electron. The two particles annihilates via $e^{+} e^{-} \rightarrow$ $\gamma \gamma$, producing two nearly back-to-back $511 \mathrm{keV}$ photons ${ }^{1}$, in accordance with the conservation of energy and momentum. These photons are detected by a pair of radiation detectors that constrain the location of the radiotracer to be along the line connecting them. This line is called a line of response (LOR) or a chord. The collection of activated chords constitute the data acquired in a PET scan that are

\footnotetext{
${ }^{1}$ The positron annihilates before themalization.
} 
subsequently reconstructed into an image.

The use of PET scanners in oncology for the study and evaluation of tumors is among the fastest growing areas in nuclear medicine [5]. The success of PET in imaging glucose uptake in the brain, in particular in regions of the brain where increased metabolism is observed as a result of performing tasks (e.g. speech), encouraged many radiologists to apply the method in detection of tumors, which, as evidenced by their rapid growth, also have increased metabolism. The promise of improved resolution by up to a factor of five over its single photon analog (Single Photon Emission Computed Tomography or SPECT) and the possibility of more accurate quantitation have contributed to the excitement of applying PET in oncology.

Increased glucose uptake is among the characteristics of tumor cells. There are many reasons proposed for this phenomenon and this is an active area of research in Oncology; but chief among these reasons is the Pasteur Effect: the facilitation of glucose metabolism in cells by the lack of oxygen. While investigating the fermentation process in winemaking, Louis Pasteur observed that cells consumed more glucose in the absence of oxygen. The reason for this phenomenon is that while complete aerobic glycolysis produces 36 molecules of ATP, the energy source for cells, anaerobic glycolysis produces only two molecules of ATP [6]. Thus, cells need more glucose to sustain their energy requirements in anaerobic environments. The fast growth of tumor cells often outpaces the production of new blood vessels resulting in an oxygen starved environment. Warburg confirmed the occurance of the Pasteur Effect in cancerous tissue in 1931 [7].

The radiotracer ${ }^{18} \mathrm{~F}$-flouro-2-deoxyglucose (FDG) is the primary reason behind the recent interest in PET for clinical application in oncology [8]. FDG (Fig. 1.1) is a sugar analog where a hydroxyl group $\mathrm{OH}$ is replaced by ${ }^{18} \mathrm{~F}$, a positron emitter. The location of the ${ }^{18} \mathrm{~F}$, in the 2-position in the carbon ring, prohibits the conversion of the glucose into glycogen, allowing FDG to accumulate in the cell. This mechanism makes FDG more "sticky" than either ${ }^{11} \mathrm{C}$-labeled or ${ }^{15} \mathrm{O}$-labeled compounds which are also used in PET in oncology. 


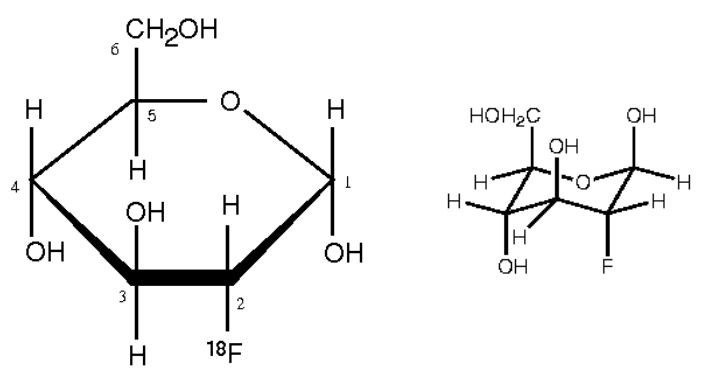

Figure 1.1: FDG. ${ }^{18}$ F-flouro-2-deoxyglucose (FDG) is the primary reason behind the recent interest in $\mathrm{PET}$ for clinical application in oncology.

\subsubsection{Positron Emission Mammography}

Positron Emission Mammography (PEM) is the application of PET in the diagnosis, treatment and management of breast cancer. Several other PEM cameras have been proposed in recent years which, like our own camera, are optimizations of conventional PET design. These include the MAMPET [9] and the McGill University PEM [10]. The latter camera is designed to fit into an X-ray mammography unit. It is composed of two banks of PET modules, one attached to the base of the mammogram unit while the other to the X-ray tube. It cannot perform tomographic reconstruction because it does not completely surround the breast. The requirement of a complete set of projections for tomographic reconstruction is discussed in Chapter 4. Interest in applying PET in breast cancer has been fueled by reports that uptake of FDG in breast tumors relative to normal tissue can be as high as 8:1 [11].

\subsection{Challenges in PEM Image Reconstruction}

The optimization that we have done in order to successfully use PET in mammography lead to challenges in image reconstruction. There are primarily two main issues that need to be addressed: a) the inclusion of the Depth of Interaction 
(DOI) information into the reconstruction algorithm, and b) the non-conventional geometry of the detector. The camera's geometry require fully-3D reconstruction because it will not have septa. The camera's large axial extent complicates this matter as I shall discuss in Chapter 5 . In addition, there will be gaps between detector modules that will prevent the camera from sampling some regions in projection space. In this thesis, I address these challenges by developing algorithms that can be used to reconstruct images from data acquired with the PEM camera. My goal is to reconstruct qualitatively sound images from a simulated PEM camera and as such, a major component of this disertation is the modeling of the camera via Monte Carlo simulation. I will characterize the reconstructed images semi-quantitatively to serve as a guide for future work in trying to extract quantitative information from the camera. It is my hope that these algorithms will be used when the construction of the camera is completed.

\subsubsection{Depth of Interaction and Radial Elongation}

Radial Elongation is the degradation of image resolution caused by the penetration of the $511 \mathrm{keV}$ photons into crystals. It is the result of associating events with the incorrect LOR's. Fig. 1.2 (A) shows how penetration leads to mispositioning of events and thus degradation of resolution. Annihilation A is associated with the correct LOR while annihilation B is not (dotted line). The anticipated Point Spread Functions (PSF), the shapes of the reconstructed point sources located at radiotracers $\mathrm{A}$ and $\mathrm{B}$, are beneath the tomograph ring. Note that event mispositioning occurs only when the photons enter the crystals obliquely, worsening with increasing angle between the photon and the long axis of the crystal. For circular tomographs, pointsource resolution can be degraded by up to a factor of three at distances of half the radius of the camera where an incident photon can penetrate the crystals at $\frac{\pi}{6}$ radians $[12]$.

Measuring the DOI information is essential to reconstructing artifact free 


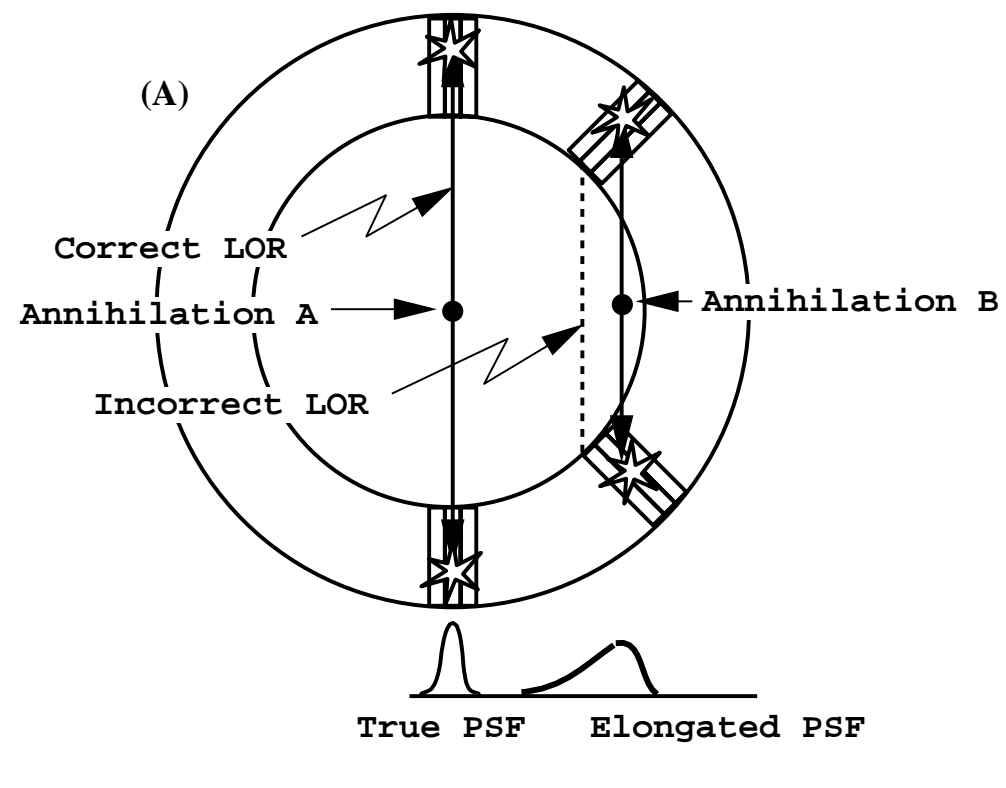

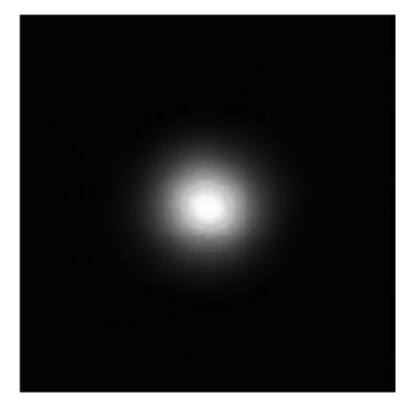

(B)

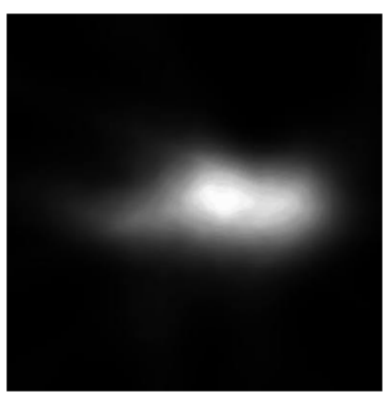

(C)

Figure 1.2: Radial Elongation. Resolution of PET cameras degrade away from the center of the tomograph. (B) is a reconstructed point source at the center of a 20.0 $\mathrm{cm}$ radius tomograph while $(\mathrm{C})$ is a reconstructed point source at $r=10.0 \mathrm{~cm}$. 
images for the PEM camera. In order to achieve greater detector sensitivity over conventional PET cameras, the detector modules are packed as closely as possible to the breast being imaged. Without DOI, radial elongation will prohibitively degrade the resolution away from the center of the camera, resulting in varying resolution in the FOV and causing blurred reconstructed images. The DOI information eliminates radial elongation. Figs. $1.2(\mathrm{~B})$ and (C) are reconstructions of point sources at $r=10$ $\mathrm{cm}$ of a $20 \mathrm{~cm}$ radius tomograph. Image (B) is reconstructed with DOI information while image $(\mathrm{C})$ is reconstructed without DOI information.

Radial blurring is particularly acute in the PEM camera because the breast will occupy the entirety of the field of view (FOV). Conventional PET cameras minimize blurring by limiting the useful FOV to regions close to the center of the camera where the effect of crystal penetration is small. A FOV that is half the tomograph's radius is common. With the rectangular geometry of the PEM camera, we expect that at least a third of the photons detected will have penetrated the crystals at angles $>\frac{\pi}{6}$ radians.

While there have been several attempts at detector designs that measure DOI information [13][14][15], little work has been done to determine the best way to incorporate the DOI information into the reconstruction algorithm. Moses et. al [16] have proposed rebining the LOR measured with DOI into LORs that are measured without DOI information. While this procedure partially corrects penetration ${ }^{2}$ it does not take advantage of the increased sampling provided by the DOI. A DOI resolution of $\sigma_{D O I}$ effectively increases the number of LOR by $\left(\frac{L_{\text {crystal }}}{\sigma_{D O I}}\right)^{2}$, i.e. half a crystal length DOI resolution quadruples the number of LOR. I will discuss a method of incorporating DOI information that uses this increased spatial sampling. It minimizes the interpolation that is required if the events were merely rebined. The incorporation of the DOI information into the image reconstruction is one of the contributions of this thesis to the field of medical imaging.

\footnotetext{
${ }^{2}$ Rebinning associates the true chord with the closest chord that can be defined by crystal faces
} 


\subsubsection{Non-standard Geometry}

Another challenge in reconstructing images from data taken from the PEM camera originates from the non-standard geometry of the camera. In order to maximize the sensitivity of the camera, it is packed as tightly as possible to minimize gaps between modules. This is achieved by packing the modules into a rectangular camera. The shape of the camera also accomodates breasts of varying sizes and allows for the soft compression of the breast during the scan. Compression prevents the breast from moving which would result in motion artifacts in images.

While there are several advantages to having a rectangular camera, its shape breaks the circular symmetry assumed by Fourier Image Reconstruction methods. The modules of the camera sample the object in the FOV irregularly, making the image reconstruction difficult at best. The extension of the FOV to include the entire region enclosed by the camera modules excacerbates the irregular sampling. Conventional tomographs with irregular sampling rebin the data into a regular samples by interpolation. Interpolation of data to force regular sampling in function reconstruction generally gives poor results [17]. Note that even if the camera were circular, the extension of the FOV will still result in irregular sampling of the object. This is due to the finite widths of the crystals in the detector modules. The spacing between the LOR decreases as chords farther and farther from the center are considered. Indeed, this is another reason why conventional tomographs limit the FOV to regions close to the center where sampling is approximately regular.

Another challenge in reconstructing images from the PEM camera is the fact that the camera will be without septa. Septa are used to reduce scattered radiation and so preferentially accept events that lie within a predefined plane of the detector. This is done so that a three dimensional image can be reconstructed by stacking 2D images that can be quickly reconstructed. The use of septa, however, greatly decreases the acceptance and sensitivity of the camera. The absence of septa in the camera necessitates the use of fully-3D reconstruction. Standard algorithms 
which are used for fully-3D reconstruction assume cameras with small axial extent, commonly $\theta \approx .25$ radians. The PEM camera has over twice this axial extent, rendering approximate algorithms unusable.

Finally, due to packing limitations, the PEM camera will have gaps between the detector modules. These gaps will cause portions of the projection space to be unsampled making reconstruction nearly impossible. Cameras that have gaps in their modules [18] have unsampled projection-space locations that are contiguous, making them easier to deal with. In comparison, the unsampled locations in the PEM projection space are scattered about the entire space.

\subsection{Outline}

The work that I describe in this dissertation, other than the description of the hardware and the simulation of the camera, can be thought of as finding a transfer function between "bad data space" and "reconstructable data space." I use standard Filtered Backprojection algorithms that are modified to address the particular idiosyncracies of the camera caused by its non-standard geometry and its capability to measure DOI information. As such, the chapters containing the bulk of the novel works that I have done are structured in the following way: a) I discuss the standard procedures that would be used if the PEM camera were a standard PET machine; b) describe why these procedures, if left unmodified, will produce artifacts in the reconstructed images; c) discuss the modifications needed to ensure artifact-free images.

This thesis is outlined as follows:

- Chapter 2 - Image Reconstruction in PET: I present a novel view of the connection between the data acquisition process in PET and the Radon Transform, whose inversion is the basis for the theory of analytic image reconstruction. This view introduces a new mathematical formalism for the System Matrix by expressing it as the product of the Response Function of the camera, 
the basis for Natural Pixel Formulation of Image Reconstruction [19], and the Chord Distribution which I define as the set of chords between two detectors in projection space. It is widely assumed that the Line Integral formulation of PET data is the System Matrix taken to some limit, and I suggest a formalism by which this limit can indeed be viewed as the limit of infinitely small detectors. While I focus on Fourier Inversion methods, I give a discussion of iterative methods in $2 \mathrm{D}$ for completeness.

- Chapter 3 - The Camera: I describe the components of the detector as well as how it is contructed. I begin by discussing the new scintillator we use, Cerium-doped Lutetium Orthosilicate (LSO), and some of its properties. I then discuss the photodectors, the construction of a module, and the electronics for data acquisition and processing. The measurement of the DOI information is essential to the success of the camera as an imaging device; I explain how it is obtained.

- Chapter 4 - Reconstruction in 2D: I begin the investigation of reconstruction algorithms for the camera by limiting the dimensionality of the problem to $2 \mathrm{D}$. While the camera is operated only in fully $3 \mathrm{D}$-mode, $2 \mathrm{D}$ images can be reconstructed by considering only events that are coplanar within the crystal planes (an example of software collimation). This allows me to consider the various sampling issues that needed to be addressed without the complications of fully 3D image reconstruction. In this chapter, I describe the simulation with which I generate the events and data sets that I reconstruct.

The PEM camera violates key assumptions used in standard filtered backprojection techniques, namely regular sampling of both radial and angular variables. In order to take full advantage of the DOI capability of the camera, which greatly increases the sampling of the FOV in all but the $\phi=0, \frac{\pi}{2}$ directions, I use fine linear sampling. Fine linear sampling produces ZEBs that result in 
gross streak artifacts in the reconstructed images. These ZEBs are minimized if irregular angular sampling is employed. In order to reconstruct artifact-free images from PET data whose angular variable is irregularly sampled, I have developed procedures that accomodate the irregular angular sampling. These procedures involve multiplying the filtered data by a factor that is proportional to the bin width of the angular samples. The scaled data are backprojected around the center of the projection angle a number of times that is determined by the ratio of the width of the angular bin to the minimum angular bin width. The chapter culminates with $2 \mathrm{D}$ images reconstructed with these new procedures and their characterization.

- Chapter 5 - Reconstruction in 3D: This chapter extends the $2 \mathrm{D}$ reconstruction techniques developed in Chapter 4 into 3D. I describe the X-Ray transform and how 3D reconstruction is done with conventional cameras. I discuss issues regarding normalization, the optimizations that are needed for the succesful 3D reconstruction of images with the PEM camera, and the new techniques that are used in order to address problems that have not surfaced in the $2 \mathrm{D}$ reconstruction.

In this chapter, I develop procedures by which the "useful" axial extent and necessary polar sampling of any 3D camera can be determined by using signal to noise ratios of reconstructed images. Using these procedures, I determine a useful axial extent for the camera that is large enough to prohibit the use of more conventional fast 3D reconstruction techniques. Therefore, I employ the standard but slower 3DRP reconstruction algorithm. However, as in the case of $2 \mathrm{D}$ reconstruction with the PEM, it is necessary to modify the standard algorithm in order to ensure that the reconstructed images are artifact free. The modifications involve a mashing of the "in-plane" data with the first oblique projections. This is neccessary so that the techniques that I have 
developed in Chapter 4 can be used to estimate the premilinary 2D image from which the reprojected data is calculated. The sampling parameters that I have previously determined to be essential for high resolution reconstruction necessitates another new procedure in the normalization of the reprojected data. In essence, the reprojected data is "denormalized" so that it can be properly compared with the acquired data to determine the scale factor between them. Finally, I show that the missing data resulting from a) the truncation due to incomplete angular coverage ${ }^{3}, \mathrm{~b}$ ) the gaps between modules, and c) the definition of $511 \mathrm{keV}$ hits to be at the center of DOI bins, can be addressed consistently by treating all of them as trucated data to be estimated by the properly scaled reprojection data.

- Chapter 6 - Reconstructed Images: This chapter shows reconstructions of simulated phantoms scanned by a simulated "physical" PEM detector, i.e. with realistic energy and DOI resolutions. I give results of image resolution and noise characterization studies on the images. The chapter culminates with an extended source phantom made up of ellipsoids just as the chapter on $2 \mathrm{D}$ reconstruction techniques.

- Chapter 7 - Conclusions and Future Directions: I conclude with closing remarks, a summary of the results, a list of the limitations of and possible future directions for this work.

- Appendix A - The Fourier Rebinning Method: The Exact Fourier Rebinning Method (FOREX) is a promising algorithm that may be used to reconstruct images for the PEM camera. I believe that it will be able to consistently address many of the issues that make analytical reconstruction of data from the PEM camera problematic. While its forerunner algorithm, the FORE algorithm, became available during my investigation, it required key assumptions

\footnotetext{
${ }^{3}$ The PEM camera, for obvious reasons, cannot completely enclose the breast.
} 
that are not applicable for the PEM camera. The FOREX algorithm did not become available until the end of my search for reconstruction algorithms for the PEM camera. Both of these algorithms rebin the "off plane" events into the "in plane" projections and reconstruct the 3D object by stacking planes of $2 \mathrm{D}$ reconstructed images. I discuss the theoretical basis for the algorithm in this appendix. 


\section{Chapter 2}

\section{Image Reconstruction in PET}

In this chapter, I introduce the Radon Transform and discuss its relation to data acquired in PET. I discuss several of its properties that are useful for the inversion and image reconstruction, and elucidate its relation to the Fourier transform. Finally, I introduce the Central Slice Theorem which is essential in the inversion of the Radon Transform. The inversion of the Radon Transform is the basis of the Fourier Inversion Methods of Medical Imaging. Most of the formulation and notation used in this chapter are expressedly 2D, but generalizing into higher dimension (3D in particular) is not too difficult. The inversion of the Radon Transform, however, will be in $n$ dimensions, in anticipation of the following chapter.

\subsection{PET and Radon Transform}

\subsubsection{System Matrix}

The data collected during a PET scan is a realization of the application of a functional to the distribution of radionuclides in the object being scanned. Consider the data recorded from a PET event: the line connecting a pair of detectors simultaneously hit by $511 \mathrm{keV}$ photons. This line can be parametrized by $s$, its perpendicular distance from the origin, and $\phi$, the angle it makes with the $x$-axis. 

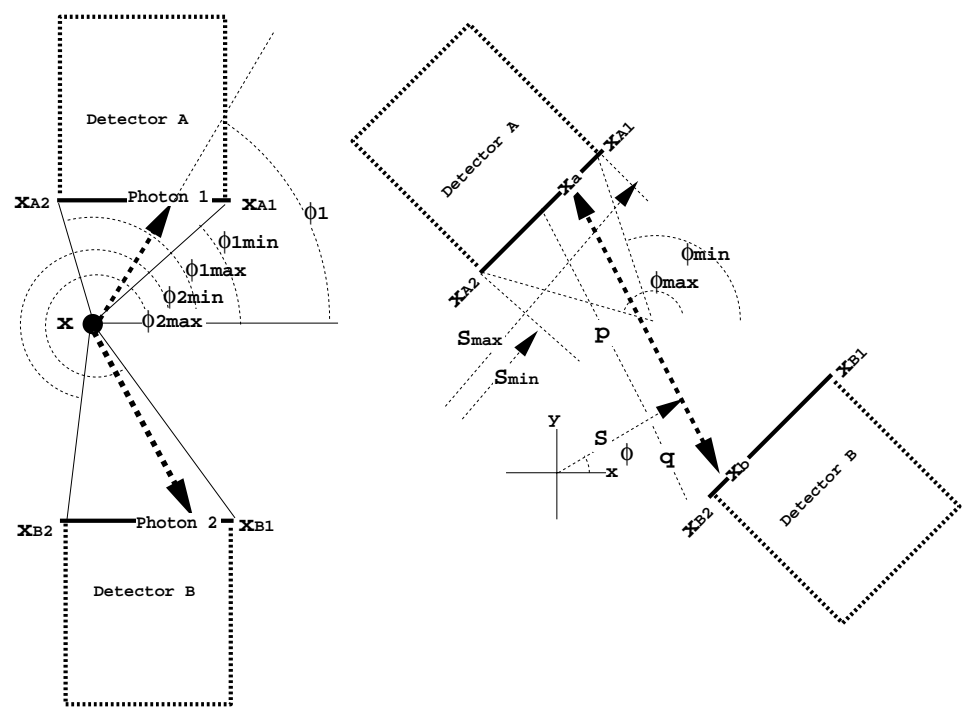

Figure 2.1: Variables used to determine System Matrix.

I will denote $\mathbf{s}=(s, \phi)$. The complete data set is the collection of such hits. Let $D$ be a set of points in coordinate space, and let $P(\mathbf{x}, \mathbf{s}) d^{2} s d^{2} x$ be the probability that an annihilation within $d^{2} x$ of $\mathbf{x} \in D$ is detected within $d^{2} s$ of the parameters $\mathbf{s}$ in Radon space. The origin of the name of this space will become clear below. We can associate $D$ with the support of objects within the FOV of a PET camera. Note that because objects in PET scans have finite extents, $D$ is compact, i.e. it is closed and bounded [20]. Thus, given $\lambda(\mathbf{x})$ as the concentration of the radionuclide tracer, the complete PET data set is then

$$
S(\mathbf{s}) d^{2} s=\int_{D} d^{2} x P(\mathbf{x}, \mathbf{s}) \lambda(\mathbf{x}) d^{2} s
$$

$P(\mathbf{x}, \mathbf{s})$ is called the System Matrix and is unique to each PET camera system. We can then interpret PET data as the range of the functional $\int_{D} d^{2} x P(\mathbf{x}, \mathbf{s})$.

In order to determine $P(\mathbf{x}, \mathbf{s})$, I need to calculate a) the probability that an annihilation is detected by a pair of detectors and b) the region defined by the pair of detectors in Radon space. The former can be calculated by measuring the solid 


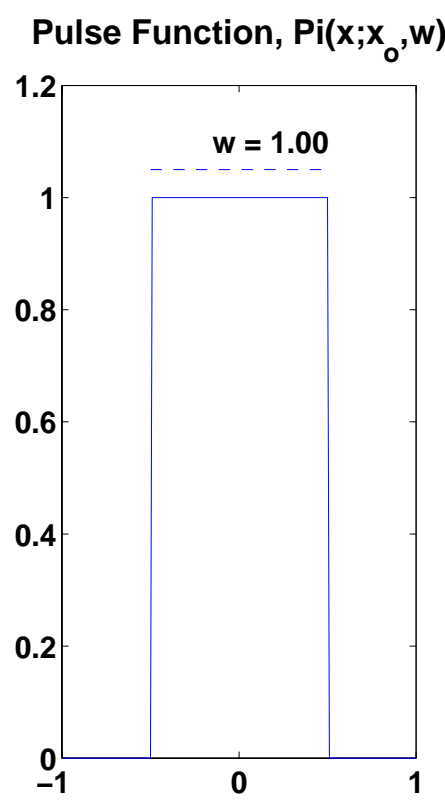

\section{Trapezoidal Function, $\mathbf{T}\left(\mathbf{x} ; \mathbf{x}_{\mathbf{0}}, \mathrm{t}, \mathrm{b}\right)$}

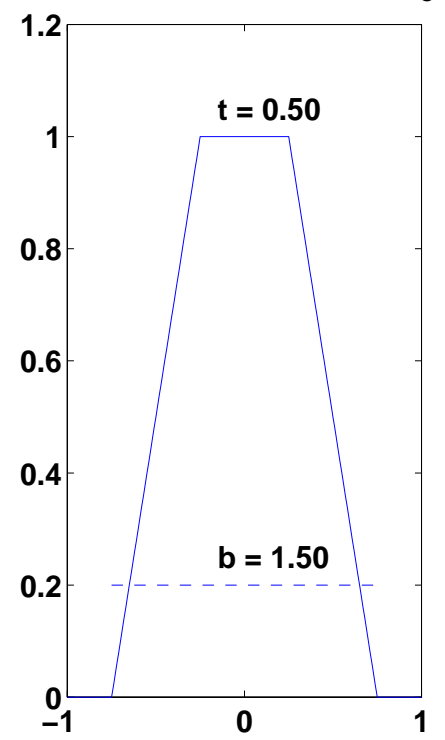

Figure 2.2: Pulse and Trapezoidal Functions. The axes of the plots are arbitrary.

angles (2D) subtended by the detectors as seen from the annihilation point. The latter can be calculated by a change of variables from the coordinate variables $x y$ to the Radon variables $s \phi$.

\subsubsection{Response Function}

First, I calculate the probability that an annihilation at $\mathbf{x}$ is detected by a pair of detectors. This is called the Response Function. Fig. 2.1 shows two detectors where the faces are highlighted as opposed to the bulk. I consider only the detector face. The bulk can be thought of as a stack of detector faces. Let $\mathbf{x}$ be the location of the annihilation. Assuming small angle approximation, i.e. $\sin \phi=\phi$, the probability that photon 1 hits the face of detector $\mathrm{A}$ is independent of its incidence angle, i.e.

$$
R_{A-1}\left(\mathbf{x} ; \mathbf{x}_{A 1}, \mathbf{x}_{A 2}\right)=\frac{1}{2 \pi} \int_{0}^{2 \pi} d \phi_{1} \Pi\left(\phi_{1} ; \frac{\phi_{1}^{\max }+\phi_{1}^{\min }}{2}, \phi_{1}^{\max }-\phi_{1}^{\min }\right)
$$




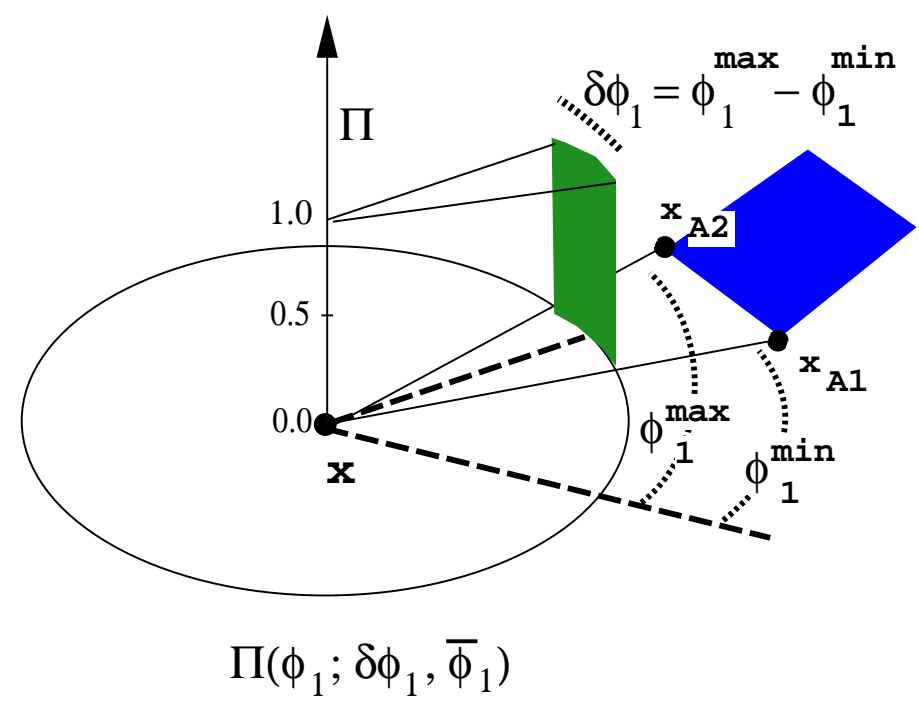

Figure 2.3: Pictorial representation of probability photon detection. The probability is a Pulse function in $\phi$ with a width given by the difference $\delta \phi_{1}=\phi_{1}^{\max }-\phi_{1}^{\min }$ and the center by the median $\bar{\phi}=\frac{\phi_{1}^{\min }+\phi_{1}^{\max }}{2}$. Note that these angles are implicit functions of $\mathbf{x}, \mathbf{x}_{A 1}$, and $\mathbf{x}_{A 2}$.

where $\Pi$ is the pulse function (or the rectangular function) centered at $x_{o}$ with width. $\Pi$ is given by

$$
\Pi\left(x ; x_{o}, w\right)= \begin{cases}1 & \text { if }\left|x-x_{o}\right|<\frac{w}{2} \\ 0 & \text { otherwise }\end{cases}
$$

and is shown in Fig. 2.2. Note that $R_{A-1}\left(\mathbf{x} ; \mathbf{x}_{A 1}, \mathbf{x}_{A 2}\right)$ measures the fraction of $2 \pi$ that is between $\mathbf{x}_{A 1}$ and $\mathbf{x}_{A 2}$ as seen from $\mathbf{x}$, i.e. it is the normalized probability of detector A detecting photon 1 .

Recalling that the angles $\phi_{1}^{\text {min }}$ and $\phi_{1}^{\max }$ are implicit functions of $\mathbf{x}, \mathbf{x}_{A 1}$, and $\mathbf{x}_{A 2}$, I will use the following analogy to further describe $R_{A-1}$. At each point $\mathbf{x}$ in the space containing detector $\mathrm{A}$, there is a unit circle whose center is $\mathbf{x}$. Consider a ray from $\mathbf{x}$. If this ray intersects the face of detector $\mathrm{A}$, i.e. the line segment $\left(\mathbf{x}_{A 1}, \mathbf{x}_{A 2}\right)$, then the unnormalized probability that a photon emitted in this direction will be detected is 1 . Otherwise, the unnormalized probability is 0 . In Fig. 2.3, this is 
depicted pictorally. In $\phi$ space, the probability is a Pulse function between $\phi_{1}^{\text {min }}$ and $\phi_{1}^{\max }$. The width of this Pulse function is the difference $\phi_{1}^{\max }-\phi_{1}^{\min }$, while the center of the function is the median angle $\frac{\phi_{1}^{\min }+\phi_{1}^{\max }}{2}$. Thus, the unnormalized probability that detector A detects photon 1 is given by the area under a Pulse function in the polar space $\phi$, as given in Eqn. 2.2.

Likewise, the probability that photon 2 is detected by detector B is given by a similar expression, i.e. it is also a pulse function with the width $\phi_{2}^{\max }-\phi_{2}^{\min }$ and center $\frac{\phi_{2}^{\min }+\phi_{2}^{\max }}{2}$. We can express the correlation between the photon 1 and photon 2 by the constraint $\delta\left(\phi_{2}-\left(\phi_{1}+\pi\right)\right)$.

The joint probability that photon 1 hits detector A and photon 2 hits detector $\mathrm{B}$ is therefore given by

$$
\begin{aligned}
R_{A-1, B-2}\left(\mathbf{x} ; \mathbf{x}_{A 1}, \mathbf{x}_{A 2}, \mathbf{x}_{B 1}, \mathbf{x}_{B 2}\right)= & \frac{1}{2 \pi} \int_{0}^{2 \pi} d \phi_{1} \Pi\left(\phi_{1} ; \frac{\phi_{1}^{\text {max }}+\phi_{1}^{\text {min }}}{2}, \phi_{1}^{\text {max }}-\phi_{1}^{\text {min }}\right) \\
& \Pi\left(\phi_{1}+\pi ; \frac{\phi_{2}^{\max }+\phi_{2}^{\text {min }}}{2}, \phi_{2}^{\text {max }}-\phi_{2}^{\text {min }}\right) .
\end{aligned}
$$

I have already applied the $\delta$-function constraint on the second $\Pi$ function in the integrand.

Note that Eqn. 2.4 is of the form

$$
\left.\int d x f(x) g(x-y)\right|_{y=-\pi}
$$

which is the convolution of two functions evaluated at $\phi_{1}=-\pi$. In our case, these two functions are both Pulse functions. The convolution of two $\Pi$ functions is the Trapezoidal Function $T\left(x ; x_{o}, t, b\right)^{1}$ such that $\Pi\left(x ; c_{1}, w_{1}\right) \otimes \Pi\left(x ; c_{2}, w_{2}\right)=T\left(x ; c_{1}-\right.$ $\left.c_{2},\left|w_{2}-w_{1}\right|, w_{2}+w_{1}\right) . T$ is defined as

$$
T\left(x ; x_{o}, t, b\right)= \begin{cases}\frac{2}{b}(x-1) & \text { if } x_{o}-\frac{b}{2}<x<x_{o}-\frac{t}{2} \\ 1 & \text { if }\left|x-x_{o}\right|<\frac{t}{2} \\ \frac{-2}{b}(x-1) & \text { if } x_{o}+\frac{t}{2}<x<x_{o}+\frac{b}{2} \\ 0 & \text { otherwise }\end{cases}
$$

\footnotetext{
${ }^{1}$ The parameters $x_{o}, t, b$, of $T$ correspond to its center, the extent of its maximum value, and its support, respectively. The letters $t$ and $b$ are mnemonics for "top" and "bottom."
} 


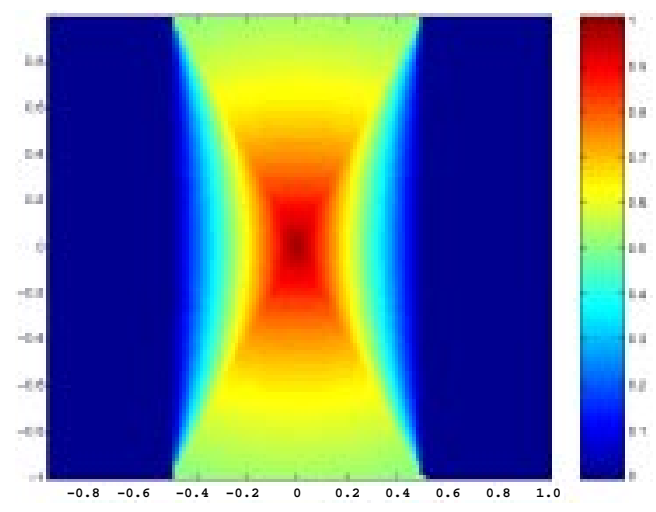

Figure 2.4: Response Function A.

Fig. 2.2 plots a trapezoidal function. Thus $R_{A-1, B-2}(\mathbf{x}) \propto T$ with $x_{o}=\phi_{1}^{a v g}-\phi_{2}^{a v g}$, $t=\left|\delta \phi_{2}-\delta \phi_{1}\right|$ and $b=\delta \phi_{2}+\delta \phi_{1}$. Therefore, the joint probability that photon 1 is seen by detector A and photon 2 is seen by detector B is the Trapezoidal function with the appropriate paramters evaluated at $\phi=\pi$.

Fig. 2.4 shows an example of a response function for the detector pair $\mathbf{x}_{A} \in\{|x|<0.5, y=1.0\}$ and $\mathbf{x}_{B} \in\{|x|<0.5, y=-1.0\}$. It is normalized such that the maximum value is 1 . The function is a pulse function near the detectors and is triangular function midway between them. The height of the triangle is twice the height of the pulse. The function is trapezoidal inside the region defined by the detector faces and zero everywhere else. The trapezoids are oriented perpendicular to the line connecting the front faces of the detectors. Fig. 2.5 shows two other examples of the response function: (B) is for the detector pair $\mathbf{x}_{A} \in\{|x+0.5|<$ $0.5, y=-1.0\}$ and $\mathbf{x}_{B} \in\{x=1.0,|y-0.5|<0.5\}$, while $(\mathrm{C})$ is for the detector pair $\mathbf{x}_{A} \in\{|x-0.5|<0.5, y=-1.0\}$ and $\mathbf{x}_{B} \in\{x=1.0,|y+0.5|<0.5\}$. Note that the geometry of the detector pair for response function $(\mathrm{C})$ breaks the small angle approximation near the corner. 

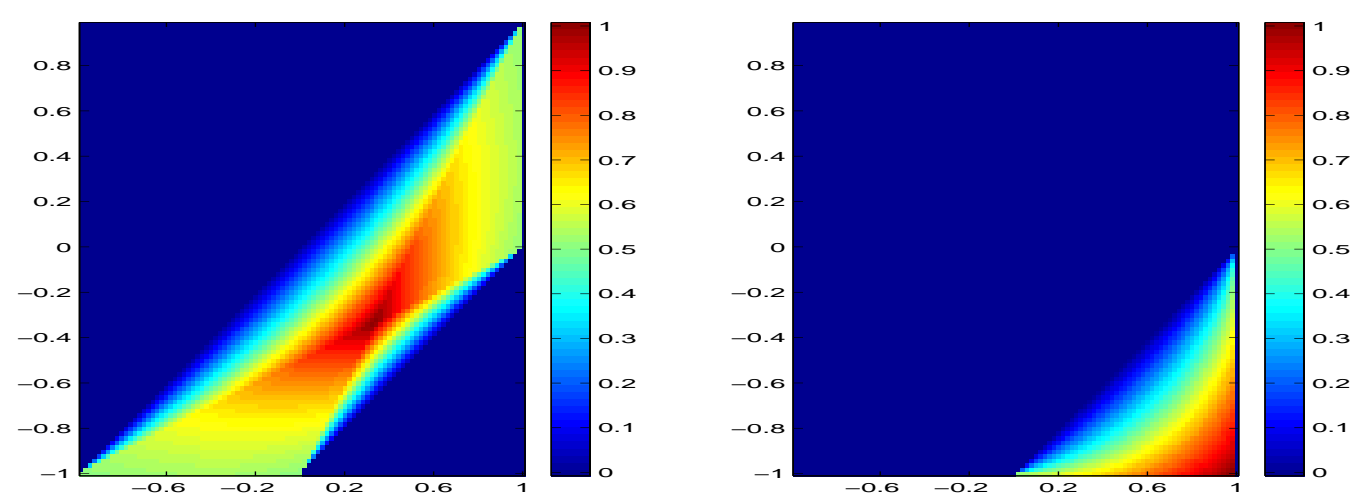

Figure 2.5: Response Functions B and C.

\subsubsection{Chord Distribution}

I now determine the region in Radon space, i.e. the parameter space $s \phi$, defined by a pair of detectors. This distribution $C$ is called the Chord Distribution. Let $\Psi_{A}\left(\mathbf{x}_{a}\right)$ and $\Psi_{B}\left(\mathbf{x}_{b}\right)$ define a pair of detectors such that

$$
\Psi_{i}\left(\mathbf{x}_{i}\right)= \begin{cases}1 & \text { if } \mathbf{x}_{i} \in\left\{\mathbf{x}_{I}\right\}, \text { i.e. Detector I } \\ 0 & \text { otherwise }\end{cases}
$$

Thus the distribution of lines of response defined by detectors A and B is

$$
C_{A, B}\left(\mathbf{x}_{a}, \mathbf{x}_{b}\right)=\Psi_{A}\left(\mathbf{x}_{a}\right) \Psi_{B}\left(\mathbf{x}_{b}\right)
$$

I need to write this distribution in terms of $s$ and $\phi$. From Fig. 2.1, I can write the following:

$$
\begin{aligned}
\phi & =\frac{\pi}{2}+\tan ^{-1}\left(\frac{y_{a}-y_{b}}{x_{a}-x_{b}}\right) \\
s & =y_{a} \sin \phi+x_{a} \cos \phi=y_{b} \sin \phi+x_{b} \cos \phi \\
p & =-x_{a} \sin \phi+y_{a} \cos \phi \\
q & =-x_{b} \sin \phi+y_{b} \cos \phi
\end{aligned}
$$


Therefore

$$
\begin{aligned}
C_{A, B}(\mathbf{s}) & =\int_{R} \int_{R} d p d q C_{A, B}(\mathbf{s}, p, q) \\
& =\int_{R} \int_{R} d p d q C_{A, B}\left(\mathbf{x}_{a}, \mathbf{x}_{b}\right)\left|\frac{\partial\left(\mathbf{x}_{a}, \mathbf{x}_{b}\right)}{\partial(\mathbf{s}, p, q)}\right|
\end{aligned}
$$

Since

$$
\left[\begin{array}{l}
x_{a} \\
y_{a}
\end{array}\right],\left[\begin{array}{l}
x_{b} \\
y_{b}
\end{array}\right]=\left[\begin{array}{cc}
\cos \phi & -\sin \phi \\
\sin \phi & \cos \phi
\end{array}\right]\left[\begin{array}{l}
s \\
p
\end{array}\right],\left[\begin{array}{l}
s \\
q
\end{array}\right]
$$

the Jacobian is

$$
\left|\frac{\partial\left(\mathbf{x}_{a}, \mathbf{x}_{b}\right)}{\partial(\mathbf{s}, p, q)}\right|=|p-q|
$$

The Convolution Theorem ${ }^{2}$ allows us to write any function as integrals over $\delta$ functions so I consider the case when

$$
\begin{aligned}
D_{A}\left(x_{a}, y_{a}\right) & =\delta\left(x_{a}-\chi_{a}\right) \delta\left(y_{a}-\gamma_{a}\right) \\
D_{B}\left(x_{b}, y_{b}\right) & =\delta\left(x_{b}-\chi_{b}\right) \delta\left(y_{b}-\gamma_{b}\right)
\end{aligned}
$$

This is the limiting case of infinitely small detectors. Thus

$$
\begin{aligned}
C_{A, B}^{\delta}(\mathbf{s})= & \int_{R} \int_{R} d p d q|p-q| \\
& \delta\left(s \cos \theta-p \sin \theta-\chi_{a}\right) \delta\left(s \sin \theta+p \cos \theta-\gamma_{a}\right) \\
& \delta\left(s \cos \theta-q \sin \theta-\chi_{a}\right) \delta\left(s \sin \theta+q \cos \theta-\gamma_{a}\right)
\end{aligned}
$$

Using the properties of the $\delta$-function

$$
\begin{aligned}
\delta(\alpha x) & =\frac{\delta(x)}{|\alpha|} \\
\delta(f(x)-a) & =\sum_{i} \frac{\delta\left(x-x_{i}\right)}{\left.\left|\frac{d f}{d x}\right|\right|_{f\left(x_{i}\right)=a}}
\end{aligned}
$$

\footnotetext{
${ }^{2}$ The Convolution Theorem is also known as the Sifting Property of the Dirac $\delta$-function, i.e. $f(x)=\int d y f(y) \delta(y-x)$
} 
I get

$$
C_{A, B}^{\delta}(\mathbf{s})=\left|\frac{\chi_{a}-\chi_{b}}{\sin \phi}\right| \delta\left(s-\chi_{a} \cos \phi-\gamma_{a} \sin \phi\right) \delta\left(s-\chi_{b} \cos \phi-\gamma_{b} \sin \phi\right)
$$

From the first $\delta$-function, $s=\chi_{a} \cos \phi+\gamma_{a} \sin \phi$, so I can rewrite the second $\delta$ function as

$$
\begin{aligned}
& \delta\left(s-\chi_{b} \cos \phi-\gamma_{b} \sin \phi\right)=\frac{\delta(m+\cot \phi)}{|\sin \phi|\left|\chi_{a}-\chi_{b}\right|} \\
& m=\frac{\chi_{b}-\gamma_{b}}{\chi_{a}-\gamma_{a}}
\end{aligned}
$$

to get

$$
\begin{aligned}
C_{A, B}^{\delta}(\mathbf{s}) & =\frac{1}{|\sin \phi|^{2}} \delta\left(s-\chi_{a} \cos \phi-\gamma_{a} \sin \phi\right) \delta(m+\cot \phi) \\
& =\delta\left(s-\chi_{a} \cos \phi-\gamma_{a} \sin \phi\right) \delta\left(\phi-\arctan (m)+\frac{\pi}{2}\right)
\end{aligned}
$$

Fig. 2.6 shows the chord distribution for the three detector pairs discussed in Figs. 2.4 and 2.5. The distribution was calculated by assuming that each point on each detector is equally probable to be hit. Of course in a real PET system, this probability is modified by the response function integrated over all the points in the FOV.

\subsubsection{System Matrix and Radon Transform}

The System Matrix can therefore be written as

$$
\begin{aligned}
& P\left(\mathbf{x}, \mathbf{s} ; \mathbf{x}_{a 1}, \mathbf{x}_{a 2}, \mathbf{x}_{b 1}, \mathbf{x}_{b 2}\right)=R_{A-1, B-2}\left(\mathbf{x} ; \mathbf{x}_{a 1}, \mathbf{x}_{a 2}, \mathbf{x}_{b 1}, \mathbf{x}_{b 2}\right) \\
& \int_{\chi_{a} \in\left\{\mathbf{x}_{A}\right\}} \int_{\chi_{b} \in\left\{\mathbf{x}_{B}\right\}} d^{2} \chi_{a} \quad d^{2} \chi_{b} C_{A, B}^{\delta}(\mathbf{s}) \Psi_{A}\left(\chi_{a}\right) \Psi_{B}\left(\chi_{b}\right)
\end{aligned}
$$

If I take the limit as $\left\{\mathbf{x}_{a}\right\} \rightarrow \mathbf{X}_{A}$ and $\left\{\mathbf{x}_{b}\right\} \rightarrow \mathbf{X}_{B}$, i.e. take the limiting case of smaller and smaller detectors,

$$
P(\mathbf{x}, \mathbf{s}) \rightarrow c \delta(s-x \cos \phi-y \sin \phi)
$$




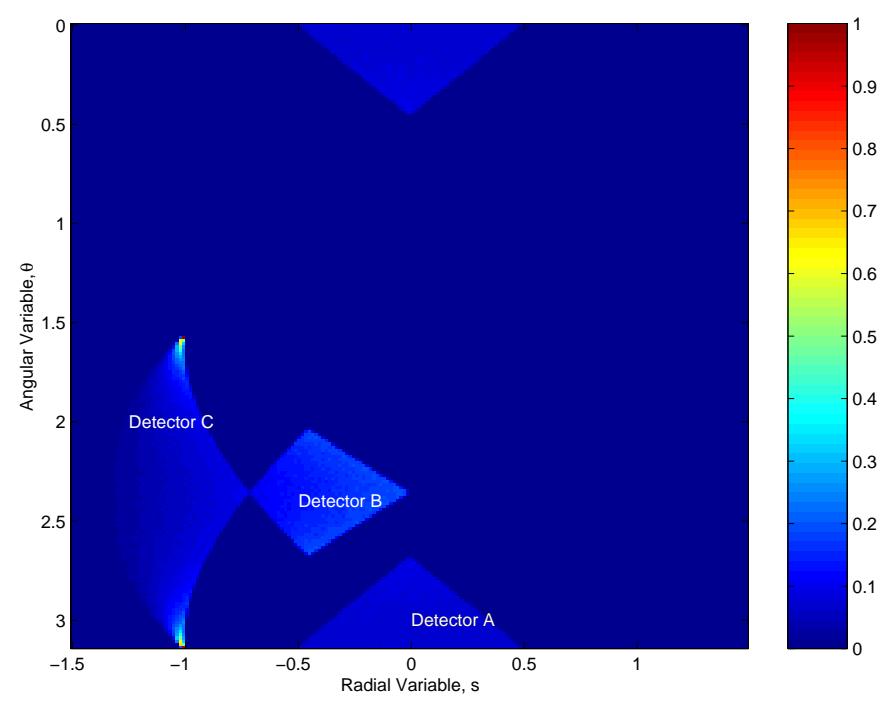

Figure 2.6: Examples of Chord Distributions. These are chord distributions of detectors pairs in Figs. 2.4 and 2.5 given that the probability of hitting each point on both line segments are all equal.

where $\mathbf{s}$ is defined by the points $\mathbf{X}_{A}$ and $\mathbf{X}_{B}$ for some constant $c$. The limiting case of the Chord Distribution is given by Eqn. 2.21. On the other hand, the limiting case of the Response Function can be determined by observing that its integral perpendicular to the line connecting the detectors is independent of its distance from either detector. Fig. 2.7 A shows the sum of the pixel values across the columns of image in Fig. 2.4 (Response Function A). Note that it is a constant. Fig. 2.7 B shows the sum of the pixel values along the diagonals of the Response Function B in Fig. 2.5 as the detector pair is taken to the limit $\mathbf{x}_{A} \rightarrow\{x=0.0, y=-1.0\}$ and $\mathbf{x}_{B} \rightarrow\{x=1.0, y=0.0\}$. The curves are the normalized integrals as the detectors are shortened in length by 0.2 . Thus, the Response Function tends to a constant value along the line defined by sh and within the confines of the detector pair.

The functional

$$
\Phi[f(\mathbf{x})]=\int_{D} d^{2} x \delta(s-\xi \cdot \mathbf{x}) f(\mathbf{x})
$$



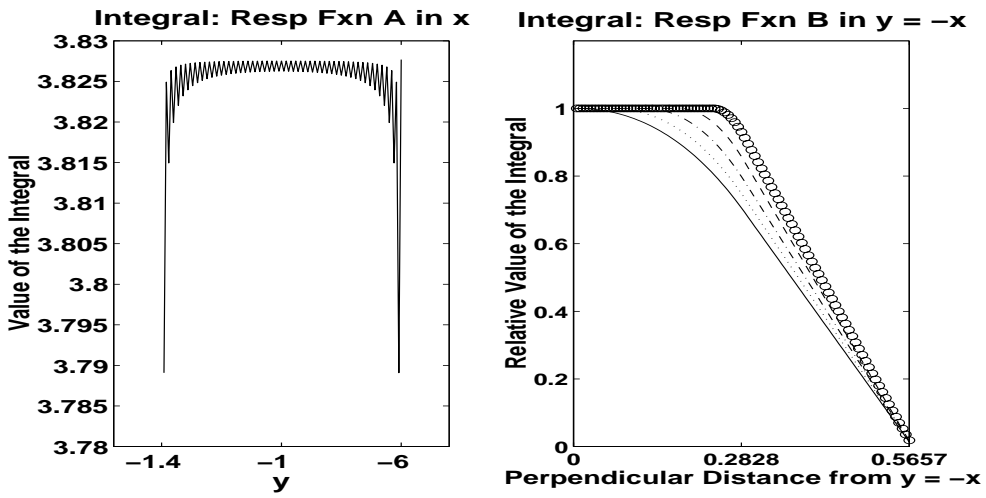

Figure 2.7: Integral of Response Function.

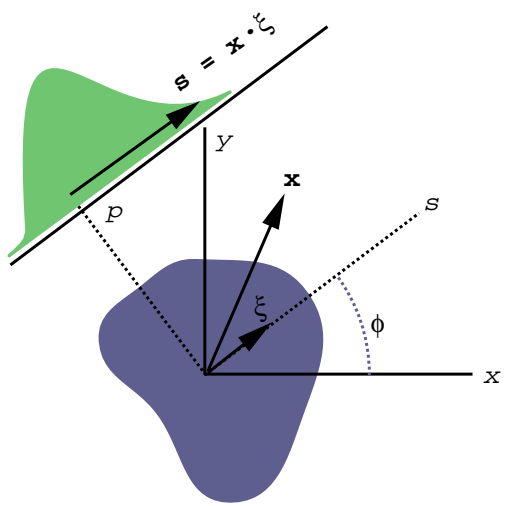

Figure 2.8: Coordinate System Used for Radon Transform. 
with $\xi=(\cos \phi, \sin \phi)$ is the called the Radon Transform, $\mathcal{R}$. It is thus named because of the pioneering work of Johann Radon [25] in inverting it. $\mathcal{R}$ in 2D maps the function $f$ on to its line integrals, i.e. it calculates the projections of $f$. Fig. 2.8 shows the coordinate system used by the Radon Transform. Therefore in the limit of infinitely small detectors, PET data is the Radon Transform of the radionuclide concentration in the subject being imaged. All that needs to be done is to invert the transform in order to determine the concentration map of the radionuclides.

The connection between PET and the Radon Transform is an extension of the transform's use in X-ray Computed Tomography (CT). In this imaging modality, the intensity of a beam of x-ray is measured after traversing the subject being scanned. Along the source-detector line $L$, the quantity

$$
\frac{I}{I_{o}}=\exp \left(-\int_{L} \mu(\mathbf{x}) d s\right)
$$

is measured. This model also assumes infinitely small detectors. The exponent on the right hand side is the line integral of the attenuation map. It is clear that the $\log$ of both sides of the equation yields the Radon transform on the right-hand side with the attenuation map of the object, $\mu(\mathbf{x})$, serving as the function $f$ in Eqn. 2.24.

\subsection{Radon Transform and Its Inverse}

\subsubsection{Examples of Radon Transform}

Fig. 2.9 shows three sample objects whose Radon transforms are in Fig. 2.10. The Radon variables $s$ and $\phi$ are plotted as rectilinear variables instead of a polar coordinate pair. The sets on which they are defined are discussed in $\S 2.2 .2$. In this coordinate system, the Radon transform of the $\delta$-function $\delta\left(\mathbf{x}-\mathbf{x}_{o}\right)$ is the sine curve $\delta\left(s-x_{o} \cos \phi-y_{o} \sin \phi\right)$ (Fig. 2.10 A). Note that this is the " $x$ - projection" of the vector $\mathbf{x}_{\mathbf{o}}$ onto the axes which are rotated by $\phi$ relative to the $x y$ axes. Because this projection has the locus of a sine curve, PET data are referred to as sinograms. Fig. 2.10 B is the Radon transform of a disk centered at the origin with $r=0.2$. It 

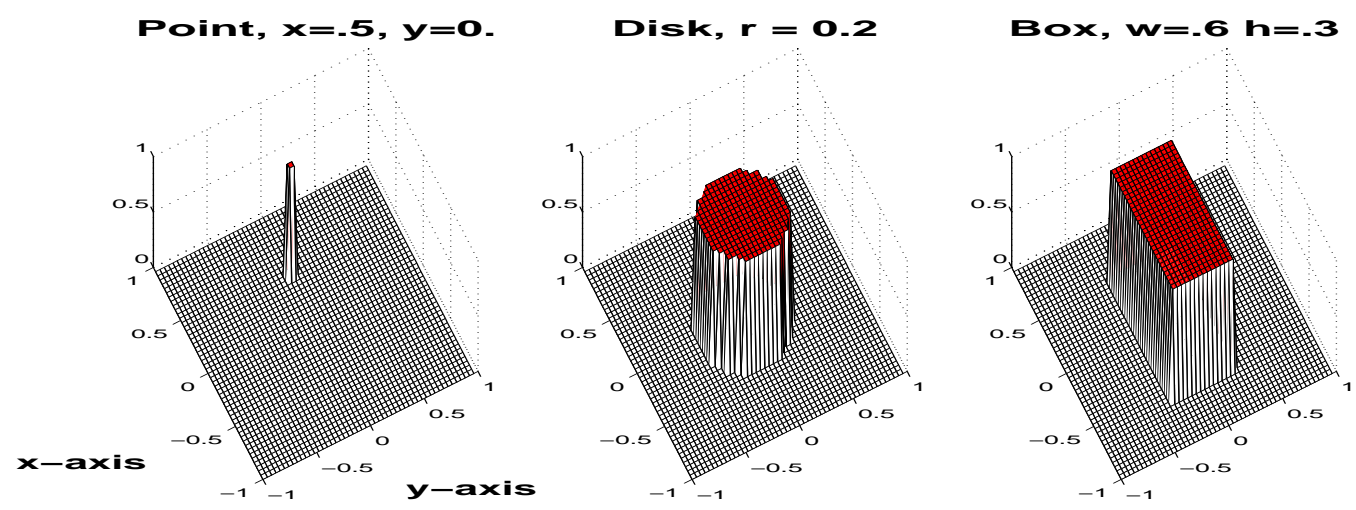

Figure 2.9: Sample Objects.

is independent of the projection angle because the disk is symmetric under rotation. Fig. 2.10 C is the transform of a box. Rattey and Lindgren [22] noted that considering $s$ and $\phi$ as a polar-coordinate pair leads to the incorrect assertion that $\mathcal{R} f(s=0)$ is independent of $\phi$. It is clear from the projection of the box that $\mathcal{R} f(s=0)$ is in fact dependent on $\phi$. The PEM camera produces a sinogram of this form if its FOV is flooded with constant radioactivity.

\subsubsection{Properties of Radon Transform}

Several properties of the Radon transform are essential to its inversion. Among them are a) Linearity b) Homogeneity and Symmetry c) and its relation to the Fourier Transform. I discuss these here but a more extensive treatment of the properties of the Radon transform appears in [23].

The linearity of the Radon Transform is easily seen by the following. Let $f$ and $g$ be two functions and $c_{1}, c_{2}$ be constants. Then

$$
\begin{aligned}
\mathcal{R}\left[c_{1} f+c_{2} g\right] & =\int_{D}\left[c_{1} f(\mathbf{x})+c_{2} g(\mathbf{x})\right] \delta(s-\xi \cdot \mathbf{x}) d \mathbf{x} \\
& =c_{1} \int_{D} f(\mathbf{x}) \delta(s-\xi \cdot \mathbf{x}) d \mathbf{x}+c_{2} \int_{D} g(\mathbf{x}) \delta(s-\xi \cdot \mathbf{x}) d \mathbf{x}
\end{aligned}
$$




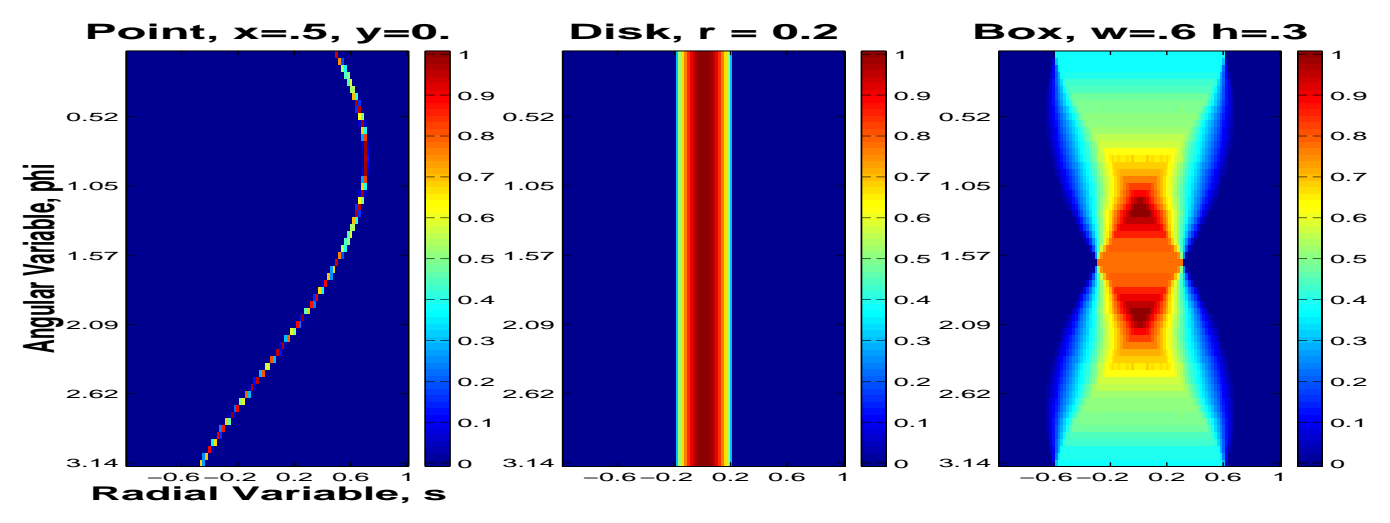

Figure 2.10: Radon Transforms of Sample Objects.

$$
=c_{1} \mathcal{R} f+c_{2} \mathcal{R} g
$$

A function $f$ is homogeneous of order (or degree) $a$ if for a given constant $t$

$$
f(t \mathbf{x})=t^{a} f(\mathbf{x})
$$

Observe that

$$
\begin{aligned}
\mathcal{R} f(a s, a \xi) & =\int_{D} f(\mathbf{x}) \delta(a s-a \xi \cdot \mathbf{x}) d \mathbf{x} \\
& =\left|a^{-1}\right| \int_{D} f(\mathbf{x}) \delta(s-\xi \cdot \mathbf{x}) d \mathbf{x}
\end{aligned}
$$

so that $\mathcal{R} f$ is homogeneous of order -1 . As a consequence of this, we have the symmetry property

$$
\mathcal{R} f(-s,-\xi)=\mathcal{R} f(s, \xi)
$$

which means that I can define $s$ and $\phi$ such that $s \in(-\infty, \infty)$ and $\phi \in[0, \pi)$.

The relation of the Radon Transform with the Fourier Transform is the basis for the Fourier Inversion Methods used in Reconstruction Tomography. This relation, first discussed by Bracewell [24], leads to the Central Slice Theorem which I define below. 
The Fourier transform $\mathcal{F}$ and its inverse $\mathcal{F}^{-1}$ are defined as (up to constants)

$$
\begin{aligned}
\mathcal{F} f & =\int_{\mathbf{R}^{2}} d^{2} x f(\mathbf{x}) e^{-i \mathbf{k} \cdot \mathbf{x}} \\
\mathcal{F}^{-1} F & =\int_{\mathbf{R}^{2}} d^{2} k F(\mathbf{k}) e^{i \mathbf{k} \cdot \mathbf{x}}
\end{aligned}
$$

Note that Eqn. 2.30 can be expressed as

$$
\mathcal{F} f(\mathbf{k})=\int_{\mathbf{R}} d t \int_{\mathbf{R}^{2}} d^{2} x f(\mathbf{x}) e^{-i t} \delta(t-\mathbf{k} \cdot \mathbf{x})
$$

In polar coordinates, $\mathbf{k}=k \xi$ where $k=\|\mathbf{k}\|$ and $\xi$ is the unit vector in the direction of $\mathbf{k}$. Hence, Eqn. 2.31 can be rewritten as

$$
\begin{aligned}
\mathcal{F} f(k \xi) & =\int_{\mathbf{R}} d t \int_{\mathbf{R}^{2}} d^{2} x f(\mathbf{x}) e^{-i t} \delta(t-k \xi \cdot \mathbf{x}) \\
& =\int_{\mathbf{R}} d\left(\frac{t}{k}\right) \int_{\mathbf{R}^{2}} d^{2} x f(\mathbf{x}) e^{-i t} \delta\left(\frac{t}{k}-\xi \cdot \mathbf{x}\right) \\
& =\int_{\mathbf{R}} d s e^{-i s k} \int_{\mathbf{R}^{2}} d^{2} x f(\mathbf{x}) \delta(s-\xi \cdot \mathbf{x}) \\
& =\mathcal{F}_{s} \mathcal{R} f
\end{aligned}
$$

where I have substituted $s=\frac{t}{k}$ in the second to the last step. The final step comes from the observation that the right-hand side of Eqn. 2.32 is the $s$ Fourier transform of the Radon transform. Thus the 2D Fourier transform of $f$ is the $1 \mathrm{D} s$ Fourier transform of its Radon transform.

The Central Slice Theorem is a direct consequence of Eqn. 2.32. It states that the Fourier components of the projection of a function in a direction perpendicular to $\xi$ and at a distance $s$ from the origin are equal to the Fourier components of the function along the direction $\xi$ through the origin in Fourier space. Given $\xi$ constant, note that the left-hand-side of Eqn. 2.32 are the Fourier components of $f$ along a line going through the origin in the direction of $\xi$. Also the right-hand-side are the Fourier components of the $\xi$ projection of $f$ a distance $s$ away from the origin. Fig. 2.11 shows the Central Slice Theorem graphically. The contours on the Fourier transform of the object is an attempt to indicate that the Fourier transform of a function with compact support has infinite support in Fourier space. 


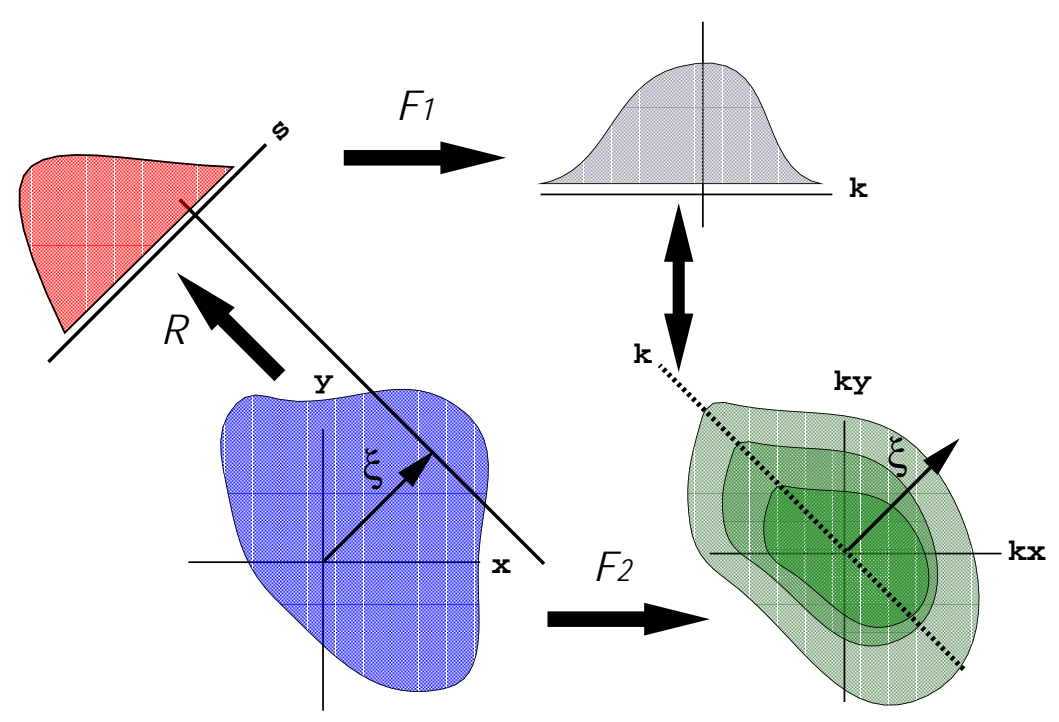

Figure 2.11: Central Slice Theorem in 2D.

\subsubsection{Inversion of Radon Transform}

I have shown that in the limit of small detectors, PET data is the Radon Transform of the radionuclide concentration map. I can thus determine the distribution of radionuclides in the subject being imaged by the inversion of the transform. The original 2D inversion of the Radon Transform was done by Johann Radon. He showed that continuous functions which have compact support $D$ have a unique $\mathcal{R} f$ determined by its projections along all lines $L$ intersecting $D$ (Eqn. 2.1). While I have considered 2D cases in our previous discussions, I present here an $n$-dimensional inversion of the Radon Transform in anticipation of the following chapter.

I begin with the Fourier inversion formula:

$$
f(\mathbf{x})=\int_{\mathbf{R}^{n}} d \mathbf{k} e^{i \mathbf{k} \cdot \mathbf{x}} F(\mathbf{k}) .
$$

Expressing Eqn. 2.34 in spherical coordinates

$$
f(\mathbf{x})=\int_{R}|k|^{n-1} d k \int_{\frac{\Omega}{2}} d \boldsymbol{\Omega}_{n} e^{i k \xi \cdot \mathbf{x}} F(k \xi)
$$


Note that I use the entire real axis for the integration of $k . d \Omega_{n}$ is the n-dimensional solid angle element ${ }^{3}$ while $\frac{\Omega}{2}$ denotes half the solid angle in n-dimensions. In $\mathbf{R}^{2}$, $\frac{\Omega}{2}$ is the half plane $\{\phi: \phi \in[0, \pi)\}$. In $\mathbf{R}^{3}$, while it is customary to use the top hemisphere $\frac{\Omega}{2}=\left\{\phi, \theta: \phi \in[0,2 \pi), \theta \in\left[0, \frac{\pi}{2}\right)\right\}$, I will use the lateral hemisphere $\frac{\Omega}{2}=\{\phi, \theta: \phi \in[0, \pi), \theta \in[0, \pi)\}$. I substitute the Central Slice Theorem (Eqn. 2.32) for the last term of the integral to get

$$
f(\mathbf{x})=\int_{\frac{\Omega}{2}} d \boldsymbol{\Omega}_{n} \int_{R} d k e^{i k \xi \cdot \mathbf{x}}|k|^{n-1} \mathcal{F}_{s} \mathcal{R} f(k) .
$$

The inversion diverges depending on whether $n$ is even or odd. When $n$ is odd, the absolute value sign is unnecessary. Recall that

$$
\mathcal{F}_{s}\left[\frac{d^{n}}{d s^{n}} h(s)\right](k)=i^{n} k^{n} \mathcal{F}[h](k)
$$

so that the inner integral in Eqn. 2.36 can be written as

$$
f(\mathbf{x})=i^{1-n} \int_{\frac{\Omega}{2}} d \boldsymbol{\Omega}_{n} \mathcal{F}_{k}^{-1}\left[\mathcal{F}_{s}\left[\frac{d^{n-1}}{d s^{n-1}} \mathcal{R} f(s)\right](k)\right](\mathbf{x} \cdot \xi) ; n \text { odd }
$$

I have suppressed the $\Omega$ dependence for brevity. However, I have explicitly expressed the integrand as a function of the variable $\mathbf{x} \cdot \xi$ which is critical in the completion of the inversion. The product $\mathbf{x} \cdot \xi$ is the dual variable of $k$ in the inverse Fourier transform. Note that both Fourier transforms are in $\mathbf{R}$.

Define the Backprojection operator $\mathcal{B}$ as

$$
\mathcal{B} g(\mathbf{x})=\int_{\frac{\Omega}{2}} g(\mathbf{x} \cdot \xi, \boldsymbol{\Omega}) d \mathbf{\Omega}_{n}
$$

where $g$ on the right-hand side is expressed in spherical coordinates and $\xi$ is the direction specified by $\phi$. Eqn. 2.38 can then written as

$$
f(\mathbf{x})=i^{1-n} \mathcal{B F}_{k}^{-1} \mathcal{F}_{s} \mathcal{D}_{s}^{n-1} \mathcal{R} f ; n \text { odd }
$$

where $\mathcal{D}$ is the derivative operator.

\footnotetext{
${ }^{3}$ Recall that $d \Omega_{2}=d \phi$ while $d \Omega_{3}=\sin \theta d \theta d \phi$.
} 
When $n$ is even, the absolute value sign remains and the less elegant formula is

$$
f=i^{1-n} \mathcal{B F}_{k}^{-1}|k|^{n-1} \mathcal{F}_{s} \mathcal{R} f ; n \text { even }
$$

Analytic reconstruction algorithms are implementations of this inversion formula. Because of the close relation of this equation with the Fourier transform, these algorithms are classified as Fourier Methods of Image Reconstruction.

Natterer [26] gives a detailed discussion of this inversion. He unifies the inversion in even and odd dimensions by using the Riesz Potential $\mathcal{I}$ defined as

$$
\mathcal{F}\left[\mathcal{I}^{\alpha} f\right](\mathbf{k})=|k|^{-\alpha} \mathcal{F}[f](\mathbf{k})
$$

He notes that the inner integral in Eqn. 2.36 can be expressed as a Riesz Potential. His inversion leads to the explicit use of the Hilbert Transform $\mathcal{H}$ which appears in the inversion formula only when the dimension $n$ is even. This has consequences for the inversion of the Radon transform in the following sense. The Hilbert Transform $\mathcal{H}$ is defined as

$$
\mathcal{H} f(s)=\frac{1}{\pi} \int_{\mathbf{R}} d t \frac{f(t)}{s-t} .
$$

Observe that this is the convolution of the function $f(s)$ with the function $s^{-1}$. This convolution leads to the non-locality of the inversion in that the determination of $f$ at a point $\mathbf{x}$ requires values of $\mathcal{R} f$ that does not involve $\mathbf{x}$. This means that in $2 \mathrm{D}$ reconstruction where $\mathcal{H}$ appears, one needs the projections through all the points in the support of $f$. However, since $\mathcal{H}$ does not appear in the $3 \mathrm{D}$ inversion, one does not need all the projections of $f$. Indeed, one can reconstruct a 3D object by stacking reconstructed $2 \mathrm{D}$ planes which are independent of each other.

\subsubsection{Backprojection and Filtering}

Natterer [26] noted that $\mathcal{R}$ and $\mathcal{B}$ form a dual pair in the sense that while $\mathcal{R}$ integrates over all points in a hyperplane of $\mathbf{R}^{n}$ (lines in $2 \mathrm{D}$ ), $\mathcal{B}$ integrates over all hyperplanes through a point. We now limit our discussion to $\mathbf{R}^{2}$ again. Fig 2.12(B) 

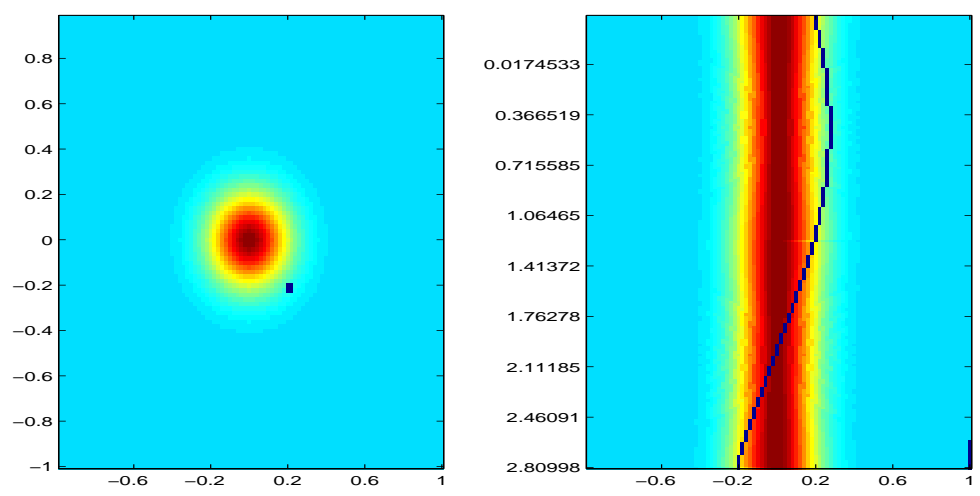

Figure 2.12: $r \phi$ as Rectilinear Variables. The Gaussian distribution centered at the origin in $x y$ (Left), its color indicating its height on the $z$ axis, appears as a tunnel-like structure that is independent of $\phi$ in $r \phi$ space (Right). A point maps to a sinusoid as shown in the plots. The ordinate in the right plot is the linear variable, $r$, while the abscissa is the polar-angular variable $\phi$.

plots the Gaussian distribution in Fig 2.12(A) using $r \phi$ treated as rectilinear variables instead of a polar-coordinate pair. Note that for a fixed $\left(x_{o}, y_{o}\right), r=\mathbf{x} \cdot \xi=x_{o} \cos \phi+$ $y_{o} \sin \phi$ follows a sine curve. Consider the point $(-0.2,0.2)$ as indicated in Fig 2.12 (A) and the corresponding curve as drawn in Fig 2.12 (B). Note that each point in $r \phi$ defines a line in $x y(\S 2.1 .3)$. This observation is in fact the basis for the Hough Transform developed by Hough in detecting straight lines in images [27]. Thus, Eqn. 2.39 integrates over all the lines through $\left(x_{o}, y_{o}\right)$ as $\phi$ takes on values from 0 to $\pi$.

The inversion in $\mathbf{R}^{2}$ reads:

$$
f=\mathcal{B} \mathcal{F}_{k}^{-1}|k| \mathcal{F}_{s} \mathcal{R} f
$$

The quantity that is backprojected is the Radon transform after its Fourier transform is multiplied by the function $|k|$. This is in fact filtering of the projection data with the Ramp Filter. The reason for this filtering becomes clear when we backproject the Radon transform alone. Fig. 2.13 is the backprojection of 1, 2, 4 and all of the 

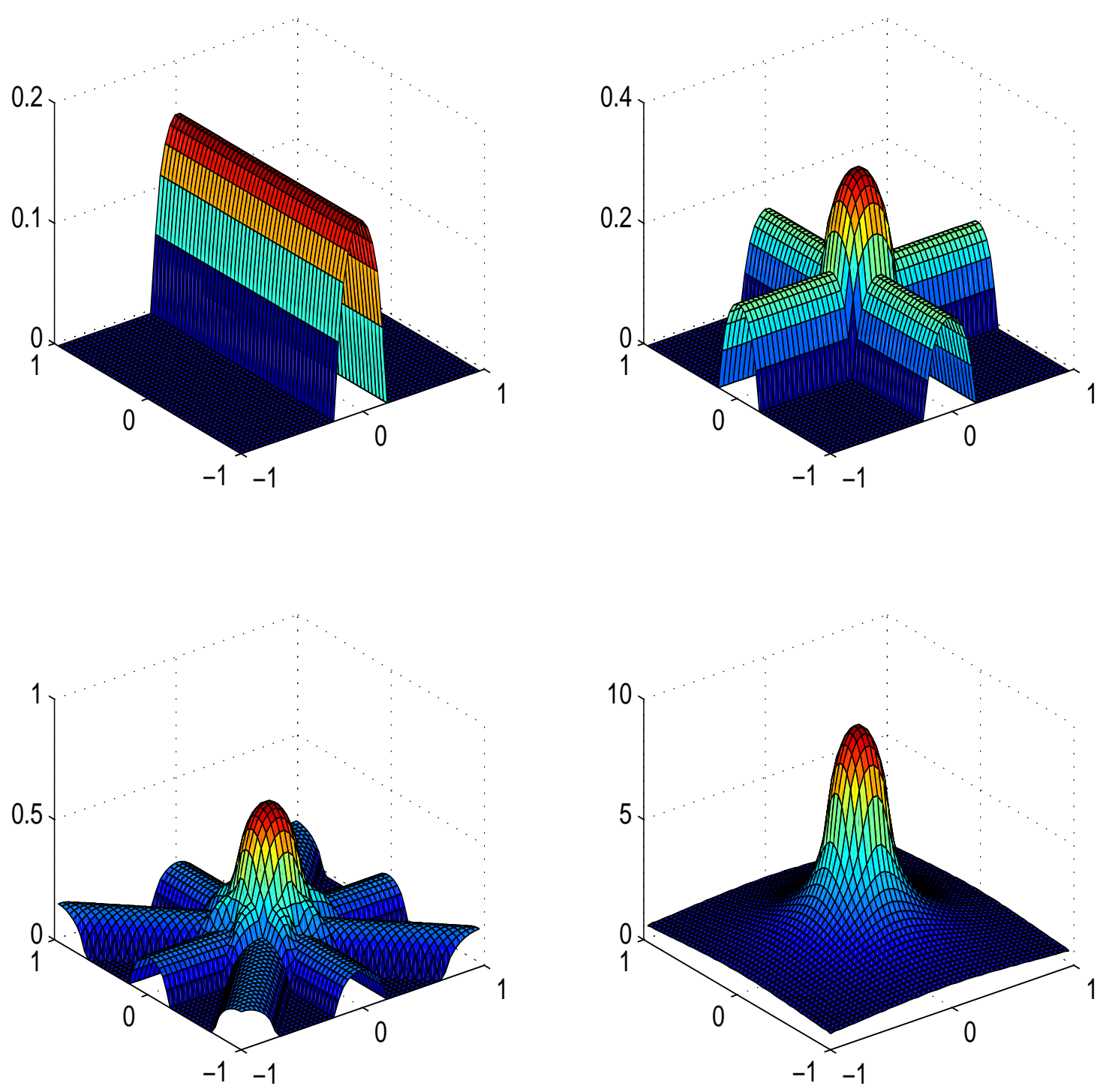

Figure 2.13: Backprojection of 1, 2, 4, and $\infty$ projections. 
projections of the sinogram in Fig. 2.10(B). Note that instead of the disk, Backprojection alone gives a blurred image of the object. In $\mathbf{R}^{2}$ this blurring is the result of the convolution of the object with $\frac{1}{r}$ [23]. Note that $\mathcal{F} \frac{1}{r}=|k|$. Since a convolution in coordinate space is a multiplication in Fourier space, the filtering of the sinogram by $|k|$ removes this blurring from the image.

In principle, all the essence of reconstruction tomography is contained in the discussions of the preceding section. However, we have made many assumptions in deriving the Radon transform model and its inversion. The main assumption is that our detectors are infinitely small. This gives us the complete Radon transform of the object in the FOV. Another assumption is that photons are not scattered in the object. In addition, photons are assumed not to penetrate into nor to scatter in the crystals. In reality, crystals have finite size. Photons scatter in and are attenuated by the object being scanned, and penetrate and scatter in the crystals. We will address these issues in the succeeding sections. In particular, we will address the case where the assumption of infinitely small detectors fails.

\subsection{Iterative Reconstruction Methods}

Another class of reconstruction techniques is the Iterative Reconstruction method. This method attempts to exploit the statistical nature of the data acquisition process in PET. This is accomplished by assuming a model for the data acquisition process, subjecting an initial guess at the radionuclide distribution to this model, comparing the resulting expected data with the measured data, and updating the guess until the expected data compares well with the measured data.

\subsubsection{Discretization of the Problem}

The issue of non-vanishing detector dimensions is at the heart of the statistical methods of reconstruction. Because there are only a finite number of detector pairs in a camera, as opposed to infinite if the detectors were indeed infinitely small, 
PET cameras can only acquire a finite number of measurements. In fact, the parameters of the sinogram $S(\mathbf{s})$ in Eqn. 2.1, instead of being continuous variables, take on only a finite number of values $s_{j}$ and $\phi_{k}$ where $0<j<N_{s}-1$ and $0<k<N_{\phi}-1$ such that $S(s) \rightarrow S\left(s_{i}, \phi_{k}\right)=S_{j k}$. Alternatively we can specify one index $i$ such that $S=S_{i}$ where $0<i<N_{s} N_{\phi}-1$. Thus, the system matrix $P$ in Eqn. 2.1 is a continuous to discrete functional

$$
S_{i}=\int_{D} d^{2} x P_{i}(\mathbf{x}) \lambda(\mathbf{x})
$$

In order to make the problem more tractable, the object is parametrized using basis functions as

$$
\lambda(\mathbf{x})=\sum_{j=0}^{N_{p}-1} \lambda_{j} b_{j}(\mathbf{x})
$$

where $N_{p}$ is the number of basis functions used. Eqn. 2.1 then becomes

$$
\begin{aligned}
S_{i} & =\int_{D} d^{2} x P_{i}(\mathbf{x}) \sum_{j=0}^{N_{p}-1} \lambda_{j} b_{j}(\mathbf{x}) \\
& =\sum_{j=0}^{N_{p}-1} \lambda_{j} \int_{D} d^{2} x P_{i}(\mathbf{x}) b_{j}(\mathbf{x}) \\
& =\sum_{j=0}^{N_{p}-1} \lambda_{j} P_{i j}
\end{aligned}
$$

The last equation can be written as the matrix equation $\mathbf{S}=\mathbf{P} \lambda$. The basis functions need to be chosen so that the object is well represented and the values $P_{i j}$ are easy to compute. Although by no means the only basis function used in image reconstruction, the most common choice of basis function is the Pixel Basis. It uses the Pulse Function $\Pi\left(\mathbf{x} ; \mathbf{x}_{o}, \mathbf{w}\right)$ defined in Eqn. 2.3. In this case, $N_{p}$ is the number of pixels in the image and $P_{i j}$ is interpreted as the probability that an annihilation event that occurred in pixel $j$ is detected in the sinogram index $i$.

Thus the inverse of the system matrix $\mathbf{P}$ gives the solution to the reconstruction problem. Unfortunately, there are issues that make the inversion of $\mathbf{P}$ 
difficult at best. Firstly, the dimensions involved in PET reconstruction are very large. For typical 2D images of $1,024 \times 1,024=1,048,576$ pixels, the number of bins can approach the same order of magnitude and so we have the problem of inverting a $1 \mathrm{Mx} 1 \mathrm{M}$ matrix. This problem becomes prohibitively large in 3D imaging, where not only does the number of voxels ${ }^{4}$ increase by the cube, but the dimensionality of the projection space increases by the fourth power. Secondly, $\mathbf{P}$ is ill conditioned, i.e. small changes in $\lambda$ may result in large changes in $\mathbf{S}$. The condition number of a matrix is defined as the ratio of its largest and the smallest singular values. Thirdly, the $\mathbf{P}$ is not square and thus may have a null space. This means that there maybe objects in the FOV that project to the zero vector. Fourthly, because of the statistical nature of the data acquisition process, the data in the sinogram $\mathbf{S}$ is finite and thus has noise. Each sinogram bin is an independent random variable with counting statistics, i.e. Poisson statistics, and thus the data is inconsistent.

\subsubsection{Objective Functions and Statistical Models}

In essence, Iterative Reconstruction is finding an iterative solution to Eqn. 2.47. This is done in the absence of an algebraic solution to the problem (e.g. Minimum Norm Least Squares [28]) where the solution is obtained in one step. An iterative solution maybe expressed as the solution that minimizes an objective function.

One such objective function is the Maximum Likelihood [29]. The likelihood $L$ may be defined as the probability of realizing the data $\mathbf{d}$ given a model with a set of parameters $\boldsymbol{\Phi}[30]$, i.e $p(\mathbf{d} \mid \mathbf{\Phi})=L(\mathbf{\Phi} ; \mathbf{d})$.

Another familiar objective function is the $\chi^{2}$ which we define as

$$
\chi^{2}(\lambda)=\sum_{i} \frac{\left(\sum_{j} P_{i j} \lambda_{j}-S_{i}\right)^{2}}{\sigma_{S_{i}}^{2}}
$$

where $S_{i}$ are the measured sinograms and $\sigma_{s_{i}}$ are the corresponding uncertainties. Note that a sum over the pixel index $j$ is a projection operation while a sum over

\footnotetext{
${ }^{4}$ Voxels, or volume elements, are 3D analogs of pixels
} 
the sinogram index $i$ is a backprojection operation. It is evident then that the backprojection operation is the matrix transpose of the projection operation, i.e. $B=P^{T}$. In fact, redefining $\mathbf{S}$ and $\mathbf{P}$ such that

$$
\begin{aligned}
\mathcal{S}_{i} & =S_{i} / \sigma_{s_{i}} \\
\mathcal{P}_{i \bullet} & =\mathbf{P}_{i \bullet} / \sigma_{s_{i}}
\end{aligned}
$$

Eqn. 2.48 written in matrix notation is

$$
\chi^{2}(\lambda)=\lambda^{T} \mathcal{P}^{T} \mathcal{P} \lambda-2 \mathcal{S}^{T} \mathcal{P} \lambda+\mathcal{S}^{T} \mathcal{S}
$$

This formulation may be interpreted as a Maximum Likelihood solution to the reconstruction problem with the assumption that the measured data $\mathbf{S}$ has a Gaussian distribution centered at $\mathbf{S}$ with uncertainties $\sigma_{i}=\sqrt{S_{i}}$. This is appropriate if the data are precorrected for randoms and scatter, and attenuation and detector efficiency. Fessler [31] observed that the common procedure of subtracting accidental coincidence events from the sinogram [32] shifts the statistics of PET data such that a Gaussian model is the more appropriate distribution rather than Poisson. While it is true that the Poisson distribution maybe approximated by the Gaussian distribution for large means as predicted by the Central Limit Theorem [33], he shows that even for small true rates and $10 \%$ randoms, a Gaussian model is more appropriate.

\subsubsection{Pros and Cons of Iterative Reconstruction}

There are benefits of posing the reconstruction problem as a statistical problem. Among them are that constraints on the problem can be incorporated into the reconstruction in a consistent manner. An example of these constraints is the physical constraint of positivity. Since the image represents the radiotracer density, all pixels in the reconstructed image must have a non-negative value. $A$ priori information may also be incorporated into the reconstruction procedure as well. This information may be from other imaging modalities such as MRI and CT. 


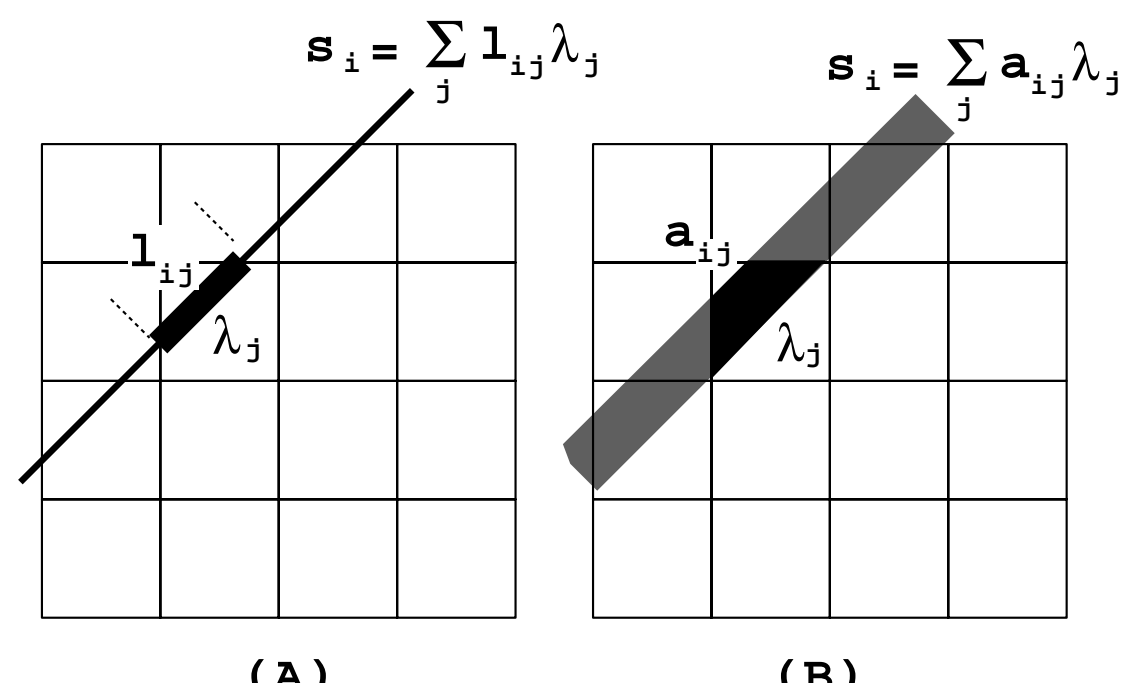

(A)

(B)

Figure 2.14: Models used to calculate System Matrix elements. (A) is the line length model and (B) is the strip area model.

An example of a priori information used in iterative reconstruction today is the object boundary which can be determined by subjecting the object to a CT scan before the PET scan. Statistical models may also be used in the reconstruction such that the statistical significance of individual LOR's are appropriately used. Filtered Backprojection treats all the LOR's equally.

There are, however, disadvantages to using iterative techniques. An accurate model of the data acquisition process is needed. The comparison between the expected and measured data is only as good as how well the expected data can be modeled. There are two common approximate methods for calculating the elements of the matrix $\mathbf{P}$, both of which are detailed in $R E C L B L$ [34]. The first one calculates the length of the chord that lies within a pixel boundary. The contribution of this pixel to the sinogram bin is the length multiplied by the pixel value. The second is to calculate the area of intersection between the pixel and the strip defined by the boundaries of the sinogram bin. Fig. 2.14 shows these two methods. Note that 
these methods do not include the solid angle factor in the calculation of the system matrix elements. More recently, Huesman et al. [35], in attempting fully 3D list mode iterative reconstruction with the PEM camera, uses the line length method but draws several lines through the voxel (the 3D equivalent of a pixel) and includes the solid angle factor. List mode reconstruction performs image reconstruction on an event-by-event basis instead of the histogrammed sinogram data.

Another disadvantage of using iterative reconstruction techniques is in the software implementation. It is more complex than the filtered backprojection. In addition, the computation time involved can be an order of magnitude longer than filtered backprojection. There are, however, efforts to reduce the computation time with more efficient algorithms. It is unlikely that computing power alone will solve all of the computational difficulties of image reconstruction [36].

Finally, the nonlinearity of the problem makes statistical characterization of images in terms of noise difficult [37]. Often approximations are used to make the problem tractable, as for example, the approximate expressions for image bias and covariance in generalized-EM algorithms derived by Barret et al. [38] and Wang et al. [39]. These approximations are made out to be functions of the iteration number. 


\section{Chapter 3}

\section{The Camera}

The Positron Emission Mammography (PEM) camera is an optimization of conventional PET cameras. It is intended to be used to image human breasts as an application of nuclear medicine techniques in breast cancer detection and management. In this chapter, I describe the geometry and the components of the camera, the data acquisition system, the data flow and initial processing before they are stored, the construction of the modules that make up the camera, and finally the construction of the camera itself. I also describe the process for measuring the DOI information.

\subsection{Detector Module}

Central to the success of the PEM camera is a PET detector module that addresses the problem of crystal penetration. Crystal penetration leads to degradation of camera resolution away from the center of circular tomographs, a problem called Radial Elongation. The severity of the problem increases with increasing angle of incidence between the $511 \mathrm{keV}$ photons and the crystal detectors. Conventional PET cameras are typically circular with a FOV covering half its radius, i.e. the maximum angle of incidence between a $511 \mathrm{keV}$ photon and a crystal is $\frac{\pi}{6}$. The 
rectangular geometry of the camera together with extension of the FOV to include nearly the entire area enclosed by the detector modules allow angles of incidence far greater than those encountered in conventional PET machines, exacerbating the problem of Radial Elongation.

\subsubsection{Camera Geometry}

The PEM camera is rectangular in shape for a several reasons. One of the motivations for developing the PEM camera is to achieve greater sensitivity over fullbody PET machines currently in use. This is accomplished by bringing the modules in as close as possible to the breast. Because the modules are parallelpipeds of fixed size, packing them into any shape will result in some fraction of the detector volume not covered by detector material. These gaps wil result in regions in the FOV with either low detection efficiency or unsampled. A rectangular packing scheme minimizes these gaps in the detector volume. The individual modules are ganged together into four independent banks. The corners of the rectangle remain a problem but they can be remedied by overlapping adjacent banks. The minimum overlap between banks is designed to be be two crystal widths $(6 \mathrm{~mm})$.

Another reason for the rectangular shape is so that the camera is able to accomodate breasts of varying sizes. The long edges of the camera may be moved. This design also has the benefit of allowing the soft compression of the breast during the scan to prevent motion which would lead to artifacts in the reconstructed image. In the case of PET, these artifacts are manifested by further blurring and loss of resolution [40].

Incidentally, bringing the modules as close to the subject as possible has the added benefit of minimizing the effect of acollinearity. As previously discussed, the two $511 \mathrm{keV}$ photons are acollinear because the lab frame is not the rest frame of the positron-electron system. The acollinearity results in an additional blurring of the image that is proportional to the effective diameter of the camera and which 
can be modeled as a Gaussian blur with a $\mathrm{FWHM}^{1}$ of $0.028 \mathrm{~mm} /(\mathrm{cm}$ diameter) [41]. The closer the modules are to the breast, the smaller an effect acollinearity becomes.

Thus, while the geometry of the camera worsens radial elongation, it nontheless needs to be rectangular. Of course if the depth of interaction were measured, the geometry of the camera is immaterial.

\subsubsection{Previous attempts at correcting Radial Elongation}

In the past, there have been generally two ways of dealing with crystal penetration and radial elongation. One strategy is to determine the blurring function throughout the field of view and deconvolve it out of the image. Huesman et al. [42] performs this type of deconvolution on the data before it is reconstructed. They accomplish this deconvolution by modeling the data acquisition in PET as a linear system

$$
\langle\mathbf{s}\rangle=\mathbf{P} \lambda
$$

where $\mathbf{s}$ is the vector representation of the measured data, the \langle\rangle indicating its expectation, $\mathbf{P}$ is a matrix representing the data acquisition process and $\lambda$ as the radionuclide concentration to be determined. The problem of image reconstruction is discussed in Chapter 2. In particular, $\mathbf{s}$ is called the sinogram while $\mathbf{P}$ is called the system matrix which is defined in $\S 2.1 .1$. The method discussed has the flavor of Iterative Reconstruction ( $\$ 2.3)$ in the begining. However, the deconvolution of the radial blurring is performed on the data before it is used to reconstruct the object. Once the data is corrected for the blurring, the object is reconstructed using filtered backprojection [43] with a ramp filter.

The blurring is modeled as a matrix operator $\mathbf{G}$ that is applied to the data as

$$
\mathbf{s}^{*}=\mathbf{G}\langle\mathbf{s}\rangle
$$

\footnotetext{
${ }^{1} \mathrm{FWHM}$ is the point at which a Gaussian distribution is half its maximum value. It corresponts fo $2.35 \sigma$.
} 
where $\mathbf{s}^{*}$ is taken to be the sinogram that is measured by the camera. In order to get a reconstructed image that does not have radial blurring, the inverse of $\mathbf{G}$ is applied to the measured sinogram before the reconstruction is performed. $\mathbf{G}$ is calculated by determining all the possible chords that a crystal-face to crystal-face chord maps to when an event at the crystal face is allowed to penetrate into the bulk of the crystal. The penetration is modeled according to an exponential distribution.

The results indicate that this procedure amplifies the noise in the reconstructed image with only a modest improvement in radial resolution. In addition, this result was obtained using the infinite count limit, i.e. the number of events in the data set is large enough that statistical fluctuation is a small effect. Huesman predicted that in finite data sets, the statistical correlations in the correction matrix will cause the pixels of the image to have a high degree of correlated noise.

The other strategy is to deal with the problem in hardware. However, the most obvious ways of treating the problem of crystal penetration all lead to a reduction in the sensitivity of the tomograph. Decreasing the crystal length increases the likelihood that the crystal of interaction is the first crystal penetrated by the photon; but this means that the stopping power is reduced and thus sensitivity is compromised. The diameter of the tomograph may be increased so that the incidence angle of the photon is minimized, but this means that the solid angle covered by the detector, and thus the sensitivity, is decreased. Finally an absorbing metal may be placed between crystals to prevent photons from penetrating into adjacent crystals. Keller et al. [44] studied the use of tungten septa of a few millimeters. Their Monte Carlo simulations indicated that an improvement of $15 \%$ in resolution is obtainable at a loss of $200 \%$ in sensitivity.

A number of alternative module designs that measure DOI are based on the phoswhich design, i.e. stacking short crystals that have different properties into a single "crystal." DOI is measured by determining in which short crystal the interaction occured. One design was conceived and built by Carrier, et al. [45]. Their design used a $\mathrm{Bi}_{4} \mathrm{Ge}_{3} \mathrm{O}_{12}(\mathrm{BGO})-\mathrm{Gd}_{2} \mathrm{SiO}_{5}: \mathrm{Ce}(\mathrm{GSO})$ phoswhich, with crystals of the 
same length staggered (and rotated) to minimize degenerate chords. This configuration of crystals, accomodates the four (4) sample per detector width requirement for the Nyquist condition [46] to be satisfied. In fact, Derenzo [47] observed that if the modules of a stationary PET ring are rotated so that the long axes of the crystals do not intersect at the center of the tomograph, DOI information can be used to provide continuous linear sampling. The module used avalanche photodiodes for readout. They observed a 15\% light loss in the BGO-GSO boundary. The reconstructed images indicated that although the resolution was more uniform throughout the entire FOV, the resolution at the center of the tomograph degraded.

A more recent example of the phoswhich design is the three-crystal module built by Seidel et al. [48]. The module is composed of three layers of crystal scintillators, each $4 \mathrm{~cm}$ in length. The crystals are $\mathrm{Lu}_{2} \mathrm{SiO}_{5}$ : $\mathrm{Ce}(\mathrm{LSO})$ [49], GSO, BGO. In this case, provided that there are no degenerate chords, there is a potential 9-fold increase in the sampling rate of the tomograph. Coupled to the module is a PMT that integrates the light output of the crystal of interaction. Because the decay rates of the crystals are $40 \mathrm{~ns}, 60 \mathrm{~ns}$, and $300 \mathrm{~ns}$ respectively, the ratio of the total integrated signal to the signal integrated over a short window close to the trigger will be greatest for an interaction that occurs in the LSO and least for an interaction that occurs in the BGO. This method is called Delayed Charge Integration. This phoswhich module has an average successful identification fraction of approximately $83 \%$.

Other modules include one which used the temperature dependence of the light output of scintillation crystals [13], one which had a position sensitive photodiode along the length of the crystal [47], and one which had a strip of absorbing black band around a portion of the crystal to differentiate the front versus the back of the crystal [21]. The black strip in the last example was placed in a location such that the probability that the photon will interact in the front section of the crystal is equal to the probability that it will interact in the back section of the crystal. This addresses the nonuniform count rate between the two segments which, if not 


\begin{tabular}{lccc}
\hline \hline & $\mathrm{NaI}(\mathrm{Tl})$ & BGO & LSO \\
\hline Density $\rho\left(\mathrm{g} / \mathrm{cm}^{3}\right)$ & 3.67 & 7.13 & 7.4 \\
\hline Effective atomic no. & 51 & 75 & 66 \\
\hline Radiation Length $\lambda(\mathrm{cm})$ & 2.56 & 1.12 & 1.14 \\
\hline Index of refraction $n$ & 1.85 & 2.15 & 1.82 \\
\hline Relative Emission Intensity & 100 & 15 & 77 \\
\hline Peak Wavelength $\lambda_{P}(\mathrm{~nm})$ & 410 & 480 & 420 \\
\hline Decay Constant $\tau(\mathrm{ns})$ & 230 & 300 & 40 \\
\hline \hline
\end{tabular}

Table 3.1: Scintillator Properties of NaI, BGO, and LSO.

considered, leads to the increase in the noise level of the image [50].

\subsubsection{Lutetium Orthosilicate}

In conventional PET modules, scintillation light produced by an interacting $511 \mathrm{keV}$ photon is collected by one photodetector, most often a photomultiplier tube. However, the PEM modules measure DOI by comparing the scintillation light collected from one end of a crystal with that from the other end. Because the scintillation light is shared between two photodectors, the amount of light impinging on each detector is lower than if there were only one photodector attached to one end. It is therefore critical that the scintillator used for the PEM modules has high light yield.

The scintillator of choise is LSO. First classified as a scintillator by Melcher et al [49], LSO is a monoclinic C crystal with unit cell parameters $a=14.254 \AA, b=$ $10.241 \AA, c=6.641 \AA, \gamma=122.20^{\circ}$ [51]. The crystals are grown as $\mathrm{Lu}_{2(1-x)} \mathrm{Ce}_{2 x}\left(\mathrm{SiO}_{4}\right) \mathrm{O}$ where the Ce atoms serve as activiation sites for the scintillation process. Undoped LSO does not scintillate. The properties of LSO are summarized in Table 3.1 together with the properties of $\mathrm{BGO}$ and $\mathrm{NaI}(\mathrm{Tl})$ for comparison.

LSO has scintillation and mechanical properties that make it desirable for our application. Among them are its high density and short radiation length, both of 


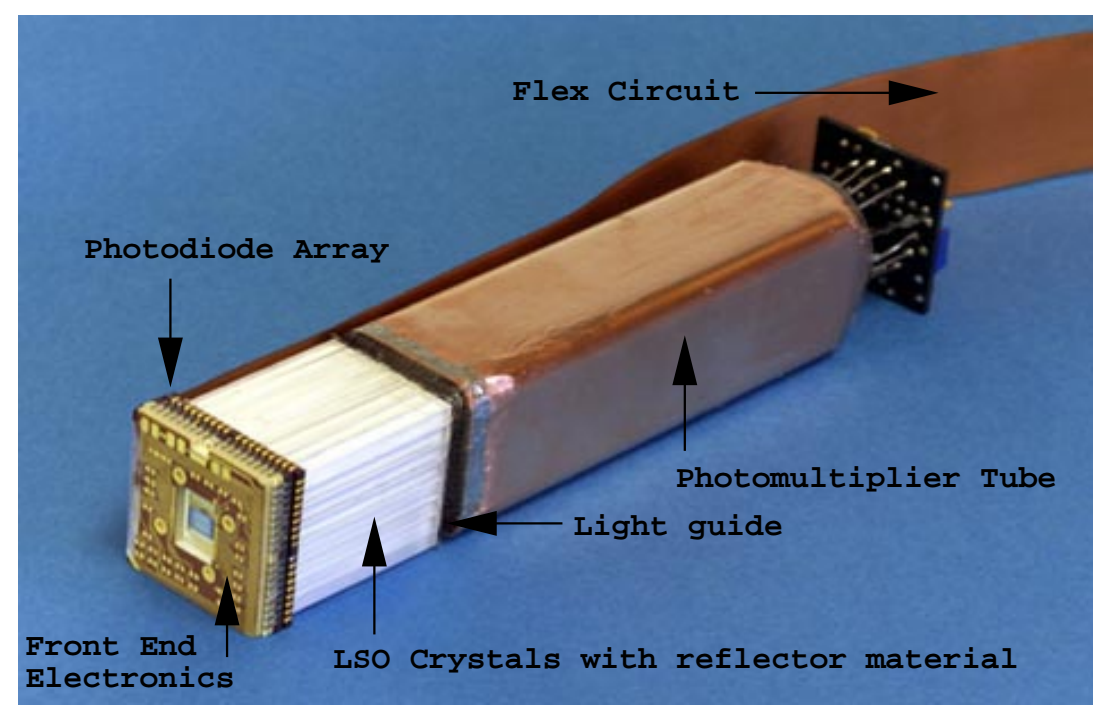

Figure 3.1: The PEM Module. The main componenets of the module are the LSO crystals, the front end custom IC, the photodiode array, the PMT and the flex circuit.

which are comparable to BGO. Its high effective $Z$ ensures a high photoelectric cross section which is proportional to $Z^{4<n<5}$ [52]. Its index of refraction is similar to that of glass so that reflection is minimized between the crystal-PMT interface. Its peak emission wavelength is $420 \mathrm{~nm}$, which is a good match for bialkali photomutliplier tubes that we use, and it is transparent to its own scintillation light. It has an order of magnitude faster decay constant than BGO, and in fact has an even faster decay component of 12 ns which has a relative fraction of $35 \%$. It is rugged, and unlike $\mathrm{NaI}$, it is not hygroscopic.

The crystals used for the PEM camera have the dimensions $3 \times 3 \times 30 \mathrm{~mm}^{3}$. With 42 modules, each an $8 \times 8$ array of crystals, the PEM camera requires 0.72581 of LSO. We acquired the LSO crystals from CTI-PET Systems, located in Knoxville, TN, USA. 


\subsubsection{Photodetectors}

Fig. 3.1 shows a completed PEM module. We use two devices to collect the signal from the LSO. The signal from the PMT serves as the trigger and initiates the readout in the data acquisition system. The signal from the pixelized photodiode (PD) array identifies the crystal of interaction. The ratio of the signal from the PD and the sum of the signals from the PD and the PMT determines the depth at which the $511 \mathrm{keV}$ photon interacted within the crystal.

The PMT used for the camera is a Hamamatsu R2497 PMT. It is a 10-stage linear PMT that operates at $1.5 \mathrm{kV}$ with a typical gain of $2.5 \times 10^{6}$ and maximum dark current of $250 \mathrm{nA}$. Its spectral response ranges from $300 \mathrm{~nm}$ to $600 \mathrm{~nm}$ with a peak at $420 \mathrm{~nm}$. It uses a bialkali metarial for the photocathode and its window is made of borosilicate glass. Its typical response time is approximately $2.4 \mathrm{~ns}$. Its dimensions are $2.5 \times 2.5 \mathrm{~cm}^{2}$ by $10 \mathrm{~cm}$.

The PD is a PIN type device consisting of an $8 \times 8$ array of photosensitive elements. The device is backside illuminated, i.e. the pixels have a common photosensitive $n$-layer and a pixellated $p$-layer. Each pixel is $3 \times 3 \mathrm{~mm}^{2}$. The device has a quantum efficiency of $\geq 70 \%$ in the $380-600 \mathrm{~nm}$ range. Because of the low number of scintillation photons from the LSO crystal, and the fact that these photons are shared between the PD and the PMT, it is important for the PD to have as little noise as possible. Typical signals in the PD are expected to be between 1000 and $3000 \mathrm{e}^{-}$. The $\mathrm{PD}$ is operated with $50 \mathrm{~V}$ at room temperature $\left(25^{\circ} \mathrm{C}\right)$ with dark current less than $200 \mathrm{pA}$. Each pixel has a terminal capacitance less than $5.0 \mathrm{pF}$ and a series resistance less than $50 \Omega$. The signals from the PD is processed by a custom IC and are delivered to the downstream electronics by a Kapton flex circuit. 


\subsection{Data Acquisition System}

The data acquisition system for the PEM camera can be divided into three major components: a) the front-end electronics b) the initial data processing boards and c) the coincidence processing. The front-end electronics is composed primarily of the PET64, a custom IC that processes the signal from the PD. The initial data processing boards are circuit boards modeled after the electronics of the CTI-PET systems which are used for the High Resolution Research Tomograph (HRRT). The boards were originally designed to take in four PMT signals as input and perform Anger Logic type calculations to determine the crystal of interaction. They are therefore modified to accomodate the signals from the PD and as well as to generate control signals for the PD. The coincidence processing boards are taken directly from CTI as "off the shelf" components.

\subsubsection{Front End Electronics}

The central piece in the front end electronics is the PET64, a custom IC designed and built by the Lawrence Berkeley National Laboratory Engineering Division. The PET64 has two main components: a 64 channel charge sensitive amplifier and the circuitry that determines which of the 64 channels is associated with the crystal that is hit by the $511 \mathrm{keV}$ photon, the Winner Take All (WTA). Controls for the charge sensitive amplifier and the output of the WTA are brought in from and out to the Analog Subsection respectively by a flex circuit board made of Kapton.

The custom IC has 64 channels with an area of about $4.0 \times 5.0 \mathrm{~mm}^{2}$. The rise time, fall time and bias currents of each channel are all adjustable parameters via an external current source. The gain of the amplifier can be varied by a factor of 4 in 64 steps by an external control determining the feedback capacitance of the amplifier. The PEM modules are anticipated to operate the amplifiers at the highest gain and rise and fall times of approximately $1 \mu$ s and $3 \mu$ s respectively. Fig. 3.2 shows a schematic of one channel of the amplifier. 


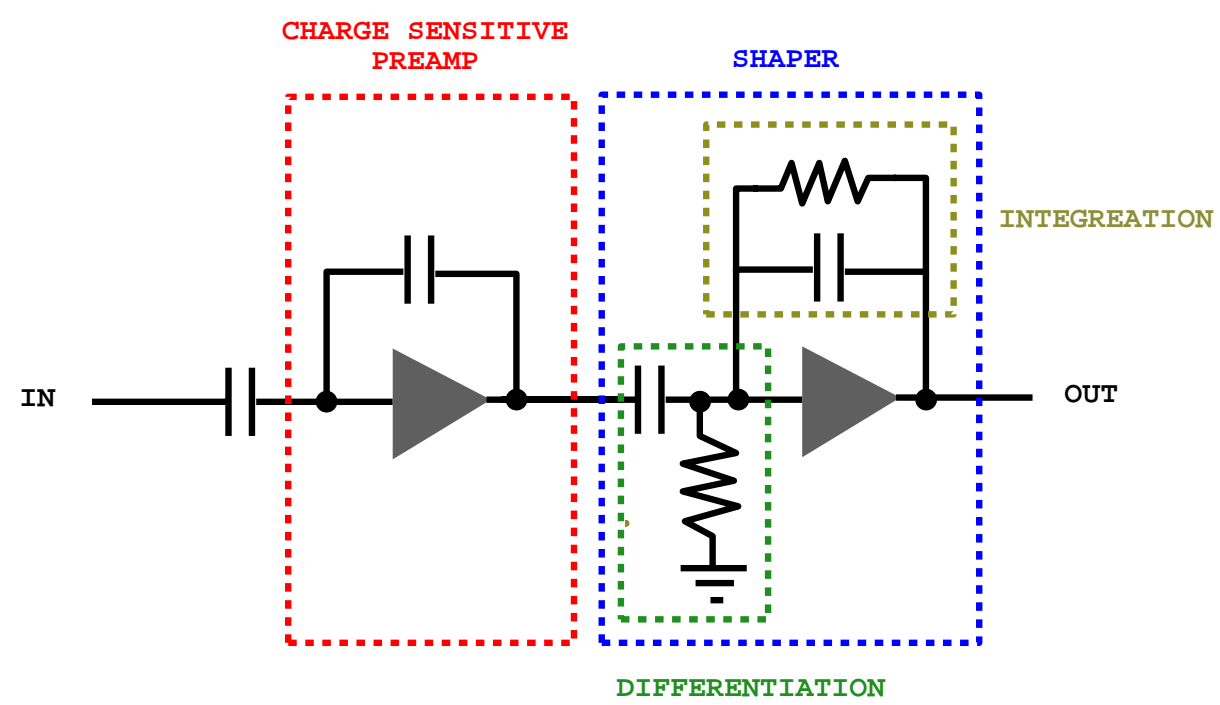

Figure 3.2: Schematic of one channel of charge sensitive amplifier

The small signal in the PD of $1000-3000 \mathrm{e}^{-}$coupled with a typical noise of $470 \mathrm{e}^{-}$FWHM together with Compton scatter complicates the event topology so that a simple threshold scheme will often give indeterminate results, either with zero or greater than one pixel above threshold. In order to determine the crystal of interaction correctly and rapidly $(<100 \mathrm{~ns})$, the WTA circuit [53] is used for the PEM front end electronics. The WTA is composed of 64 amplifiers, each with only two transistors, and all supplied by a common limited current source such that only one of the amplifiers can be turned on. The chosen channel, the one with the highest input signal, is encoded and is used to select a multiplexer input which is the output of the amplifier circuit.

\subsubsection{Data Flow Control}

The signals from the PMT and the PD are acquired by the Analog Subsection Board (ASB), shown in schematic in Fig. 3.3. A PMT signal that is above the 
prescribed threshold in the Analog ASIC serves as the event trigger. The trigger is sent to a programmable logic array that performs the timing and initial data processing. The system clock of the Analog Subsection has a period of 32 ns, so in order to get the 2 ns timing resolution required by the coincidence board downstream, the trigger is sent through a delay line that resolves the time stamp into sixteen 2 ns divisions. The trigger and the verniered time constitute the time stamp of the event. The signals to sample and hold the front end amplifier outputs are then sent to initiate the output sequence of the PD. Approximately $256 \mathrm{~ns}$ after the trigger, the Altera FPGA strobes the flash ADC and captures the PMT digitized signal. Approximately $1 \mu \mathrm{s}$ after the trigger, the Altera FPGA strobes the other ADC and captures the PD digitized signal of the winning channel in the photodiode array. The address of the winning channel is also latched by the Altera FPGA at this time.

Once the PD and PMT signals have been digitized and latched, crystaldependent corrections are applied to them so that they can be added and their ratios taken. The correction is done through lookup tables stored in RAM chips on board. The RAM chips take the PD address and PD and PMT signals as inputs. The correction scales the input values with the appropriate multiplier.

The corrected PMT and PD values are then used to determine whether or not to accept the event, and if so, to calcualte the DOI. The signals are added to get the total energy of the event. This energy is used to qualify the event whether it is consistent with a $511 \mathrm{keV}$ event or not. The threshold for the energy is set to approximately $350 \mathrm{keV}$. The qualification is performed in another lookup table stored in another RAM. The inputs of the RAM are the corrected PMT and PD values. They serve as addresses of the RAM which contains essentially a flag, determining whether the event is good. If the event is in the acceptance window, the DOI is calculated, again using a lookup table. The same corrected PD and PMT signals are used as addresses in the RAM where the ratios $\frac{P D}{P D+P M T}$ have been previously stored.

The entire event word, comprised of the time stamp, the PD address, the 


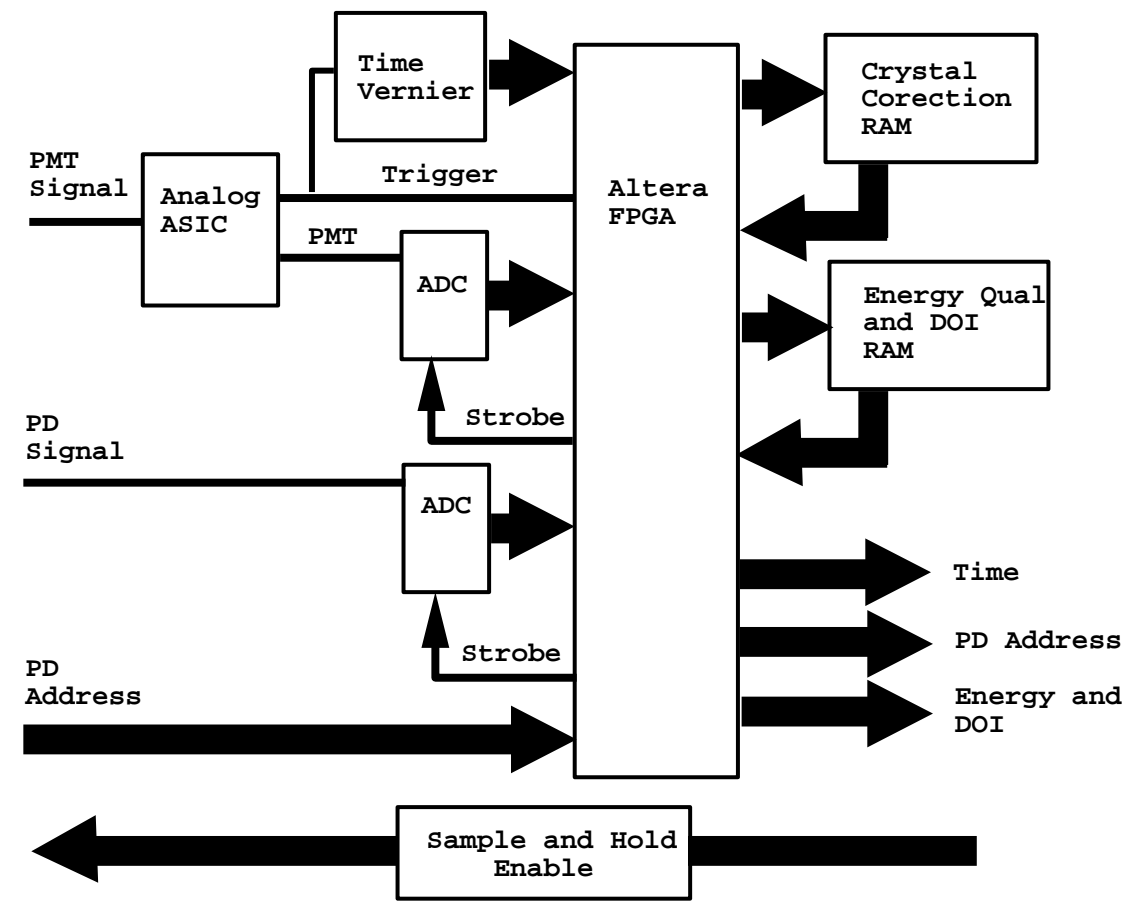

Figure 3.3: Data flow and processing in the Analog Subsection 


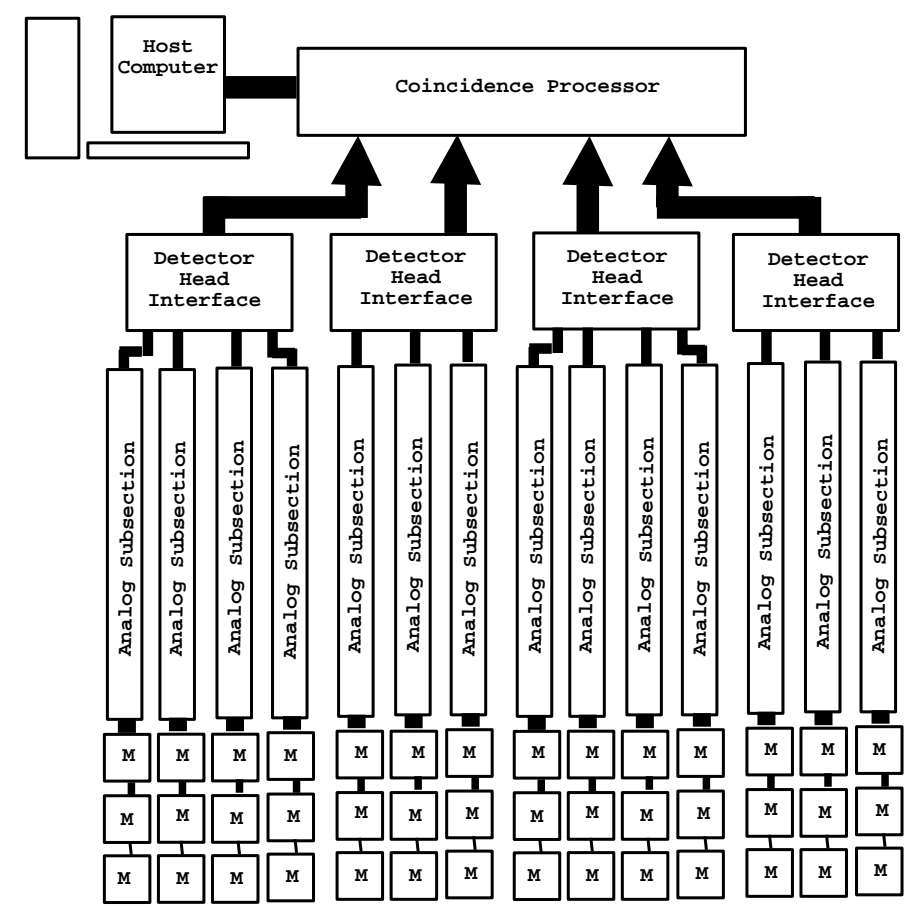

Figure 3.4: Electronics organization for the PEM data acquisition system.

energy qualification bit and DOI bits are then clocked out of the Altera FPGA board. The entire event processing takes approximately $1 \mu \mathrm{s}$, during which the entire detector block is paralyzed. Each detector module is therefore able to handle a 1 $\mathrm{MHz}$ single-event rate.

Fig. 3.4 shows the data flow after the event is pushed out of the ASB. Each ASB receives data from three modules in parallel and delivers the information to the Detector Head Interface (DHI). The DHI serves as a data funnel for up to five ASB. The DHI can receive up to fifteen singles data in parallel but puts out only four. Each detector bank has its own DHI so there are a total of four DHIs for the PEM camera. The events are then transfered to the coincidence processor which determines if there are any hits from two unique DHIs within a 2 ns window. These events are tagged as 
coincidences and are written in list-mode to disk. The coincidence processor is able to handle coincidence rates of up to $4 \mathrm{MHz}$. The events may also be histogrammed into projection data immediately after being tagged as coincidences, but that will require rebinning in order to properly treat the DOI information. The possibility of using of CTI's rebinner board for the purposes of the PEM camera was being deliberated at the time of the writing of this dissertation.

\subsection{Construction and Calibration}

The surface of the LSO needs to be treated so that the number of scitillation photons detected at each of the photodetectors will have a depth dependence ${ }^{2}$. The treatment of the LSO crystals is described in detail by Huber et al. [54]. In order to reduce processing and handling of the crystals, they are chemically etched instead of mechanically polished. The crystals are etched in a pyrophosphoric acid bath at $200^{\circ} \mathrm{C}$ for approximately $5 \mathrm{~min}$. The crystals are then placed in a jig so that strips of reflector material can be placed on each of the four long sides. The entire crystal bulk is placed in an evacuated glass bell where the PD is glued on one end and a light guide-PMT structure on the other. The gluing is done in vacuum in order to minimize the formation of bubbles in the glue coupling the photodetectors to the LSO. Bubbles in the glue can adversely affect the light collection of the photodetectors. The frontend electronics are wire-bonded to the PD array and the entire module is placed in an parallelpiped aluminum can together with two other modules. The camera is then composed of these cans stacked vertically on their sides, four on each of the long banks of the camera and three on the short banks.

Initial measurements of the depth dependence of the signal were performed with a set-up described by Moses et al. [55] and shown in Fig. 3.5. However, when the camera is constructed, it will be impossible to access each individual crystal in

\footnotetext{
${ }^{2}$ Depth dependence is defined as the ratio of the signal observed when the crystal is excited at the end closest to and furthest from the detector.
} 


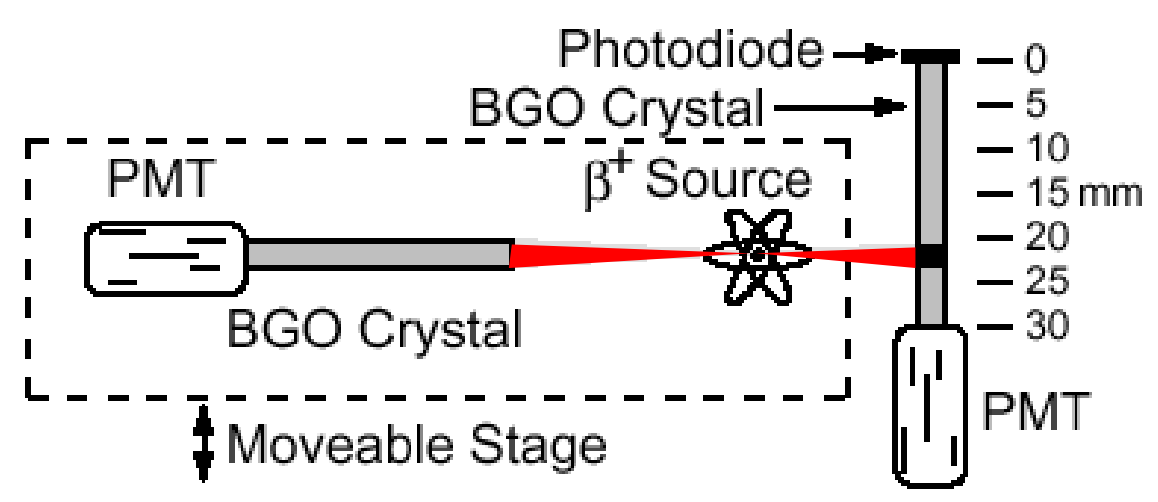

Figure 3.5: Setup for DOI Measurement (After Moses).

each module. In calibrating the modules there are two parameters that need to be determined: the gain ratio between the photodetectors and the depth dependence factor. The gain ratio is essential so that the signals from the PD and the PMT can be summed. Huber et al. [56] describes two methods of calibrating the detector in situ by shining $511 \mathrm{keV}$ photon from the end of the crystals instead of the side. The first method uses the fact that, because of the exponential nature of the interaction length, the most likely interaction point is that which is closest to the PD (recall that the $\mathrm{PD}$ end is closest to the breast). The signal distribution of both photodetectors will then have peaks at the energy bins associated with events in which the interaction point is at the $\mathrm{PD}$ end. The ratio of these energy bins should determine the depth dependence of the signal. The second method uses the fact that the total energy of the events is independent of the depth of interaction if the gain between the photodetectors were correct. Simulations indicate that both gain ratios and depth dependence can be reasonably determined such that the energy and DOI resolution are only minimally degraded. 


\section{Chapter 4}

\section{Reconstruction in 2D}

In order to study the sampling issues without the complications of 3D image reconstruction, I begin my investigation of reconstruction algorithms for the PEM camera by limiting the problem to $2 \mathrm{D}$. I do this with the intention of extending successful methods to a $3 \mathrm{D}$ reconstruction of objects within the camera. I use Monte Carlo simulation to generate annihilation events from which I build the sinogram data that I reconstruct.

\subsection{Simulation}

In order to study the algorithms for image reconstruction we are developing, we need to reconstruct images using them. It would be ideal if we have real data from which to reconstruct objects. However, since detector development and algorithm development are simultaneously occurring, it is necessary to generate Monte Carlo data with which we can form images.

\subsubsection{Phantom Simulation}

I begin by assuming a phantom in the FOV representing a continuous distribution of radiotracers. In this thesis, phantoms will generally be classified as point 
sources or extended phantoms. The Point Spread Functions (PSF), the reconstructed image of a point source, give a measure of the expected resolution of the camera and the reconstruction algorithm. I measure the image resolution by a Region of Interest (ROI) study. In the study concentric regions (usually circles or spheres) are drawn around an area in the FOV within which the total activity is calculated as a function of distance from the center of the reconstructed point source.

The PSF can also reveal gross non-local artifacts that can indicate problems with the reconstruction algorithm. Artifact formation can be seen as irregularities in the reconstructed point-source image. Non-local artifacts are usually streak artifacts that radiate out from the point source. As image reconstruction depends on small correlations between projections, mismatches in coordinate system can also appear in point source reconstructions.

Extended phantom reconstruction provides a measure of noise and overall image quality. Noise measurements are done with a similar ROI study. The mean value of a set of pixels is compared to their covariance and the result can be interpreted as the signal-to-noise ratio that maybe used to evaluate the image as reconstruction parameters are varied. Streak artifacts can also be exhibited in extended phantom images. Objects that appear in reconstructed extended phantoms but were not included in the simulation are indications of possible reconstruction errors or inappropriate reconstruction parameters.

A location in the FOV is randomly chosen according to the given phantom distribution. This is used as the location of the particular radiotracer currently being generated. As mentioned in $\S 1.2$, the ejected $e^{+}$, losing energy primarily through collisions [57], actually traverses a finite distance before annihilating with an $e^{-}$. I did not include this positron range in my simulation because ${ }^{18} F$, which has a $\beta$-decay end-point energy of $0.6350 \mathrm{MeV}$ [58], has a positron range of $\lambda_{e^{+}}=0.254$ $\mathrm{mm}$ [59]. The resolution of the camera will be dominated by the crystal size of 3.0 mm. As I shall show, the reconstructed PSF without positron range is of the order of $2.0 \mathrm{~mm}$. Thus, a $0.254 \mathrm{~mm}$ blurring from the positron range will not contribute 


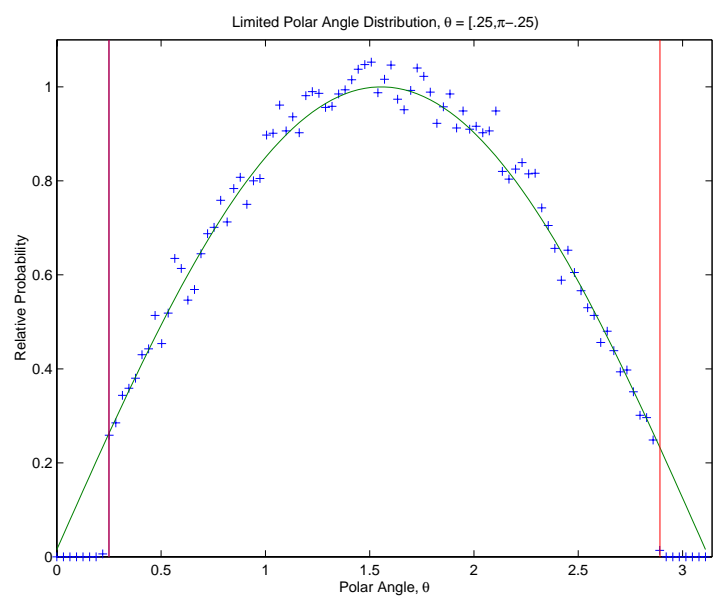

Figure 4.1: Example of generation of limited polar angle, $\theta$.

greatly when added in quadrature.

A random direction is then determined. The distribution of directions is taken to be flat in the solid angle $\sin \theta d \theta d \phi$ for a specified range of the polar angle. This restriction in the polar angle is taken into account when determining acceptances and sensitivities which are defined as the fraction of $511 \mathrm{keV}$ photons detected by the camera and the fraction that survives event selection respectively. Fig. 4.1 shows an example of the distribution of polar angles if the limits are defined as $\theta \in[.25, \pi-.25)$. The distribution for $\phi$ is uniform. The acollinearity with a $0.5^{\circ}$ FWHM between the annihilation photons can be toggled in the simulation.

\subsubsection{Photon Tracking}

The simulation allows for phantoms with non-zero attenuation, so I will discuss how I track the photons through the the FOV assuming there is an attenuating medium within it. While the results that I present in this dissertation do not contain scatter in the FOV, I nonetheless discuss scattering in the FOV for three reasons: a) I model scatter in the crystals b) concurrent work in list-mode iterative 
reconstruction for the PEM detector models scatter in the FOV c) future work which should include quantization with the PEM camera, will necessitate the modeling of scatter in the FOV. Scattering in the crystals of the detector modules follow the same procedures as scattering in the FOV, differing only by the attenuation constant and photofraction (LSO vs. scatter medium in the FOV).

I track both $511 \mathrm{keV}$ photons through the phantom, allowing them to interact via either Compton scattering or photoelectric effect. I calculate the energydependent cross sections of Compton scattering and photoelectric effect by using the equation

$$
\sigma^{C S, P E}(E)\left[\mathrm{cm}^{2}\right]=\frac{N_{A} 10^{-24}}{w} \sum_{i} n_{i} \sigma_{i}^{C S, P E}(E)
$$

where $N_{A}$ is Avogadro's number, $n_{i}$ is the number of the $i^{\text {th }}$ atom present in the molecule and $w$ is the molecular weight. For water, $n_{H}=2, n_{O}=1$ and $w_{H_{2} O}=18$ g. I calculate the photofraction by taking the ratio $P E=\frac{\sigma^{P E}(E)}{\sigma(E)}$, and the linear attenuation length by using $\mu(E)=\rho \sigma(E)$, where $\rho$ is the density of the material. The distance covered by the photon before interacting is drawn from an exponentially decaying distribution using $\mu$ as the decay length. I use the photofraction to determine the interaction type.

If the interaction is a Compton scatter, I perform the following procedure. I rotate the photon direction towards $\hat{z}$ so that the scattering angle can be easily determined. I draw the azimuthal scattering angle from the flat distribution $[0,2 \pi)$ , and the polar scattering angle according to the Klein-Nishina [60] formula

$$
\frac{d \sigma}{d \Omega}=Z r_{0}^{2}\left(\frac{1}{1+\varrho(1-\cos \theta)}\right)^{2}\left(\frac{1+\cos ^{2} \theta}{2}\right)\left(1+\frac{\varrho^{2}(1-\cos \theta)^{2}}{\left(1+\cos ^{2} \theta\right)[1+\varrho(1-\cos \theta)]}\right)
$$

with $\varrho=\frac{E}{m_{0} c^{2}}$. The energy of the scattered photon is given by

$$
E^{\prime}=\frac{E}{1+\varrho(1-\cos \theta)}
$$

according to the conservation of energy and momentum. I then rotate the scattering angles back to the original direction of propagation. I do not track the recoiled 

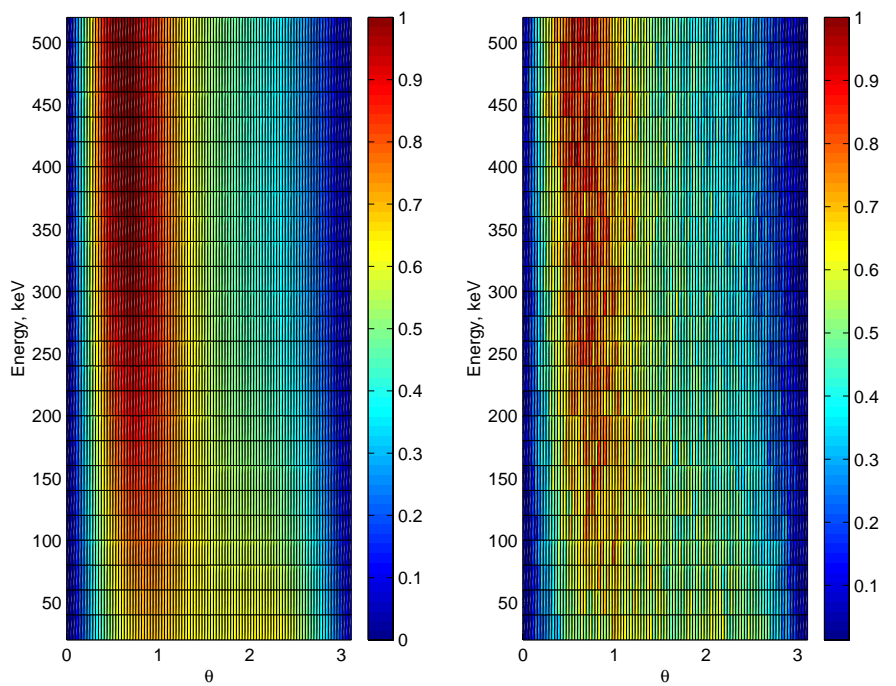

Figure 4.2: Histogram of generated scattering angle as a function of energy. The left plot is theoretical while the right plot is generated by the simulation.

electron which is assumed to deposit all its energy at the point of interaction in the phantom. The left plot of Fig. 4.2 shows the expected distribution of the scattering angle as a function of the energy of the photon while the right plot shows a histogram generated by the simulation.

I then track the photon to the detector face where it interacts with the LSO crystals in the same manner as discussed above. Fig. 4.3 shows the photofraction and the $\log$ of the attenuation of LSO that I used in the simulation. The data that I used to calculate the attenuation length of LSO are tabulated by Plechaty et al. [61]. I continue to track the photon until it interacts via photoelectric effect or leaves the detector volume. At each interaction, I assume that all the energy lost by the photon is converted into scintillation photons. I determine the number of scintillation photons $n_{\gamma}$ by multiplying the energy of the interaction with the conversion factor of LSO, taken to be 25,000 scintillation photons/MeV. I add statistical noise to the number of scintillation photons assuming a normal distribution with the variance 

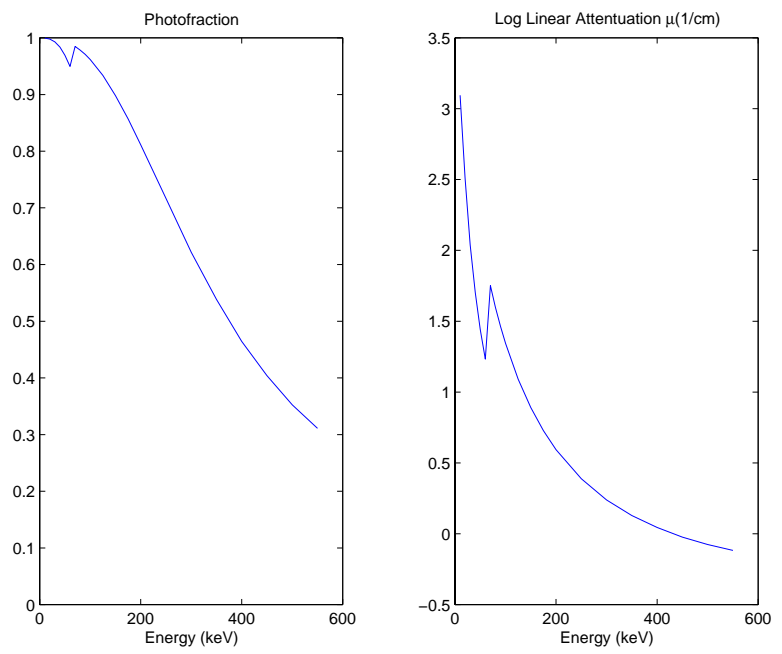

Figure 4.3: Photoelectric Fraction and Linear Attenuation Coefficient of LSO

equal to the number of photons.

The total number of scintillation photons is divided between the PD and the PMT such that $n_{\gamma}^{P D}+n_{\gamma}^{P M T}=n_{\gamma}$ where $n_{\gamma}^{P D}$ and $n_{\gamma}^{P M T}$ are linear functions of the depth of interaction $z$ :

$$
\begin{aligned}
n_{\gamma}^{P D}(z=0) & =\alpha n_{\gamma}^{P M T}(z=0) \\
n_{\gamma}^{P D}\left(z=L_{\text {crystal }}\right) & =n_{\gamma}^{P M T}\left(z=L_{\text {crystal }}\right) / \alpha
\end{aligned}
$$

for some constant $\alpha . z=0$ is an interaction at the photodiode end of the crystal while $z=L_{\text {crystal }}$ is an interaction at the photomultiplier end. Thus, we have

$$
\begin{aligned}
n_{\gamma}^{P D}(z) & =n_{\gamma} \frac{z+\alpha\left(L_{\text {crystal }}-z\right)}{(\alpha+1) L_{\text {crystal }}} \\
n_{\gamma}^{P M T}(z) & =n_{\gamma} \frac{\left(L_{\text {crystal }}-z\right)+\alpha z}{(\alpha+1) L_{\text {crystal }}}
\end{aligned}
$$

I use $\alpha=3$ and $L_{\text {crystal }}=3.0 \mathrm{~cm}$. The number of photons impinging on the photodetectors is then multiplied by the quantum efficiencies and gains of the photodetectors. 


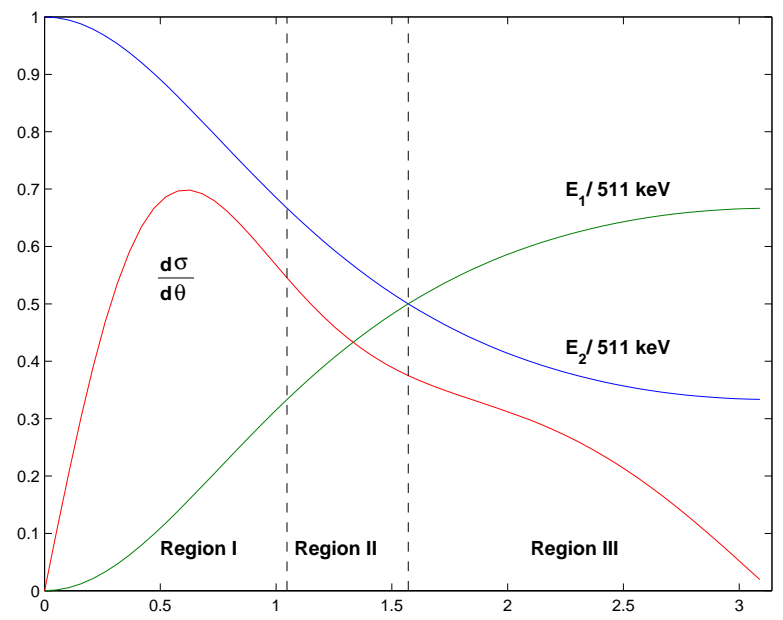

Figure 4.4: Compton Kinematics. The $x$ axis are in radians while the $y$ axis is relative probability.

Statistical and electronic noise are added (in quadrature) to the signal recorded for the photodetectors.

\subsubsection{Detector Compton Scatter}

Compton scatter in the detectors can lead to mispositioning of events. If a photon deposits energy in more than one crystal, the location of the first interaction must be identified in order to assign the event to the correct LOR. With crystal dimensions $0.3 \times 0.3 \times 3.0 \mathrm{~cm}^{3}$, the probability that a normally incident $511 \mathrm{keV}$ photon deposits energy in one, two, and more than two crystals is $70 \%, 24 \%$ and $6 \%$ respectively.

We studied algorithms to correctly identify crystals of first interaction in Compton scatter events [62]. All the algorithms attempted to exploit the fact that energy and momentum conservation require the energy of the scattered photon is given by Eqn. 4.3 with $\varrho=1$. Fig. 4.4 shows the kinematics of events with one 


\begin{tabular}{lcccc}
\hline \hline & Max Signal & Reject Mult & Sec Highest & Joint \\
\hline EFF $\tau=0 \mathrm{keV}, \mathrm{N} / \mathrm{S}=.4$ & $83 \%$ & $0 \%$ & $83 \%$ & $83 \%$ \\
\hline EFF $\tau=250 \mathrm{keV}, \mathrm{N} / \mathrm{S}=.4$ & $79 \%$ & $68 \%$ & $79 \%$ & $79 \%$ \\
\hline EFF $\tau=350 \mathrm{keV}, \mathrm{N} / \mathrm{S}=.4$ & $65 \%$ & $65 \%$ & $65 \%$ & $65 \%$ \\
\hline MIF $\tau=0 \mathrm{keV}, \mathrm{N} / \mathrm{S}=.4$ & $23 \%$ & $\mathrm{NA}$ & $\mathrm{NA}$ & $\mathrm{NA}$ \\
\hline MIF $\tau=250 \mathrm{keV}, \mathrm{N} / \mathrm{S}=.4$ & $21 \%$ & $19 \%$ & $28 \%$ & $23 \%$ \\
\hline MIF $\tau=350 \mathrm{keV}, \mathrm{N} / \mathrm{S}=.4$ & $15 \%$ & $14 \%$ & $14 \%$ & $\mathrm{NA}$ \\
\hline ROI $\tau=0 \mathrm{keV}, \mathrm{N} / \mathrm{S}=.5$ & & & & \\
$r$ at $90 \%$ Containment & $3.2 \mathrm{~mm}$ & $3.4 \mathrm{~mm}$ & $3.4 \mathrm{~mm}$ & $3.4 \mathrm{~mm}$ \\
\hline \hline
\end{tabular}

Table 4.1: Results of algorithm optimization for determining detector Compton scatter

Compton scatter. If $E_{1}$ is the energy deposited at the interaction point and $E_{2}$ the energy of the scattered photon (which is assumed to deposit all of its energy at another crystal), this relationship implies that there are three kinematic regions: I) Unambiguous forward scatter. When $\theta<\frac{\pi}{3}, E_{1}<E_{2}$ and there is no $\theta^{\prime}$ such that $E_{1}^{\prime}=E_{2}$ (and $E_{2}^{\prime}=E_{1}$ ). Therefore the crystal of interaction can be unambiguously determined from the energy alone. II) Ambiguous forward scatter. When $\frac{\pi}{3}<\theta<\frac{\pi}{2}$, $E_{1}<E_{2}$ but there is a $\theta^{\prime}$ such that $E_{1}^{\prime}=E_{2}\left(\right.$ and $\left.E_{2}^{\prime}=E_{1}\right) . \theta=\frac{\pi}{3}$ and $\theta^{\prime}=\pi$ is an example of such a pair. Therefore, the crystal of first interaction cannot be unambiguously determined from the energy values alone, as backwards scatter (i.e. events from Region III) produces events that yield identical energy deposits. III) Backwards scatter (always ambiguous). When $\theta>\frac{\pi}{3}$ there is always a $\theta^{\prime}$ such that $E_{1}^{\prime}=E_{2}$, (i.e. events from Region II).

Algorithm performance is evaluated with three figures of merit: a) the Singles Efficiency $(E F F)$, defined as the number of events an algorithm assigns a crystal of interaction (correctly or otherwise) divided by the total number of events generated (this includes inefficiencies caused by the photon traversing the detector without interacting), b) the Misidentification fraction $(M I F)$ defined as the number of misidentified events divided by the number of events in which an algorithm assigns 
a crystal of interaction, and c) a region of interest (ROI) analysis of the reconstructed image that plots the relative activity in circular regions of interest as a function of the radius of the region. The ideal algorithm has high EFF, low MIF and a steep rise in the ROI curve. For the purpose of this analysis, we use a line source near the center of a circular tomograph. This simplifies the ROI analysis where standard reconstruction packages for circular reconstruction with regular sampling may be used. As the results are dependent solely on the geometry and characteristics of the module instead of the whole scanner, applying them to the PEM camera is justified.

Four algorithms were evaluated: a) Maximum Signal. This algorithm selects the channel with the largest measured energy deposit as the crystal of first interaction, rejecting events where the maximum signal is below an energy threshold $(\tau[\mathrm{keV}])$; b) Reject Multiples Algorithm. This algorithm eliminates Compton interactions by rejecting events with two or more signals above an energy threshold; c) Second Highest Signal Algorithm. This algorithm selects the channel with the smaller signal when two signals are above the energy threshold. d) Joint algorithm. This algorithm is a hybrid algorithm, performing as the Maximum signal when the N/S ratio is low and as the Second highest algorithm when the N/S ratio is high. N/S ratio is the ratio of the electronic noise to the average signal generated by a $511 \mathrm{keV}$ photon. The performance of the Joint algorithm is accomplished by comparing the difference between the highest and the second highest signals, EDIFF, to a second energy threshold, $\delta_{E}$. The crystal with the second highest signal is chosen if EDIFF $<\delta_{E}$, otherwise the crystal with the highest signal is selected. A large value of $\delta_{E}$ is chosen when the N/S ratios are high and a small value of $\delta_{E}$ is chosen when the N/S is low. We assume that the energy resolution of the detector modules from counting statistics is $12 \%$ FWHM at $511 \mathrm{keV}$. While this value is optimistic, Comanor et al. [62] shows that the results are insensitive to this value.

Table 4.1 shows the results of the study. The Reject Multiples algorithm can give an extremely low MIF but only at the expense of EFF. The Second Highest Signal performs as well as the Maximum Signal but only when the noise is low, i.e. when 


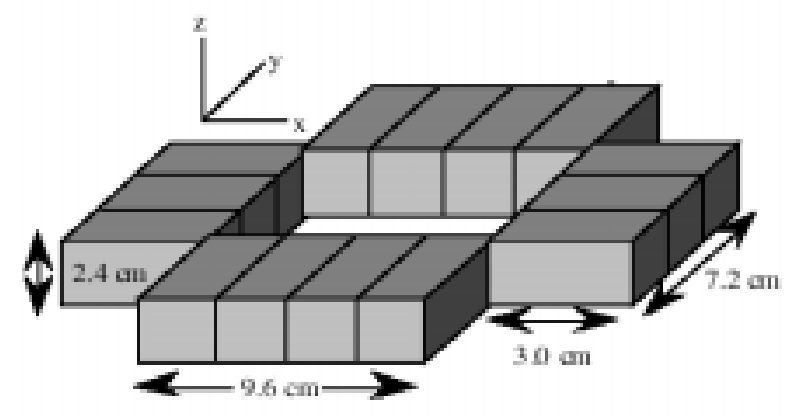

Figure 4.5: Simplified PEM Geometry with one module plane.

the N/S ratio is below 0.2. The Joint Algorithm performs like the Second Highest Algorithm at low N/S ratios if the appropriate $\delta_{E}$ is chosen. The ROI analysis does not distinguish one algorithm from another. The conclusion of this study is that merely taking the crystal with the highest signal as the interaction crystal does not have adverse effects on the reconstructed image. The crystal with the highest signal will be defined as the crystal of interaction.

\subsubsection{Simplified Camera}

Keeping matters simple at first, I simulate a simplified camera composed of detector elements with an attenuation constant of $\lambda_{\gamma}=1.2 \mathrm{~cm}$ and $100 \%$ photoelectric fraction. Fig. 4.5 shows the geometry of the simplified camera. It is equivalent to one detector module plane of the full camera. Only the $(8 \times 8)$ array of $(3 \times 3 \times 30) \mathrm{mm}^{3}$ crystals are shown. There is a $0.6 \mathrm{~cm}$ overlap between the detector banks to avoid gaps between the detector modules making the "patient port" $9.6 \times 6.0 \mathrm{~cm}^{2}$. While the camera is designed to allow the long banks to move in order to accommodate subjects of varying sizes, I only consider this setting of the camera. Optimizations for other aspect rations of the camera can be performed in a similar manner as I present here. 
In the present case of $2 \mathrm{D}$ simulation, I consider a detector that is only one module high, i.e. the axial extent of the camera is $\pm 1.2 \mathrm{~cm}$. However, since I reconstruct events that are detected by crystals in the same plane, I generate essentially in-plane events with the polar angle $\theta=\frac{\pi}{2} \pm \tan ^{-1} \frac{0.3 \mathrm{~cm}}{6.0 \mathrm{~cm}}$. The small deviation from the $x y$ plane is twice the largest angle that an event can have and still be detected by one crystal plane. Cross plane events are not included in the reconstruction.

As I mentioned in $\S 4.1 .2$, a true simulation of the PET data acquisition must include scatter in the object being scanned. However, I do not model the scatter in this dissertation and so I trace the pair of $511 \mathrm{keV}$ photons to the detector faces without any deviation from their original directions. The photons are then allowed to penetrate into the crystals with an attenuation length $\lambda_{\gamma}=1.2 \mathrm{~cm}$. I assume a $100 \%$ photofraction with all the energy of the photoelectron being deposited in only one crystal and at the point where the photon interacted. Scatter in crystal will be studied in later sections of this chapter. The generation of scintillation photons and the number detected by the PMT and the PD is also postponed until a more realistic simulation is done later in the chapter. In the meantime, the DOI information is determined by smearing the "true" interaction points by a Gaussian distribution along the major axis of the crystal. I use distributions with fixed widths of $0.0 \mathrm{~mm}$ (true interaction point), $5.0 \mathrm{~mm}$ and $10.0 \mathrm{~mm}$ FWHM.

\subsection{Reconstruction: Fourier Methods}

\subsubsection{Sampling}

One of the major challenges in reconstructing images for the PEM camera is the irregularity of its sampling. The extension of the FOV to include the entire region enclosed by the detector modules exacerbates this problem. Filtered Backprojection, the method of reconstruction which I employ in this thesis, assumes that the sinogram 


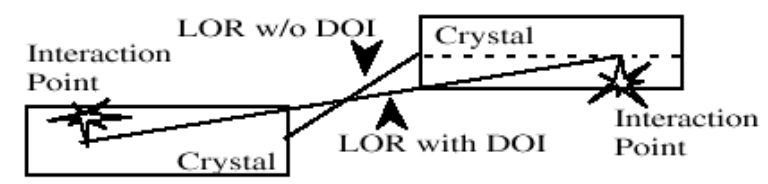

Figure 4.6: Definition of Chords with and without DOI.

data are sampled regularly in the radial direction and at uniform angular increments. Sinograms from circular PET cameras are in good agreement with these assumptions provided that the FOV from which the sinogram data are taken is limited to the central region of the camera. The PEM camera violates both assumptions. Radial sampling is only approximately regular when chords are defined between opposite sides (banks) of the camera. In addition, the angular sampling is nowhere regular.

Chords (or lines of response, LOR) without DOI are defined by connecting the front faces of crystals as shown in Fig. 4.6. This is the standard definition of chords in PET. The intrinsic sampling density in sinogram space of the camera is shown in Fig. 4.7. The FOV fills a region of sinogram space that resembles an hourglass. The irregular sampling of the tomographic data, both in $s$ and $\phi$, resulting from the rectangular geometry is readily apparent, even at the line $s=0$ where the sampling would be regular if the camera were circular.

In contrast, chords with DOI are defined as connecting the photon interaction points perpendicularly projected onto the long axis of the crystal. This is also shown in Fig. 4.6. Fig. 4.8 shows the sampling of the detector if the true DOI were measured. This scatter plot is produced by the Radon coordinates of events from a simulated flood source. We define chords with DOI using the long axis of the crystal because we have no information of the location of the interaction in the plane perpendicular to this axis. In keeping with the standard definition of chords in PET, this definition defaults to the center of the crystal face if the interaction occurred at or close to the face of the crystal. 


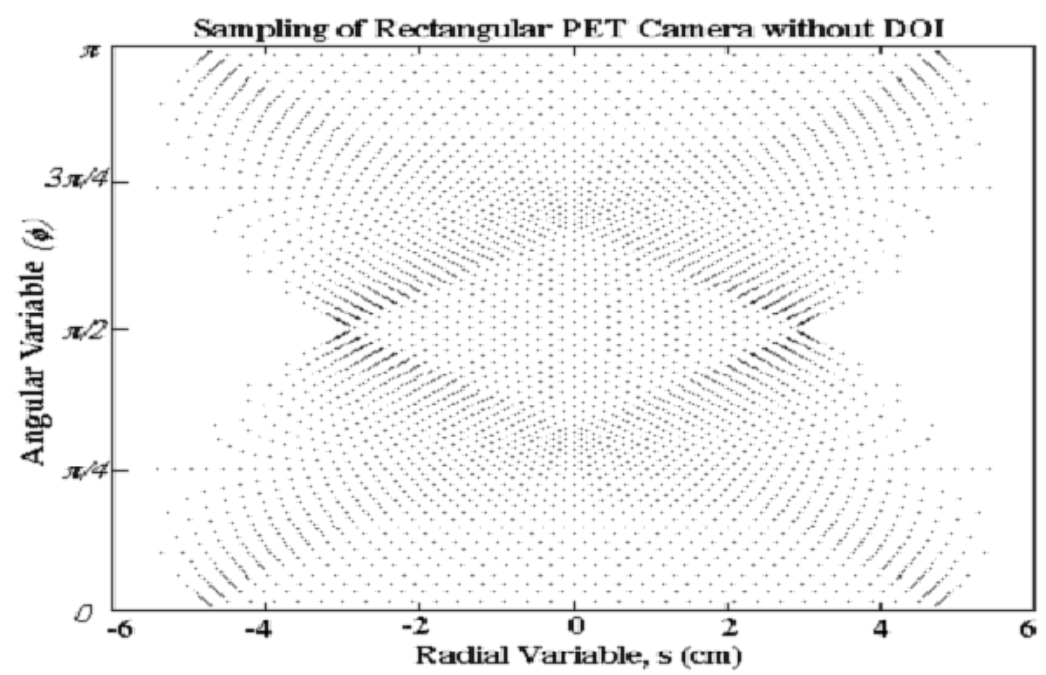

Figure 4.7: Sampling Density without DOI information.

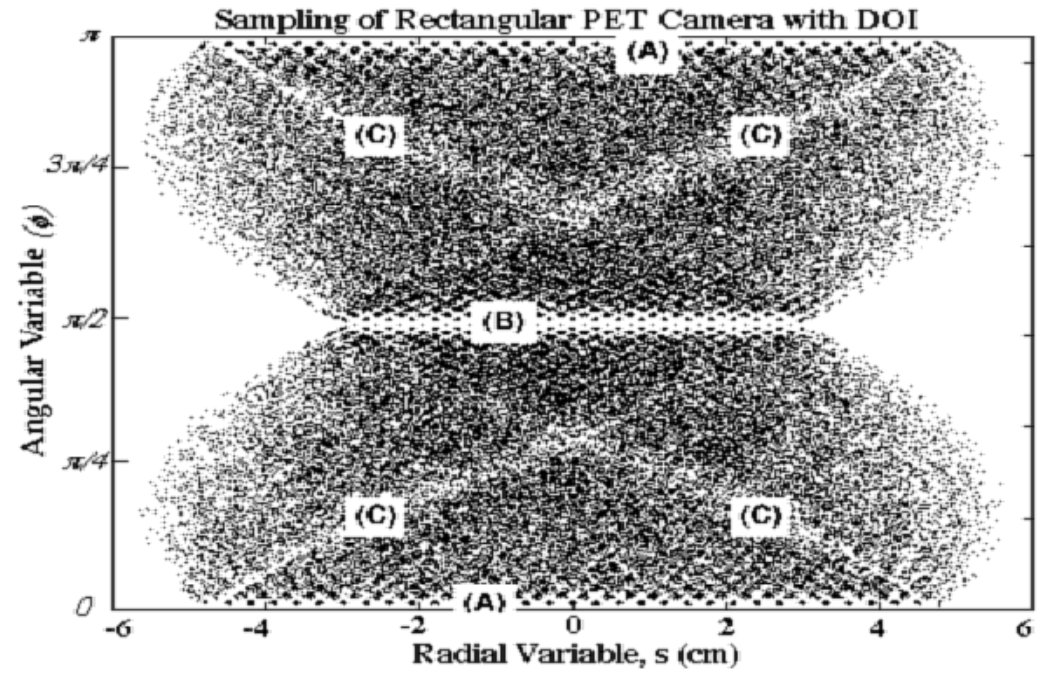

Figure 4.8: Sampling Density with DOI information. Regions (A), (B), and (C) are discussed in text. 
There are several distinct regions in the sinogram sampling using DOI (Fig. 4.8). Regions (A) and (B) near $\phi=0$ and $\phi=\frac{\pi}{2}$ respectively, represent chords formed by photons normally incident on the crystal faces. The DOI provides no new information because the interactions that occur in the same pair of crystals but different depths are mapped to the same chord and are thus degenerate ${ }^{1}$. Sampling in these regions is almost identical to that in Fig. 4.7. Note that DOI improves the sampling significantly even at small angles from $\phi=0$ and $\phi=\frac{\pi}{2}$. Region (C), the slightly dense areas that cross the hourglass, represent chords formed by crystals from corners of the camera. The detection efficiency is low in these regions because there is less detector material. This loss in efficiency can be corrected by normalization. The rest of the sinogram exhibits high sampling density.

Previous works have addressed the issue of irregular sampling in PET cameras. Camera motion and non-circular camera geometries [63] also result in irregular sampling of the sinogram. Circular cameras use a $\frac{1}{d}$ sampling rate where $d$ is the dimension of the crystals face. As mentioned in Chapter 1.3.2, this sampling rate actually increases away from the center of the tomograph. Camera motion is employed to increase the sampling density of the camera. Huesman et al. [64] use a clamming motion where the camera alternates between two positions, clam-open and clam-closed [65]. The sampling issue is often addressed by rebinning the acquired data into regularly sampled sinogram bins. Interpolation is often required to achieve evenly spaced sampling, effectively increasing the radial bin width of the sinogram and often degrading spatial resolution. The DOI information of the PEM camera increases the sampling density without camera motion and it allows us to rebin our data with minimal interpolation. However, as I will show, while the data from the camera can be binned in regular radial bins, angular binning is inherently irregular.

Regular radial binning is desirable in order to be able to use the Fast Fourier Transform (FFT). The FFT is an efficient calculation of the discrete Fourier trans-

\footnotetext{
${ }^{1}$ Degeneracies are different chords that lie on the same point in sinogram space.
} 
form of a sampled function provided that the samples are taken at regular intervals ${ }^{2}$. Recall that in the inversion of the Radon transform, the Fourier transform is only over the radial variable $s$. Thus, we only need to ensure that linear sampling is regular. Since computation time is a consideration in image reconstruction, I take advantage of the FFT and construct my sinograms with regular radial bins.

I study two angular sampling schemes: a) one whose angular sampling is as close to uniform angular sampling as possible, and b) one based on the grossly nonuniform pattern obtained from a rectangular camera with discrete detectors. The first sampling scheme is called Fixed Width (FW) scheme and the second is called Natural Width (NW) scheme. The DOI information is essential to these schemes as the challenge is to exploit the extra information where it increases sampling density while appropriately treating the sinogram regions where it does not.

\subsubsection{Sinogram Parameters}

The speed and simplicity of FBP make it a convenient choice as a reconstruction algorithm for the PEM camera. As shown in Fig. 4.8, continuous DOI all but removes the concern about irregular sampling. Discrete sampling only occurs in regions (A) and (B) which from hereafter I call "the problem regions." The high sampling density of the camera allows the use of quarter-crystal-width $(0.75 \mathrm{~mm})$ radial bins throughout most of the sinogram. For obvious reasons, this sampling scheme is called $\frac{d}{4}$ sampling. The choice of small bins allows the maximal use of the DOI information while the choice of regular sampling allows the use of the FFT in filtering of the sinograms. The small bin size also assures high resolution in the images. This choice of sinogram bin size leads to empty (zero efficiency) sinogram bins only near the problem regions and only when regular angular increments are used.

Zero efficiency bins $(\mathrm{ZEB})$ result in streak artifacts in images that radiate

\footnotetext{
${ }^{2}$ The other requirement that the number of samples be a multiple of two can be taken cared of by padding the data with zeros.
} 
out from point sources or that traverse the FOV in extended sources. At worse, they produce a cross-hatching effect in the reconstructed image. These artifacts are readily apparent in reconstructed images because of the filtering that is done during the reconstruction. ZEB in the midst of otherwise highly populated sinogram bins represent a high frequency component in the Radon transform of the object. This is evidenced by the sharp change in values between the ZEB and their neighbors. Recall that the filter used in 2D reconstruction is the Ramp Filter $f(k)=|k|$. This filter preferentially amplifies high frequency components while filtering out low frequency components.

Since these streak artifacts can be thought of as a high frequency noise, I can use an apodizing window on the Ramp filter to roll over the high-frequency response. This is the conventional solution for camera systems with low statistics (and therefore high levels of noise) which can have bins that mimic ZEB. However, this is equivalent to convolving the reconstructed image with a blurring function to smooth out the streak artifacts. This defeats our original goal of high resolution reconstruction. I choose to correct these bins in sinogram space instead of using a windowing technique in frequency space.

The use of the term "zero efficiency bin" is rather misleading. These bins have zero efficiency because of the definition of chords. If I choose not to confine the point of interaction to lie along the major axis of the crystal, all the bins should in fact be filled. However, this procedure is equivalent to smoothing the sinogram with a spatially variant kernel, which I will need to deconvolve out after the reconstruction. Deconvolving a spatially invariant kernel in $2 \mathrm{D}$ is computationally intensive (not to mention the fact that we will need to do this in $3 \mathrm{D}$ as well!). It is true that restricting the location of the interaction point to lie along the major axis of the crystal is also a form of spatially variant smoothing. However, there is "less" smoothing involved in this procedure than in that which allows the interaction points to be anywhere within the volume of the crystal. I will address this issue in Chapter 5.

For the same reason that small radial bins are used, namely to take full ad- 


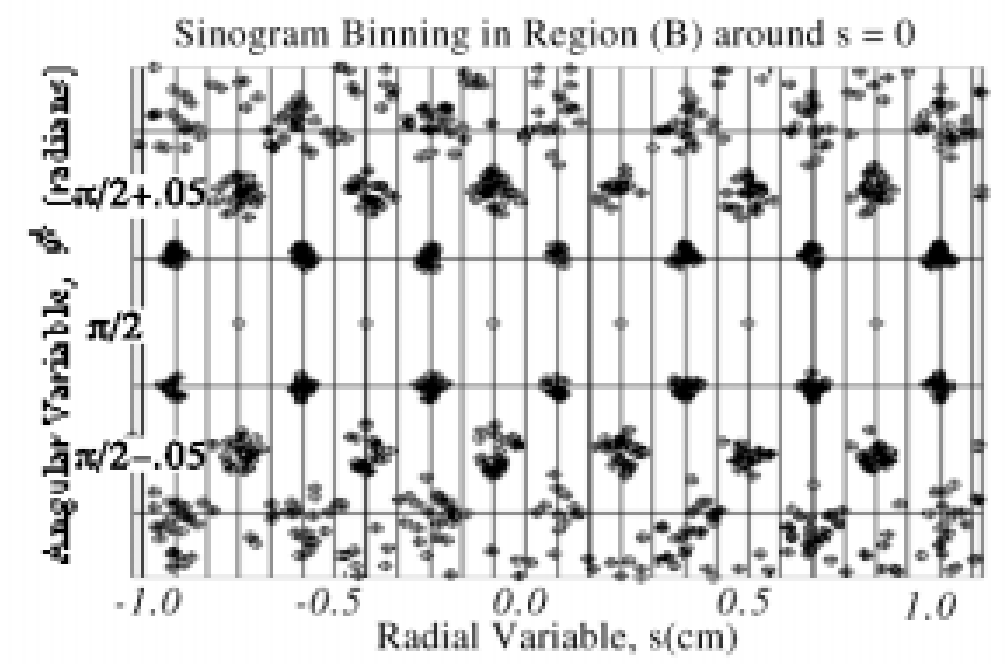

Figure 4.9: Sampling Density with DOI information in Region (B) of Fig. 4.8.

vantage of the DOI information, I use small angular bins to sample $\phi$. However care is needed in selecting the angular widths to minimize the number of zero-efficiency sinogram bins. I choose small angular bin widths in all the regions of the sinogram except around the problem regions where I use angular widths dictated by the geometry. I accommodate this choice of variable angular bin widths by multiplying each projection by a factor that is proportional to the angular width $\delta \phi$. Eqn. 4.6 describes this procedure where $f$ is the approximated image intensity at the current pixel $(i, j), F_{n}$ the $n^{t h}$ of the $N$ filtered projections, $r_{i j}$ the distance from the center of the tomograph to the pixel, and $\delta \phi$ is the angular width of the $n^{\text {th }}$ projection. The factor is ignored in normal FBP because it is a constant when the projections are taken at equal angular intervals.

$$
f\left(x_{i}, y_{i}\right)=\sum_{n=0}^{N-1} F_{n}\left(r_{i j} \cos \frac{n \pi}{N}+r_{i j} \sin \frac{n \pi}{N}\right) \delta \phi_{n}
$$

Without crystal penetration, the predefined angular bin widths in the prob- 


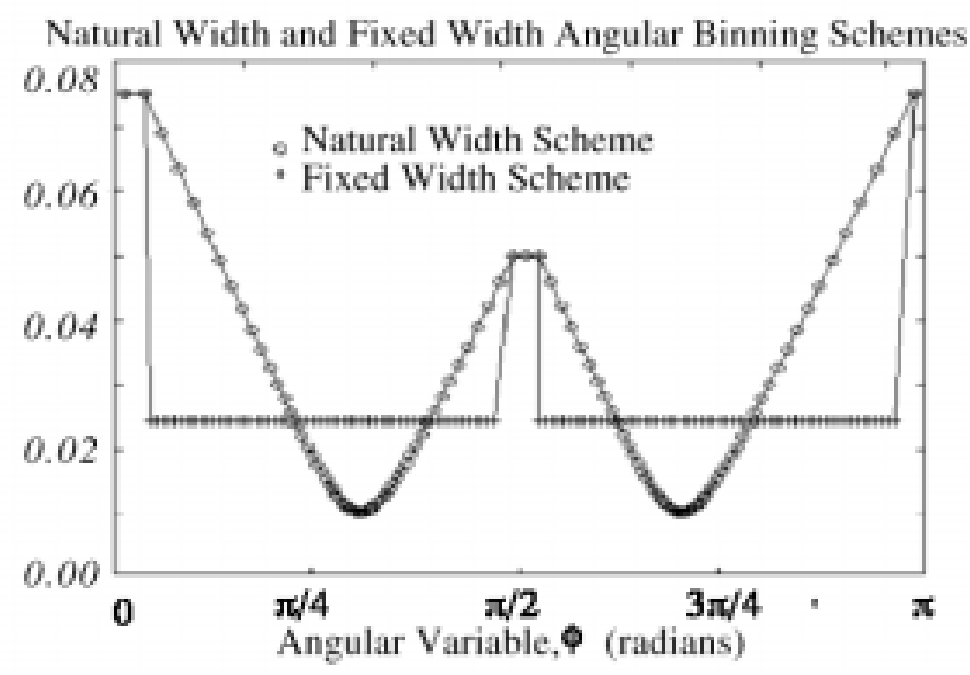

Figure 4.10: Angular Binning Scheme.

lem regions are defined by the crystal widths and the dimensions of the camera. With crystal penetration and DOI, however, these widths are slightly modified. Fig. 4.9 is an expansion of Region (B) in Fig. 4.8 with $s \in[-1.0,1.0]$. The boundaries of the angular sampling are chosen to bisect the clusters of points, yielding $\delta \phi_{\frac{\pi}{2}}=0.050$. A similar procedure used around $\phi=0$ requires that $\delta \phi_{0}=0.075$. These values are rounded to the nearest thousandths for simplicity. Fig. 4.9 also shows the $\frac{d}{4}$ sampling of the $s$. All the sinogram bins are populated with the exception of the degenerate chords which lie on bin boundaries. A similar pattern but with different spacing occurs at $\phi=0$. Half of the events from these chords are assigned to each of the bins upon whose boundaries they lie. This simple procedure of minimal interpolation leaves no unpopulated sinogram bins.

We construct two angular binning schemes to bin the events in the regions of dense sampling. The Natural Width scheme (NW) uses half the widths of the intrinsic sampling angles of the camera at $s=0$ (Fig. 4.7). The widths are then 


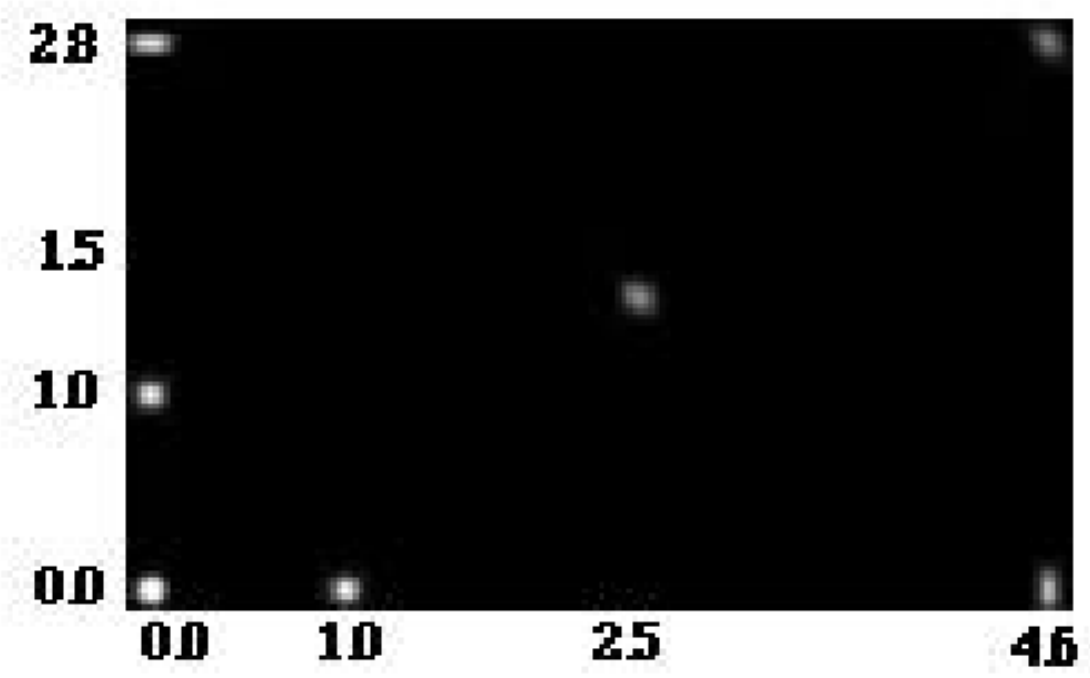

Figure 4.11: PSF Reconstruction.

smoothed (except at $\phi=0, \frac{\pi}{2}$ ). The fixed width scheme $(\mathrm{FW})$ uses a fixed angular width in the densely samples regions. At the problem regions, we use three angle bins with the predetermined widths. This gives a total of 120 angular bins for both binning schemes. Fig. 4.10 shows the angle bin widths as a function of the sampling angle for both the NW and FW binning schemes.

\subsubsection{Reconstructed Images and Results}

Before reconstruction, the sinograms are normalized to correct for sampling and geometric efficiency variation in the camera. As I discussed in Chapter 2, PET data is the Radon transform of the object in the FOV in the limit of inifinitely small detectors with $\mu=0 \mathrm{~cm}$. In order to correct for the finite size and attenuation length of the detector, each projection bin can be multiplied by a normalization factor. This normalization factor can be calculated by taking PET data from a source with a known Radon transform and performing a projection-bin-by-projection-bin 


\begin{tabular}{cccc}
\hline \hline Location & $0 \mathrm{~mm}$ & $5 \mathrm{~mm}$ & $10 \mathrm{~mm}$ \\
\hline$(0.0,0.0)$ & $1.74(0.06)$ & $1.90(0.05)$ & $1.90(0.08)$ \\
\hline$(1.0,0.0)$ & $1.73(0.04)$ & $2.15(0.13)$ & $2.51(0.28)$ \\
\hline$(0.0,1.0)$ & $1.74(0.05)$ & $2.17(0.04)$ & $2.52(0.22)$ \\
\hline$(2.5,1.5)$ & $2.03(0.15)$ & $2.35(0.06)$ & $2.67(0.10)$ \\
\hline$(4.6,0.0)$ & $1.85(0.45)$ & $2.81(0.12)$ & $2.70(0.33)$ \\
\hline$(0.0,2.8)$ & $1.83(0.46)$ & $2.70(0.08)$ & $2.50(0.09)$ \\
\hline$(4.6,2.8)$ & $2.05(0.35)$ & $2.51(0.14)$ & $2.50(0.15)$ \\
\hline \hline
\end{tabular}

Table 4.2: PSF Resolution for Various DOI Resolution.

comparison. The normalization is calculated by comparing a flood source sinogram with the Radon transform of a box of the appropriate dimensions. We use filtered backprojection with a ramp filter for reconstruction. In the FW angular binning scheme, the projection data with the wider widths at $\phi=0$ are backprojected with $\frac{1}{3}$ of the value at $\phi=\frac{-0.075}{3}, 0, \frac{0.075}{3}$. The same is done for the other two wider width projection data on either side of $\phi=0$. Similarly, the projection data with the wider widths at $\phi=\frac{\pi}{2}$ are backprojected twice with $\frac{1}{2}$ of the value at $\phi=\frac{\pi-0.050}{2}, \frac{\pi+0.050}{2}$. The same is done for the other two wider width projection data on either side of $\phi=\frac{\pi}{2}$. The widths at $\phi=0$ and $\phi=\frac{\pi}{2}$ are approximately thrice and twice the small fixed width respectively.

Fig. 4.11 shows a set of reconstructed images of point sources of equal intensities in various positions in the FOV. Perfect DOI resolution was assumed in the data and FW angular binning scheme was used in building the sinogram. Shown is the top right quadrant of the camera with the center of the FOV at $(0,0)$. Table 4.2 lists the resolutions of the PSF. The variation in the number gives an indication of the symmetry of the PSF, i.e. the smaller the variation, the more circular the shape. The shapes of the PSF vary from circular at the center of the FOV to elliptical at the corners and near the edge. The PSF's near the edge have FWHM of $2.5 \mathrm{~mm}$ along the long axis and as small as $1.0 \mathrm{~mm}$ along the short axis. The long dimensions are 


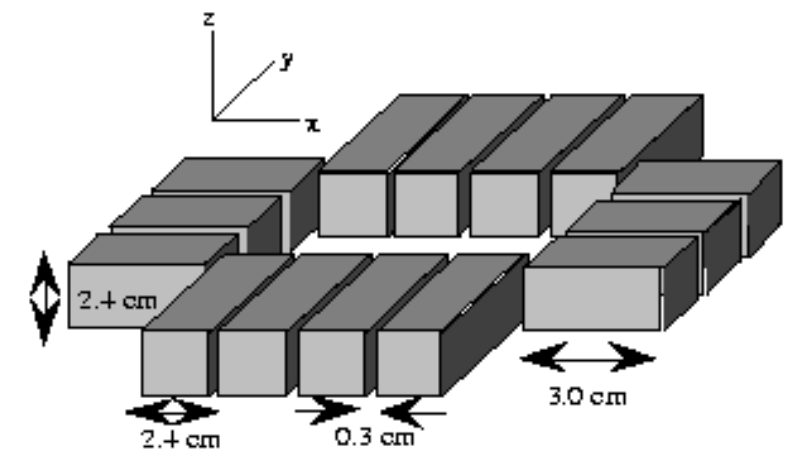

Figure 4.12: Realistic PEM Geometry with one module plane.

consistent with a $3 \mathrm{~mm}$ crystal width while the short dimensions are the result of the DOI information. The apparent variation in the intensity of the sources is consistent with the variation in their FWHM's. ROI's drawn around each point source have equal activity.

I did not observe significant differences between the images reconstructed with the FW versus the NW angular scheme as long as the sampling in the problem areas is done using the predetermined widths. Hereafter, I will present images reconstructed with the FW binning scheme as it is easier to implement.

\subsection{Realistic Camera}

Having developed a reconstruction algorithm for a simplified detector, I now add real world effects into the simulation. I include $0.5^{\circ} \mathrm{FWHM}$ acollinearity between the photons and model fully-3D Compton scattering in the detector modules. I use the properties of LSO in Fig. 4.3 to model scattering and attenuation. When energy is deposited in more than one crystal, as in the case when the $511 \mathrm{keV}$ Compton scatters outside of the initial crystal, the crystal with the largest signal is chosen to be the crystal of interaction. Patient scatter in the FOV and positron range are still 
not simulated. Reconstructions are limited to $2 \mathrm{D}$ so cross plane events are not used. The overall energy resolution is $17 \%$ FWHM at $511 \mathrm{keV}$.

The DOI is measured as described in $\S 3.3$ and $\S 4.1 .2$. The depth estimator $\Gamma$ is defined as the ratio of the PD signal and the sum of the PD and PMT signals. It varies linearly with the DOI and I assume that it takes on values of .25 to .75 across the $3.0 \mathrm{~cm}$ length of the crystal. Using Eqn. 4.5, I calculate the DOI from $\Gamma$ by

$$
z=L_{\text {crystal }} \frac{\alpha-(\alpha+1) \Gamma}{\alpha-1} .
$$

Because of noise, however, $\Gamma$ may take values outside $[.25, .75]$ which will indicate a DOI outside the volume of the crystal. In these cases, I assign the depth to the appropriate end of the crystal, i.e. $z=0$ when $\Gamma>.75$ and $z=L_{\text {crystal }}$ when $\Gamma<.25$. I will return to this issue in Chapter 5. The depth is discretized into one of eight DOI bins to model the DOI measurement resolution. Huber et al. [66] reports on the characterization of modules which I model.

In addition to the effects I have enumerated, I place one-crystal gaps between the detector modules in anticipation of packing limitations in the camera. The $2 \mathrm{D}$ acceptance of the camera drops by $10 \%$ due to the gaps. Fig. 4.12 shows the geometry of the realistic camera.

\subsubsection{Sampling Density and Sinogram Parameters}

Fig. 4.13 shows the sampling density of the realistic detector. The discrete DOI bins lead to an increase in the degeneracies in the sinogram. The $3 \mathrm{~mm}$ gaps between the modules lead to unsampled x-shaped regions around $\phi=0$ and $\phi=\frac{\pi}{2}$. The gaps in the corners result in unsampled regions around the $(\mathrm{C})$ region of Fig. 4.8. DOI information minimizes the effect of the module gaps as the unsampled regions would have covered more of the sinogram if DOI were not measured.

Many of these problems are caused by DOI discretization. I address this by adding noise to the calculated and discretized DOI to make it continuous again. Since the interaction point is assigned to the center of the DOI bin, I add a length to 


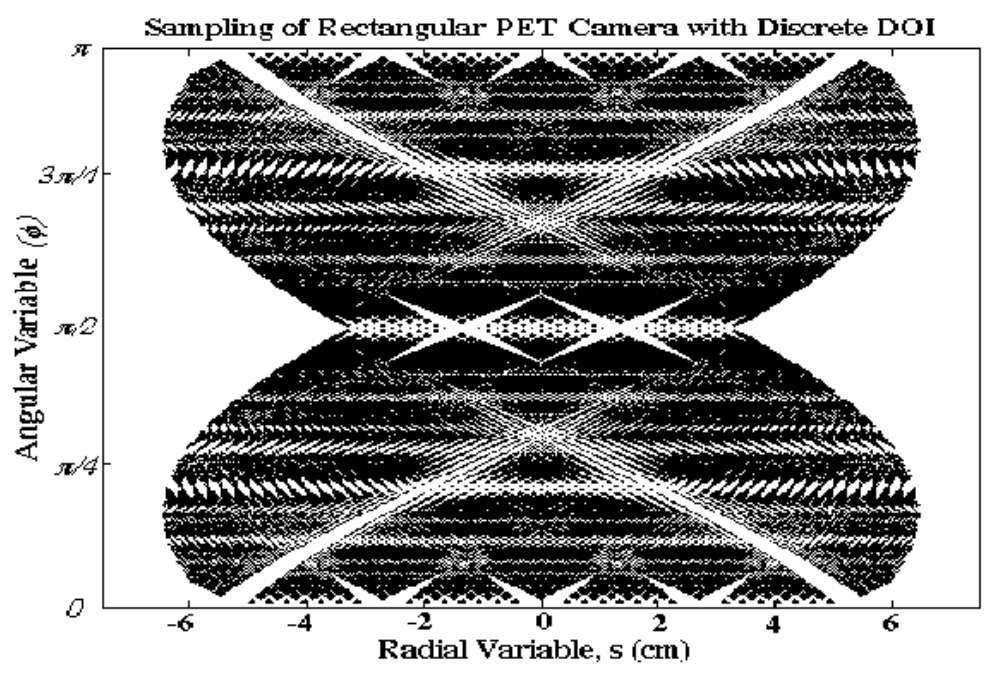

Figure 4.13: Sampling density of realistic camera. The sampling points are calculated from chords formed by connecting each DOI bin in each crystal with DOI bins from other crystals.

the DOI drawn from the flat distribution $[-1.875,+1.875] \mathrm{cm}(30 \mathrm{~cm} / 8$ DOI bins $)$. The DOI takes a continuous random value within the boundary of the discritized DOI bin. The effect is to smooth the sinogram: more where it needs it and less where it does not. The procedure results in a sinogram with which we can again use the same angular binning schemes that we determined for the simplified camera. Fig. 4.14 shows a sinogram from a flood phantom after the DOI is smoothed. The dimensions of the realistic camera are slightly larger than the ideal one but the differences result in only small changes in the predetermined angles and widths at these angles.

The redistribution of events that I performed on the $\phi=0, \frac{\pi}{2}$ now needs to be done on a few more places in the sinogram. The procedure that I use is the following:

- For each angular bin, locate the first and the last non-zero radial bins.

- Between these two bins, locate all the bins which have counts that are lower 


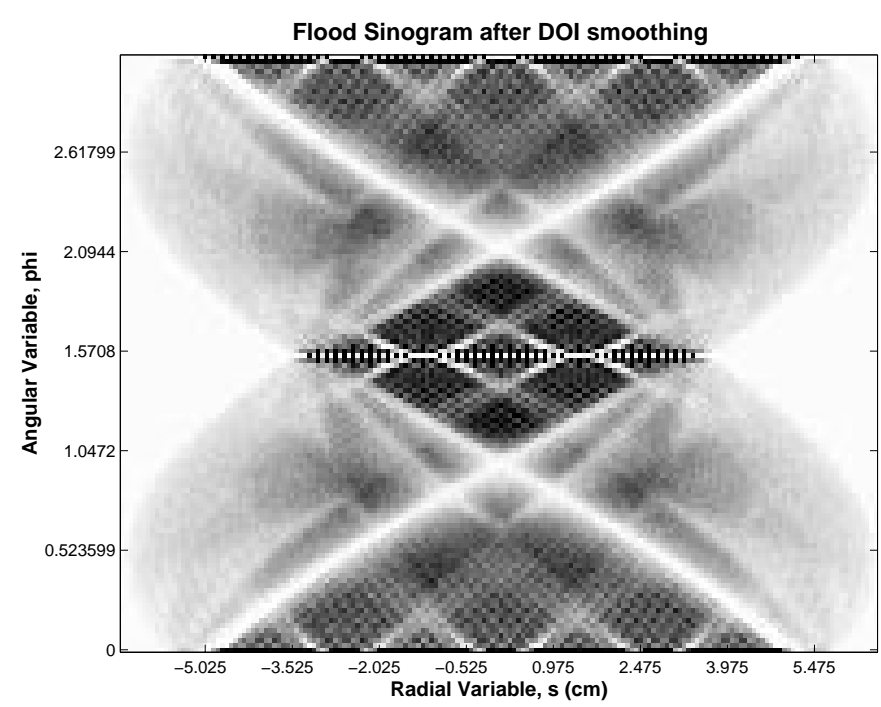

Figure 4.14: Sampling density of realistic camera after DOI smoothing. A small random value is added to each DOI so that it becomes a random continuos variable within the boudaries of each DOI bin.

than a set fraction than their adjacent bins. Define these bins as ZEBs.

- For each of the ZEBs, choose the bin on either side that has the higher event count and assign to the ZEB and the chosen bin the average count of the two bins.

- Mark any modified bin, ZEB or otherwise, so it is modified at most once.

This procedure can be used several times to eliminate ZEBs. Note that I have redefined a ZEB such that instead of having a "hard 0," it is any bin that has a value that is below a fraction of the number of counts in an adjacent bin.

I reconstruct data from the realistic camera using a method similar to that developed for the simple detector in spite of the fact that the real-world effects exacerbate the sampling problems. The distribution of unsampled regions in the sinogram is such that there is not a set angular bins (such as the FW or the NW) 


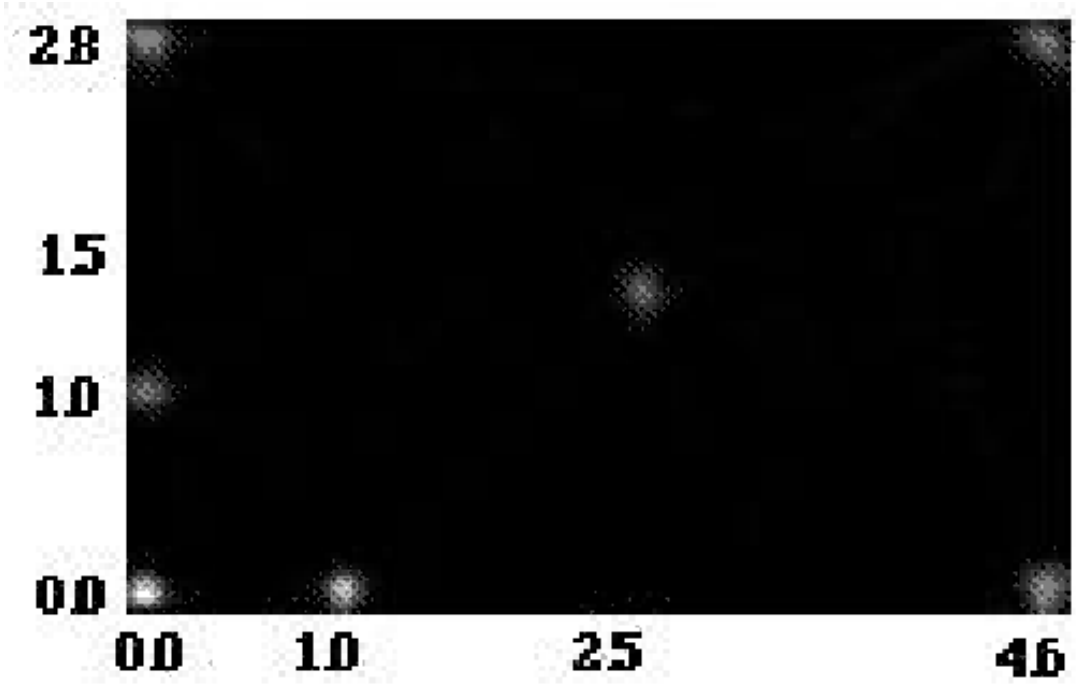

Figure 4.15: Reconstructed point sources within realistic camera. Shown is the right quadrant of the FOV. Axes are in units of $\mathrm{cm}$.

that will easily eliminate ZEBs. In addition, the locations of the poorly sampled regions depend on the distance between opposing detector banks which is adjusted for each patient. It would be desirable to use an algorithm which is not sensitive to the changing camera dimensions.

\subsubsection{Reconstruction and Results}

The reconstruction of the sinogram of the realistic camera is essentially the same as in the simplified camera except that in the realistic camera, ZEB persist even after the redistribution of the sinogram data between radial bins as prescribed in $§ 4.2 .3$. These ZEB are the result of gaps in the corners of the camera, between the adjacent detector banks. The ZEB resulting from the gaps between modules of the same bank are eliminated by the addition of noise to the DOI. To remedy the situation, I use the Iterative Reconstruction Reprojection (IRR) developed by Medoff et al. [67] to estimate the missing data. The IRR algorithm first reconstructs the 


\begin{tabular}{cc}
\hline \hline Location & PSF FWHM \\
\hline$(0.0,0.0)$ & $1.92(0.16)$ \\
\hline$(1.0,0.0)$ & $2.14(0.13)$ \\
\hline$(0.0,1.0)$ & $2.37(0.13)$ \\
\hline$(2.5,1.5)$ & $2.42(0.10)$ \\
\hline$(4.6,0.0)$ & $2.81(0.21)$ \\
\hline$(0.0,2.8)$ & $2.44(0.22)$ \\
\hline$(4.6,2.8)$ & $2.79(0.23)$ \\
\hline \hline
\end{tabular}

Table 4.3: PSF Resolution for Various DOI Resolution.

image from the incomplete sinogram and then, using a priori information in the form of constraints, forward projects the reconstructed image into the gaps to estimate the values of the missing information. I use the positivity constraint, i.e. setting all negative pixels to zero, in the intermediate images as a priori information. Iteration of this process gives the final image.

Fig. 4.15 shows the reconstructed image of the point sources in the same location as in the ideal camera, again reconstructed using the FW angular binning scheme. I performed only one iteration of the IRR algorithm since the image quality did not change dramatically with subsequent iterations. Table 4.3 lists the FWHM of the point sources at various locations in the FOV of the realistic camera. The apparent differences in intensity of the point sources are due to the variation in the sizes of the reconstructed PSF. The intensities are proportional to the inverse of the the square of their width. ROI's placed around these points yield identical activities.

Fig. 4.16 shows a reconstruction of three extended sources and a point source. The image represents the entire FOV of the camera. I simulated a circle and two ellipses of various activities at various locations in the FOV. The circle has a radius of $0.5 \mathrm{~cm}$ while the semi-minor and semi-major axes of the ellipses are $(0.5,1.25) \mathrm{cm}$ for the vertical ellipse and $(0.5,2.25) \mathrm{cm}$ for the horizontal ellipse. The simulated relative activities, in the order circle:vertical ellipse:horizontal ellipse are 


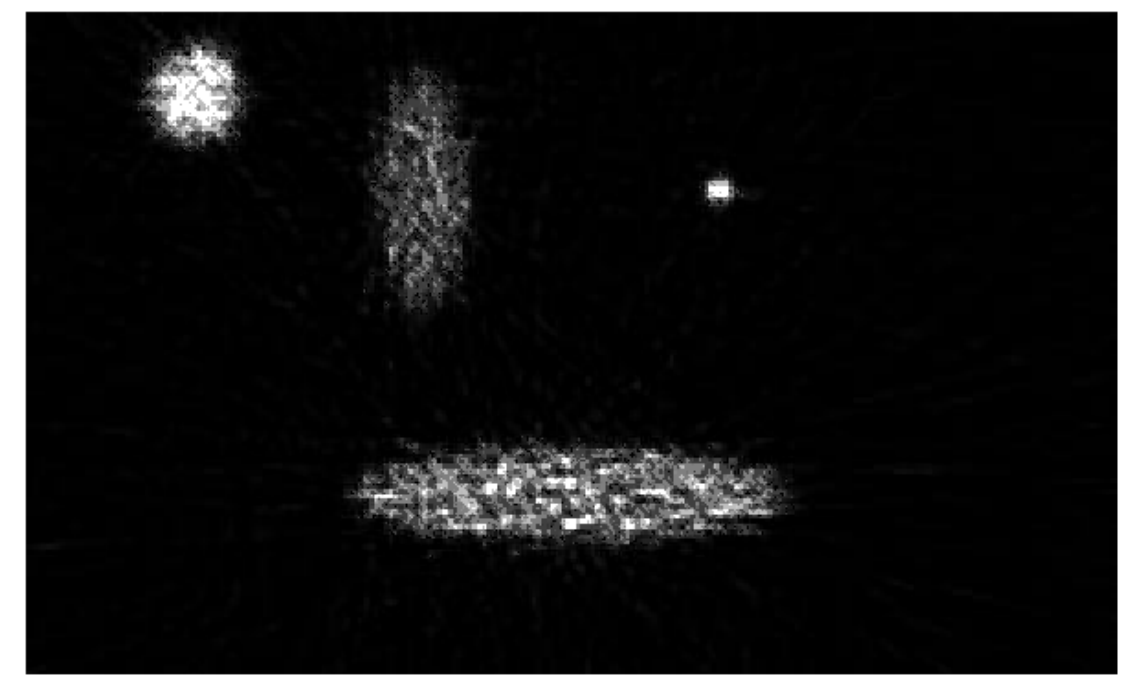

Figure 4.16: Reconstructed extended sources within the realistic camera (a circle, two ellipses and a point sources). Image represents the entire FOV of the camera.

1.00:0.63:0.73. The image represents $10^{6}$ annihilation events. The shapes of the source structures are sharp and their activities are relatively constant within their boundaries. The relative activities are consistent with the inputs to the simulation. I have included a point source to the right of the vertical ellipse which has a reconstructed FWHM of 2.30(0.20) mm, consistent with Table 4.3.

\subsection{Summary of 2D Reconstruction Procedures}

The following is a list of the procedures that need to be done in order to successfully reconstruct 2D images using data from the PEM camera. In this list, I do not assume that the aspect ratio of the PEM camera is as I have described in this chapter. After all, the long sides of the camera are designed to move. If the aspect ratio were as I describe, then the optimal values of the parameters and variables that I mention are stated in this dissertation. 
- Determine a set of azimuthal sampling angles $\phi_{i}$ and angular bin widths $\delta \phi_{i}$ that will minimize ZEBs. In particular, the sampling widths at the degenerate directions $\phi=0, \frac{\pi}{2}$ needs to be carefully chosen.

- Add a random value within the boundaries of a DOI bin to each event's DOI and calculate the Radon variables $\mathbf{s}$ with the modified interaction point.

- Calculate normalization values. I have used the ratio of flood phantom data and the Radon transform of a rectangle to calculate my normalization values. Note that the nearest neighbor smoothing that will be done on the data needs to be done on the high statistics data that will be used to calculate the normalization values.

- Set the data in all projection bins with normalization values above a specified threshold to 0 . The threshold is determined by inspecting the distribution of normalization values. A drop of two orders of magnitude in the distribution may indicate an appropriate threshold.

- Perform nearest neighbor smoothing on the data in the regions of ZEBs, making sure that each projection bin is modified at most once. Normalize the data with pre-calculated normalization file.

- Filter the sinogram in the Fourier domain. I have used the Ramp filter with sharp edges.

- For each projection direction, $i$, calculate $w_{i}=\frac{\delta \phi_{i}}{\delta \phi_{m i n}}$, the ratio of its angular bin width and the minimum angular width. Let $N_{i}$ be the round off value of $w_{i}$.

- Multiply the $i$ th projection by $\frac{w_{i}}{N_{i}}$ and backproject at $\phi_{i j}^{\prime}=\phi_{i}-\frac{\delta \phi_{i}}{2}+\left(j+\frac{1}{2}\right) \frac{\delta \phi_{i}}{N_{i}}$, $j=0 . .\left(N_{i}-1\right)$. 
- Perform the IRR by setting all negative pixels to zero and forward projecting the reconstructed image. Substitute the value of the forward projected data for all the ZEBs in the original normalized sinogram.

- Repeat the procedure starting from filtering of the "filled-in" sinogram until object is reconstructed. Simulations indicate that one iteration will be sufficient. 


\section{Chapter 5}

\section{Reconstruction in 3D}

This chapter discusses the 3D Reconstruction for the PEM Camera. There is one overriding reasons for extending PET imaging to 3D. A camera able to operate in fully-3D mode has higher sensitivity compared to a camera that is limited to 2D-imaging. Table 5.1 shows typical gains in geometric acceptances for conventional PET cameras (cylindrical) and the PEM camera by operating in 3D mode. Two cylindrical PET geometries are shown. In one configuration, the radius is twice the height of the cylinder, which is most common in current whole-body PET cameras. In the other configuration, the height is equal to the radius. High resolution cameras optimized for head imaging are currently being proposed and built with this geometry [12]. The acceptance increases by at least an order of magnitude for all but the flood source in the PEM camera which increases by a factor of eight.

Higher acceptance translates to higher number of events which means better image statistics. The single largest component of image resolution is crystal size and with the trend for smaller and smaller crystals, the physical limit of $0.2 \mathrm{~cm}$ resolution, for FDG PET, is nearly realized in 2D imaging. Thus, the gain in $3 \mathrm{D}$ imaging is not in resolution but rather in improved image statistics. The benefit of using 3D PET is in the quieting of noise in historically event-starved PET data.

Higher acceptance can also mean shorter scanning times and lower radiation 


\begin{tabular}{llcc}
\hline \hline Detector & Simulated Phantom & 2D Acceptance & 3D Acceptance \\
\hline Cylindrical $2 h=r$ & Point source at center & $0.75 \%$ & $24.35 \%$ \\
Cylindrical $2 h=r$ & Flood source & $0.95 \%$ & $15.36 \%$ \\
\hline Cylindrical $h=r$ & Point source at center & $0.75 \%$ & $44.74 \%$ \\
Cylindrical $h=r$ & Flood source & $0.95 \%$ & $28.40 \%$ \\
\hline PEM & Point source at center & $3.75 \%$ & $62.02 \%$ \\
PEM & Flood source & $4.98 \%$ & $38.75 \%$ \\
\hline \hline
\end{tabular}

Table 5.1: Geometric Acceptance of Detectors.

dosage to the patient. Since the acceptance is increased, for a fixed amount of FDG, a larger fraction of the total number of annihilations become available as data. This allows for the possibility of decreasing the amount of FDG injected into the patient which translates to a lower radiation dose. In addition, it takes a shorter amount of time in $3 \mathrm{D}$ scans to acquire the same number of events as with $2 \mathrm{D}$ scans. Shorter scanning times mean less inconvenience to the patient, and a decreased likelihood that the object being scanned will move. Object motion during a scan can lead to inconsistent data and therefore difficult to reconstruct. At best, the motion will result in further blurring of the reconstructed image.

Current PET cameras are in fact capable of operating both in $2 \mathrm{D}$ and 3D modes, acquiring in 3D mode by retracting the septa between detector planes. Typically, a 2D scan is performed to measure the attenuation map that is used to correct for object attenuation in the 3D data acquired when the septa are retracted. The PEM camera, however, will not have septa and therefore has to operate in complete 3D mode. Fig. 5.1 shows the a frame model of the geometry of an idealized PEM camera, analogous to that in Fig. 4.5 for the 2D case. 


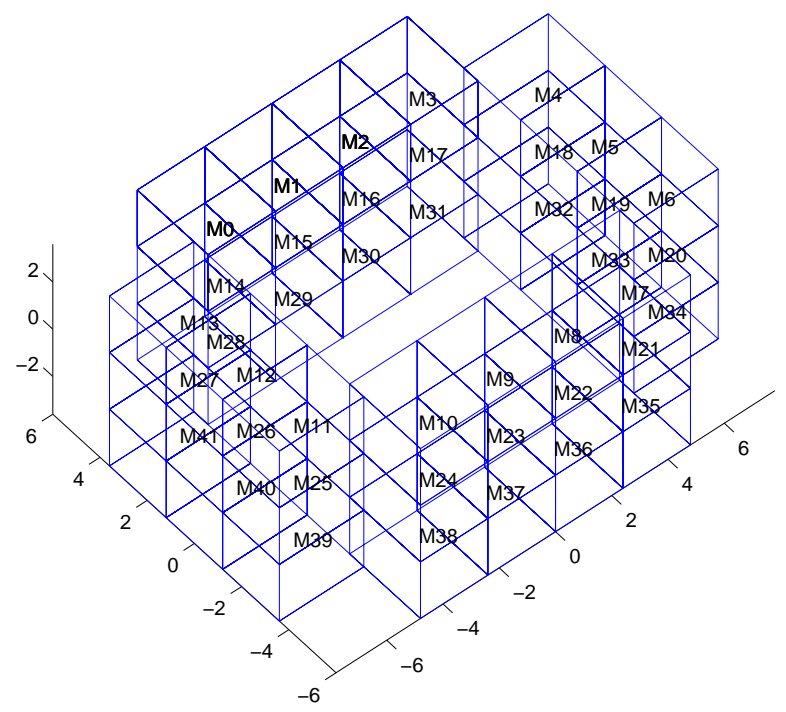

Figure 5.1: Wireframe image of idealized PEM Detector Geometry. Longest axis is the $x$ axis while axial direction through the FOV is the $z$ axis. Modules are grouped together in banks corresponding to the four sides of the rectangular FOV. The axes are in units of $\mathrm{cm}$. 


\subsection{X-ray Transform}

The use of the Radon Transform in 2D reconstruction is quite curious in that most of the development in reconstruction tomography occurred before the connection between the Radon transform and the reconstruction problem was established. Bracewell [24] gave the first solution to image reconstruction in the context of mapping the microwave radiation from the sun. Much of the early advancements in reconstruction tomography can be drawn from this work. However, when it became clear that the continuous inverse problem has been solved by Radon, virtually all of reconstruction tomography was cast in relation to the Radon transform. Thus, today, discussions regarding image reconstruction usually begin with the Radon transform.

However, 3D reconstruction does not involve the 3D Radon transform. Instead, it involves a related transformation: The X-ray transform. Up to a rotation, the 2D Radon transform corresponds to the X-ray transform. Our discussion of the Radon transform in Chapter 4 is nevertheless useful because virtually all the properties of the Radon transform applies to the X-ray transform as well. The inversion of the X-ray transform also closely follows the formulae of the Radon transform inversion.

The X-Ray transform is defined as

$$
\mathcal{P} f\left(\mathbf{u}=\mathbf{x}^{\perp}, \omega\right)=\int_{\mathbf{R}} d t f(\mathbf{x}-t \omega) ; \omega \cdot \mathbf{x}=0 .
$$

I have explicitly labeled the Cartesian coordinates of the transform as $\mathbf{x}^{\perp}$ to indicate that it is perpendicular to $\omega$, the unit vector in the direction of the projection. Note that $\operatorname{dim}(\mathbf{u})=\operatorname{dim}(\mathbf{x})-1$. Fig. 5.2 shows the coordinate system used for the 3D $\mathrm{x}$-ray transform. For this thesis, the parametrization of the transform that I use is the following

$$
\mathcal{P} f(u, v, \theta, \phi)=\int_{\mathbf{R}} d t f\left(\left[\begin{array}{ccc}
\cos \theta \cos \phi & -\sin \theta & \sin \theta \cos \phi \\
\cos \theta \sin \phi & \cos \theta & \sin \theta \sin \phi \\
-\sin \theta & 0 & \cos \theta
\end{array}\right]\left[\begin{array}{l}
u \\
v \\
t
\end{array}\right]\right)
$$




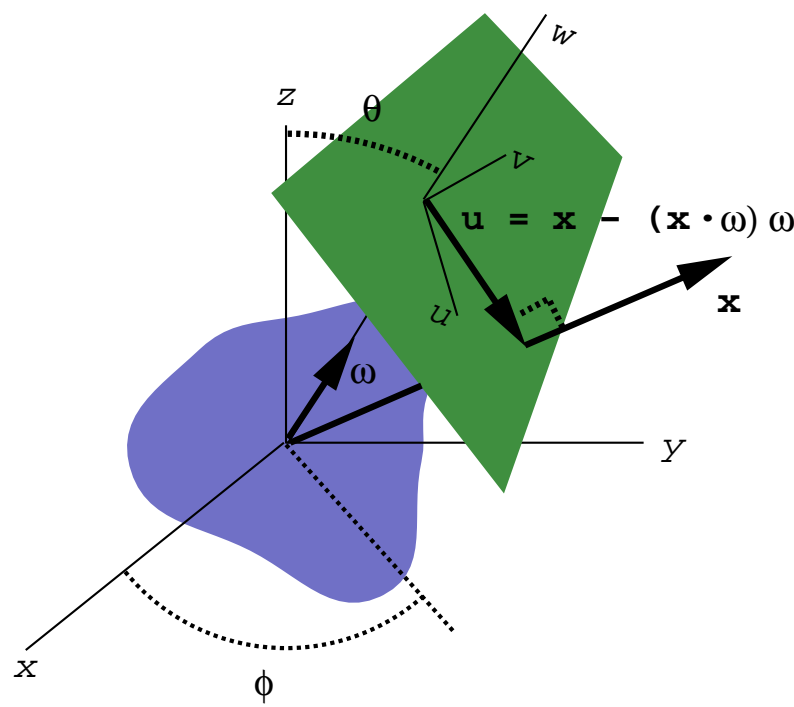

Figure 5.2: Coordinate System Used for 3D X-ray Transform.

Note that this is simply the integration of the function $f$ along a rotated $z$-axis $t$.

The projection data are a function of four variables. Each polar and azimuthal angle defines a plane within which two other variables are required to specify the point at which the function is evaluated. This means that the data set in $3 \mathrm{D}$ reconstruction is redundant in the sense that while it requires only three parameters to determine the value of the function in coordinate space, it requires four parameters to determine its value in projection space. Recall our discussion in the inversion of the Radon transform that the reconstruction of a 3D object maybe done by stacking $2 \mathrm{D}$ reconstructions of the object. The redundancy in $3 \mathrm{D}$ projection data makes this possible. As we shall see, this redundancy will allow us to bypass some of the problems that we have encountered in reconstructing images in $2 \mathrm{D}$.

Fig. 5.3 shows a subset of the projections of a parallelepiped that has the same dimensions as the FOV of the PEM geometry. The top left image (orientation is landscape) is the projection at $\theta=\frac{\pi}{2}$ and $\phi=0$., i.e. the projection along the 

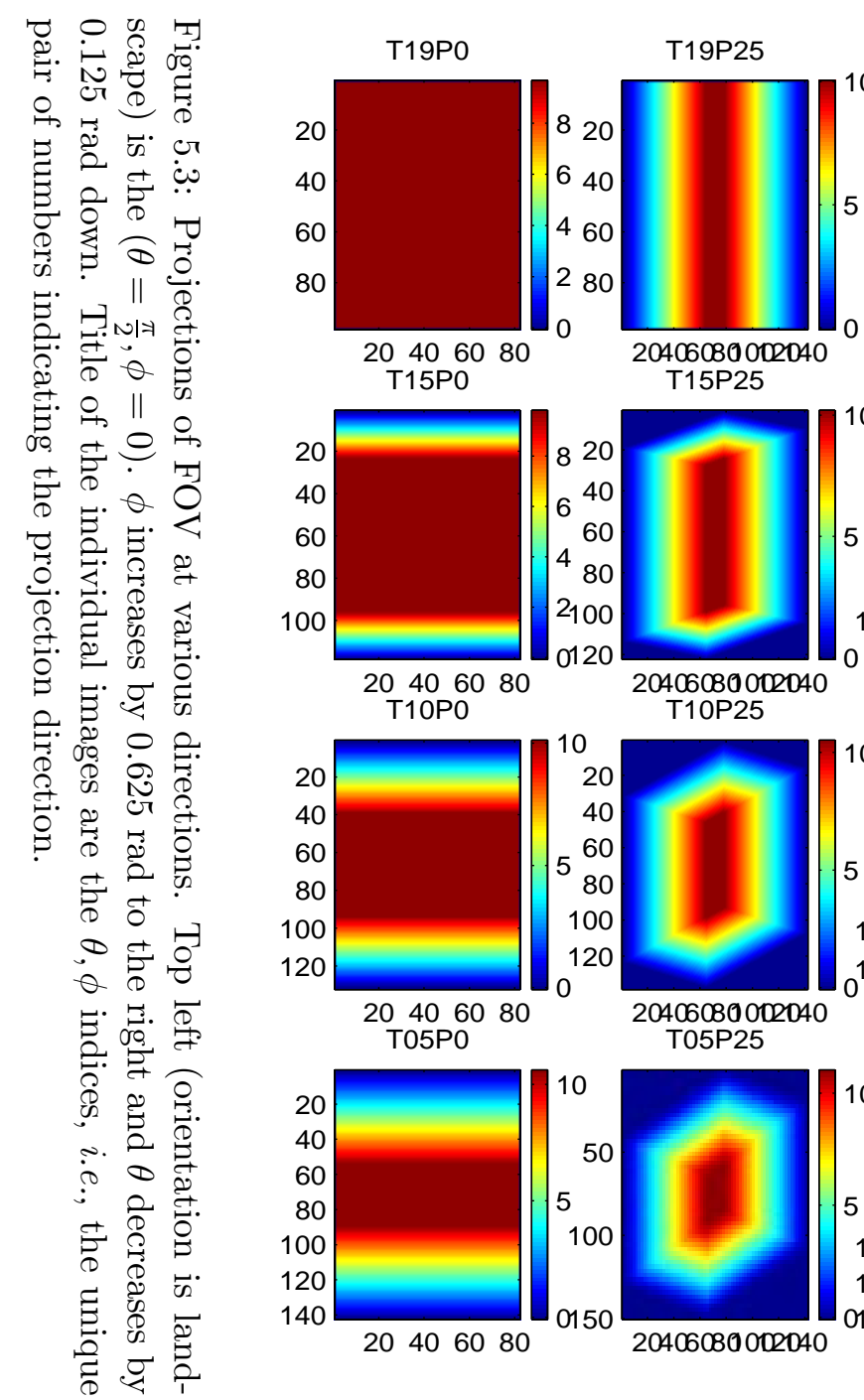

T19P45

T19P65

T19P85

T19P105
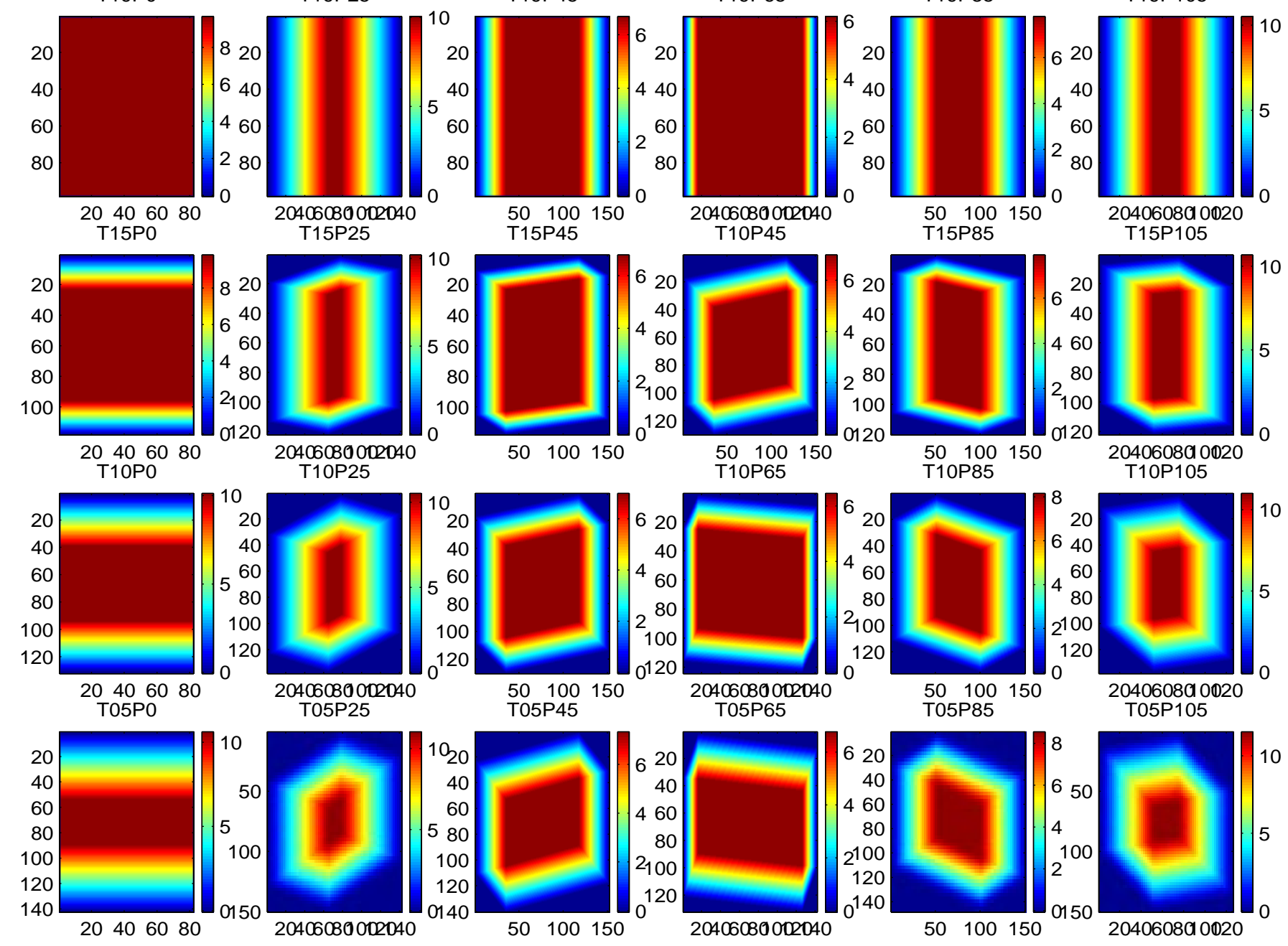
$x$-axis. Moving to the right increases $\phi$ by approximately $0.625 \mathrm{rad}$ and moving down increases $\theta$ by approximately $0.125 \mathrm{rad}$.

The Radon transform and the X-ray transforms are related by the following equation:

$$
\mathcal{R} f(s, \xi)=\int d \mathbf{u} \mathcal{P} f(\mathbf{u}, \omega) \delta(s-\mathbf{u} \cdot \xi) ; \quad \xi \cdot \omega=\mathbf{0} .
$$

Note that in $\mathbf{R}^{3}$, the Radon transform is an integration of a function over planes whereas the X-ray transform is still an integration over lines. In addition, the projection direction $\omega$ is perpendicular to the Radon vector $\xi$. The properties of the Radon transform discussed in $§ 2.2 .2$ hold for the X-ray transform except the formulation of the Central Slice Theorem. The Central Slice Theorem is modified to reflect the change in definition of projection direction. In $\mathbf{R}^{3}$, it reads:

$$
\mathcal{F}_{3} f\left(\mathbf{k}_{x}\right)=\mathcal{F}_{2} \mathcal{P} f\left(\mathbf{k}_{u}, \omega\right)
$$

Comparing this to Eqn. 2.33, we see that the only difference between them is that the dimensionality of the Fourier transform performed on the right-hand-side. Proof of Eqn. 5.4 follows.

$$
\begin{aligned}
{\left[\mathcal{F}_{\mathbf{u}} \mathcal{P} f\right]\left(\mathbf{k}_{\mathbf{u}}, \omega\right) } & =\int d \mathbf{u} e^{-i \mathbf{k}_{\mathbf{u}} \cdot \mathbf{u}} \mathcal{P} f(\mathbf{u}, \omega) \\
& =\int d \mathbf{u} e^{-i \mathbf{k}_{\mathbf{u}} \cdot \mathbf{u}} \int d t f(\mathbf{u}-t \omega)
\end{aligned}
$$

Now recall that $\mathbf{u} \perp \omega$ so that $\mathbf{x}=\mathbf{u}-\omega t \Rightarrow d \mathbf{x}=d \mathbf{u} d t$. Therefore

$$
\begin{aligned}
{\left[\mathcal{F}_{\mathbf{u}} \mathcal{P} f\right]\left(\mathbf{k}_{\mathbf{u}}, \omega\right) } & =\left.\int d \mathbf{u} d t e^{-i \mathbf{k}_{\mathbf{u}} \cdot \mathbf{u}} e^{-i k_{t} t} f(\mathbf{x})\right|_{k_{t}=0} \\
& =\left.\mathcal{F}_{n} f\left(\mathbf{k}_{\mathbf{x}}\right)\right|_{k_{t}=0}
\end{aligned}
$$

It may seem that as in the case of the Radon transform in $2 \mathrm{D}$, reconstructing PET images in 3D means inverting the X-ray transform. Indeed, that would be the case if, as in the case of $2 \mathrm{D}$ reconstruction, we have a sampling of all the projections of the object we are reconstructing. Note that this may seem contradictory to an earlier statement that one does not need all the projections of a 3D image to reconstruct 
the object. To reconstruct a 3D object, one can stack $2 \mathrm{D}$ reconstructions of the object. But this is not what is meant by "fully 3D" reconstruction for which all the projections are needed. However, unless PET cameras cover the entire object being scanned, there will always be projections that are not sampled.

Hence, I will not give the inversion of the X-ray transform. Instead, I will discuss 3D image reconstruction that is based on the X-ray transform but does not explicitly involve its inversion, but rather, a 3D generalization of the 2D Filtered Backprojection procedure. An inversion of the X-ray transform using the Riesz Potential is given by Natterer [26].

\subsection{Filtered Backprojection in 3D and Colsher's Filter}

Recall the definition of the Backprojection operator $\mathcal{B}$ in $\mathbf{R}^{2}$ (Eqn. 2.39). Backprojection of the projection data in $\mathbf{R}^{2}$ resulted in the blurred image of the object. More precisely, given $f(\mathbf{x}), \mathbf{x} \in \mathbf{R}^{2}$ as the object in the FOV and $p(\mathbf{s})$ as the projection data,

$$
\mathcal{B} p(\mathbf{s})=f(\mathbf{x}) \otimes \frac{1}{\sqrt{x^{2}+y^{2}}} .
$$

In filtered backprojection techniques, the deconvolution is performed in the frequency space of the projection data before it is backprojected. Colsher [68] extended this method into $\mathbf{R}^{3}$ by determining the filter that needs to be applied to the $3 \mathrm{D}$ projection data before it is backprojected into the FOV. I detail his method below.

Colsher's method can be seen as a system and response problem where the signal is the object $f$, the system is the blurring function $h$ and the response is the reconstructed object $g$. We can write this as

$$
g(\mathbf{x})=\int d^{3} x^{\prime} f\left(\mathbf{x}^{\prime}\right) h\left(\mathbf{x}, \mathbf{x}^{\prime}\right) .
$$

If the response function $h$ is shift invariant, then we can write Eqn. 5.8 as

$$
g(\mathbf{x})=\int d^{3} x^{\prime} f\left(\mathbf{x}^{\prime}\right) h\left(\mathbf{x}-\mathbf{x}^{\prime}\right),
$$




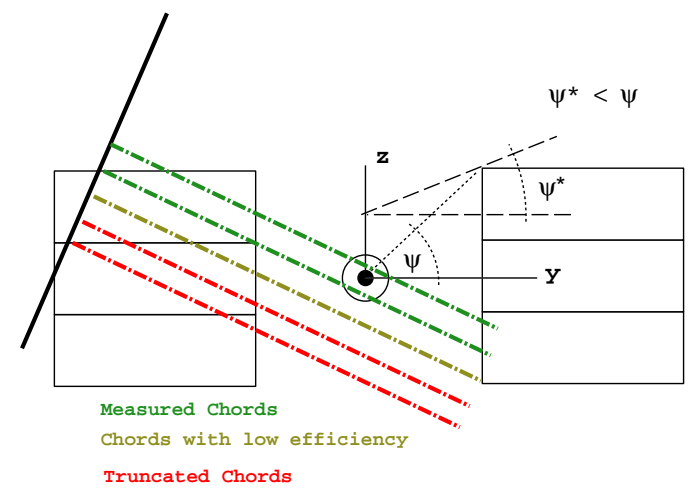

(A)

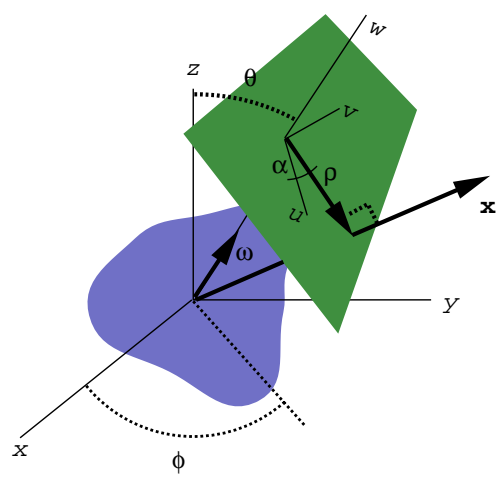

(B)

Figure 5.4: Definition of Colsher Polar acceptance angle $\psi(\mathrm{A})$ and variables $\rho$ and $\alpha$ in the Colsher filter (B). (A) also shows the truncation of projection data due finite extent of the camera in $z$. The green chords are measured while the red ones are not.

which can be written in frequency space as

$$
G(\mathbf{k})=F(\mathbf{k}) H(\mathbf{k})
$$

Thus, if $h$ is shift invariant, $f$ is obtained by the inverse Fourier transform of $G(\mathbf{k}) H(\mathbf{k})^{-1}$. Otherwise, the system response $h$ needs to be determined for each point within the FOV.

Given the largest "polar" angle accepted by the camera as $\theta_{\max }=\psi$, as shown in fig. 5.4(A), the response function at the center of the tomograph, in spherical coordinates, is given by [69]

$$
h(r, \theta, \phi)=\frac{1}{r^{2}} \Pi\left(\theta ; \frac{\pi}{2}, \psi\right)
$$

where $\Pi$ is the pulse function defined in Eqn 2.3. The factor $\frac{1}{r^{2}}$ comes from the dimensionality of the problem, i.e. the radial component of the volume element in $\mathbf{R}^{3}$ is $r^{2}$. The pulse function indicates that we will only backproject events which have a polar angle within $\psi$ of the $x-y$ plane. Note that for points on the $z$-axis, the value of $\psi$ varies, which means that the response function is not shift invariant. In 
order to satisfy the shift invariance constraint, conventional cameras take data from within a predefined extent in $z$ and the minimum $\psi^{*}$ is used. $\psi^{*}=0$ is equivalent to stacking $2 \mathrm{D}$ reconstructions to make up the $3 \mathrm{D}$ image.

I now give a derivation of Colsher's Filter, taken from [68]. Because of the azimuthal symmetry of $h(r, \theta, \phi)$, Colsher calculates $H\left(k_{r}, \Theta\right)$, the Fourier transform of $h(r, \theta)$ by using the following form of the transform [70]

$$
\begin{aligned}
H\left(k_{r}, \Theta\right) & =2 \pi \int_{0}^{\infty} r^{2} d r \int_{0}^{\pi} \sin \theta d \theta h(r, \theta) J_{0}\left(2 \pi k_{r} r \sin \Theta \sin \theta\right) \exp \left(-2 \pi i k_{r} r \cos \Theta \cos \theta\right) \\
& =\frac{\pi}{\psi} \int_{\frac{\pi}{2}-\psi}^{\frac{\pi}{2}+\psi} \sin \theta d \theta \int_{0}^{\infty} d r J_{0}\left(2 \pi k_{r} r \sin \Theta \sin \theta\right) \exp \left(-2 \pi i k_{r} r \cos \Theta \cos \theta\right) \\
& =\frac{1}{2 \psi k_{r}} \int_{\frac{\pi}{2}-\psi}^{\frac{\pi}{2}+\psi} \frac{\sin \theta d \theta}{\sqrt{\sin ^{2} \Theta \sin ^{2} \theta-\cos ^{2} \Theta \cos ^{2} \theta}}
\end{aligned}
$$

since $[71]$

$$
\int d t J_{0}(a t) e^{i b t}=\frac{1}{\sqrt{a^{2}-b^{2}}}
$$

$J_{0}$ is the $0^{t h}$ order Bessel Function. Using several trigonometric substitutions, Eqn. 5.12 can be reduced to

$$
\begin{aligned}
H\left(k_{r}, \Theta\right) & =\frac{|\sin \psi|}{2 \psi k_{r}} \int_{\mathbf{R}} d \sin \theta \frac{\Pi(\sin \theta ; 0, \sin \psi)}{\sqrt{\sin ^{2} \Theta-\sin ^{2} \theta}} \\
& =\frac{1}{2 \psi k_{r}}\left\{\arcsin \left(\frac{\sin \psi}{|\sin \Theta|}\right)-\arcsin \left(\frac{-\sin \psi}{|\sin \Theta|}\right)\right\}
\end{aligned}
$$

where the integration in the last step is done by parts. Recalling that the arcsin function is complex when $|\Theta| \leq \psi$, the reciprocal of $H\left(k_{r}, \Theta\right)$ may be written as

$$
H^{-1}\left(k_{r}, \Theta\right)= \begin{cases}\frac{\psi k_{r}}{\arcsin \frac{\sin \psi}{|\sin \Theta|}} & |\Theta|>\psi \\ \frac{2 \psi k_{r}}{\pi} & |\Theta| \leq \psi\end{cases}
$$

Note that in the limiting case that $\psi=0$, corresponding to a $2 \mathrm{D}$ scan, $H^{-1}$ reduces to $k_{r}|\sin \Theta|$ which is the projection of the vector $\mathbf{k}$ onto the $x y$ plane. This is the Ramp Filter discussed in $§ 2.2 .4$. 
The filter that is applied to the planes before they are backprojected are therefore given by the slices through this 3D filter,

$$
H_{\theta}^{-1}(\rho, \alpha)= \begin{cases}\frac{\psi \rho}{\sin ^{-1} \frac{\sin \psi}{\sqrt{\sin ^{2} \alpha+\cos ^{2} \alpha \sin ^{2} \theta}}} & \text { if }\left|\sin ^{2} \alpha+\cos ^{2} \alpha \sin ^{2} \theta\right|>\sin ^{2} \psi \\ \frac{2 \psi \rho}{\pi} & \text { otherwise }\end{cases}
$$

$(\rho, \alpha)$ are the cylindrical-polar coordinates within the projection plane. They are illustrated in fig. 5.4(B)

\subsubsection{Truncation: Missing Information}

The thrust behind the formulation of Colsher's Filter was the assumption that the blurring function of the camera is spatially invariant. Filtering the projection data prior to backprojection is equivalent to deconvolving the blurring function from the image, and this only works if the function has the form $h\left(\mathbf{x}, \mathbf{x}^{\prime}\right)=h\left(\mathbf{x}-\mathbf{x}^{\prime}\right)$. The spatial variance of the blurring function can be corrected by the appropriate normalization, just as we have done in the $2 \mathrm{D}$ case. Post-normalization, the corrected data approximates data taken from a system with a spatially invariant response function.

Colsher's method works if all projections within the polar range $\left|\theta-\frac{\pi}{2}\right|<\psi$. However, because PET cameras, do not cover the entire subject being scanned, some projection bins which are within the designated polar range are truncated, i.e. they are not measured. Fig. 5.4 shows this truncation. While normalization corrects for the variations in the efficiency of each projection bin, it cannot restore the truncated data. This truncation makes the response function spatially variant, with the truncated data effectively being zero efficiency bins. This missing information needs to be addressed before Colsher's filter can be applied to the data.

Fig. 5.5 shows the effect of truncation on the ideal projections of the PEM FOV shown in Fig. 5.3. Because of the shape of the PEM, the truncation is dependent on the azimuthal angle $\phi$. This is contrast to circular cameras where the truncation 

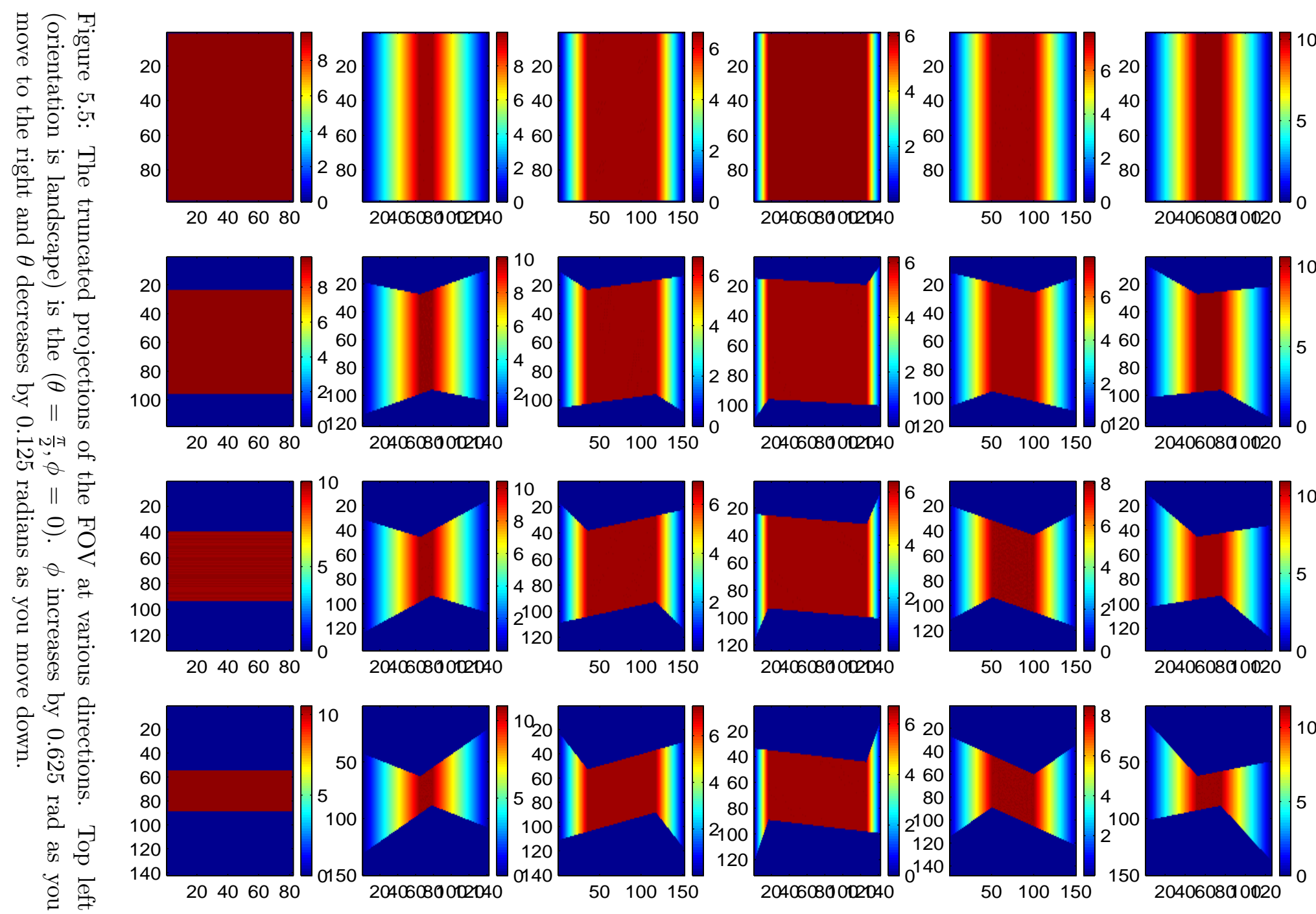


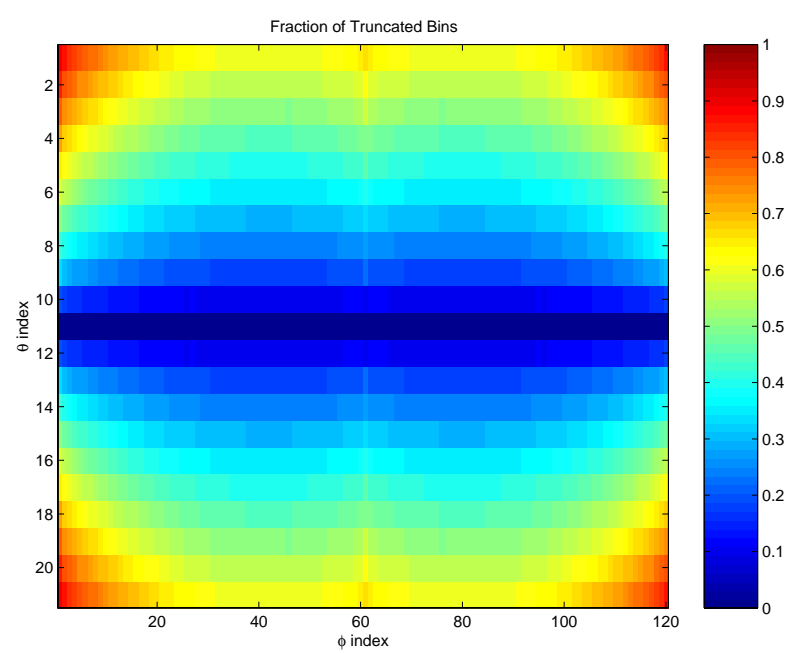

Figure 5.6: Fraction of truncated bins in each projection plane. $\theta$-index 11 corresponds to $\theta=\frac{\pi}{2}$ and each index corresponds to a projection that has a polar angle that is 0.05 radians from the previous one. $\phi$-index 60 is $\phi=\frac{\pi}{2}$ and each index corresponds to a projection that is 0.025 radians from the previous one.

depends only on the polar angle $\theta$. Fig. 5.6 shows the fraction of bins in each projection plane that are truncated.

\subsubsection{Kinihan and Rogers Algorithm (3DRP)}

The solution for the truncation problem lies in the redundancy of 3D projection data. As I noted earlier, while the object being scanned is a function of three variables, $\mathbf{x} \in \mathbf{R}^{3}$, the projection data is a function of four variables, $\mathbf{s} \in \mathbf{R}^{2} \otimes \boldsymbol{\Omega}_{2}$. Kinihan and Rogers exploited this fact to resolve the truncation problem with their 3D Reprojection-Projection algorithm (3DRP) [72]. Their method is akin to the IRR algorithm of Medoff et al. discussed in $\S$ 4.3.2. An initial 3D image is reconstructed from stacks of $2 \mathrm{D}$ images taken from the in-plane data. This is analogous to reconstructing an initial image with the incomplete data in the IRR algorithm. The difference between the two procedures is that while the initial image in the IRR 
algorithm is only an approximate image, even if there were an infinite number of events, the initial 3D image in the 3DRP is in fact a true representation of the image, differing only from a full $3 \mathrm{D}$ reconstruction by its noise properties. Therefore, if there were an infinite number of in-plane events, there would be no need to perform $3 \mathrm{D}$ reconstruction. However, such is not the case.

The 3DRP algorithm then forward projects the initial 3D image into the truncated projections, again in a very similar fashion as in the IRR algorithm. The difference this time is that while a priori information is needed in the IRR algorithm, there is no such requirement in the 3DRP algorithm. Again, this is because the stack of $2 \mathrm{D}$ images is a true representation of the $3 \mathrm{D}$ image. Finally, while the IRR may need several iterations of this procedure, the 3DRP only requires one.

Thus, the 3DRP algorithm is a two-pass algorithm using Colsher's filter, once with $\psi=0$ and another with $\psi=\psi^{*}$. This has the benefit of increasing the number of events that are used in the reconstruction procedure. In their original work, Kinihan and Rogers essentially quadrupled the number of useful events in their reconstruction by using $\psi^{*}=0.1047$ radians. As I shall show, this procedure increases the useful events in the PEM camera by factor of 20 by using $\psi^{*}=0.5375$ radians.

The large acceptance angle of the PEM camera is the primary reason behind the necessity of using 3DRP. There are many other algorithms that incorporate off-plane events in the reconstruction. However, most of these algorithms are approximations requiring that the polar acceptance of the detector be small. Among these are the Single Slice Rebinning Algorithm (SSRA) [73] and the Multi Slice Rebinning Algorithm (MSRA) [74].Both rebin the off-plane events into in-plane projections and then reconstruct the 3D image by stacking $2 \mathrm{D}$ images. The SSRA rebins an event to the plane at the mid point of the chord while the MSRA distributes an event along all the planes that the chord intersects. The performances of these algorithms depend greatly on a small acceptance angle.

A more recent technique for $3 \mathrm{D}$ reconstruction is the Fourier Rebinning 
Algorithm (FORE) [75]. Defrise et al. performs the rebinning of the off-plane events in Fourier space instead of object space, and in the process, relaxing the requirement of small acceptance angles. At the time when I began studying reconstruction algorithms for the PEM camera, the implementation of the FORE was only approximate. The approximation required was $\psi^{*}<0.25$ or about half the acceptance of the PEM camera. More recently, Xuam et al. [76] have developed implementations of the FORE algorithm and have corrected the image distortions generated by the FORE algorithm at large polar angles. The new algorithm, called FOREX, is capable of reconstructing images from cameras with polar acceptances of $\frac{\pi}{6}$, comparable to that of the PEM camera's. I discuss the theory behind FOREX in Appendix A because this may be a possible future direction for reconstructions for the PEM camera. However, the idiosyncrasies of the PEM camera would undoubtedly require the modification of the FOREX algorithm if it were used.

\subsection{Normalization in $\mathbf{R}^{3}$}

As in the $2 \mathrm{D}$ reconstruction, the data needs to be normalized if filtered backprojection is used. $\operatorname{In} \mathbf{R}^{3}$, the solid angle subtended by each detector pair varies considerably. In particular, the detector pair close to the corners of the detector subtends a much larger solid angle than a crystal pair on opposite banks.

In order to get the normalization data, we compare a flood phantom to the line integrals through a rectangular parallelepiped that has the dimensions of the FOV. The path of the line integrals depends on the parameters that will be used for the projection data. However, in order to determine the optimal parameters for the projection data, we need a normalization set in order to use filtered backprojection. Thus, in anticipation of the optimal parameters, I will use the parameters determined in the later sections to discuss the normalization set.

Recall that from $\S 4.2$, the normalization set is calculated on a projectionbin-by-projection-bin basis by dividing the calculated line integral with the number 

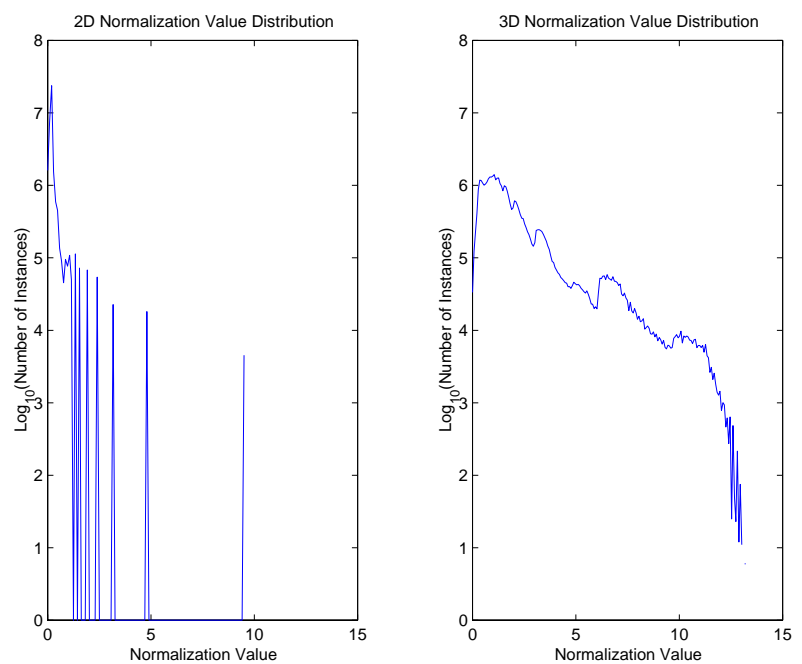

Figure 5.7: Distributions of Normalization values, for 2D and 3D. The 2D figure has been normalized so that it has the same number of events as the 3D histogram.

of events detected in that particular projection bin. The same procedure is used here. However, there are two main differences between the normalization in the $\mathbf{R}^{2}$ and the present one. First, because of the truncation, there will be many more zero-efficiency bins for the $\mathbf{R}^{3}$ normalization data, although unlike the $\mathbf{R}^{2}$ case where the lack of sampling is due to the binning of the data, all of these bins are truly unsampled. Fig. 5.5 shows the truncation of the projections of the FOV. Second, because of the increase in the dimensionality of the problem and the drop in geometrical efficiency in going from $2 \mathrm{D}$ to $3 \mathrm{D}$, the number of events necessary to generate a normalization file is far greater than the $10^{7}$ events used for the normalization in $2 \mathrm{D}$. The plots that will follow come from data sets with $8 \times 10^{8}$ annihilations.

Because of the finite number of events in the simulated flood phantom, I need to put a threshold on the normalization value that I will use, setting those above this threshold to 0 . In $\mathbf{R}^{2}$, the distribution of normalization values is such that a cut may be easily determined by observation. Fig. 5.7 shows the distribution of $2 \mathrm{D}$ and 
$3 \mathrm{D}$ normalization values. The $2 \mathrm{D}$ histogram has been normalized to the number of events in the $3 \mathrm{D}$ histogram. Note that a value such as 1.5 can be easily justified as the cutoff for the $\mathbf{R}^{2}$ normalization values just by looking at the plot. This is not the case for the $3 \mathrm{D}$ histogram. The reason is that in $2 \mathrm{D}$, once a threshold normalization is determined, I can check the relative number of bins that are going to have zero efficiency by simply looking at the $2 \mathrm{D}$ sinogram of the normalization values. Ideally, the normalization threshold is such that it minimizes the number of zero-efficiency bins. In $\mathbf{R}^{3}$, it is conceivable that a threshold may tag a large number of bins as zero- efficiency bins at particular projection directions. There is no guarantee that the fraction of bins set to zero is not all located in a few arbitrary directions.

In order to choose the efficiency cut-off for the $3 \mathrm{D}$ reconstruction, I look at the fraction of bins that are labeled as zero efficiency bins as a function of the normalization cut and of the direction. I use $\theta$ and $\phi$ to parameterize the projection direction. Fig. 5.8 shows this plot for cutoffs of $e_{T}=(1,3,5,7,9,11)$. The plots display the fraction of bins in each projection direction that is excluded, i.e. labeled as zero-efficiency bins. Fig. 5.9 shows the distribution of the fraction of bins labeled as zero-efficiency bins. From these plots, it appears that the cut $e_{T}=6$ will do. This means that averaged over the projection directions, $20 \%$ of the bins will be labeled as zero-efficiency bins.

\subsection{Polar Acceptance}

One of the important parameters in 3D reconstruction is the polar acceptance of the camera. Cameras with large polar-angle acceptance have the advantage of increased sensitivity and thus better statistics and better reconstructed image quality. However, large polar acceptance can also mean large data sets which may pose problems in data storage and in image reconstruction. Reconstruction algorithms scale at best linearly with the size of the data set.

Determining how much of the available polar angle acceptance to use is an 


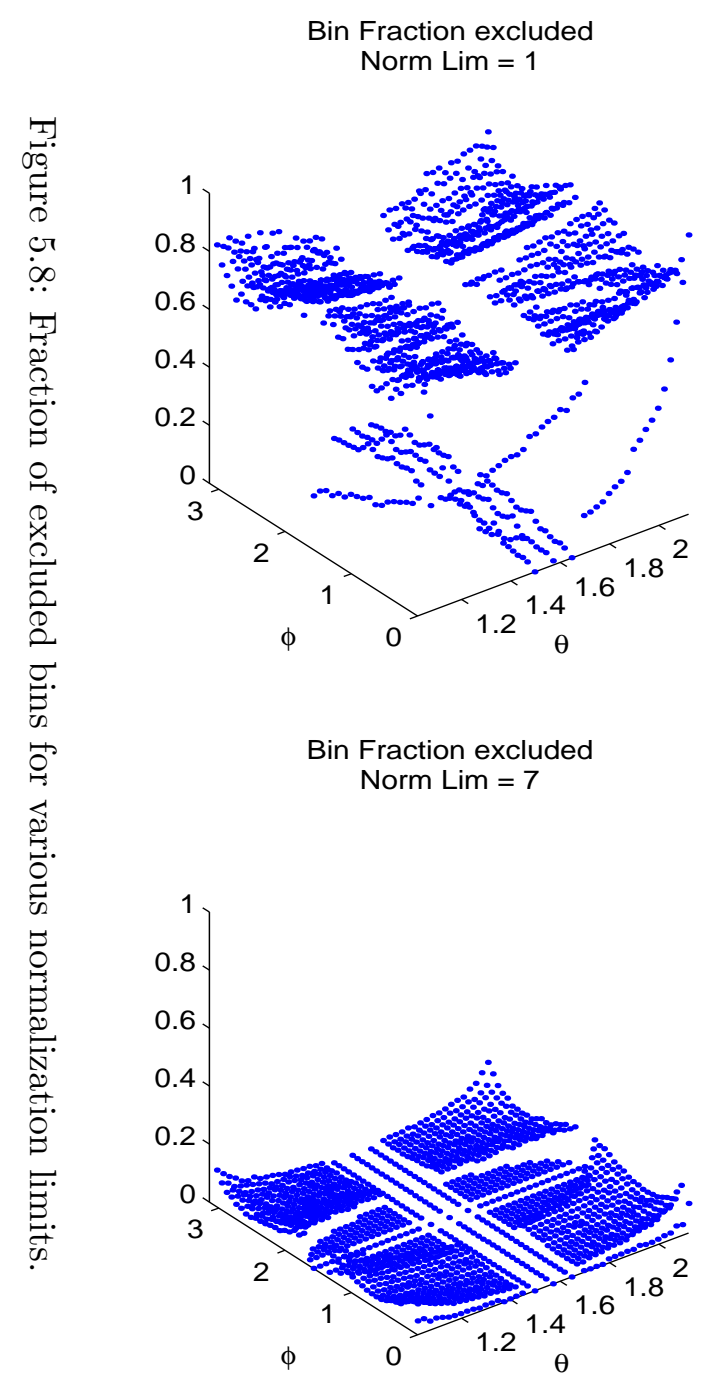

Bin Fraction excluded

Norm Lim $=3$

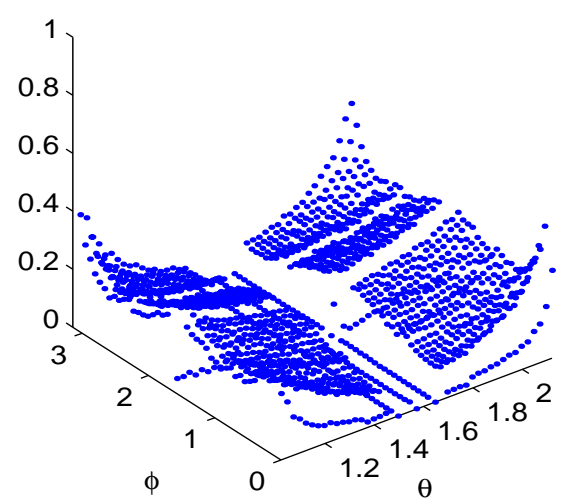

Bin Fraction excluded

Norm Lim $=9$

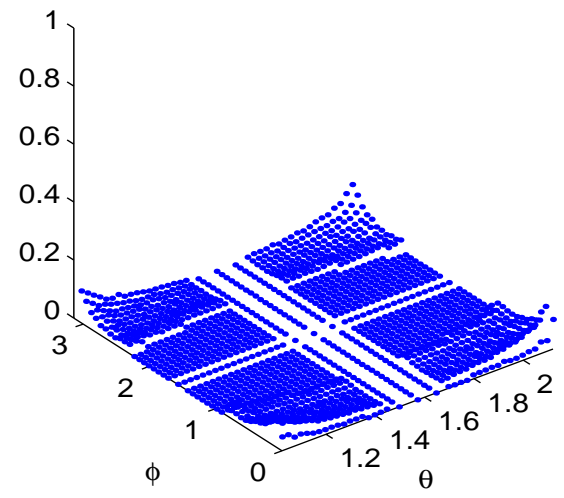

Bin Fraction excluded

Norm Lim $=5$

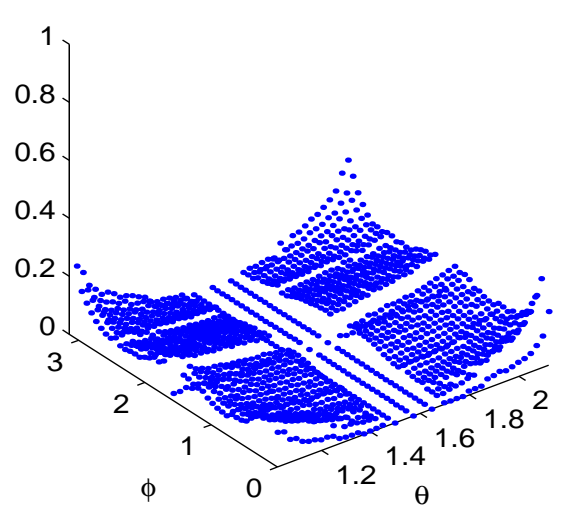

Bin Fraction excluded

Norm Lim $=11$

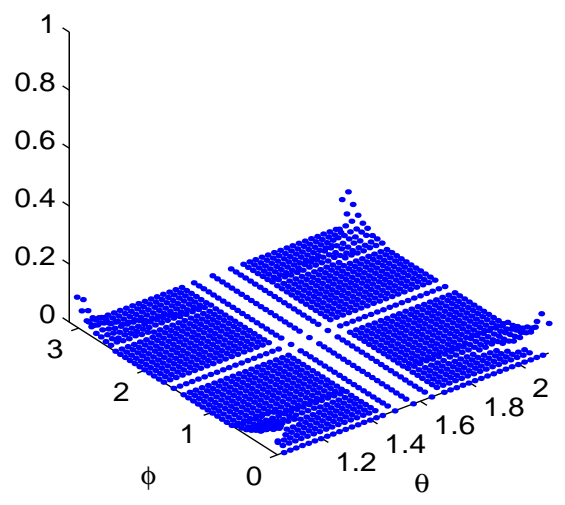



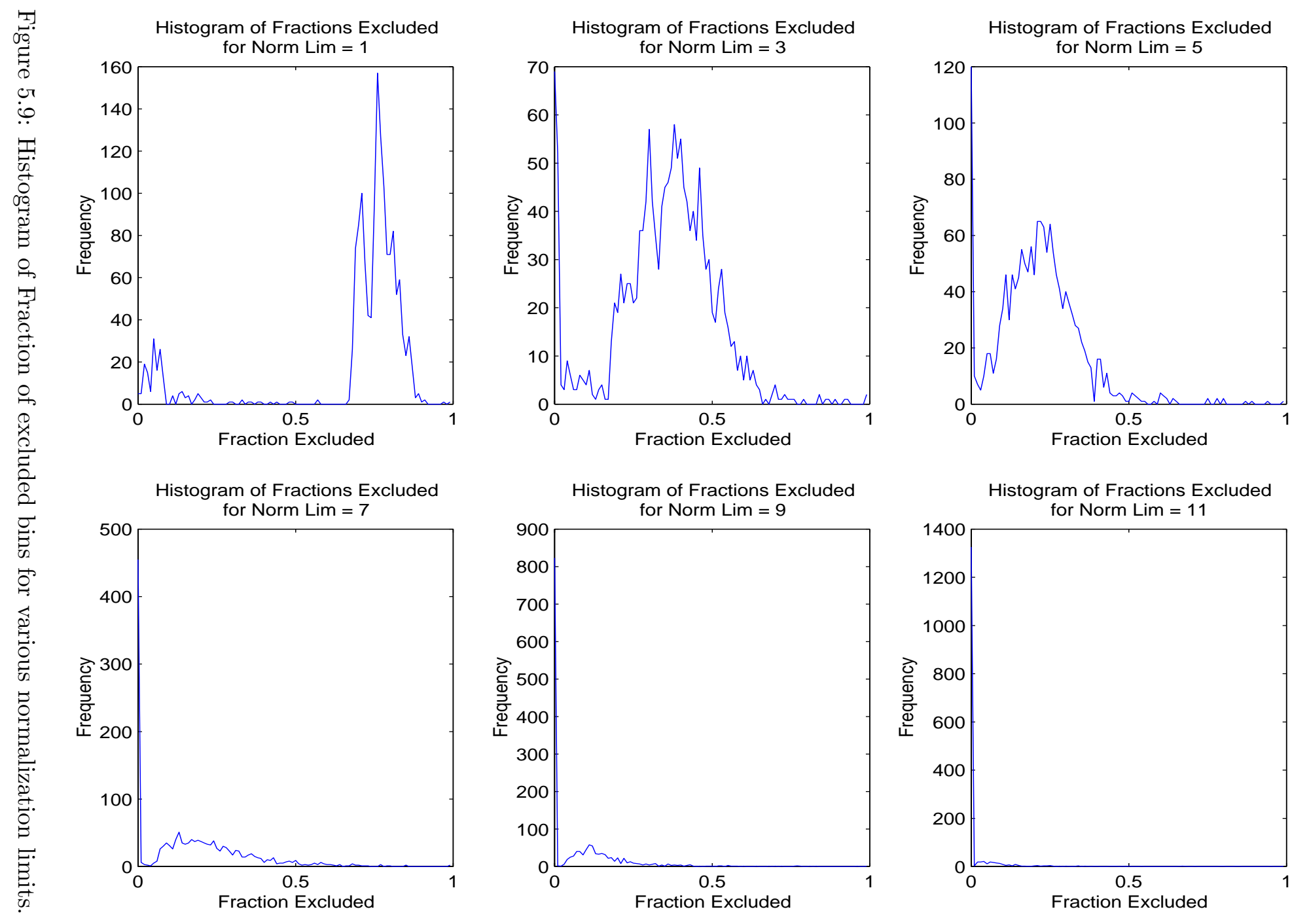

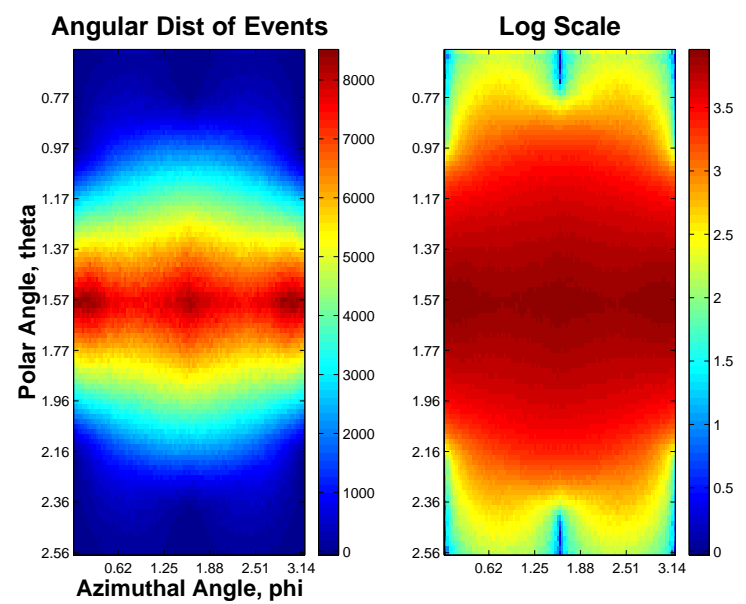

Figure 5.10: Angular Distribution of Detected Events.

optimization that I need to do. Recall that the solid angle available as a function of $\theta$ is $\sin \theta$ and thus the fraction of events that falls between $\theta$ and $\theta+d \theta$ is monotonically decreasing as $\theta$ decreases from $\frac{\pi}{2}$. This means that at some $\theta$, including more solid angle increases our data size without the payoff of actually getting significantly more events. Fig 5.10A plots the angular distribution of events detected by the PEM camera. This histogram is taken from the true interaction positions of two photons from a flood source in the field of view, i.e. the positions are not moved to lie along the main axis of the crystals. Fig $5.10 \mathrm{~B}$ is the same plot in $\log _{10}$ scale. The decrease in the population of events around $\phi=.90$ and $\phi=2.5$ are the familiar regions where the detector banks overlap.

A large fraction of the events are contained along the band $\left|\theta-\frac{\pi}{2}\right|<.2$. However, the average number of events does not drop by an order of magnitude until $\left|\theta-\frac{\pi}{2}\right|<.6$. Fig 5.11 plots fraction of events that fall between $\frac{\pi}{2}$ and $\theta$ as a function of $\theta$. $90 \%$ of events are within 0.38 of $\theta=\frac{\pi}{2}$. Beyond this, in order to determine how much of the available acceptance we need to use, we have to reconstruct images using varying acceptances and evaluate how the images compare with each other. 


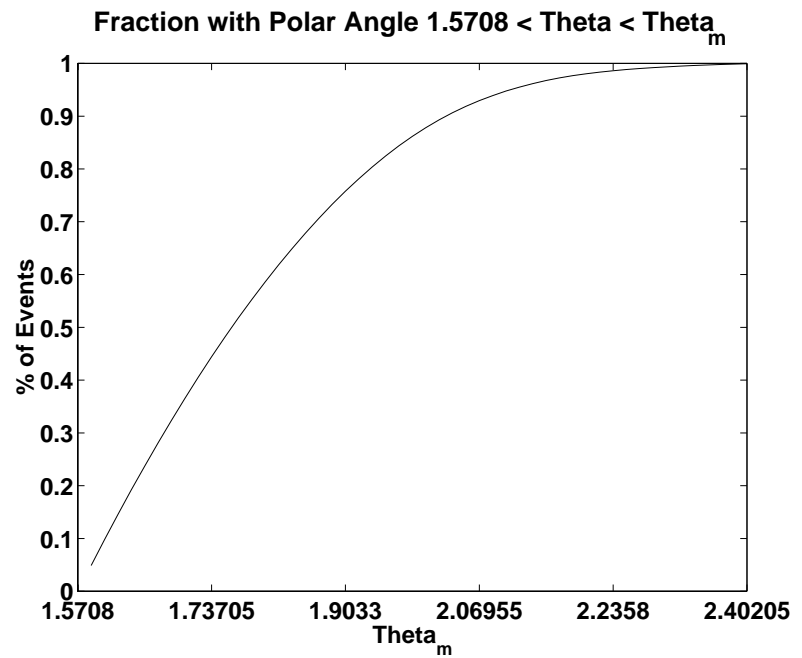

Figure 5.11: Cumulative Polar Distribution of Detected Events.

\subsubsection{Signal-to-Noise Measurements: Parameters and Algorithms}

Since the primary benefit of 3D imaging is noise reduction, I can use signalto-noise ratios as a figure of merit to determine how much polar acceptance to use. For this purpose, I simulate a flood phantom and calculate the mean and variance of pixels at various locations of the reconstructed image as a function of the polar acceptance. Within the region of interest, the mean and variance estimates signal and noise, respectively.

The data is taken from a simulation of the ideal 3D detector. The interaction locations are discretized and are initially assigned to the centers of the DOI bin as I have done previously. The interaction point is then allowed to take a random value within the DOI bin and the new location is used in the calculation of the parameters of the event. The data are a simulation of $10^{8}$ annihilations. The flood source from which the normalization is calculated is eight times this number.

I use $\frac{d}{2}$ sampling so that I need not worry about the complications of unsampled projections other than those which are the result of data truncation. I use 


\begin{tabular}{cccccc}
\hline \hline Proj Num & $\theta$ & $\delta \theta$ & Proj Num & $\theta$ & $\delta \theta$ \\
\hline 0 & 0.770796 & 0.050 & 17 & 1.620796 & 0.075 \\
\hline 1 & 0.820796 & 0.050 & 18 & 1.670796 & 0.050 \\
\hline 2 & 0.870796 & 0.050 & 19 & 1.720796 & 0.050 \\
\hline 3 & 0.920796 & 0.050 & 20 & 1.770796 & 0.050 \\
\hline 4 & 0.970796 & 0.050 & 21 & 1.820796 & 0.050 \\
\hline 5 & 1.020796 & 0.050 & 22 & 1.870796 & 0.050 \\
\hline 6 & 1.070796 & 0.050 & 23 & 1.920796 & 0.050 \\
\hline 7 & 1.120796 & 0.050 & 24 & 1.970796 & 0.050 \\
\hline 8 & 1.170796 & 0.050 & 25 & 2.020796 & 0.050 \\
\hline 9 & 1.220796 & 0.050 & 26 & 2.070796 & 0.050 \\
\hline 10 & 1.270796 & 0.050 & 27 & 2.120796 & 0.050 \\
\hline 11 & 1.320796 & 0.050 & 28 & 2.170796 & 0.050 \\
\hline 12 & 1.370796 & 0.050 & 29 & 2.220796 & 0.050 \\
\hline 13 & 1.420796 & 0.050 & 30 & 2.270796 & 0.050 \\
\hline 14 & 1.470796 & 0.050 & 31 & 2.320796 & 0.050 \\
\hline 15 & 1.520796 & 0.075 & 32 & 2.370796 & 0.050 \\
\hline 16 & 1.570796 & 0.075 & & & \\
\hline
\end{tabular}

Table 5.2: Polar projection angles and widths. 
$\delta \theta=0.05$ for all of the polar projection angles, except at $\theta=\frac{\pi}{2}$ and the projection angle on either side where I use $\delta \theta=0.075$. The reason for using this specific value becomes clear when you consider that from the origin, the largest arc length between two crystal centers in the polar direction is along the $y$-axis perpendicular to the $x y$ plane. This arc length is the same as in the azimuthal direction where $\delta \phi=0.075$. Table 5.2 lists the polar angles of projection and their widths.

The image is divided into cubic voxels that have a linear dimension of $\frac{d}{2}=.15 \mathrm{~mm}$, equal to that of the projection bins. This means that there are a total of $N_{x}=64, N_{y}=40$ and $N_{\text {plane }}=48$. The numbering of the planes is such that plane 0 is defined to be at the most negative $z$.

At first attempt, I use the FW angular scheme determined in our 2D reconstruction exercise, but with the angular widths twice the nominal value except at $\phi=0, \frac{\pi}{2}$ where $\delta \phi_{0}=0.05$ and $\delta \phi_{\frac{\pi}{2}}=.075$. Therefore, I use $N_{\phi}=6+\frac{120-6}{2}=63$. Recall that there is a $\frac{\pi}{2}$ difference in the definition of $\phi$ in $\mathbf{R}^{\mathbf{2}}$ and $\mathbf{R}^{\mathbf{3}}$ reconstruction so that the width $\delta \phi=0.075$ is associated with the $\phi=\frac{\pi}{2}$ direction in $\mathbf{R}^{3}$. The reason for using twice the nominal width is that it seems reasonable to assume that since we are using $\frac{d}{2}$ linear sampling instead of $\frac{d}{4}$, sampling the azimuthal angle at half the rate would be sufficient. However, the resulting reconstructed image of a flood phantom reveals the insufficiency of sampling $\phi$ at a lower rate. Streaking in the image was severe. Thus, the azimuthal angle will always be sampled at the angles prescribed by the FW angular scheme determined in our initial $2 \mathrm{D}$ reconstruction. The values are given in Tables 5.3 and 5.4.

The reconstruction uses the standard 3DRP algorithm [68] modified to accommodate the irregular angular sampling both in $\theta$ and $\phi$. The standard algorithm is discussed in $§ 5.2 .2$. In general, the modifications to the standard 3DRP algorithm begins with the low-statistics $2 \mathrm{D}$ image being reconstructed in the manner discussed in Chapter $\S 4$. The resulting image is forward projected in the manner prescribed by the 3DRP algorithm. There is another modification to the algorithm at this point. The polar directions where $\delta \theta$ is larger than the nominal value $\delta \theta_{\text {nom }}$ is reprojected 


\begin{tabular}{cccccc}
\hline \hline Proj Num & $\phi$ & $\delta \phi$ & Proj Num & $\phi$ & $\delta \phi$ \\
\hline 0 & 0.025000 & 0.050000 & 30 & 0.778782 & 0.024268 \\
\hline 1 & 0.075000 & 0.050000 & 31 & 0.803050 & 0.024268 \\
\hline 2 & 0.099268 & 0.024268 & 32 & 0.827319 & 0.024268 \\
\hline 3 & 0.123536 & 0.024268 & 33 & 0.851587 & 0.024268 \\
\hline 4 & 0.147805 & 0.024268 & 34 & 0.875855 & 0.024268 \\
\hline 5 & 0.172073 & 0.024268 & 35 & 0.900124 & 0.024268 \\
\hline 6 & 0.196341 & 0.024268 & 36 & 0.924392 & 0.024268 \\
\hline 7 & 0.220610 & 0.024268 & 37 & 0.948660 & 0.024268 \\
\hline 8 & 0.244878 & 0.024268 & 38 & 0.972929 & 0.024268 \\
\hline 9 & 0.269146 & 0.024268 & 39 & 0.997197 & 0.024268 \\
\hline 10 & 0.293415 & 0.024268 & 40 & 1.021465 & 0.024268 \\
\hline 11 & 0.317683 & 0.024268 & 41 & 1.045734 & 0.024268 \\
\hline 12 & 0.341951 & 0.024268 & 42 & 1.070002 & 0.024268 \\
\hline 13 & 0.366220 & 0.024268 & 43 & 1.094270 & 0.024268 \\
\hline 14 & 0.390488 & 0.024268 & 44 & 1.118539 & 0.024268 \\
\hline 15 & 0.414756 & 0.024268 & 45 & 1.142807 & 0.024268 \\
\hline 16 & 0.439025 & 0.024268 & 46 & 1.167076 & 0.024268 \\
\hline 17 & 0.463293 & 0.024268 & 47 & 1.191344 & 0.024268 \\
\hline 18 & 0.487562 & 0.024268 & 48 & 1.215612 & 0.024268 \\
\hline 19 & 0.511830 & 0.024268 & 49 & 1.239881 & 0.024268 \\
\hline 20 & 0.536098 & 0.024268 & 50 & 1.264149 & 0.024268 \\
\hline 21 & 0.560367 & 0.024268 & 51 & 1.288417 & 0.024268 \\
\hline 22 & 0.584635 & 0.024268 & 52 & 1.312686 & 0.024268 \\
\hline 23 & 0.608903 & 0.024268 & 53 & 1.336954 & 0.024268 \\
\hline 24 & 0.633172 & 0.024268 & 54 & 1.361222 & 0.024268 \\
\hline 25 & 0.657440 & 0.024268 & 55 & 1.385491 & 0.024268 \\
\hline 26 & 0.681708 & 0.024268 & 56 & 1.409759 & 0.024268 \\
\hline 27 & 0.705977 & 0.024268 & 57 & 1.434027 & 0.024268 \\
\hline 28 & 0.730245 & 0.024268 & 58 & 1.458296 & 0.024268 \\
\hline 29 & 0.754513 & 0.024268 & 59 & 1.533296 & 0.075000 \\
\hline & & & & & \\
\hline
\end{tabular}

Table 5.3: A. Azimuthal projection angles and widths. 


\begin{tabular}{cccccc}
\hline \hline Proj Num & $\phi$ & $\delta \phi$ & Proj Num & $\phi$ & $\delta \phi$ \\
\hline 60 & 1.608296 & 0.075000 & 90 & 2.387078 & 0.024268 \\
\hline 61 & 1.683296 & 0.075000 & 91 & 2.411347 & 0.024268 \\
\hline 62 & 1.707564 & 0.024268 & 92 & 2.435615 & 0.024268 \\
\hline 63 & 1.731833 & 0.024268 & 93 & 2.459883 & 0.024268 \\
\hline 64 & 1.756101 & 0.024268 & 94 & 2.484152 & 0.024268 \\
\hline 65 & 1.780369 & 0.024268 & 95 & 2.508420 & 0.024268 \\
\hline 66 & 1.804638 & 0.024268 & 96 & 2.532688 & 0.024268 \\
\hline 67 & 1.828906 & 0.024268 & 97 & 2.556957 & 0.024268 \\
\hline 68 & 1.853174 & 0.024268 & 98 & 2.581225 & 0.024268 \\
\hline 69 & 1.877443 & 0.024268 & 99 & 2.605493 & 0.024268 \\
\hline 70 & 1.901711 & 0.024268 & 90 & 2.629762 & 0.024268 \\
\hline 71 & 1.925979 & 0.024268 & 101 & 2.654030 & 0.024268 \\
\hline 72 & 1.950248 & 0.024268 & 102 & 2.678298 & 0.024268 \\
\hline 73 & 1.974516 & 0.024268 & 103 & 2.702567 & 0.024268 \\
\hline 74 & 1.998784 & 0.024268 & 104 & 2.726835 & 0.024268 \\
\hline 75 & 2.023053 & 0.024268 & 105 & 2.751104 & 0.024268 \\
\hline 76 & 2.047321 & 0.024268 & 106 & 2.775372 & 0.024268 \\
\hline 77 & 2.071590 & 0.024268 & 107 & 2.799640 & 0.024268 \\
\hline 78 & 2.095858 & 0.024268 & 108 & 2.823909 & 0.024268 \\
\hline 79 & 2.120126 & 0.024268 & 109 & 2.848177 & 0.024268 \\
\hline 80 & 2.144395 & 0.024268 & 110 & 2.872445 & 0.024268 \\
\hline 81 & 2.168663 & 0.024268 & 111 & 2.896714 & 0.024268 \\
\hline 82 & 2.192931 & 0.024268 & 112 & 2.920982 & 0.024268 \\
\hline 83 & 2.217200 & 0.024268 & 113 & 2.945250 & 0.024268 \\
\hline 84 & 2.241468 & 0.024268 & 114 & 2.969519 & 0.024268 \\
\hline 85 & 2.265736 & 0.024268 & 115 & 2.993787 & 0.024268 \\
\hline 86 & 2.290005 & 0.024268 & 116 & 3.018055 & 0.024268 \\
\hline 87 & 2.314273 & 0.024268 & 117 & 3.042324 & 0.024268 \\
\hline 88 & 2.338541 & 0.024268 & 118 & 3.066592 & 0.024268 \\
\hline 89 & 2.362810 & 0.024268 & 119 & 3.116592 & 0.050000 \\
\hline & & & & & \\
\hline
\end{tabular}

Table 5.4: B. Azimuthal projection angles and widths. 


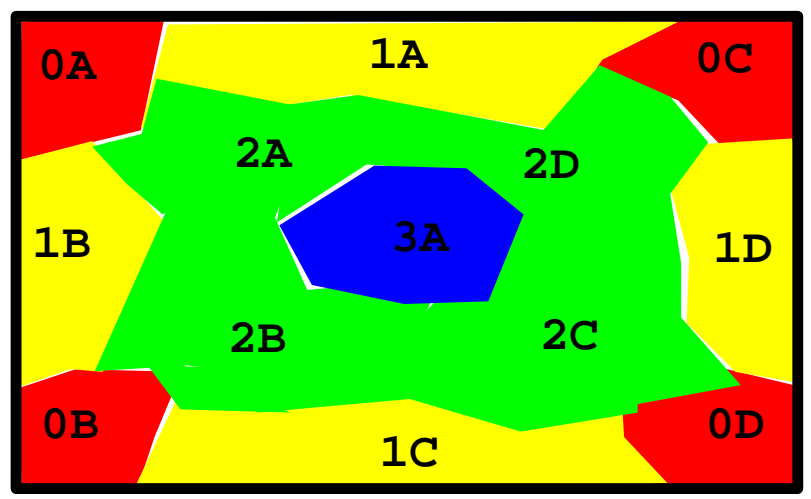

Figure 5.12: Regions in the FOV. (0) The corners (1) the edges (2) the bulk (3) the central region. The labels are used to identify where in each region the data are taken for the signal-to-noise measurements discussed in the text. The boundaries in the regions are meant to convey proximities of the defined areas.

$N_{\text {reproj }}(\theta) \in \mathbf{Z}$ times such that

$$
\delta \theta^{\prime}=\frac{\delta \theta}{N_{\text {reproj }}(\theta)}<\delta \theta_{\text {nom }} .
$$

This is so that an independent view of the object is used for the subsequent filtered backprojection. Thus, in the present case, the low-statistics $2 \mathrm{D}$ image is forward projected into the polar angles $\theta=\frac{\pi}{2} \pm 0.0375$ instead of at $\theta=\frac{\pi}{2}$. This means that we have two "independent" views to backproject during the final 3D filtered backprojection. Note that this is in contrast to the procedure outlined in $\S 4$ where the same view of the object is backprojected several times to address the issue of not having enough samples. This is one of the benefits of reconstructing in $\mathbf{R}^{3}$, i.e. because of the redundancy of the data, we can use the low-statistic image to estimate the data in the views that are not measured. The technique uses the same principle of estimating the truncated data with projections from the initial $2 \mathrm{D}$ reconstruction of the object.

Because the PEM camera does not have the full rotational symmetry that conventional PET cameras possess, I expect that the signal-to-noise ratio of the 
image will depend on where it is calculated in the field of view. For the purpose of identifying regions in the FOV, I divide the FOV into four regions: 0) the corner regions, 1) the edge regions 2) the bulk, and 3) the central region. The locations of these regions are indicated graphically in Fig. 5.12. The corners are unique in that the line-integral approximation of the data is bad because the crystal widths are comparable to the typical lengths in the region. Since the solid angle available for detecting photons is defined by the farthest detector, the edge regions are regions with the smallest solid angles and therefore the fraction of annihilations coming from this region is less than from other regions. The central region is unique because it is the region that is sampled the most.

To calculate the signal and the noise, we choose regions of interest in one of the four regions in the FOV. Choosing a region of interest involves defining a set of pixels for which the mean and the variance of the pixel values are calculated. To see the effect of varying ROI size, I perform the calculation for a $5 \times 5,10 \times 10$ and $15 \times 15$ pixel square region for each of the reconstruction planes.

\subsubsection{Signal-to-Noise Measurements: Results}

The results of the $\mathrm{S} / \mathrm{N}$ calculations are shown in figures 5.13 through 5.16. The ordinate shows the signal-to-noise ratio while the abscissa represents the number of $\theta$-projection sets used in the reconstruction. Thus, an increment in the $x$ axis represents the addition of another projection set in the reconstruction as compared to the previous reconstruction. The new set of projections is $\delta \theta=0.05$ radians from the previous one. Thus, the $2 \mathrm{D}$ reconstruction $\mathrm{S} / \mathrm{N}$ value is the data point at $x=1$, the reconstruction that involves the in-plane events and the events binned at

$\theta=\frac{\pi}{2}+.05$ is the data point at $x=2$, the reconstruction that involves the in-plane events plus the events binned at $\theta=\frac{\pi}{2}+.05$ and $\theta=\frac{\pi}{2}+.10$ is at $x=3$, and so on. The data point at $x=16$ represents the reconstruction that includes all the events.

Figures 5.13 through 5.15 are the plots for ROI's of sizes $5 \times 5,10 \times 10$ and 

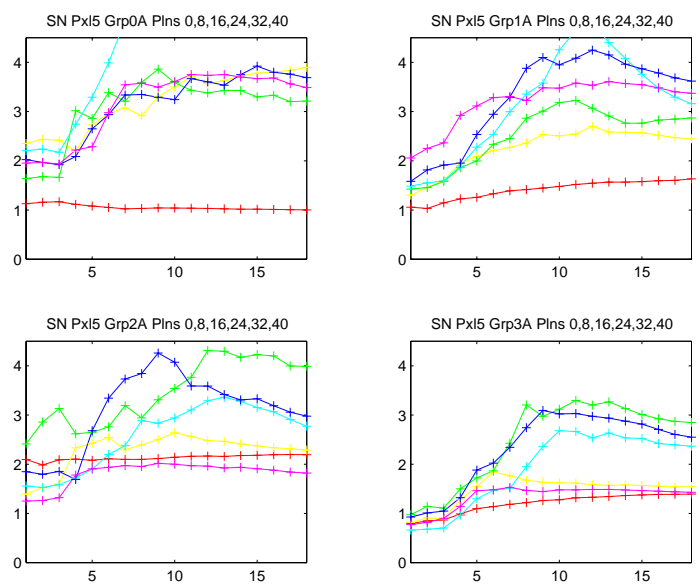

Figure 5.13: Signal-to-noise ratio of a $5 \times 5$ pixel array centered at $0 \mathrm{~A}, 1 \mathrm{~A}, 2 \mathrm{~A}$, and $3 \mathrm{~A}$ (Fig. 5.12) clockwise from the top left. Shown are planes 0(red), 8(yellow), 16(green), 24(cyan), 32(blue) and 40(purple). Each point along the abscissa represents the addition of another projection that is $0.05 \mathrm{rad}$ from the previous one. $x=0$ is the in-plane $2 \mathrm{D}$ reconstruction.
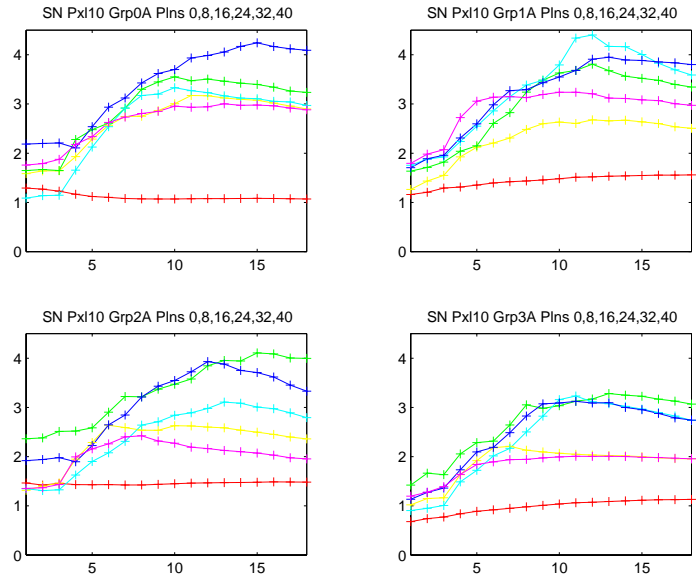

Figure 5.14: Signal-to-noise ratio of a $10 \times 10$ pixel array centered at $0 \mathrm{~A}, 1 \mathrm{~A}, 2 \mathrm{~A}$ and 3A (Fig. 5.12), clockwise from the top left. Shown are planes 0, 8, 16, 24, 32, 40. 

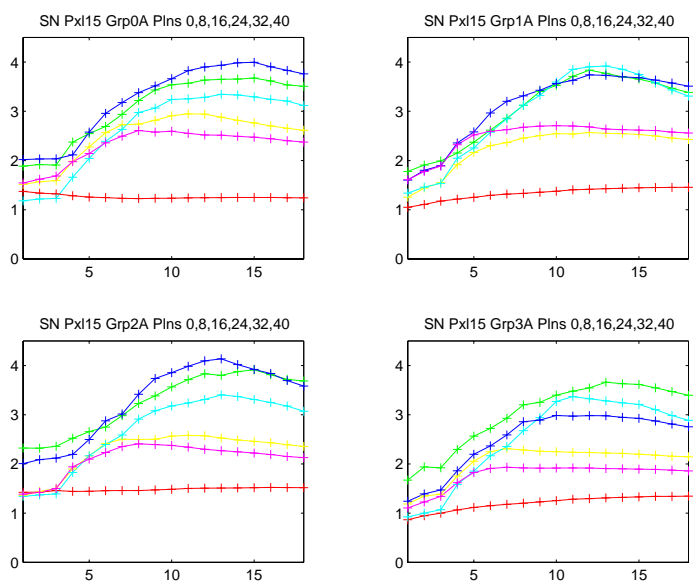

Figure 5.15: Signal-to-noise ratio of a $15 \times 15$ pixel array centered at $0 \mathrm{~A}, 1 \mathrm{~A}, 2 \mathrm{~A}$ and 3A (Fig. 5.12), clockwise from the top left. Shown are planes 0, 8, 16, 24, 32, 40.
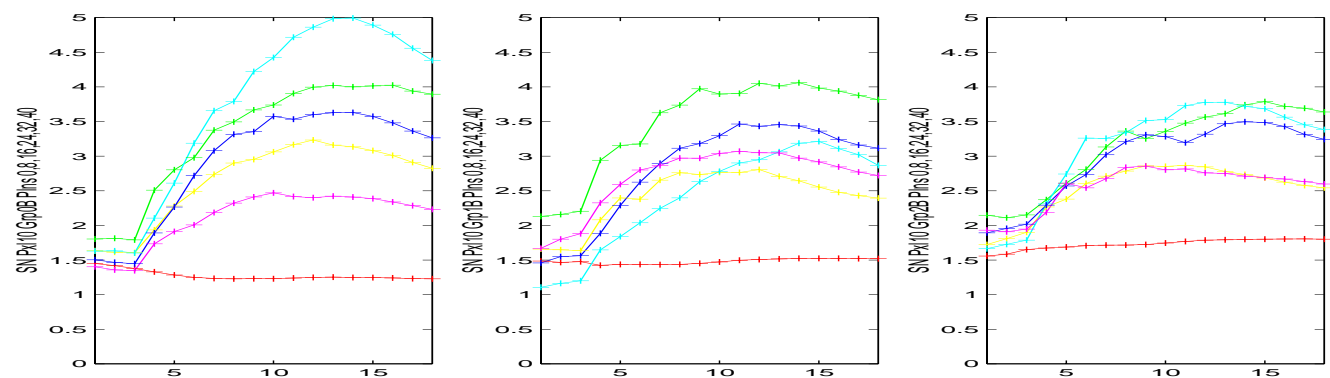

Figure 5.16: Signal-to-noise ratio of a $15 \times 15$ pixel array centered at $0 \mathrm{~B}, 1 \mathrm{~B}$ and $2 \mathrm{~B}$ from the left (Fig. 5.12). Shown are planes 0, 8, 16, 24, 32, 40. 
$15 \times 15$ respectively. For all the plots, the top-left plot is the ROI that is taken from the corner region $(0 \mathrm{~A})$ in Fig. 5.12, the top-right plot is the ROI from edge region (1A), the bottom-right from bulk-region (2A) and bottom left from the central region (3A). Fig. 5.16 are the plots from regions (0B), (1B) and (2B) of the FOV using a $10 \times 10$ pixel array. It is evident from the plots that a $5 \times 5$ pixel array is too small an ROI although it indicates that a $10 \times 10$ array maybe sufficient. There is too much overlap between ROI regions if $15 \times 15$ pixels are used.

The plots only show the values for planes $(0,8,16,24,32,40)$ which are colored red, yellow, green, cyan, blue and purple respectively. The graphs indicate that the signal-to-noise ratio does not improve for the top most plane. This is not surprising because the only information we have about that particular plane are from the in-plane events alone. The central plane, plane 24, improves as more $\theta$ projections are added until all the new information is exhausted. Projection data above a certain $\theta$ is mostly fabricated data. The $\theta$ for which this happens depends on the location within the FOV. Note that planes 8 and 40 are adjacent in the sense that while plane 8 is the 9 th plane from the top, plane 40 is the 8 th plane from the bottom. Thus I expect the results are similar. This is the case for all the plots.

Considering all the plots available, it is hard to justify including more projections after the 10 th one. This corresponds to a projection around $\theta=\frac{\pi}{2}+.5$. From here on, I will use this angle as the limit of the polar acceptance. In order to verify this finding, I simulate a hot extended source over a cooler background. I simulate an elliptical shell phantom with outer semi-major and semi-minor axes $(3.6,1.8) \mathrm{cm}$ respectively, and inner semi-major and semi-minor axes $(1.8,0.9) \mathrm{cm}$ respectively. The phantom is independent of $z$. The phantom is illustrated in Fig. 5.17.

A similar analysis is performed on the extended source phantom as was done on the flood source phantom. The locations where the $\mathrm{S} / \mathrm{N}$ ratio are calculated are indicated by locations $(0),(1)$ and $(2 A)$ and $(2 B)$. The results of the analysis are shown in Fig. 5.18. The data are taken from a variable pixel ROI centered at the locations cited above clockwise starting from the top right. The results again 


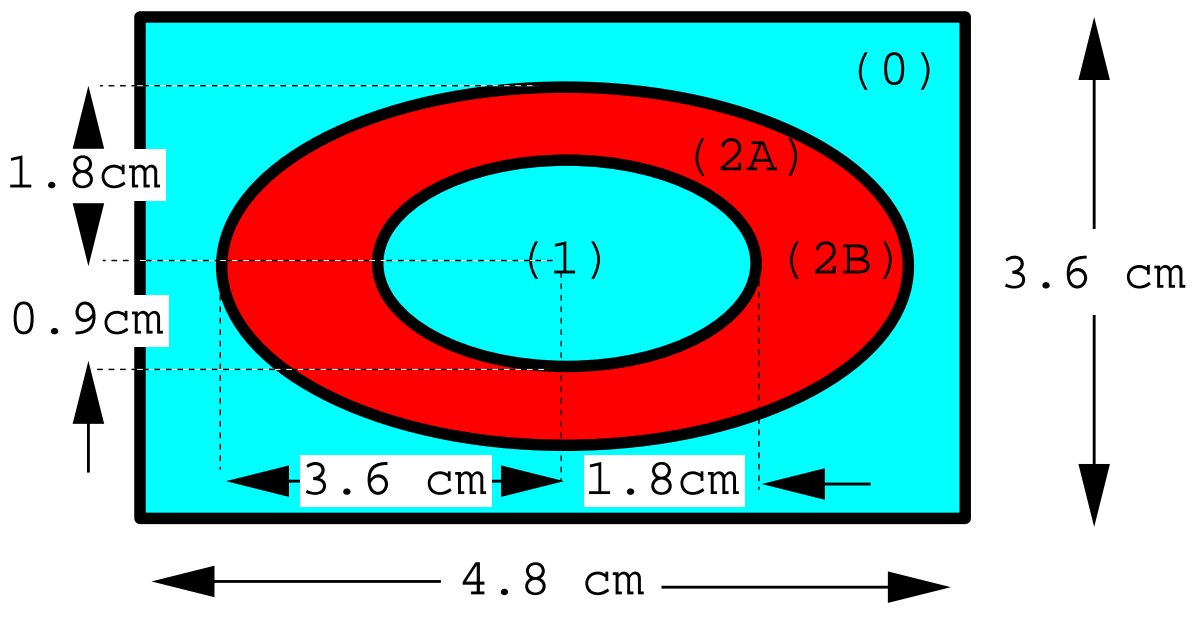

Figure 5.17: Extended Phantom used to verify Polar Acceptance.

indicate that there is little reason to add projections beyond $\frac{\pi}{2}+.5$ radians.

\subsection{Polar Sampling}

Now that I have determined how far in the polar angle we will accept events, I determine at what rate $\theta$ should be sampled. Intuitively, the higher the rate of sampling in any variable, the more faithful the reconstruction will be. Yet again, I need to weigh the benefit versus the cost of sampling at higher rates. The cost of sampling at higher rates is the increase in the size of the data set and increase in reconstruction time.

I investigate two sampling schemes in $\theta$. The fine sampling scheme samples $\theta$ at $\delta \theta=.025$, similar to the minimum angular width in $\phi, \delta_{\phi}^{\min }=0.02427$. The other is the coarse sampling where the angular increments are twice $\delta_{\phi}^{\min }$ as in table 5.2. Note that the polar widths at $\frac{\pi}{2}$ remain $\delta \theta_{\frac{\pi}{2}}=.075$ to minimize zero-efficiency projections bins for the in-plane data. To obtain the fine sampling data, I histogram the events in the very same way as I did for the previous analysis. To obtain the 

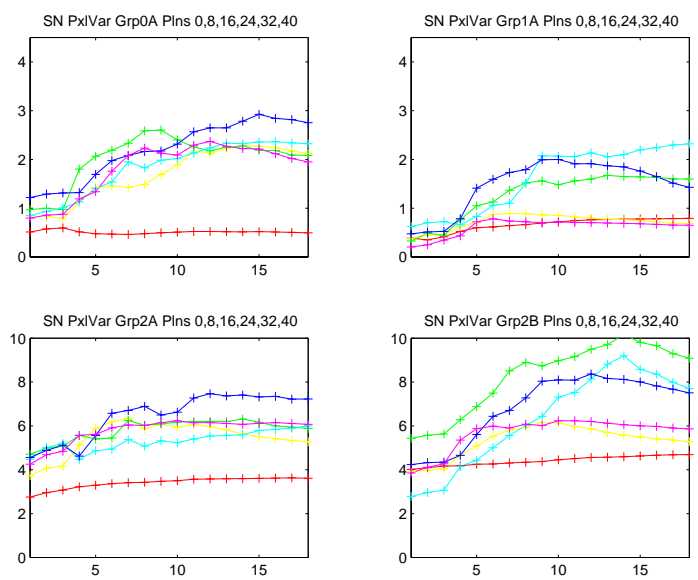

Figure 5.18: Signal to noise ratio of the Flood and Phan001 phantoms. A pixel array of variable size is used, centered at $0 \mathrm{~A}, 1 \mathrm{~A}, 2 \mathrm{~A}$ and $2 \mathrm{~B}$, clockwise from top left. Shown are planes $0,8,16,24,32,40$.

coarse sampling data, however, I merge adjacent angles.

We can use the same figure of merit in this analysis as we did in determining the polar acceptance, namely the $\mathrm{S} / \mathrm{N}$ ratio. However, in the previous analysis, the number of events was not a major concern because we normalize the noise to the signal, in this analysis, there will be systematically more events per projection bin in the coarse sampling set than in the fine sampling set. In fact, we should expect twice as many events in the coarse sampling set as in the fine sampling set. In order to see if the total number of events should be a concern, I simulate data sets with $1 \times 10^{8}$ and $5 \times 10^{7}$ events. Each of these data sets will be histogrammed into two projection sets, one using coarse polar sampling, and another using fine polar sampling. The normalization file is the same data set used in the previous analysis.

At this point, I use $\frac{d}{4}$ sampling, i.e. the projection bins and the voxels have linear dimension of $0.075 \mathrm{~cm}$. The motivation behind this choice is that I am using the fine sampling both in $\theta$ and $\phi$ so it seems natural to use fine sampling in the 
linear dimensions as well. Using fine $\theta$ sampling and $\frac{d}{4}$ sampling effectively increases the projection set size by a factor of eight (8). The reconstruction time increases by nearly an order of magnitude.

There is a complication that comes with $\frac{d}{4}$ sampling that I have not addressed as of yet. Using $\frac{d}{2}$ sampling, the sampling in $\phi$ with its big angular width at $\phi=0$ and $\frac{\pi}{2}$, takes care of the zero-efficiency bins at these projection directions. With $\frac{d}{4}$, however, this scheme is insufficient. Recall that in the case of $2 \mathrm{D}$ reconstruction, going to $\frac{d}{4}$ sampling left holes in the 0 and $\frac{\pi}{2}$ projections. These zero-efficiency bins are a result of sinogram-bin edges being exactly on sampling points in the projection space. The way I handled this is described initially in $§ 4.2 .2$. To fill these empty bins, half of the events in the adjacent high count bin is assigned to the empty bin. The forced continuity of the DOI takes care of the other problem regions in projection space.

In the present case, however, I choose to handle this differently. Instead of allowing the DOI to take on continuous values only along the long axis of the crystal, I allow it to populate the entire volume of the DOI bin. This means that the interaction point takes on a random location within the $[0.3 \times 0.3 \times 0.375] \mathrm{cm}^{3}$ volume of the DOI bin. We did not use this option in the $2 \mathrm{D}$ reconstruction primarily because this procedure is effectively convolving the detector response with a nonlocal kernel which is difficult to deconvolve from the reconstructed image. I do it presently because it is simple. As I will show in the following section, the most significant effect of this procedure is a broadening of the PSF in the axial direction, i.e. the $z$-axis. At face value, this would seem to suggest that I could have used the same algorithm in the $2 \mathrm{D}$ reconstruction.

The results of the analysis are shown in Fig. 5.19. I calculate the mean pixel value and the corresponding standard deviation for the entire plane (there are now 96 planes). Note that the means of extreme planes are consistently underestimated while their variance are over estimated when compared to the mid-planes. The overestimation of the variance is understandable and is a result of the lack of events 


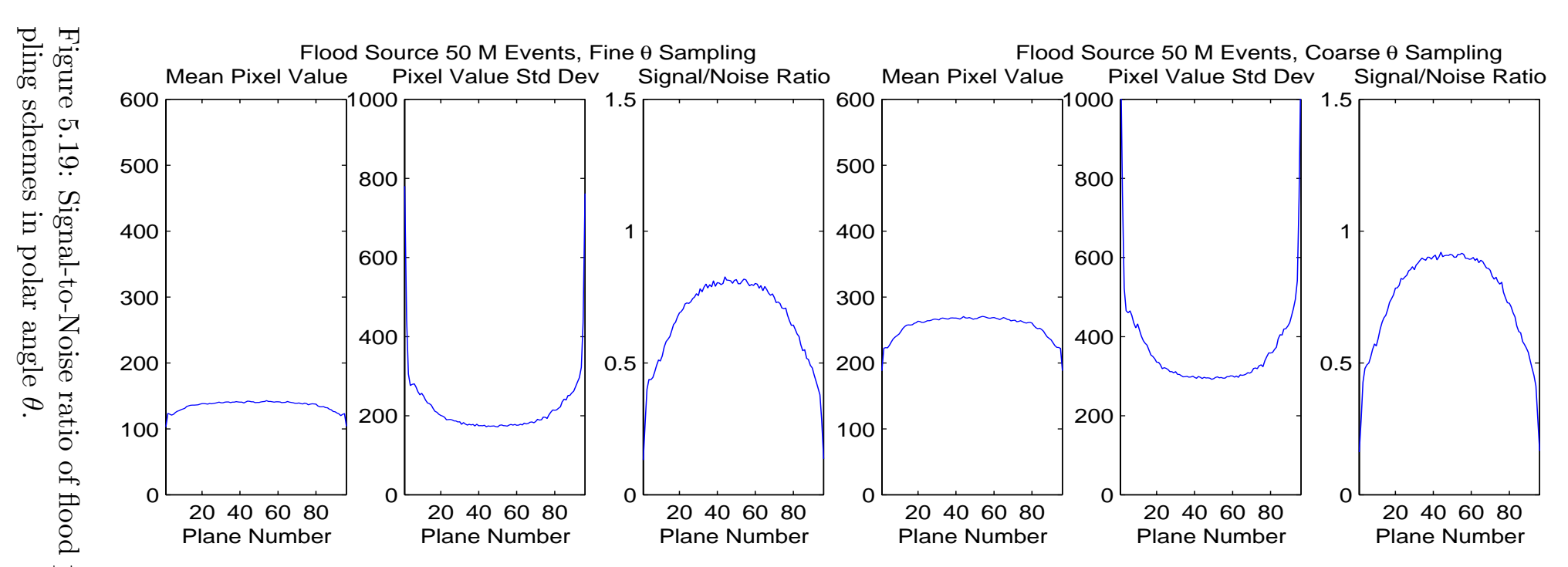

Flood Source $100 \mathrm{M}$ Events, Fine $\theta$ Sampling

Flood Source $100 \mathrm{M}$ Events, Coarse $\theta$ Sampling Mean Pixel Value Pixel Value Std Dev Signal/Noise Ratio

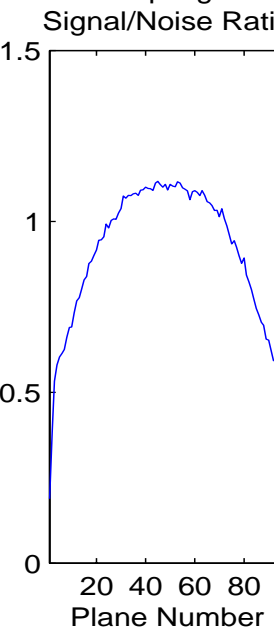

Mean Pixel Value Pixel Value Std Dev

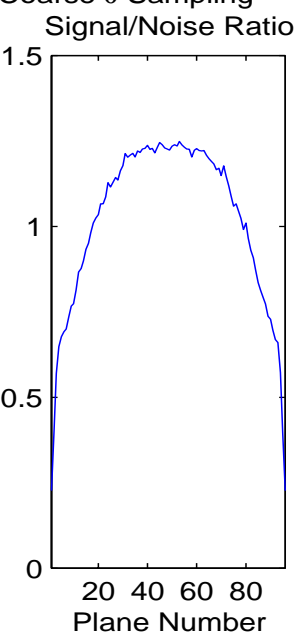


in those planes compared to the other planes. The underestimation of the mean, however, is puzzling. I will address this issue in the next section. For the time being, since we compare the same planes of different images, the results that we obtain from these analysis should be valid.

Comparing the $\mathrm{S} / \mathrm{N}$ ratio plots in Fig. 5.19, between it appears that for a constant number of events, the $\mathrm{S} / \mathrm{N}$ ratio analysis does not distinguish between the two polar sampling schemes. The increase in the signal between planes reconstructed using coarse sampling versus fine sampling is consistent with the number of events increasing by a factor of 2 . The noise appears to increase as the signal does, resulting in very little change in the $\mathrm{S} / \mathrm{N}$ ratio.

\subsubsection{Resolution Test}

Because the previous analysis failed to distinguish between the two polar sampling schemes, I perform another analysis to attempt to determine whether the benefit of fine polar sampling outweighs the doubling of the data set. For the second analysis, I use the resolution of the PSF as the figure of merit. I simulate equal intensity point sources at various locations in the FOV. The $x y$ locations are the same as those simulated in $\S 4.2 .3$. The $z$ values of the point sources are $z=(-$ $3.45,-2.85,-2.25,-1.65,-1.05,-0.45,0.15,0.75,1.35,1.95,2.55,3.15)$, i.e. they are located in plane 2 and every 8th plane after that until plane 90. The point sources are "bathed" in a low background flood source. A total of $50 \mathrm{M}$ annihilations are evenly distributed among the 84 point sources, giving each source approximately 6 $\mathrm{M}$ annihilations. Another $50 \mathrm{M}$ annihilations are distributed evenly in the warm bath. Fig. 5.20 shows the middle plane of the reconstructed image.

In order to isolate the point sources, I use a threshold on the image. I calculate the mean and the standard deviation of the voxel values in the entire image and perform the analysis that I will describe for voxels that are $3,4,5,6$, and $7 \sigma$ from the mean. Once the threshold has been applied to the image, I begin to search 


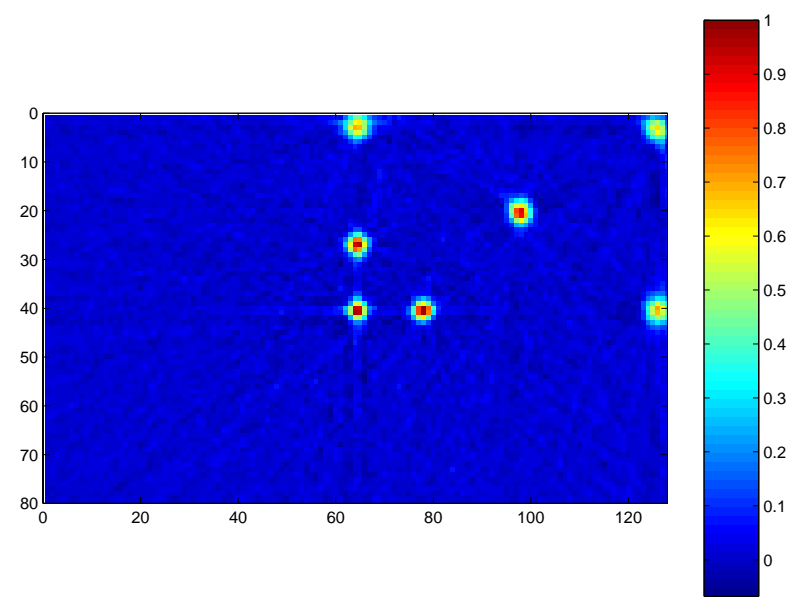

Figure 5.20: Middle plane of Point Sources Phantom.

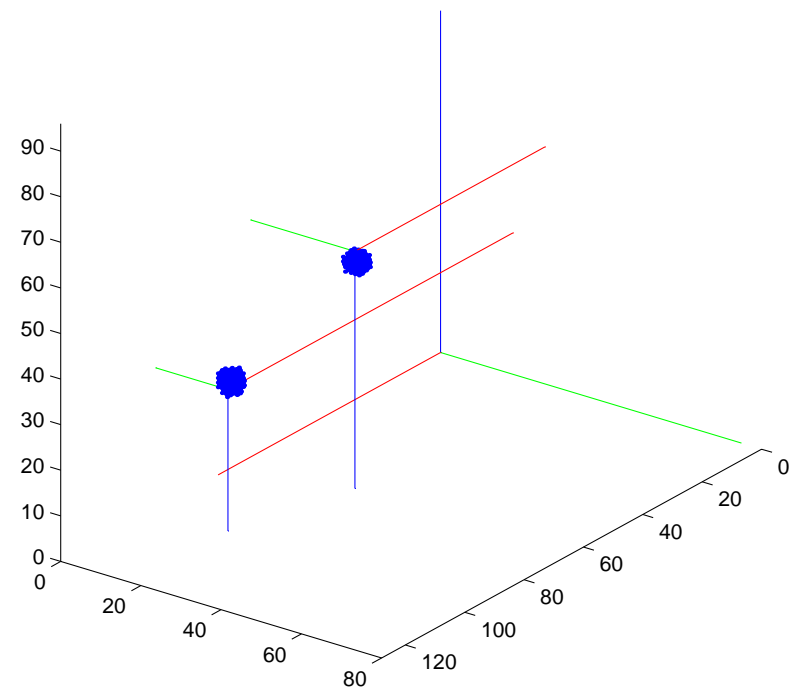

Figure 5.21: Point Sources carved out by algorithm as described in text. The axes indicate the voxel indices. 
for voxels with non-zero values, top plane first, starting from the top left corner of the plane. When I find a non-zero voxel, I search for all the contiguous non-zero voxels. If there are more than four voxels in this set, I define this as a reconstructed point source. I do this until I've carved out all the point sources from the image. At this point, I have the voxels that comprise each reconstructed point source. Fig. 5.21 shows typical "blobs" carved out by this process. With each of these point sources, I sum the voxel values in two indexes, leaving the integrated voxel intensity along one axis corresponding to the third index. This voxel intensity profile in this remaining axis is super-sampled by 4, linearly interpolating between the non-zero sampling points. I then find the maximum and the two points on either side of the maximum at which the value of the profile drops to below half of this maximum. I define the FWHM as the distance between these two points.

Fig. 5.22 shows the result of the analysis. I show the results for two values of the threshold. The top left plot shows the results when the threshold equals $5 \sigma$. The $x$-axis is the point source index. The point sources are numbered such that groups of seven belong in the same plane, i.e. the first seven indexes correspond to the point sources in plane 2, the second seven are in plane 10, etc. Notice the regularity in the resolution pattern as the index number increases. The lowest points in the groups of seven correspond to point sources at the center of the tomograph. There are four curves in the plot. The results of the current analysis are in red and yellow, corresponding to the fine and coarse polar-sampling reconstruction respectively. The magenta curve in the plot immediately below is the absolute difference between these two curves. The conclusion is that on average, with respect to $R_{x}$, the width of the PSF in $x$, the difference between fine and coarse sampling approximately less than $.375 \mathrm{~mm}=\frac{d}{8}$. This is equivalent to a null result, i.e. this analysis does not distinguish between fine and coarse polar sampling. In fact, the same can be said for $R_{y}$ and perhaps even $R_{z}$, although in the case of $R_{z}$, the fine sampling reconstruction has a systematically lower value.

This null result prompted me to see if this was a consequence of the fact 
that I allow the interaction point to be a random location within the entire DOI volume. Recall that when I reconstructed images in $\mathbf{R}^{2}$, the interaction point was a random location along the main axis of the crystal within the DOI volume. The second pair of curves, the green and the blue, in the top left plot are the PSF of the reconstructed point sources when the interaction point is only allowed to be along the main axis of the crystal. The cyan curve in the plot immediately below is the absolute difference between these two new curves. The conclusion is the same. We are sacrificing neither resolution nor signal-to-noise ratio by coarsely sampling the polar angle. Increasing the threshold to $7 \sigma$ does not change the conclusion.

\subsubsection{Normalization Revisited}

The overestimation of the noise in the top and bottom planes is understandable. The underestimation of the mean voxel value, however, is puzzling. The top left plot in Fig. 5.23 is the mean voxel value of the reconstruction of the ideal noiseless data, scaled such that the mean voxel value of the 48 th plane is 1 . The $x$ axis of the plot corresponds to the plane index. Notice that the plot is essentially flat across the different planes. The slight decrease in value at the extreme planes can be attributed to the overshoot of the FFT. Recall that we are reconstructing an object that has finite support with sharp edges, i.e. the phantom has a discontinuity from finite value to 0 as you cross the edges of the FOV.

One possible explanation for the underestimation of the mean value at the outer planes is that I did not take into consideration the statistics of the individual voxels in the plane, i.e. I calculated the unweighted mean of the voxel values. If the statistics of each individual voxel are indeed different enough from one another to cause this underestimation, then I can try to see if I can modify the ideal noiseless data to reconstruct into an image that underestimates the outer planes as well. To see if this is indeed the case, I add Poisson noise to the ideal data and reconstruct. I reconstruct three images, one in which the ideal data has been scaled such that it 

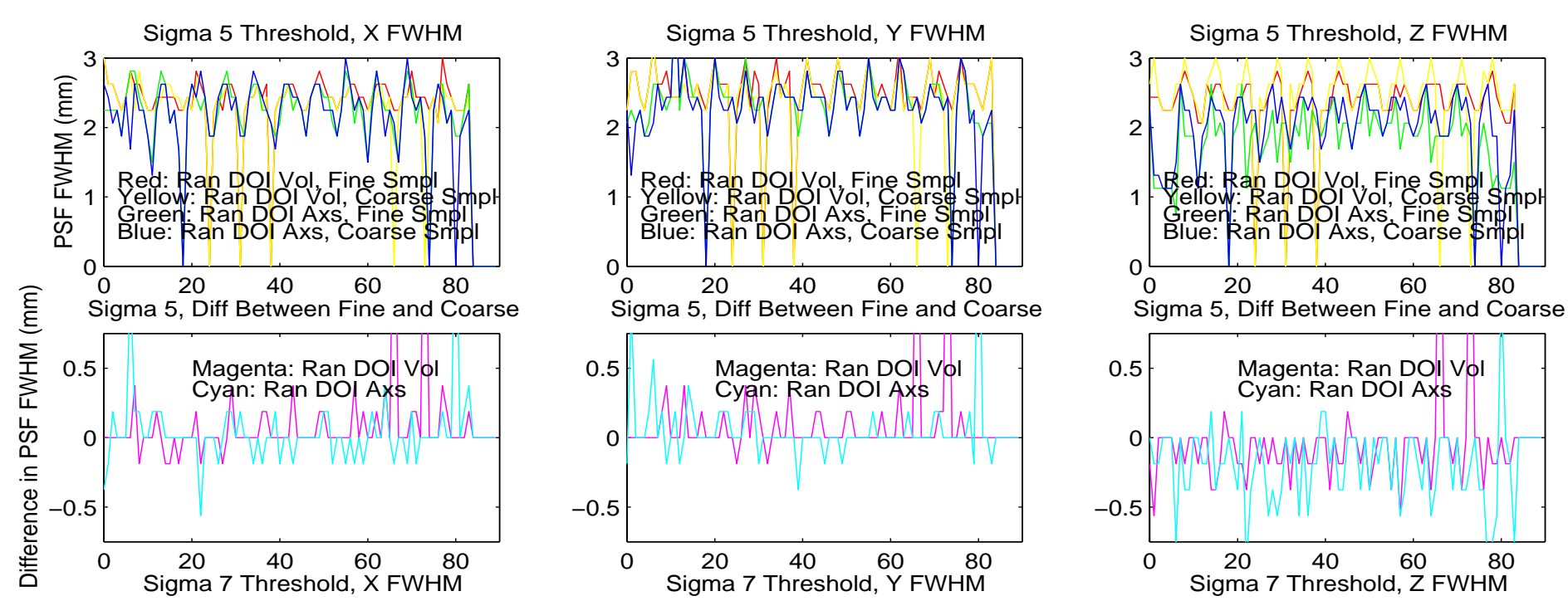

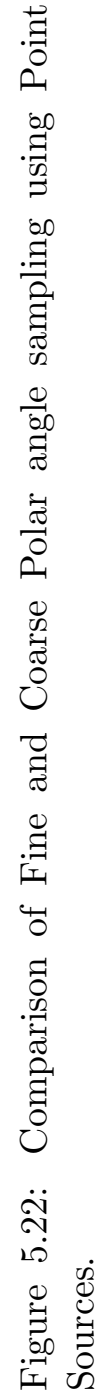
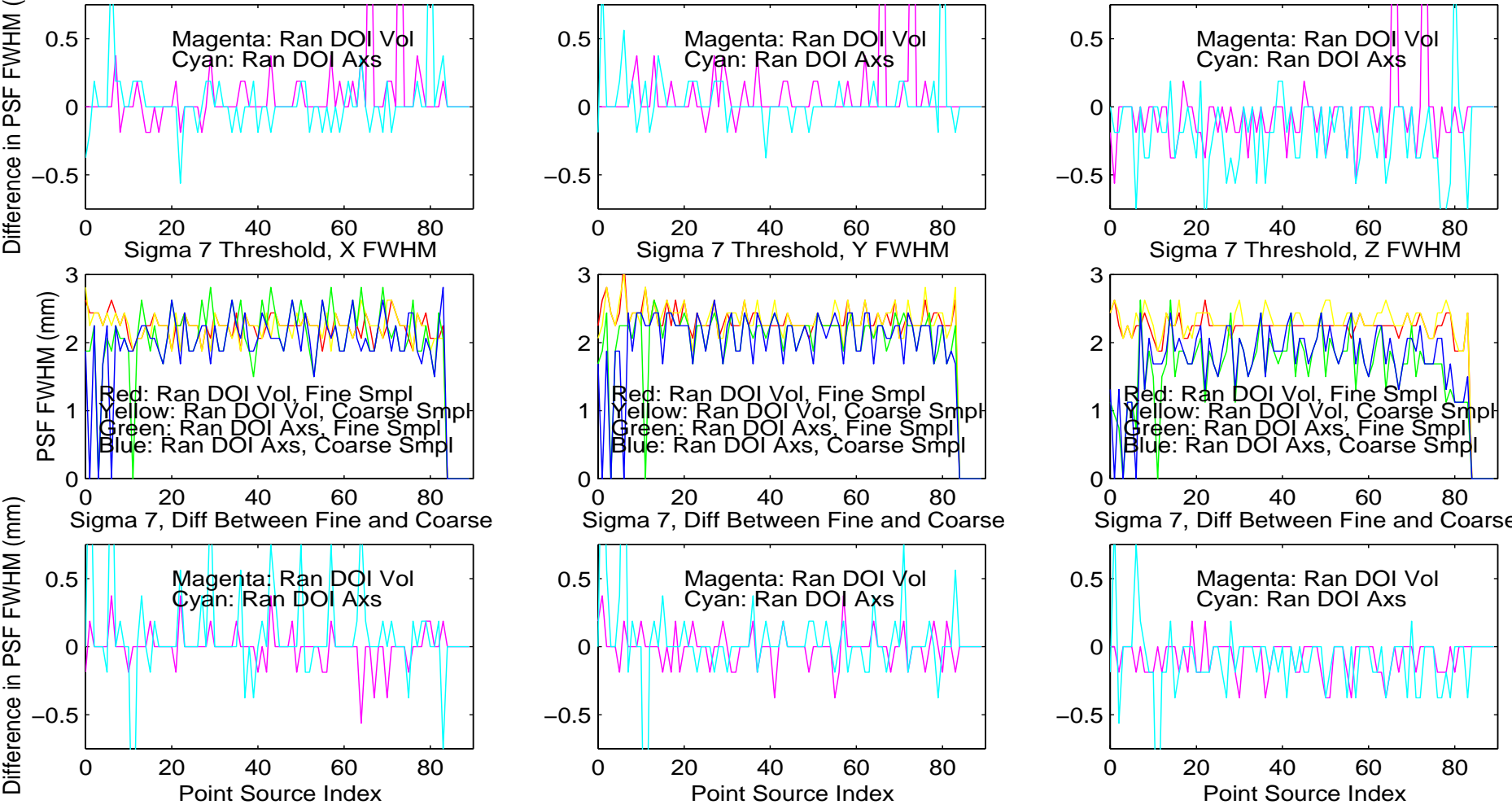

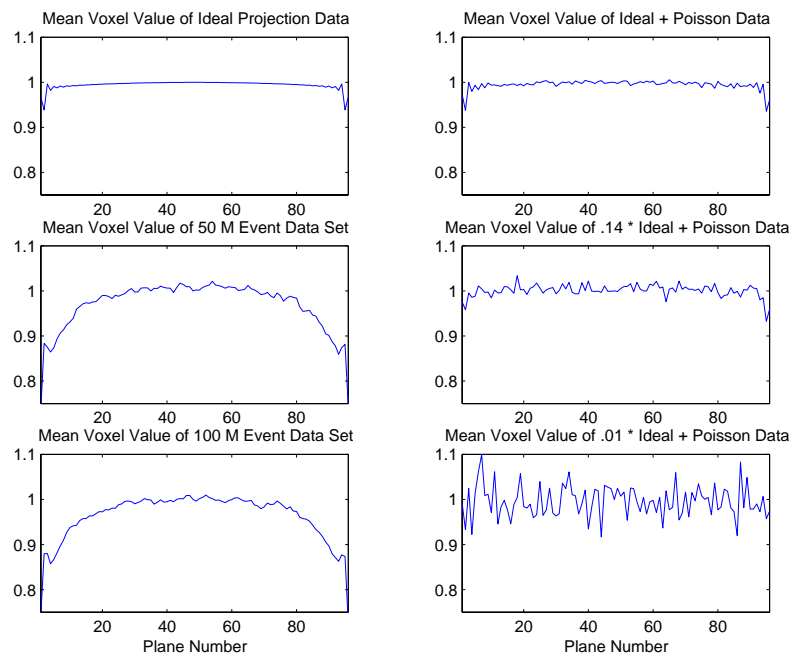

Figure 5.23: Comparison of ideal means.

is equivalent to $10^{9}$ events, one with $10^{8}$, and another with $10^{7}$.

The right-hand plots in Fig. 5.23 show the mean voxel values of the images with $10^{9}$ events (top), $10^{8}$ events (middle) and $10^{7}$ events (bottom). These data sets corresponds to events with the less, equal, and more noise when compared to the 100M-event data set respectively. Note that while the increase in the noise characteristics of the image is readily apparent (when compared to that of noiseless data), I cannot see any underestimation in the mean voxel values of the outer planes.

Another possibility is that the normalization factor that I use is incorrect. Recall that the normalization factor is determined by the bin-by-bin ratio of the line integral of the FOV to a high-count flood data set, i.e. $n_{i}=\frac{a_{i}}{f_{i}}$, where $a_{i}$ is the line integral of a solid box with the dimensions of the FOV and $f_{i}$ is the number of events in bin $i$ detected from a high activity flood source. This corresponds to multiplying the projection data by $\frac{1}{E\left[f_{i}\right]}$, where $E[*]$ is the expectation operator, when in fact, I should multiply by $E\left[\frac{1}{f_{i}}\right]$. Fig. 5.24 shows the ratio of $E\left[\frac{1}{\lambda_{i}}\right]$ and $\frac{1}{E\left[\lambda_{i}\right]}$ as a function of the expected number of events. $\lambda$ has Poisson statistics. Note that the ratio tends 


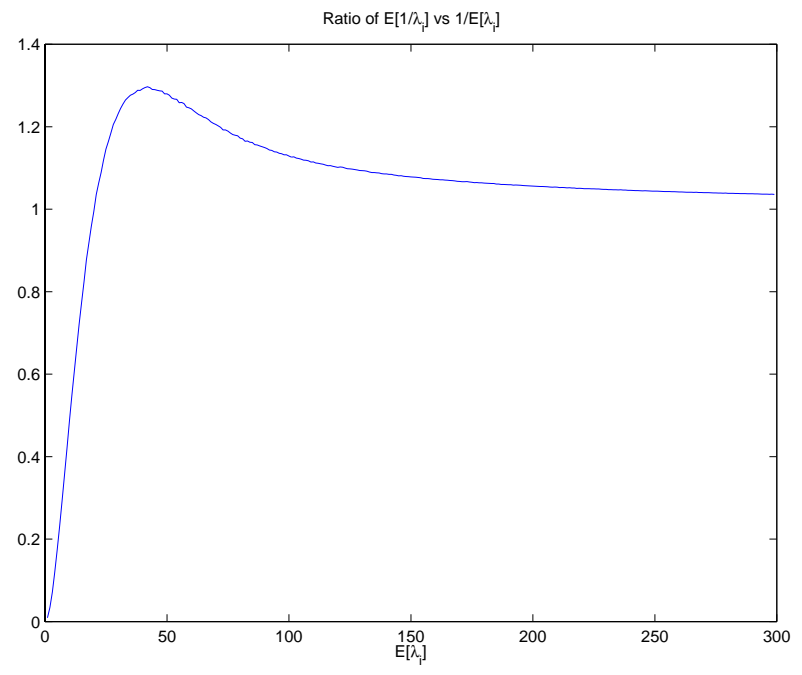

Figure 5.24: Ratio of $E\left[\frac{1}{\lambda_{i}}\right]$ and $\frac{1}{E\left[\lambda_{i}\right]}$ if $\lambda_{i}$ is Poisson distributed.

to 1 for increasing values of $E\left[\lambda_{i}\right]$.

Application of the correction factor improves the uniformity of the mean voxel value of the planes. Fig. 5.25 shows the improvement in the mean voxel value of each plane if the normalization is corrected for the bias. However, the improvement is not complete. This reconstruction, as in the previous reconstructions, uses a highcount flood data set corresponding to $8 \times 10^{8}$ annihilations. This suggests that I need to increase the number of events in the high-count flood source. Fig. 5.26 shows the improvement in the uniformity of the mean voxel value as more events are used for the calculation of the normalization factors. It appears that $3.5 \times 10^{9}$ annihilations are a sufficient number of events for the normalization file.

The lack of events in the normalization file does not change the results of the previous sampling study. In the first place, I compare corresponding planes between reconstructions that used data normalized with the same normalization factors. The signal-to-noise ratio changes by a few percent but the overall conclusion remains that the gains brought about by finely sampling the polar angle does not justify the 


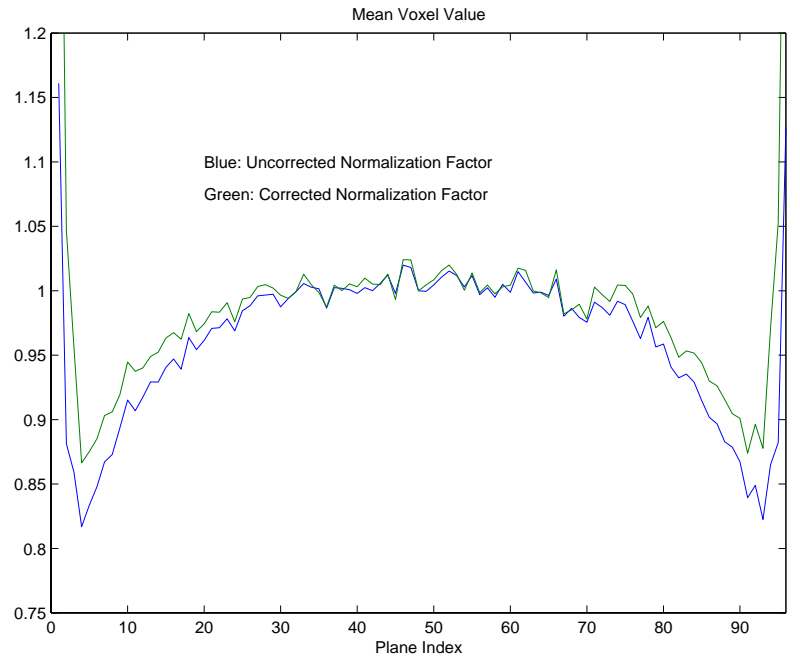

Figure 5.25: Mean voxel value as a function of plane index. Note the improvement in the uniformity if the normalization values are corrected for the difference in $E\left[\frac{1}{\lambda_{i}}\right]$ and $\frac{1}{E\left[\lambda_{i}\right]}$.

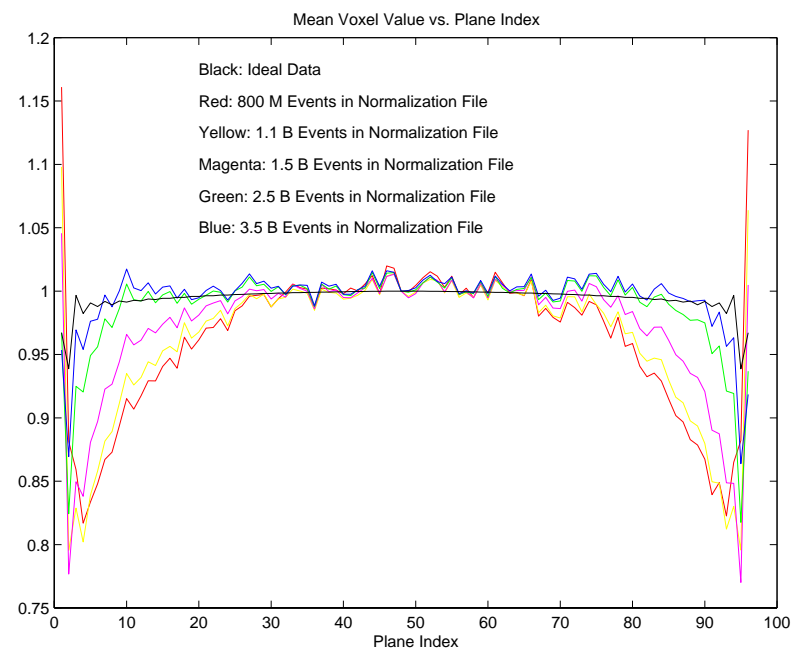

Figure 5.26: Mean voxel value as a function of plane index. Note the improvement in the uniformity as more events are used for the normalization file. 
doubling of the data set and doubling of the reconstruction time.

\subsection{A Sample Reconstruction}

To get a flavor of the PEM reconstruction with the parameters that I have just determined, I reconstruct the data from the elliptical extended source phantom described in Fig. 5.17 using the coarse sampling scheme. I simulate $5 \times 10^{7}$ annihilations both in the colder background and in the hotter ellipse, and the data file is normalized using the normalization file with $3.5 \times 10^{9}$ events. Fig. 5.27 shows the even planes of the top half of the reconstructed FOV. The images are scaled to show the relative intensities of the regions. The results from $\S 5.5 .1$ indicates that the resolution is expected to be isotropic. The present reconstructed images indicate that the noise is not. Note that the ellipse is difficult to discern in Plane 0, and while I can identify the ellipse in Plane 2, the noise blurs its boundary.

To quantify the accuracy of the reconstruction, I calculate the activity ratio between the hot phantom and the warm background. The ratio of the areas of the source and the background is $15.3: 57.6=0.265$. Thus, the expected ratio of the mean voxel value between the source and background is $\frac{1-0.265}{57.6-15.3}: \frac{1+0.265}{15.3}=1: 4.76$. Fig. 5.28 shows the mean voxel values in the phantom and in the background for the different planes in the FOV. While the mean voxel value within the phantom is uniform, the colder background suffers from the familiar underestimation at the extreme planes. The result is that the ratio is artificially higher in those planes.

\subsection{Realistic Camera Geometry}

Now that I have extended the irregular sampling reconstruction to the ideal camera geometry, I begin to include real-world effects such as the acollinearity of the photons and Compton Scattering in the detector modules. I also include photon sharing in the detector modules and calculate the DOI by taking the ratio of the 


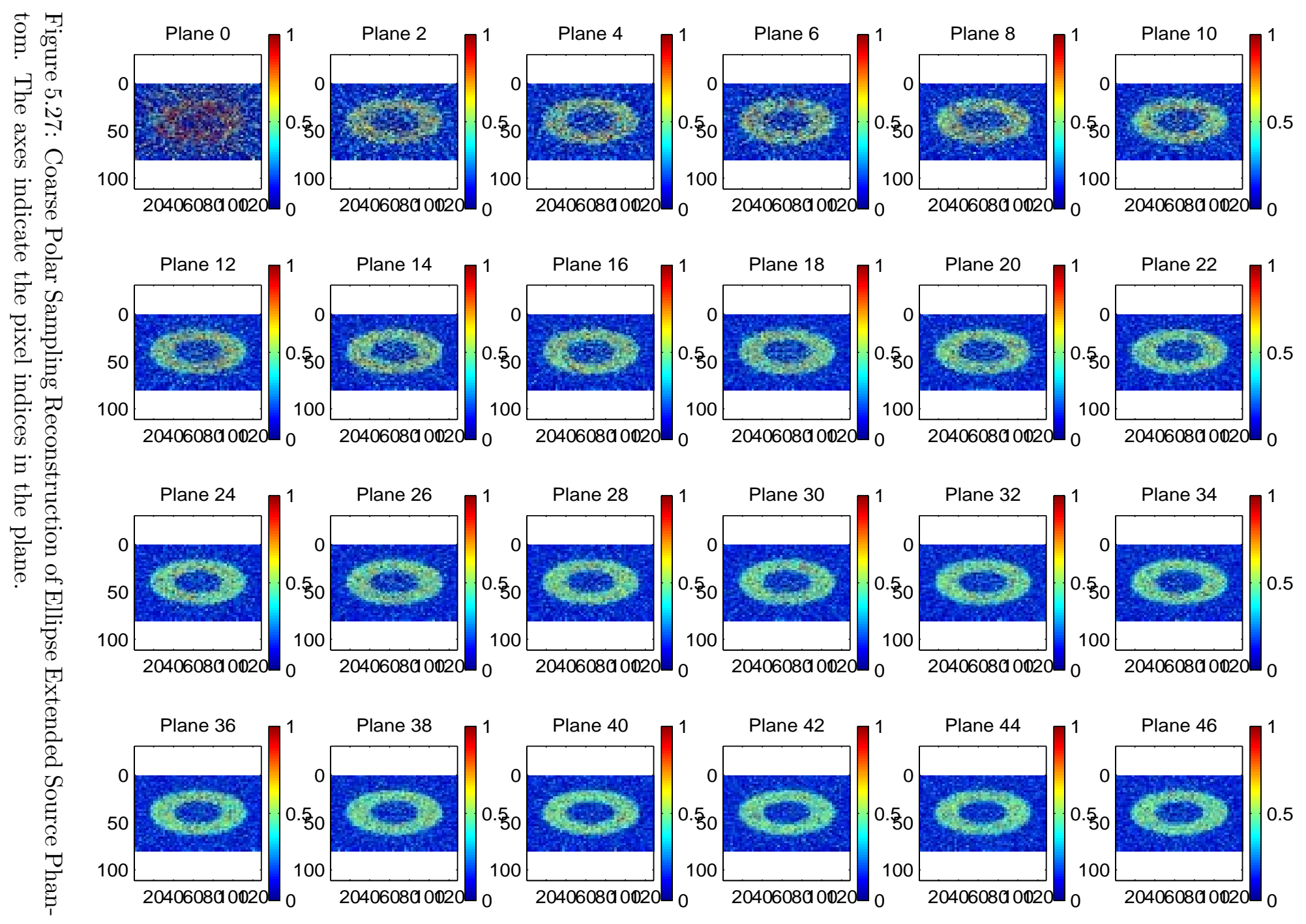



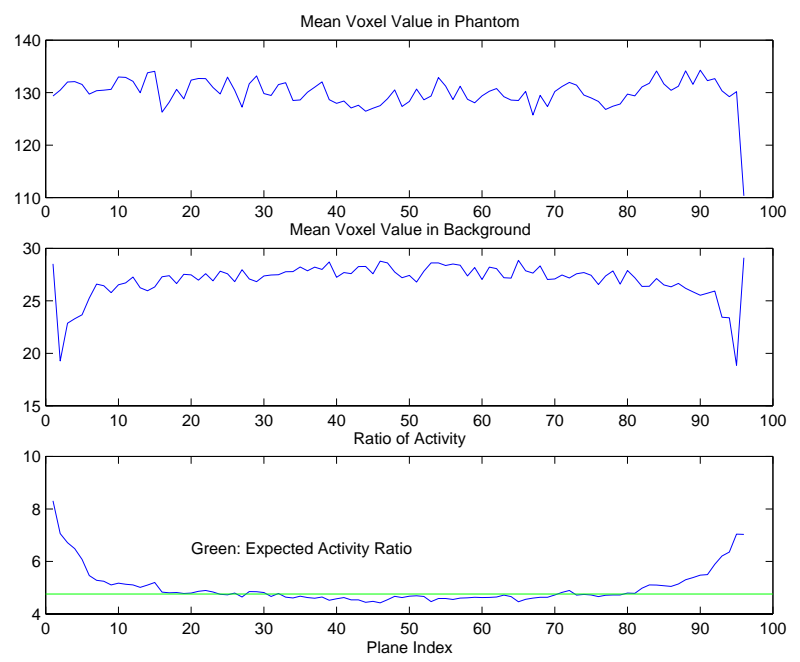

Figure 5.28: Plane statistics of reconstructed extended ellipse phantom.

signal in the PD to the sum of the signals in both the PD and PMT. Finally, I include gaps between detector modules in anticipation of the packing limitations of the camera.

Inclusion of gaps between the detector results in a loss of approximately $\frac{1}{3}$ of the events. The gaps reduce the active surface of a detector module to $\left(\frac{8}{9}\right)^{2}=79 \%$. Two modules in coincidence is reduced by approximately $62 \%$. If the photofraction of LSO at $511 \mathrm{keV}$ were $100 \%$, with the attenuation constant were $1.2 \mathrm{~cm}$, the detection efficiency of the PEM detector is $28 \%$. (Recall that the efficiency of the PEM camera if the modules had black crystals is $39 \%$ (Table 5.1)). I used these conditions to simulate the Ideal Detector. Thus, with the drop in geometric acceptance, I anticipate a detection efficiency of $19 \%$.

Inclusion of Compton Scatter in the detector modules means that I will need to use energy cuts in determining which coincidence events to keep. Fig. 5.30 shows the energy spectrum of the detected events for four operating conditions for the camera. Light statistics are modeled as Gaussian with a standard deviation 


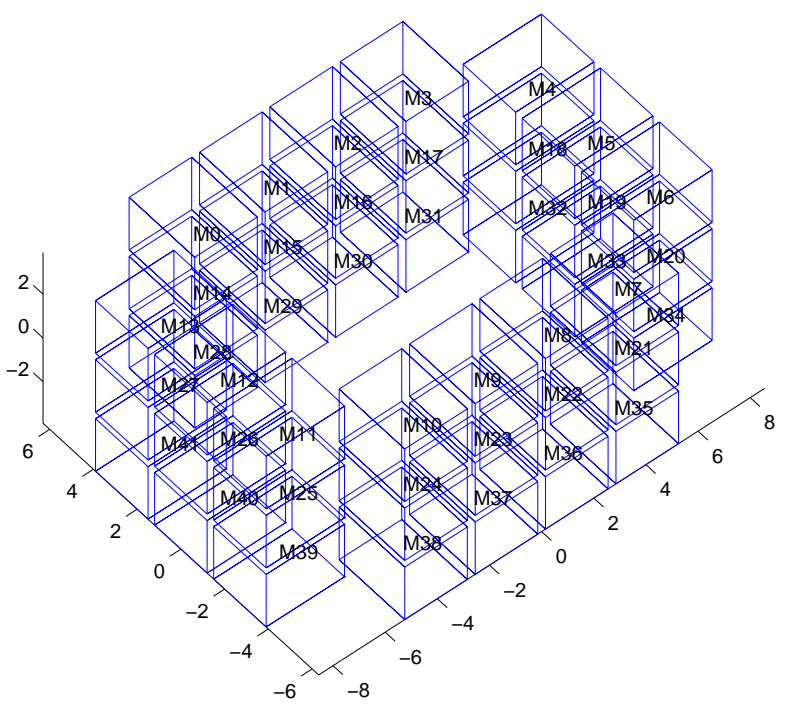

Figure 5.29: Wireframe image of the realistic PEM Detector Geometry. The longest axis is the $x$ axis while axial direction through the FOV is the $z$ axis. The modules are grouped together in banks corresponding to the four sides of the rectangular FOV. Note the gaps between each module in the camera. The axes are in units of $\mathrm{cm}$. 


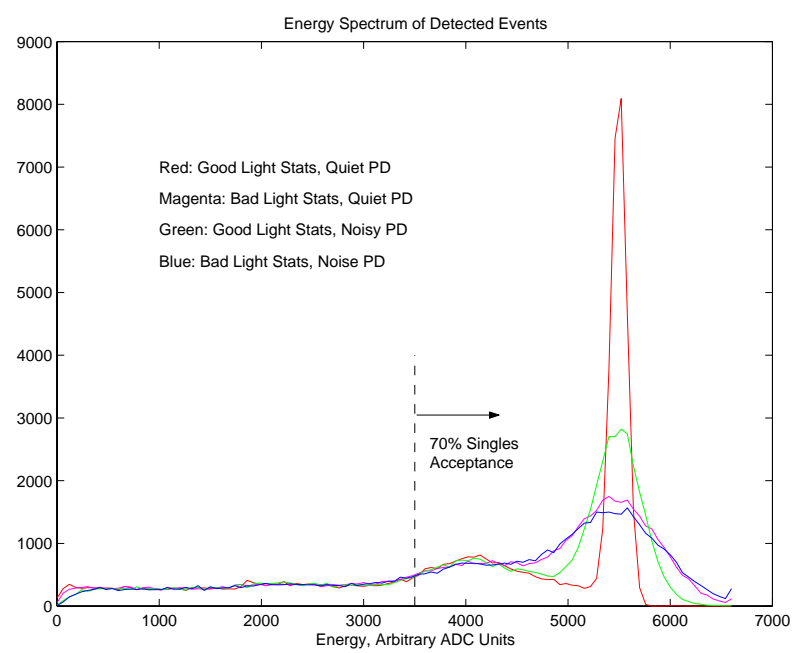

Figure 5.30: Energy spectrum of detected events. Plotted is the sum of the PMT signal and the highest PD signal in the array.

given by the square root of the number of photons generated. To simulate bad light output, I multiply this standard deviation by 30 . To simulate a noisy PD, additional Gaussian noise is added in quadrature. I simulate the worst case shown here, which corresponds to approximately a $21 \%$ FWHM energy resolution. This is consistent with the resolution we expect from the camera [66].

The energy window used in conventional PET scanners is $350 \mathrm{keV}$ to 650 $\mathrm{keV}$. I do not put a high-energy threshold on the energy of the event. However, for the lower energy threshold, I put a cut at 3500 ADC counts, corresponding to 350 $\mathrm{keV}$, to keep $70 \%$ of the singles events and accept $49 \%$ of all detected events. Thus the overall detection efficiency of the simulated detector is $9 \%$.

So far, whenever the DOI is needed to be discretized, I have allowed the DOI to take on a random value within "DOI bins" that are $3.75 \mathrm{~mm}$ long, corresponding to dividing the $30 \mathrm{~mm}$ crystals into 8 DOI bins. With the modeling of a more realistic energy resolution in the PD and the PMT, a DOI resolution that constrains the interaction point to $3.75 \mathrm{~mm}$ is too optimistic. Fig. 5.32 shows the absolute difference 


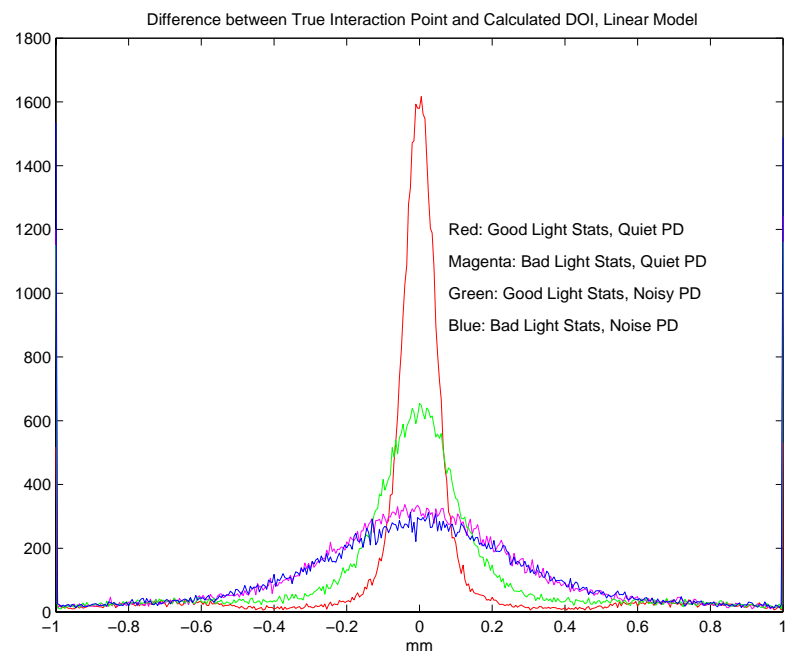

Figure 5.31: Difference between the true depth and the calculated depth for the conditions in Fig. 5.30. The distributions have widths of $3.2 \mathrm{~mm}, 4.7 \mathrm{~mm}, 4.5 \mathrm{~mm}$, $5.3 \mathrm{~mm}$ FWHM respectively.

between the true DOI and that which is calculated using the depth estimator $\Gamma$. Note that as the energy resolution worsens, the DOI resolution worsens as well. In particular, since I will be simulating the detector with an energy resolution of $21 \%$, I will use DOI bins that are $5 \mathrm{~mm}$ in length instead.

The modeling of a more realistic energy resolution also has another consequence in determining the DOI that I briefly alluded to in $\S 4.3$. The depth estimator $\Gamma$ from which the DOI is calculated can take on values outside its bounds had the detectors been perfect. Fig. 5.32(A) shows the distribution of $\Gamma$ for the module with $21 \%$ energy resolution. (B) shows the calculated DOI. Note that $13 \%$ of the events will be assigned a DOI that is outside the crystal. In these cases, the event is assigned to the closest end of the crystal. (C) shows the distribution of the DOI after the redefinition of the depth in cases where $\Gamma$ is outside its ideal boundaries.

Fig. 5.33 shows the projection data for $4.6 \times 10^{9}$ coincidences from a flood source. Note that the gaps between the modules are evident by the large contiguous 

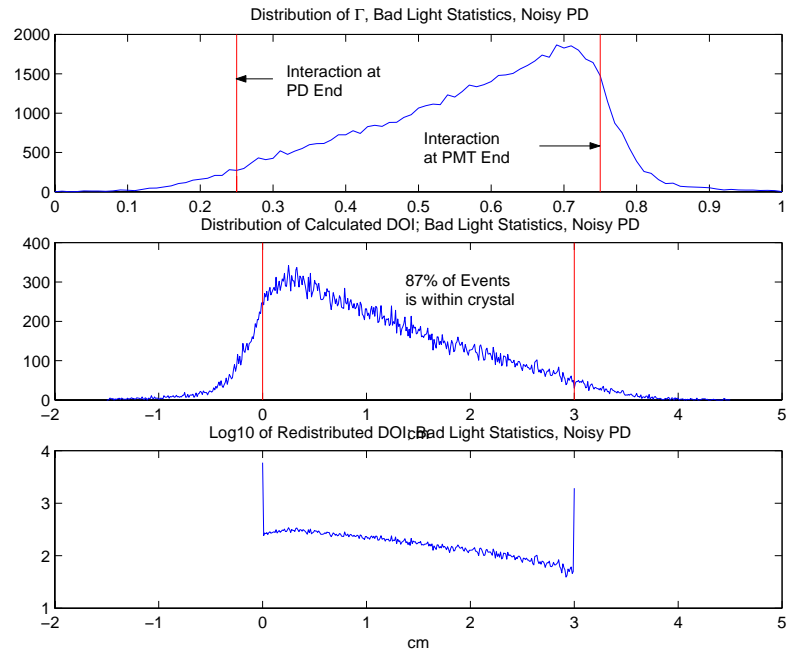

Figure 5.32: Depth estimator $\Gamma$ and redistributed DOI.

unsampled regions of the projection planes. In addition to the missing data due to the gaps between modules, the data also shows the effect of the requirement that the interaction point be along the long axis of the crystal. This is evidenced by the local non-uniformity of the data in the off-plane projections. This problem will be partially eliminated by the normalization. However, there are off-plane projection bins that are not sampled. I treat these bins like the truncated data. I estimate the data in these bins from a scaled forward projection of the initial low-statistics 2D reconstruction of the in-plane data. This was not possible in the $2 \mathrm{D}$ case because there is no redundant information in $2 \mathrm{D}$.

\subsubsection{Reconstructing Initial 2D Estimate}

The reconstruction that I did in Chapter 4 was in anticipation of the problems that the $3 \mathrm{D}$ data contains. The initial $2 \mathrm{D}$ estimate that I will use for the 3DRP reconstruction will come from the projection planes with $\theta=\frac{\pi}{2}$, i.e. the set of projections in the first row of Fig. 5.33. This set of data suffers from the missing gaps 

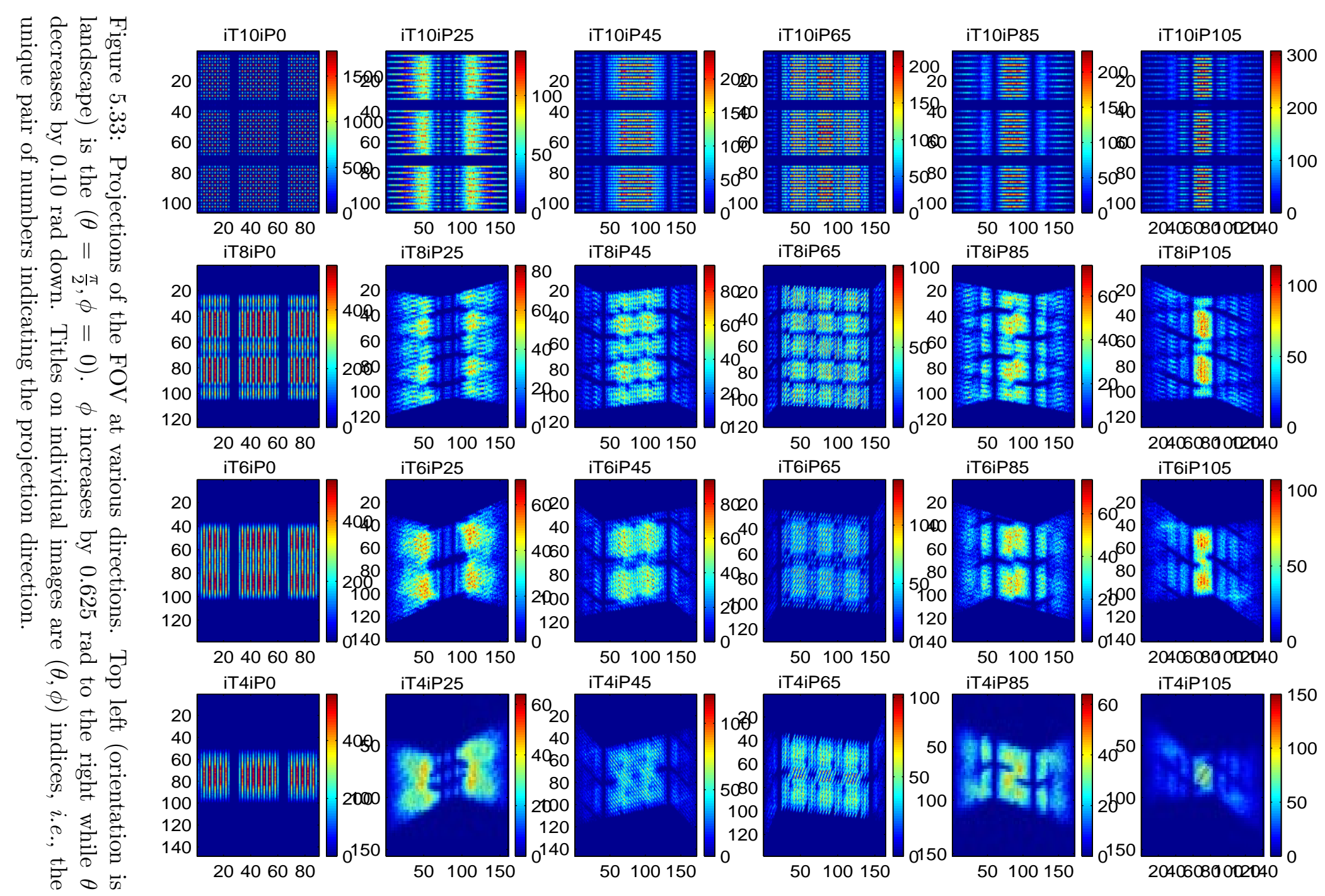

iT8iP105
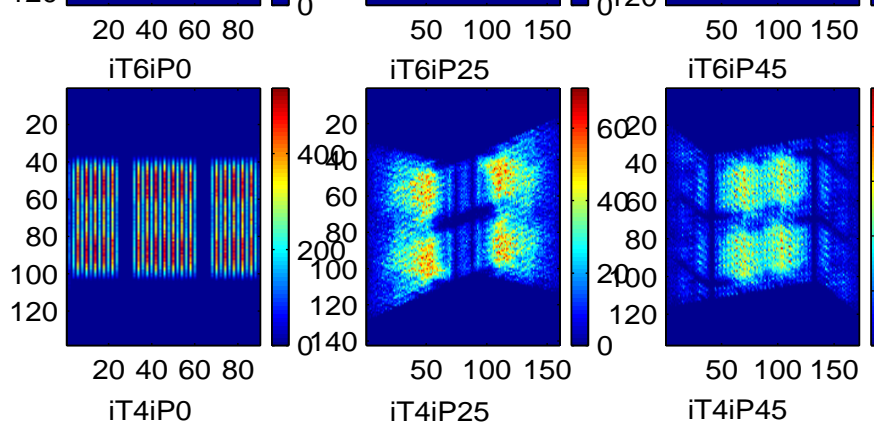

T6iP65

50100150
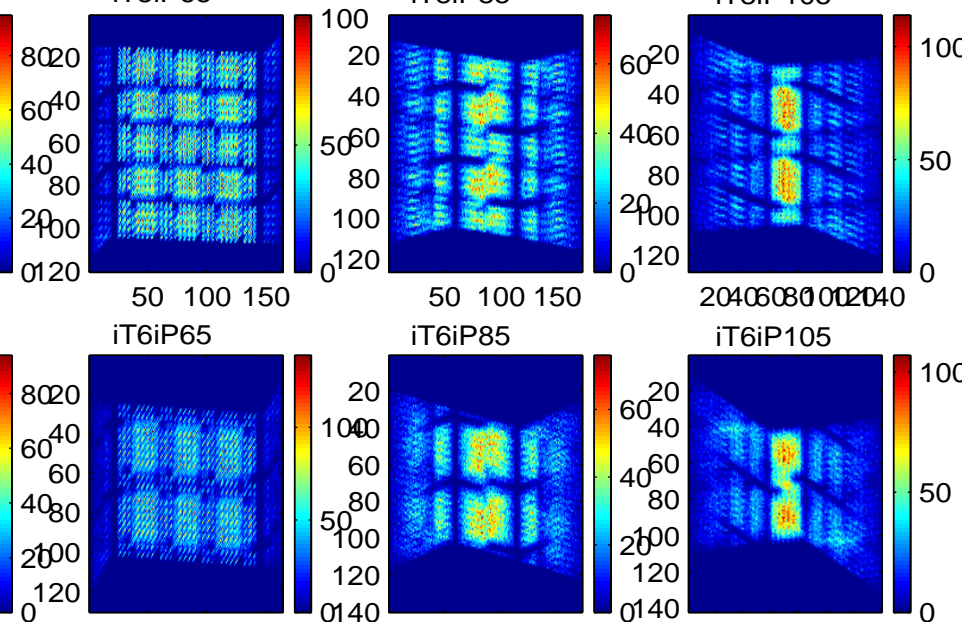

iT6iP85

20406080002040
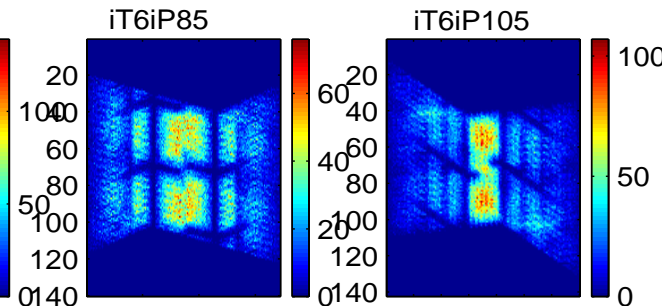

50100150

50100150

50100150

20406080002040
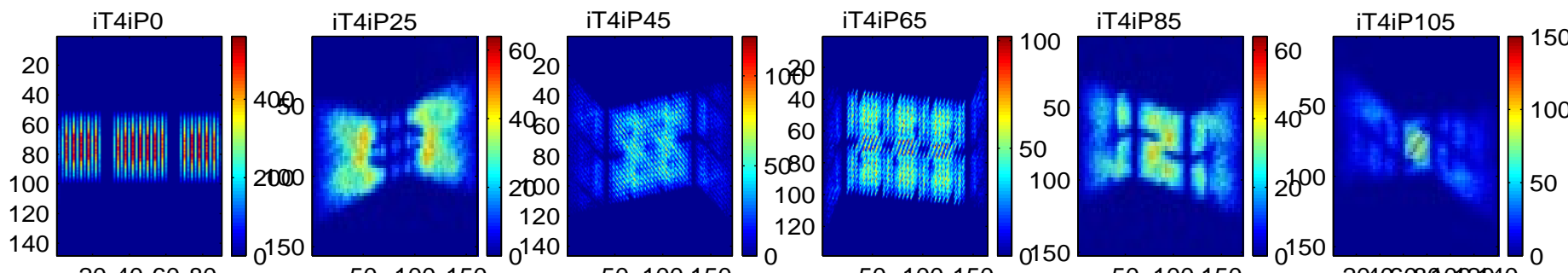

50100150

50100150

20406080002040 

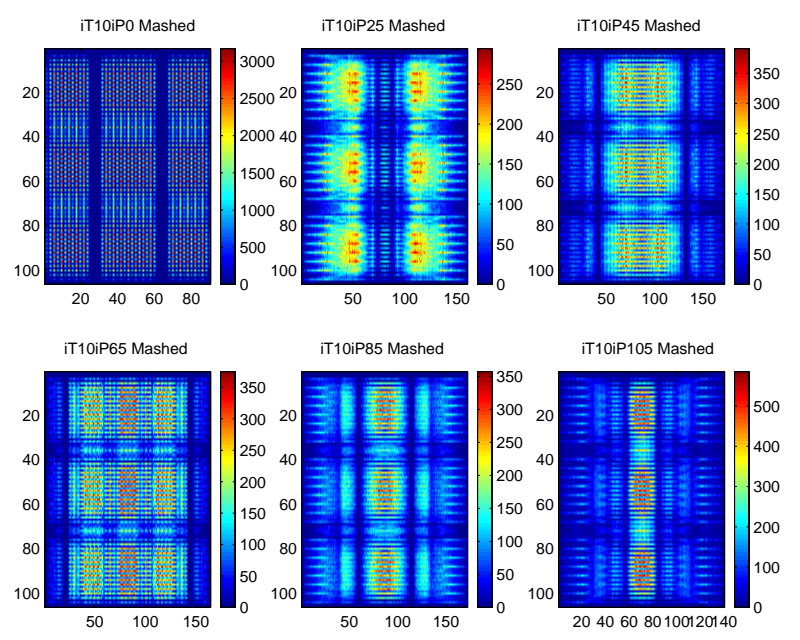

Figure 5.34: Mashed Projections. These projections are the sum of the $\theta=\frac{\pi}{2}$ and the first off-plane projections. They have $\phi$ s equal to $0,0.657440$ and 1.142807 in the top row and $1.780369,2.265736$ and 2.751104 in the bottom row.

and the requirement that the interaction point be along the long axis of the crystal. The gap problem is clearly seen in the projection $i T 10 i P 0$, corresponding to the $10^{t h}$ projection in $\theta$, i.e. $\theta=\frac{\pi}{2}$, and the $0^{\text {th }}$ projection in $\phi$, i.e. $\phi=0$. The $\phi$ values of the other projections are given in Tables 5.3 and 5.4. The requirement that the interaction point be defined along the long axis of the crystal results in the alternating bright and dark bands in the rest of the in-plane projections. This basically means that some of the planes are defined to have a lower efficiency that will need to be corrected.

The reconstruction procedure used in $\S 4.3 .2$ was designed to take care of the vertical gaps in the projection planes. Recall that the $2 \mathrm{D}$ data is essentially the column sum of these projection planes. The redistribution procedure described in $\S 4.3 .1$ takes care of the vertical gaps but it will not remedy the horizontal gaps.

Instead of doing a $2 \mathrm{D}$ version of the redistribution procedure, I choose to perform angular mashing to remedy the horizontal gap problem. Mashing is the 

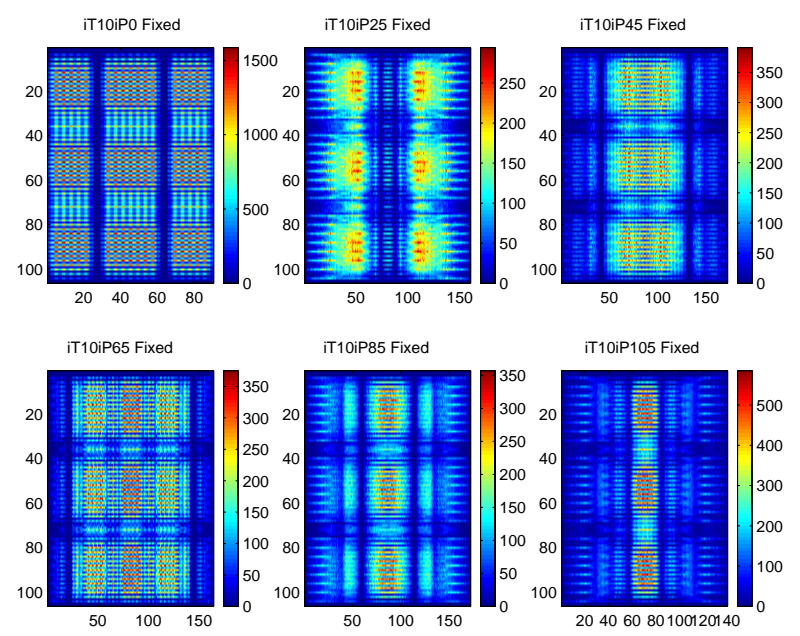

Figure 5.35: Redistributed Projections.

combination of projections taken at different angles and is used, for instance, with the LBL PET600 camera [77]. The primary reason for doing the mashing, instead of a $2 \mathrm{D}$ redistribution, is that redistribution is essentially a local smoothing of the data. In $2 \mathrm{D}$, nearest neighbor smoothing means that there are horizontal ZEB which will not be filled because all its adjacent bins have are also ZEBs. This implies that there will be planes that will not be populated. The forward projected value in these planes will remain zero and hence they will never be filled using the IRR algorithm.

For the mash, I combine the in-plane projections and the first off-plane projections prior to and after $\theta=\frac{\pi}{2}$. Fig. 5.34 shows the first row of Fig. 5.33 post-mashing. At this point, the redistribution procedure of $\S 4.3 .1$ can be used to take care of the horizontal ZEBs. Fig. 5.35 shows the same set of projections postredistribution. Note that the redistribution primarily affects the $\phi=0, \frac{\pi}{2}$ projections. The $\phi=\frac{\pi}{2}$ is not shown in this figure. The $2 \mathrm{D}$ normalization file may now be calculated as previously described.

At this point, I am essentially back in the $2 \mathrm{D}$ reconstruction mode. I follow 

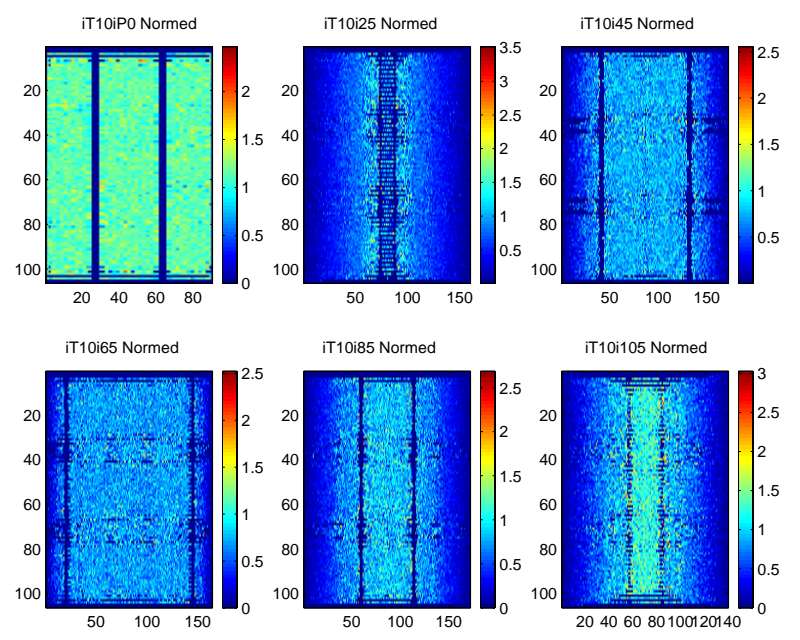

Figure 5.36: Normalized Projections.

the algorithm described in Chapter 4 in order to reconstruct the initial low-statistics estimate of the object. I use the IRR algorithm with the positivity constraint as prescribed and iterate until the object no longer changes. As an example, I reconstruct the $2 \mathrm{D}$ estimate for a $6.8 \times 10^{8}$ annihilations data set from a flood phantom using a $7.8 \times 10^{9}$ annihilation data set as the high-statistics data set to calculate the normalization file. Figures 5.36 and 5.37 show the normalized and filled projections after 20 iterations of IRR. The number of iterations before the projections cease to change is approximately 5. Fig. 5.38 shows the image sum of the squared pixel differences between the first iteration and the subsequent iterations.

In an attempt to increase the rate of convergence, I filled the ZEBs by using linear interpolation between the finite efficiency bins. The convergence rate did not change. It still took approximately 5 iterations before the projection data converged. The 20th iteration was the same whether the projection data was initially filled in using linear interpolation or not, suggesting that there is no need to do an initial estimate before performing the IRR algorithm. 

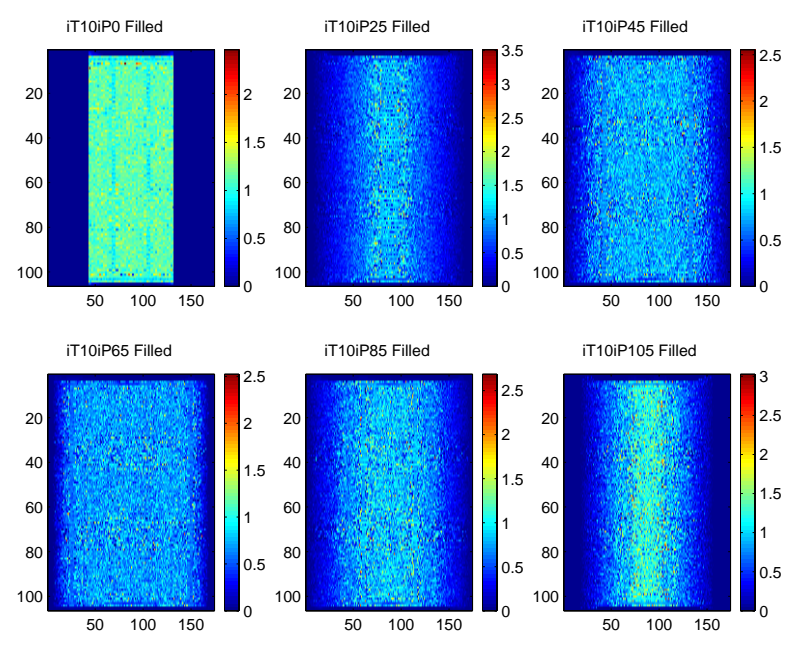

Figure 5.37: Filled Projections.

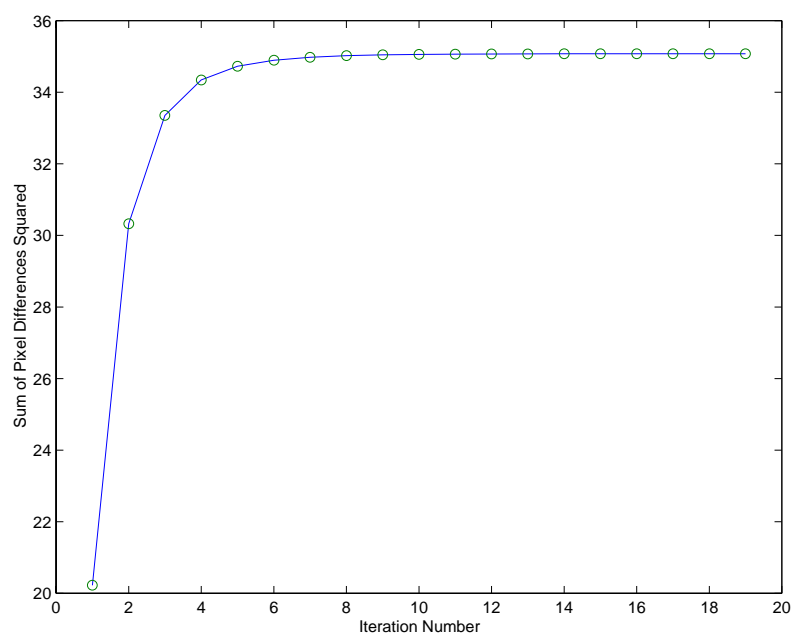

Figure 5.38: Sum of pixel differences between first and subsequent iterations for projection at $\phi=\frac{\pi}{2}$. The plot is zero suppressed and therefore does not show the first iteration. 

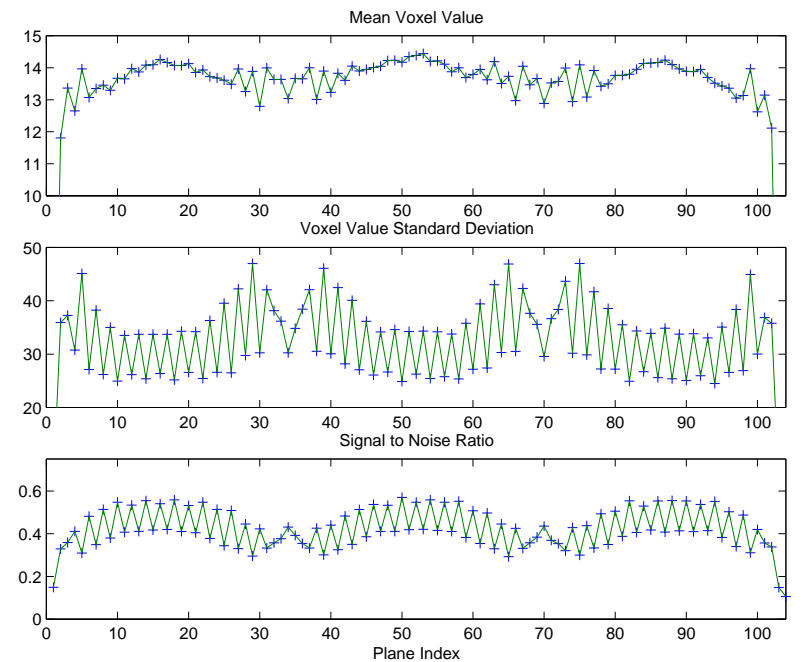

Figure 5.39: Mean voxel value and standard deviation of reconstructed flood source after the 5 th iteration of IRR.

Fig. 5.40 shows a few planes of the reconstructed flood source after the 5 th iteration while Fig. 5.39 shows the mean and standard deviation of the voxel values in each plane. The mean voxel value in the planes which are missing due to the gaps between modules are slightly underestimated. This is the same phenomenon that I have encountered with the ideal camera where the mean voxel values of the extreme planes are underestimated. Note that with the planes in the gaps exhibit characteristics of the previous extreme planes. Voxels in these planes have larger fluctuations from the mean. The overall large standard deviations compared to the mean values in all the planes are an evidence of the low number of events in the in-plane projections. These initial reconstructions have signal-to-noise ratios of approximately 0.5 .

The "sawtooth" behavior of the variance is the combined result of a) defining the interaction point to lie on the long axis of the crystal and b) mashing the first off-plane projections with the in-plane projections. Defining the interaction point to 

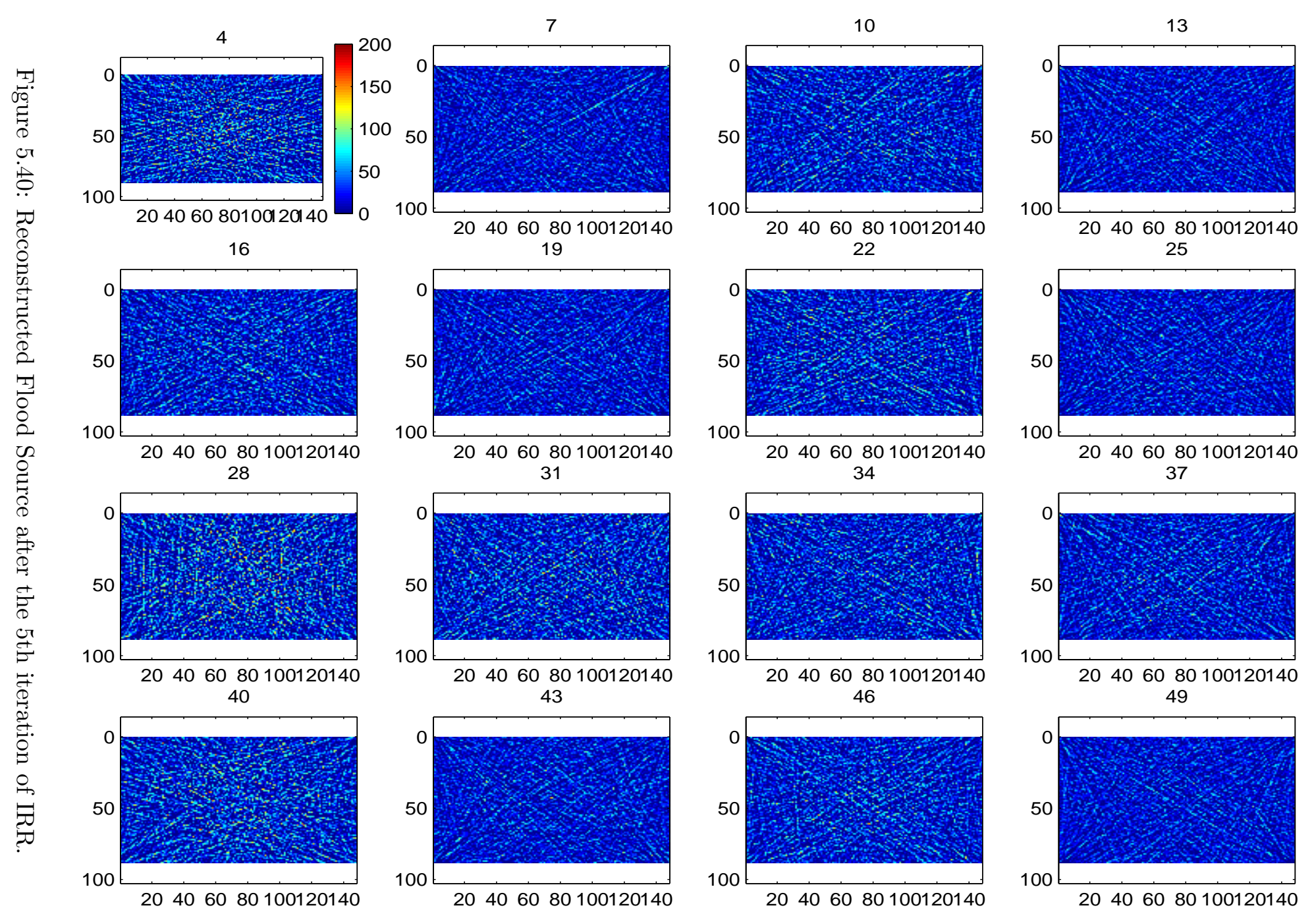
lie on the long axis of the crystal should leave three of every four planes in the inplane projection unpopulated. However, because the relatively large angular width of the in-plane projection (0.075 rad), annihilation events where both $511 \mathrm{keV}$ photons interact deep into the crystals can have chords with non-zero $\theta$ s small enough to be histogrammed in the in-plane projection. Thus, instead of having three of every four planes unpopulated, the first of every four planes is highly populated, the third is "fractionally" populated, and the second and fourth planes remain unpopulated. By mashing the first off-plane events with the in-plane events, these unpopulated bins become populated with fewer events than they really ought to have. However, this lower effective efficiency can be corrected by normalizing these bins which would have been unnormalizable without the mash. Normalizing the mashed data removes the "sawtooth" variation in the 0th moment, i.e. the mean, of the data, but not the variance.

\subsubsection{Final 3D Reconstruction}

The final reconstruction essentially follows the procedure already described in previous sections. The initial $2 \mathrm{D}$ reconstructed image is forward projected into a complete set of projections of the object to serve as an estimate for the truncated projections. However, since I have estimates of the complete data, I can redefine the ZEBs as truncated projection bins as well and treat them as any of the truly truncated projection bins. This leaves the original data as unmolested as possible.

There is yet another issue. Previously, in order to get the scale between the projected estimate and the true data, I summed over all the projection bins in the $\theta=\frac{\pi}{2}$ directions and compared them between the two projection sets. I did this between the normalized data and the projected estimate. I could do this because the in-plane events were a complete set, i.e. there were no missing information. In the limit of an infinite number of events, the normalized in-plane projections were the true projections of the object. This is no longer the case. In fact, normalizing the 


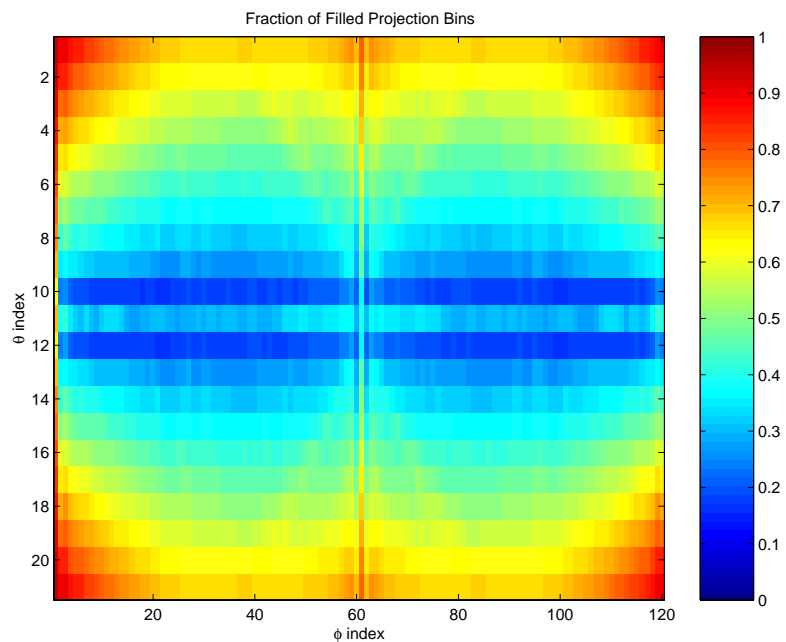

Figure 5.41: Fraction of filled bins in each projection plane. $\theta$-index 11 corresponds to $\theta=\frac{\pi}{2}$ and each index corresponds to a projection that has a polar angle that is 0.05 radians from the previous one. $\phi$-index 60 is $\phi=\frac{\pi}{2}$ and each index corresponds to a projection that is 0.025 radians from the previous one. This plot should be compared to the fraction of truncated bins in Fig. 5.6. 
data removes events from the high-efficiency projection bins without adding events to the ZEBs. This means that the number of events is not preserved, or that the normalized projections is no longer a true representation of the object.

In order to get around this issue, I "denormalize" the projected estimate with the same normalization set that I use for the true data. This would put the estimate in the same footing with the unnormalized data so that the total number of events in the data is the same as in the projected estimate. Once the scale is calculated, it is applied to the projected estimate. The truncated projection bins in the data are subsequently filled. The total fraction of bins that are filled either because of truncation or ZEBs is shown in Fig. 5.41. This figure should be compared to Fig. 5.6 which is the fraction of truncated bins in each projection. In particular, the $\theta=\frac{\pi}{2}$ projection has a higher fraction of filled bins than either the one before or after it. These are the result of the degenerate chords in the in-plane projections that are not present in the off-plane projections. Note that the same happens in the $\phi=0$

and $\frac{\pi}{2}$ projections. Once these projection bins are filled, the image is reconstructed according to the scheme discussed in the previous sections. The $3 \mathrm{D}$ reconstructed image of the flood will be shown in the following chapter.

\subsection{Summary of 3D Reconstruction Procedures}

The following is a list of the procedures that need to be done in order to successfully reconstruct 3D images using data from the PEM camera. Again, as in $\S 4.4, \mathrm{I}$ do not assume a specific aspect ratio for the PEM camera.

- Determine the angular widths of the projections near $\theta=\frac{\pi}{2}$. There maybe more than one projection for which a larger angular bin width is necessary to minimize ZEBs. If the aspect ratio of the camera is the same as I describe in this dissertation, then the in-plane and the first off-plane projections (there is one prior to and after $\theta=\frac{\pi}{2}$ ) need $0.075 \mathrm{rad}$ angular bin widths. The results of $\S 5.5$ indicate that $\delta \theta=0.05$ rad may be used for the remaining off-plane 
projections.

- Calculate normalization values for the reconstruction of the initial estimate of the object. Note that the exact same procedures that will be applied to the $3 \mathrm{D}$ projection data in order to get a $2 \mathrm{D}$ data set will need to be done to the high-statistics data set from which the normalization values will be calculated. For the purposes of this dissertation, I have used Monte Carlo data from a flood source as my high statistics data set. At the time of the writing of this dissertation, the preferred method of obtaining high statistics data for the real camera is to use a plane source within the FOV. The plane will contain the longest diagonal in the parallelepiped defined by the FOV.

- Calculate the normalization values for the 3D reconstruction of the final 3D object. Because all ZEBs (truncated projection bins, those that are due to the module gaps, and those that are due to the assignment of the interaction point to lie along the crystal axis) will be treated on the same footing, the 3D normalization values may be directly calculated from the ratio of the high statistics normalization phantom data and the phantom's known projections.

- Label all bins with normalization values above a threshold as ZEBs. The trehshold value may be determined by the procedure described in $\S 5.3$. Normalize the $3 \mathrm{D}$ projection data.

- Mash the in-plane data with the first off-plane projections until the ZEBs in the module gaps are populated. In this dissertation, the first off-plane projections were sufficient.

- Perform nearest- neighbor smoothing in each plane and reconstruct the 2D image in each plane as described in $§ 4.4$. Simulations indicate that 5 iterations of the IRR algorithm is sufficient. 
- Forward project the initial estimate into the polar projection angles that have $\delta \theta=0.05$. For the polar angles near $\theta=\frac{\pi}{2}$ that have $\delta \theta>.05$, let $\delta \theta_{i}^{\prime}=\frac{\delta \theta_{i}}{M}$ where $\mathrm{M}$ is the smallest positive integer that will satisfy $\delta \theta_{i}^{\prime}<\delta \theta_{i}$. Forward project the initial estimate into the new polar projection angles $\theta_{i j}^{\prime}=\theta_{i}-\frac{\delta \theta_{i}}{2}+$ $\left(j+\frac{1}{2}\right) \delta \theta_{i}^{\prime}, j=0 . .(M-1)$. Note that the projected data set is therefore larger than the true data set.

- Apply the inverse normalization to the projected data so that it may be scaled to the total number of events in the true data. Apply the calculated scaling factor to the projected data.

- Replace every non-ZEB projection bin value in the projected data set with the value of the corresponding projection bin in the true data set. Note that there are no true data that will correspond to the new projection angles $\theta_{i j}^{\prime}$, i.e. these forward projected data will be used in place of the true data near $\theta=\frac{\pi}{2}$.

- Use Colsher's algorithm to reconstruct the 3D object with the completed data set. Note that the projection data near $\theta=\frac{\pi}{2}$ will need to be multiplied further by a factor $\frac{\delta \theta_{i}^{\prime}}{0.05}<1$. Recall that 0.05 is the nominal polar angular bin width. 


\section{Chapter 6}

\section{Reconstructed Images}

To show the anticipated performance of both the camera and the reconstruction algorithms that I have discussed in this thesis, I now present reconstructed images of several different simulated phantoms. The simulation and reconstruction parameters are those discussed in previous chapters. In particular, the FOV has 104 planes in $z, 148$ slices in $x$ and 88 slices in $y$. Each voxel is $0.075 \mathrm{~cm}$ on a side.

\subsection{Flood Phantom}

The first phantom that I reconstructed is again the flood phantom. Fig. 6.2 is a $3 \mathrm{D}$ reconstruction of a flood phantom representing $4 \times 10^{8}$ annihilations, which translates to a $36 \times 10^{6}$ event data set. The images should be compared to those in Fig. 5.40 which are the $2 \mathrm{D}$ reconstructions after the 5 th iteration of IRR. The image statistics are shown in Fig. 6.3. Again, these plots should be compared to those in Fig. 5.39 which show the statistics of the $2 \mathrm{D}$ flood source reconstruction after the 5 th iteration of IRR.

The plot of the mean voxel values, if compared to the $2 \mathrm{D}$ reconstruction, is more characteristic of the reconstructed image for the ideal camera, slightly underestimated only at the extreme planes by a little less than $3 \%$. Some features due to the 
module gaps, such as the variation of the mean values in the gaps, are still apparent but are lower in relative magnitude (by up to a factor or 50\%) if compared to the $2 \mathrm{D}$ reconstruction. The noise in the gaps are also substantially reduced while the signal-to-noise ratio improves by as much as a factor of three in the middle planes.

As I discussed in $§ 5.7 .1$, the "sawtooth" behavior of the standard deviation is the combined result of a) defining the interaction point to lie on the long axis of the crystal and b) mashing the first off-plane projections with the in-plane projections. Including the other off-plane projections in the reconstruction reduces this effect tremendously but does not eliminate it. This result highlights the first weakness of the algorithms described in this dissertation, i.e. the noise structure of the reconstructed images will not be axially uniform. While it is true that the variation in the noise structure is small, it is nevertheless present.

\subsection{Point Sources}

\subsubsection{Profiles Phantom}

In order to measure the resolution of the camera, I simulate point sources along the borders of the upper top-right octant of the FOV and along the diagonal of the middle and the top planes. The locations and number designations of the point sources in the middle and top planes are shown in Fig. 6.4 (A). Three planes between the top and middle planes, namely planes 63,75 and 87 , have point sources at the corners of the octant. These sources represent a high-count acquisition of approximately $1.6 \times 10^{6}$ detected events per point source for 62 point sources (25 in the middle and top planes and four on three planes in between).

Figs. 6.5, 6.7 and 6.8 show the middle plane, the in-between planes, and the top plane of the reconstructed FOV respectively. Figs. 6.6 and 6.9 show the profiles through the point sources in the middle and top planes. "Profiles along Top $\mathrm{x}$ " means that the profile is the $x$ profile of the point sources along the top edge of 
the camera. Likewise, "Profiles along Right y" means that the profile is the $y$ profile of the point sources along the right edge of the camera.

To get the FWHM of these peaks, I fit them to one and two Gaussian models, taking the results from the better fit. If neither model is acceptable, the FWHM is calculated by up-sampling the distribution by 4 samples, linearly interpolating between the known samples, and looking for the two points to the left and right of the maximum value at which the distribution falls by a factor of two. The distance between these two points is reported as the FWHM. Tables 6.1, 6.2, and 6.3 summarize the results.

Generally, the reconstructed point sources can be easily discerned, exhibiting peak-to-valley ratios of up to 10:1. This is particularly true for the middle planes as can be seen in Fig. 6.5. The FWHM values of these reconstructed point sources are within the crystal width of $3.0 \mathrm{~mm}$ throughout the entire volume of the detector. In fact, the resolution is particularly good for a large fraction of the FOV, degrading to $3.0 \mathrm{~mm}$ only in regions close to the edges and corners, and very near to the openings of the camera.

The point sources, however, exhibit "star-like" artifacts that radiate in what I have called "Problem Regions" in the angular variable $\phi$, i.e. $\phi=0, \frac{\pi}{2}$. Again, these are primarily due to the lower angular-sampling rate in these directions. Comparing these reconstructed point sources with those that were reconstructed without 3D information, i.e. Fig. 4.15, it is curious that the "star-artifact" is not present in the 2D images. After many attempts trying to find out an explanation for the difference, it turns out that the difference lies in the display color scales that I used. In the former images, I used "grayscale" which corrects for the nonlinearities of human vision. This means that the gradations of the scale are finer in the middle of the scale than near the ends of the scale. On the other hand, I used the "linear-color" scale for the current images, i.e. the gradations in this scale is independent of the value in the scale. The $5 \%-10 \%$ artifacts are also present in the $2 \mathrm{D}$ image in spite. It is, however, consoling that the artifacts do not affect the FWHM resolution of the 
(A)

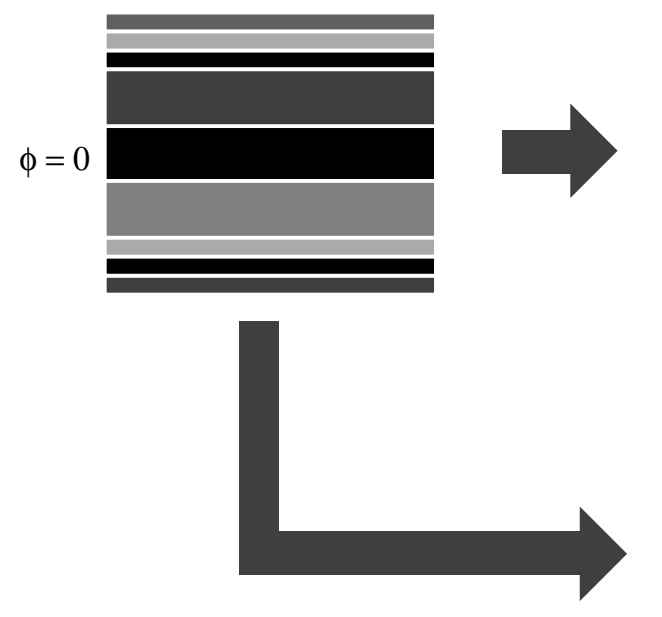

(B)
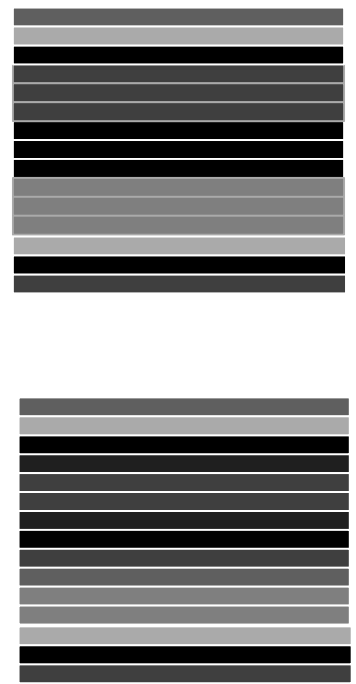

(C)

Figure 6.1: Pictorial representation of methods of splitting events in the problematic projection angles in polar angle $\phi$.

images and only slightly affect the isotropy of the full-width-at-tenth-max (FWTM ${ }^{1}$

In an attempt to minimize the extent of the artifacts, I studied an alternative way of redistributing the events that have polar angles near the problem regions. In particular, the method I have used, thus far, divides the events equally into all the angular bins that the wide and problematic angular bin is split. The effect of this is a step-wise discontinuity in the number of events. Figs. 6.1 (A) and (B) shows this method pictorially. Instead, I linearly interpolated between the the centers of these wide bins to generate a linear-discontinuous distribution. Figs. 6.1 (A) and (C) shows this method pictorially. The changes in the images that I observed were not significant. These artifacts represent the second weakness of the algorithms described in this dissertation.

\footnotetext{
${ }^{1}$ The FWTM is the width at which a distribution of a tenth of its maximum value.
} 


\subsubsection{Noise Phantom}

In order to compare the $2 \mathrm{D}$ reconstructions in $\S 4$ with the current $3 \mathrm{D}$ reconstruction procedures, I simulated point sources at the same locations as those in $\S 4.2 .3$ separated in $z$ by $0.6 \mathrm{~cm}$, i.e. two crystals or 8 reconstruction planes. The images represent $2 \times 10^{8}$ annihilations or $2 \times 10^{7}$ events in $12 \times 7=84$ point sources, i.e. $24 \times 10^{5}$ events per point source. Figs. 6.10 and 6.11 show the reconstructed point sources at the center and near the top of the FOV, respectively. The point sources are well reconstructed. To characterize these point sources, I took their "volumetric profiles" in the $x, y$, and $z$ axes. For each point source, I summed the voxels in two axes and calculated the FWHM of the resulting distribution in the third axis. Note that this is not a measure of the resolution of the point source, but rather a measure of the extent of the total activity in the point source. Table 6.4 shows the FWHM of the "volumetric profiles." A heading " $x$ " means that the point source was integrated in $y$ and $z$. I fitted the distributions to one and two Gaussian functions, and I took the results from the better fit. If neither models are acceptable, the FWHM is calculated by the up-sampling technique. Fig. 6.4(B) identifies the point sources in the table. Plane 53 is near the center plane while plane 93 is near the top of the FOV. All values are $\pm 0.75 \mathrm{~mm}$.

This study is similar to performing a Region-of-Interest study on the reconstructed point sources. Assuming a Gaussian PSF, the results indicate that the PEM camera is likely to obtain reconstructed point sources with $86 \%^{2}$ of integrated activity will be contained within approximately $2.5 \mathrm{~mm}$ of the location of the source. The average 3D FWHM of the point sources throughout the FOV is $2.5 \mathrm{~mm}$. Although I do not present quantitative results in this dissertation, it is encouraging that using the reconstruction procedures detailed in this dissertation, simulations show the PEM camera will be able to confine point source activity within less than a crystal width.

\footnotetext{
${ }^{2}$ The ratio of the integral $\int_{0}^{r_{o}} d r r^{2} \exp \left(\frac{-r^{2}}{2 \sigma^{2}}\right)$ evaluated for $r_{o}=F W H M$ and $r_{o}=\infty$ is 0.8627 .
} 
In order to characterize the noise in the reconstructed point sources, I generated five independent data sets which have only $5 \times 10^{7}$ annihilations. The variation in the peak values and integrated activity of the reconstructed sources should give a measure of the noise in the reconstruction. Table 6.5 summarizes the results of the study. The variation in the integrated activity and peak values of the point sources are between $0 \%-3 \%$.

\subsubsection{Derenzo-like Point Sources}

The Derenzo Phantom is a phantom designed to visually assess the resolving power of a camera. Cylindrical holes drilled through a block of material, often plexi glass, are filled with radioactivity and scanned with the camera being tested. Several regions have cylinders with different diameter holes whose spacing equal four times the diameter. Instead of simulating rods, I simulated point sources that were organized in a similar manner to a Derenzo Phantom. I filled the top right quadrant of the central plane with point sources that were $1.0 \mathrm{~mm}$ in diameter with $4.0 \mathrm{~mm}$ center-to-center spacing. The top left, bottom left and bottom right quadrants were filled with point sources that had $1.25 \mathrm{~mm}, 1.5 \mathrm{~mm}$, and $2.0 \mathrm{~mm}$ diameter point sources respectively. This pattern was repeated every $8.0 \mathrm{~mm}$ in $z$. Figs. 6.12 and 6.13 show the planes near the center and top of the FOV respectively. Note that in the middle plane of the FOV, the point sources can be easily distinguished from each other, even the small sources that are some distance from the center of the plane. However, the same sources are difficult to resolve near the top of the camera. Only the larger sources close to the center of the plane are easily recognizable.

The modified Derenzo Phantom that I used is a good tool to evaluate the performance of the reconstruction algorithms that I have discussed. It shows the variation in the quality of the reconstructed point sources throughout the entire FOV. Again, it is encouraging to consider that using the algorithms I have discussed, the PEM camera will be able to detect aggregations of radioactivity as small as $3.00 \mathrm{~mm}$, 
regardless of the location in the FOV. Current PET systems that are optimized for breast cancer imaging are able to image structures of the order of $1 \mathrm{~cm}$ in size.

\subsection{Extended Source Phantoms}

\subsubsection{Defrise Phantom}

The Defrise Phantom is a phantom that is composed of alternating planes of constant activity and no activity. It is designed to investigate how activity can "leak" from one plane to another in 3D reconstruction. This leakage is common in $3 \mathrm{D}$ reconstruction algorithms and usually increases as one comes closer to the edge of the FOV. I simulated such a phantom in the lower half of the PEM FOV. The lower top-left, lower bottom-left and the lower bottom-right octants are divided into twenty-four $0.15 \mathrm{~cm}$ planes, twelve $0.30 \mathrm{~cm}$ planes, and six $0.60 \mathrm{~cm}$ planes of alternating constant and zero activity respectively. Figs. 6.14 and 6.15 are the $x$ and $y$ slices of the reconstructed image respectively. Both images display planes from the most negative $x$ and $y$ values, i.e. " $x$ Slice 1" is the left most $x$ slice and " $y$ Slice 1" is the bottom most $y$ slice. Fig. 6.16 shows the mean voxel value and voxel standard deviation of a $3 \times 3$ voxel ROI located at the center of each quadrant in each plane. Fig. 6.17 shows the same information but for a $15 \times 15$ voxel ROI. The individual planes can be seen in the quadrants in which the planes with activity are $0.3 \mathrm{~cm}$ and $0.6 \mathrm{~cm}$ thick. The half-crystal-thick planes cannot be discerned. This is not too surprising. The bars of a true Defrise phantom are much coarser than the resolution of the camera.

\subsubsection{Spheres and Ellipsoids}

The final phantom that I simulated are extended sources of spheres and ellipsoids. While it is true that because both the acquisition and reconstruction processes are linear, we need only to simulate point sources in order to characterize 
the camera. But its always fun to see how well you can see other objects in it! There are four objects in the FOV in this phantom.

- A sphere of $R=0.75 \mathrm{~cm}$ is located near the central plane of the FOV and lies along the $x$ axis displaced by a small distance from the $y$ axis.

- An ellipsoid is located at the center of the $x y$ plane but displaced by a quarter of the camera's axial extent in $-z$. Its principal axes have dimensions $(2.75$, $1.0,1.0) \mathrm{cm}$.

- Another ellipsoid is located near the top quarter of the FOV, oriented along the $y$ axis but displaced from it to the right. Its principal axes have dimensions $(1.0,3.0,1.0) \mathrm{cm}$.

- Finally, a long ellipsoid extending through the entire axial extent of the camera is located on the $y$ axis displaced by a small distance from the $x$ axis. Its principal axes have dimensions $(0.75,0.75,3.0) \mathrm{cm}$.

The ellipsoids have twice as many annihilations as there are in the sphere so that with volumes equal to $(1.7671,11.5192,12.5664,7.0686) \mathrm{cm}^{3}$ respectively ${ }^{3}$, their relative activities are expected to be $1.0000: 0.3068: 0.2812: 0.5000$. All the objects are reconstructed well with $10^{9}$ annihilations or $9 \times 10^{7}$ detected events. The mean voxel values in each of these objects are $(1.1396,0.3591,0.3359,0.5711) \times 10^{5}$ respectively, i.e. the measured relative activites are $1.0000: 0.3151: 0.2948: 0.5011$. I performed the same reconstruction with half this number of events and the objects were still clearly reconstructed.

The number of events that I simulated is consistent with the anticipated number of events that will be detected during a PEM scan. The anticipated FDG dose is $1.0 \mathrm{mCi}$ which is equivalent to $3.7 \times 10^{7} \mathrm{~Bq}$. The ratio of the volume of the FOV to the total volume of a typical $140 \mathrm{lbs}$. human female is approximately 1\%,

\footnotetext{
${ }^{3}$ The volume of an ellipsoid is $\frac{4}{3} \pi r_{1} r_{2} r_{3}$.
} 
and thus, some $3.7 \times 10^{5}$ annihilations per second can be expected to occur within the PEM camera. For a 20 minute scan, this translates to approximately $0.45 \times 10^{9}$ annihilations, consistent with the number of events that I have generated. 


\begin{tabular}{cccc}
\hline \hline & $x$ & $y$ & $z$ \\
\hline Plane 51 Source 1 & $1.54 \pm 0.75 \mathrm{~mm}$ & $1.56 \mathrm{~mm}$ & $1.54 \mathrm{~mm}$ \\
\hline Plane 51 Source 2 & 1.50 & 1.59 & 1.59 \\
\hline Plane 51 Source 3 & 2.05 & 2.07 & 2.05 \\
\hline Plane 51 Source 4 & 2.54 & 2.33 & 2.52 \\
\hline Plane 51 Source 5 & 2.63 & 2.43 & 2.43 \\
\hline Plane 51 Source 6 & 2.90 & 2.09 & 2.43 \\
\hline Plane 51 Source 7 & 2.62 & 2.25 & 2.48 \\
\hline Plane 51 Source 8 & 2.63 & 2.31 & 2.40 \\
\hline Plane 51 Source 9 & 2.80 & 2.40 & 2.63 \\
\hline Plane 51 Source 10 & 2.72 & 3.11 & 2.57 \\
\hline Plane 51 Source 11 & 3.01 & 3.21 & 2.81 \\
\hline Plane 51 Source 12 & 2.75 & 2.73 & 2.84 \\
\hline Plane 51 Source 13 & 2.20 & 3.16 & 2.25 \\
\hline Plane 51 Source 14 & 2.40 & 3.17 & 2.26 \\
\hline Plane 51 Source 15 & - & - & - \\
\hline Plane 51 Source 16 & 1.53 & 2.99 & 2.15 \\
\hline Plane 51 Source 17 & 1.49 & 2.83 & 2.35 \\
\hline Plane 51 Source 18 & 1.52 & 2.25 & 1.52 \\
\hline Plane 51 Source 19 & 1.59 & 2.09 & 1.51 \\
\hline Plane 51 Source 20 & 1.51 & 1.92 & 2.06 \\
\hline Plane 51 Source 21 & 1.59 & 1.67 & 1.68 \\
\hline Plane 51 Source 22 & 2.09 & 1.34 & 1.83 \\
\hline Plane 51 Source 23 & 2.14 & 2.62 & 2.24 \\
\hline Plane 51 Source 24 & 2.54 & 2.53 & 2.43 \\
\hline Plane 51 Source 25 & 2.83 & 3.05 & 2.53 \\
\hline & & &
\end{tabular}

Table 6.1: FWHM of point sources in the middle plane of the FOV in Profiles Phantom. 


\begin{tabular}{lccc}
\hline \hline & $x$ & $y$ & $z$ \\
\hline Plane 63 Source 1 & $1.53 \pm 0.75 \mathrm{~mm}$ & $1.56 \mathrm{~mm}$ & $1.99 \mathrm{~mm}$ \\
\hline Plane 63 Source 2 & 3.03 & 1.84 & 1.97 \\
\hline Plane 63 Source 3 & - & - & - \\
\hline Plane 63 Source 4 & 2.15 & 2.96 & 2.45 \\
\hline Plane 75 Source 1 & 1.59 & 1.61 & 2.06 \\
\hline Plane 75 Source 2 & 2.91 & 2.19 & 2.57 \\
\hline Plane 75 Source 3 & - & - & - \\
\hline Plane 75 Source 4 & 1.73 & 2.81 & 2.43 \\
\hline Plane 87 Source 1 & 1.65 & 1.86 & 1.73 \\
\hline Plane 87 Source 2 & 3.04 & 2.22 & 2.61 \\
\hline Plane 87 Source 3 & - & - & - \\
\hline Plane 87 Source 4 & 2.51 & 2.90 & 2.49 \\
\hline
\end{tabular}

Table 6.2: FWHM of point sources in planes between the middle and top planes of Profiles Phantom. 


\begin{tabular}{|c|c|c|c|}
\hline & $x$ & $y$ & $z$ \\
\hline Plane 99 Source 1 & $1.91 \pm 0.75 \mathrm{~mm}$ & $2.55 \mathrm{~mm}$ & $2.05 \mathrm{~mm}$ \\
\hline Plane 99 Source 2 & 2.65 & 2.43 & 2.65 \\
\hline Plane 99 Source 3 & 2.84 & 2.81 & 2.80 \\
\hline Plane 99 Source 4 & 2.86 & 2.87 & 2.75 \\
\hline Plane 99 Source 5 & 3.01 & 2.91 & 2.90 \\
\hline Plane 99 Source 6 & 3.21 & 2.72 & 2.64 \\
\hline Plane 99 Source 8 & 3.11 & 2.69 & 2.78 \\
\hline Plane 99 Source 9 & 3.09 & 2.73 & 2.81 \\
\hline Plane 99 Source 10 & 3.21 & 2.66 & 2.93 \\
\hline Plane 99 Source 11 & 3.18 & 2.68 & 3.21 \\
\hline Plane 99 Source 12 & 2.85 & 2.93 & 3.01 \\
\hline Plane 99 Source 13 & 2.71 & 2.86 & 2.85 \\
\hline Plane 99 Source 14 & 2.63 & 2.72 & 2.72 \\
\hline Plane 99 Source 15 & - & - & - \\
\hline Plane 99 Source 16 & 2.01 & 2.85 & 2.41 \\
\hline Plane 99 Source 17 & 2.13 & 2.79 & 2.33 \\
\hline Plane 99 Source 18 & 2.22 & 2.55 & 2.27 \\
\hline Plane 99 Source 19 & 2.31 & 2.31 & 2.15 \\
\hline Plane 99 Source 20 & 2.55 & 2.25 & 2.09 \\
\hline Plane 99 Source 21 & 2.09 & 2.09 & 2.20 \\
\hline Plane 99 Source 22 & 2.42 & 2.41 & 2.41 \\
\hline Plane 99 Source 23 & 2.45 & 2.53 & 2.45 \\
\hline Plane 99 Source 24 & 2.62 & 2.75 & 2.53 \\
\hline Plane 99 Source 25 & 2.53 & 2.66 & 2.46 \\
\hline
\end{tabular}

Table 6.3: FWHM of point sources near the top plane of the FOV of Profiles Phantom. 


\begin{tabular}{lccc}
\hline \hline & $x$ & $y$ & $z$ \\
\hline Plane 53 Source 1 & $1.79 \pm 0.75 \mathrm{~mm}$ & $1.88 \mathrm{~mm}$ & $2.06 \mathrm{~mm}$ \\
\hline Plane 53 Source 2 & 1.92 & 1.92 & 2.25 \\
\hline Plane 53 Source 3 & 1.88 & 1.87 & 2.25 \\
\hline Plane 53 Source 4 & 2.68 & 2.74 & 2.98 \\
\hline Plane 53 Source 5 & 2.73 & 2.85 & 3.27 \\
\hline Plane 53 Source 6 & 2.63 & 2.25 & 3.01 \\
\hline Plane 53 Source 7 & 3.37 & 3.37 & 3.21 \\
\hline Plane 93 Source 1 & 1.90 & 2.00 & 1.93 \\
\hline Plane 93 Source 2 & 2.10 & 2.10 & 2.25 \\
\hline Plane 93 Source 3 & 2.10 & 2.10 & 2.43 \\
\hline Plane 93 Source 4 & 2.60 & 2.80 & 3.15 \\
\hline Plane 93 Source 5 & 2.50 & 2.65 & 2.99 \\
\hline Plane 93 Source 6 & 2.90 & 2.25 & 3.00 \\
\hline Plane 93 Source 7 & 3.18 & 3.14 & 3.35 \\
\hline
\end{tabular}

Table 6.4: Volumetric Extent of the reconstructed point sources in the Noise Phantom.

\begin{tabular}{lcccc}
\hline \hline & Integrated Activity $\dagger$ & \% Variation & Peak Value $\dagger$ & $\%$ Variation \\
\hline Plane 53 Source 1 & $5.7453 \pm 0.0107$ & 0.1868 & $0.1379 \pm 0.0016$ & 1.2027 \\
\hline Plane 53 Source 2 & $4.8639 \pm 0.0111$ & 0.2289 & $0.0903 \pm 0.0008$ & 0.8860 \\
\hline Plane 53 Source 3 & $5.3688 \pm 0.0229$ & 0.4264 & $0.0932 \pm 0.0007$ & 0.7035 \\
\hline Plane 53 Source 4 & $5.0560 \pm 0.0843$ & 1.6677 & $0.0652 \pm 0.0013$ & 1.9743 \\
\hline Plane 53 Source 5 & $4.0320 \pm 0.0763$ & 1.8915 & $0.0516 \pm 0.0007$ & 1.4018 \\
\hline Plane 53 Source 6 & $4.8363 \pm 0.0097$ & 0.2003 & $0.0513 \pm 0.0011$ & 2.0908 \\
\hline Plane 53 Source 7 & $5.0800 \pm 0.0903$ & 1.7779 & $0.0429 \pm 0.0008$ & 1.8617 \\
\hline Plane 93 Source 1 & $6.2568 \pm 0.1221$ & 1.9516 & $0.1265 \pm 0.0010$ & 0.7637 \\
\hline Plane 93 Source 2 & $5.5635 \pm 0.0256$ & 0.4611 & $0.0889 \pm 0.0012$ & 1.3508 \\
\hline Plane 93 Source 3 & $5.5116 \pm 0.0587$ & 1.0662 & $0.0833 \pm 0.0110$ & 1.3254 \\
\hline Plane 93 Source 4 & $5.1003 \pm 0.0358$ & 0.7025 & $0.0511 \pm 0.0181$ & 3.5425 \\
\hline Plane 93 Source 5 & $4.7882 \pm 0.0572$ & 1.1952 & $0.0444 \pm 0.0051$ & 1.1490 \\
\hline Plane 93 Source 6 & $4.2605 \pm 0.0421$ & 0.9877 & $0.0425 \pm 0.0011$ & 2.5543 \\
\hline Plane 93 Source 7 & $4.7482 \pm 0.0722$ & 1.5217 & $0.0375 \pm 0.0006$ & 1.6085 \\
\hline
\end{tabular}

Table 6.5: Variation in integrated point source activity and peak values of point sources in Noise Phantom. † Units are arbitrary. 


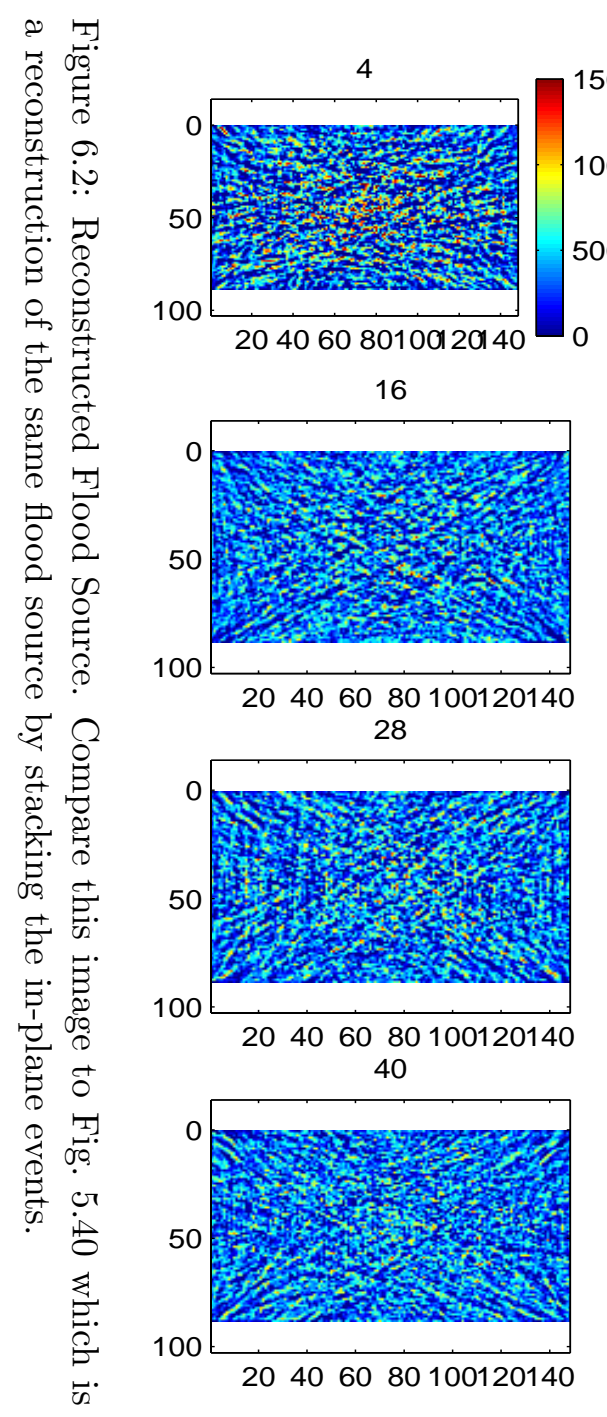

7

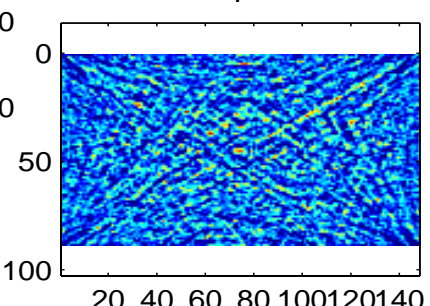

20406080100120140

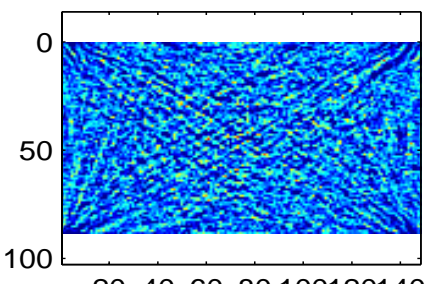

$204060 \quad 80100120140$ 31

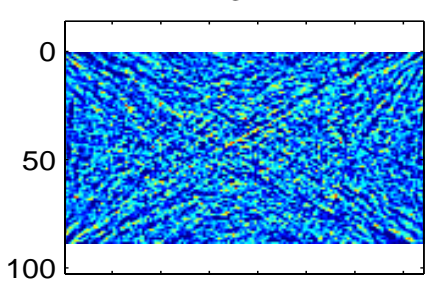

20406080100120140 43

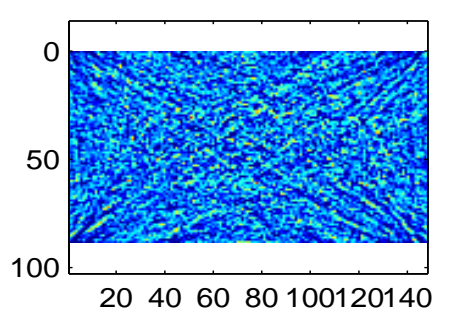

10
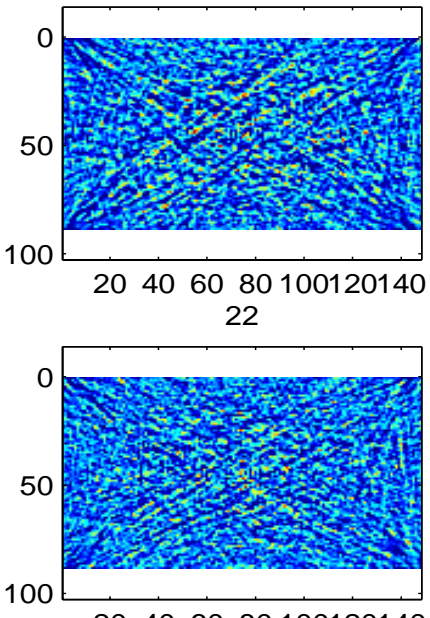
20406080100120140 34

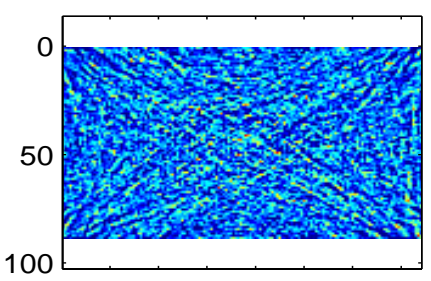

20406080100120140 46

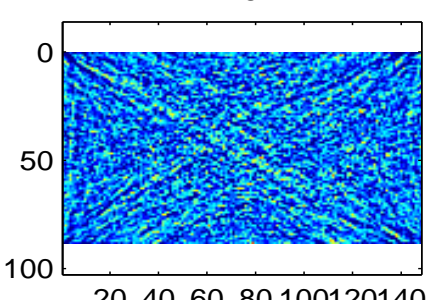

20406080100120140
13

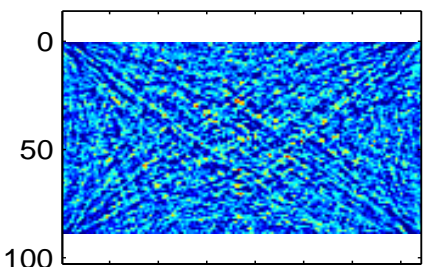

20406080100120140
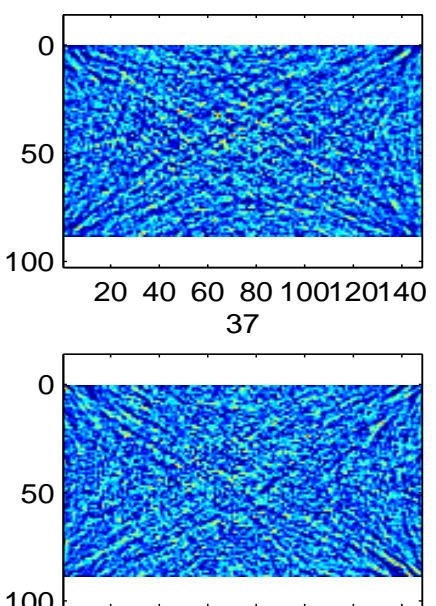

20406080100120140 49

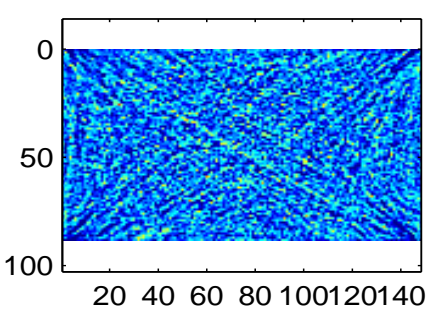



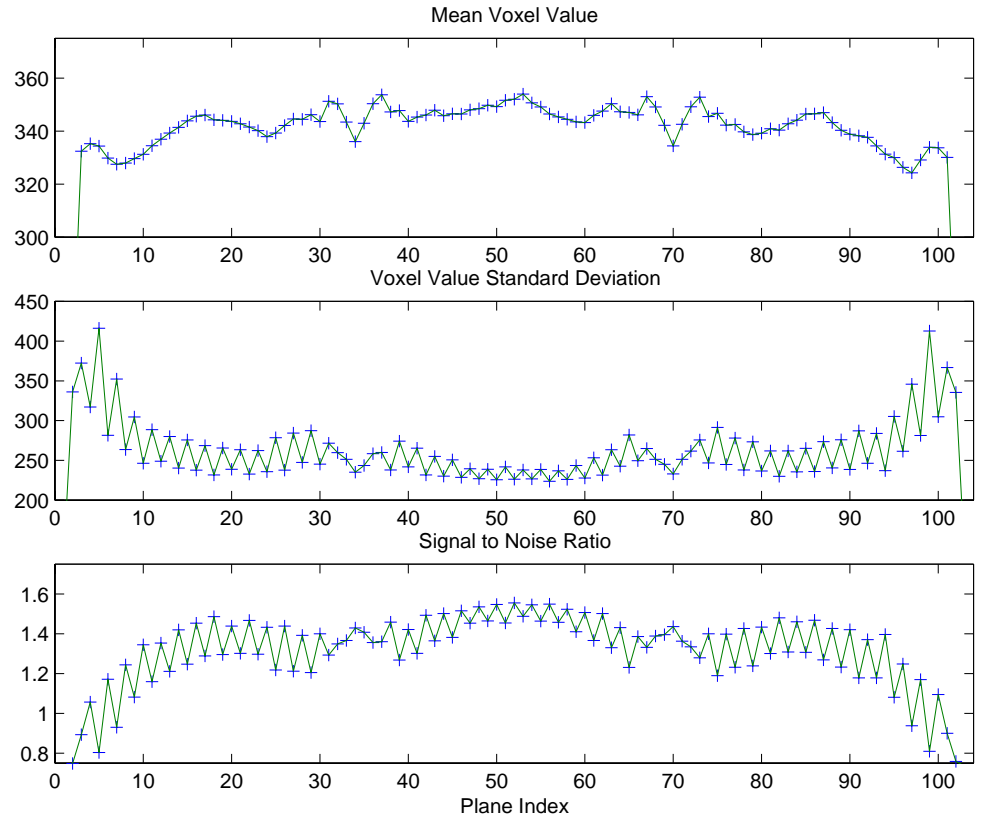

Figure 6.3: Image statistics of Flood Phantom.

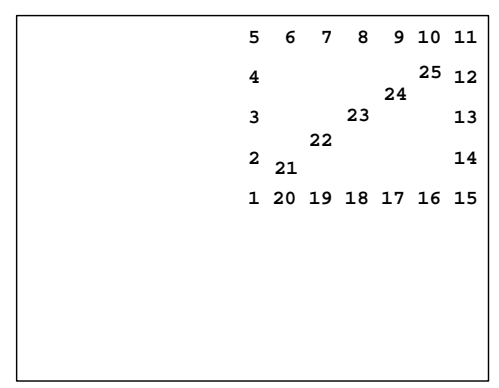

(A)

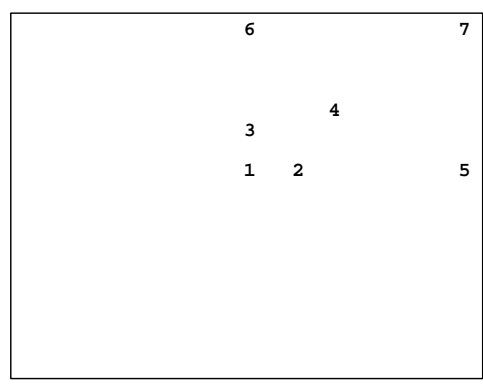

(B)

Figure 6.4: Point Sources Identification Numbers. (A) was simulated to measure profiles through the point sources in $\S 6.2 .1$ while (B) was simulated to study the noise characteristics of point sources in $\S 6.2 .2$. 

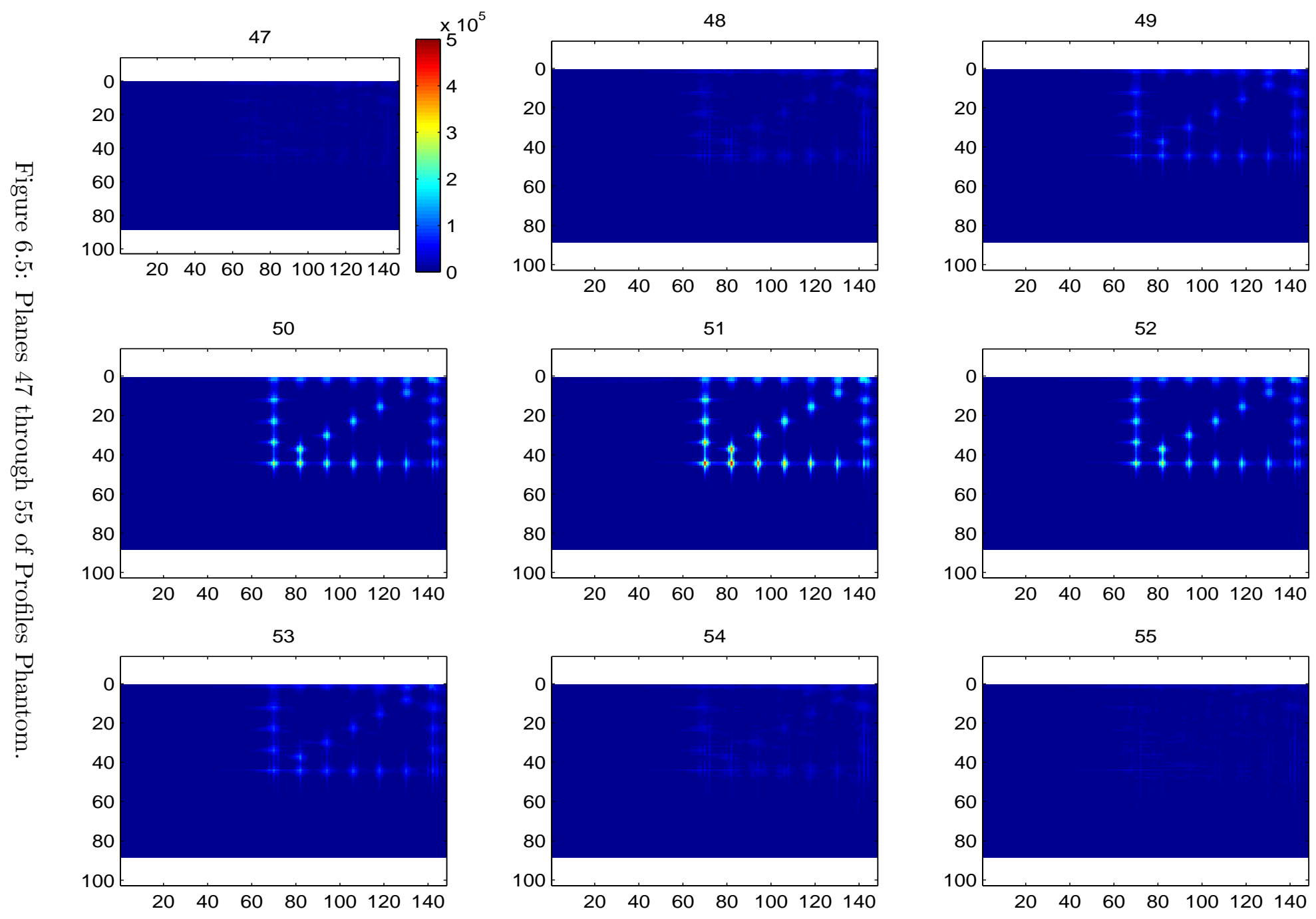

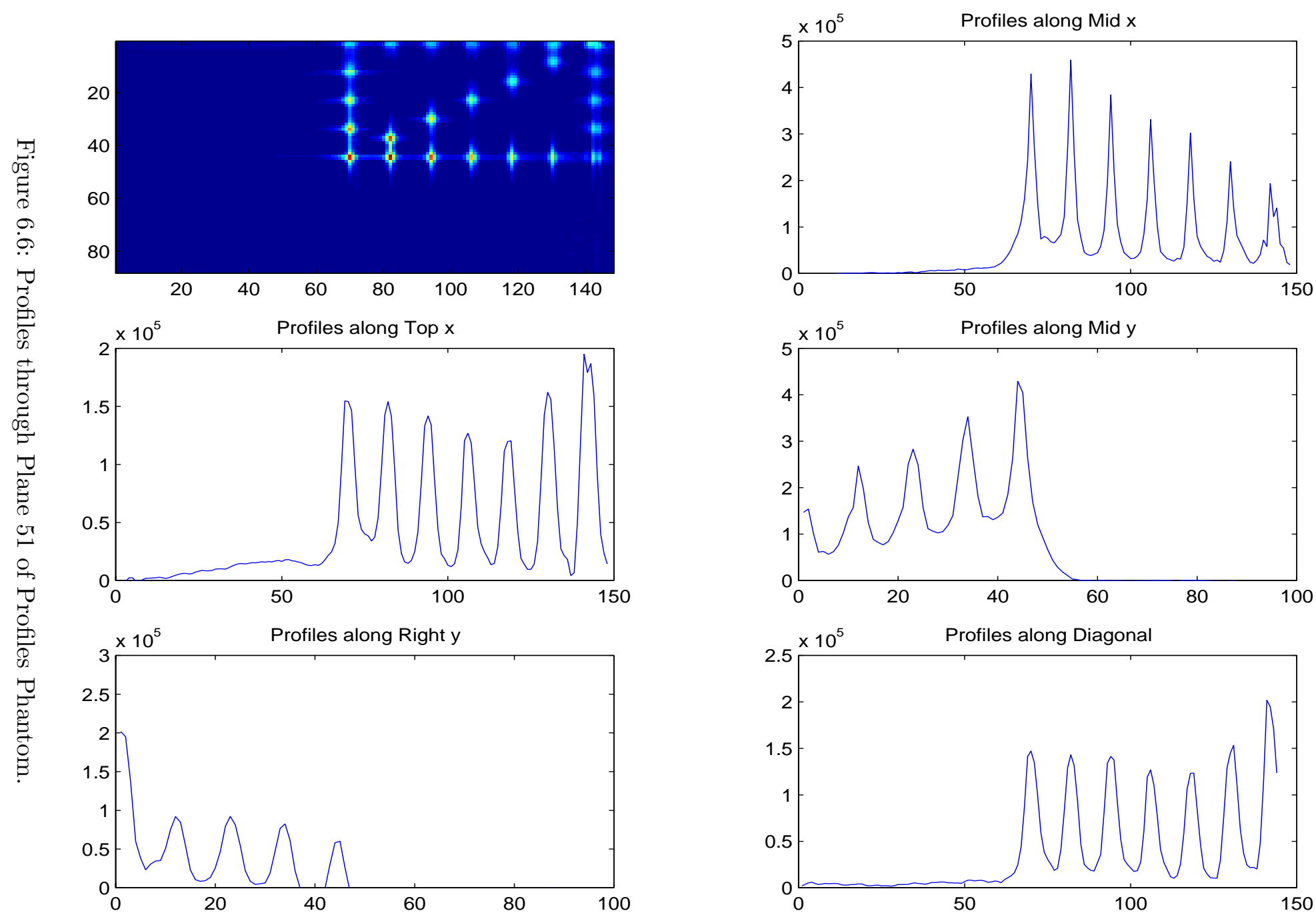

总 

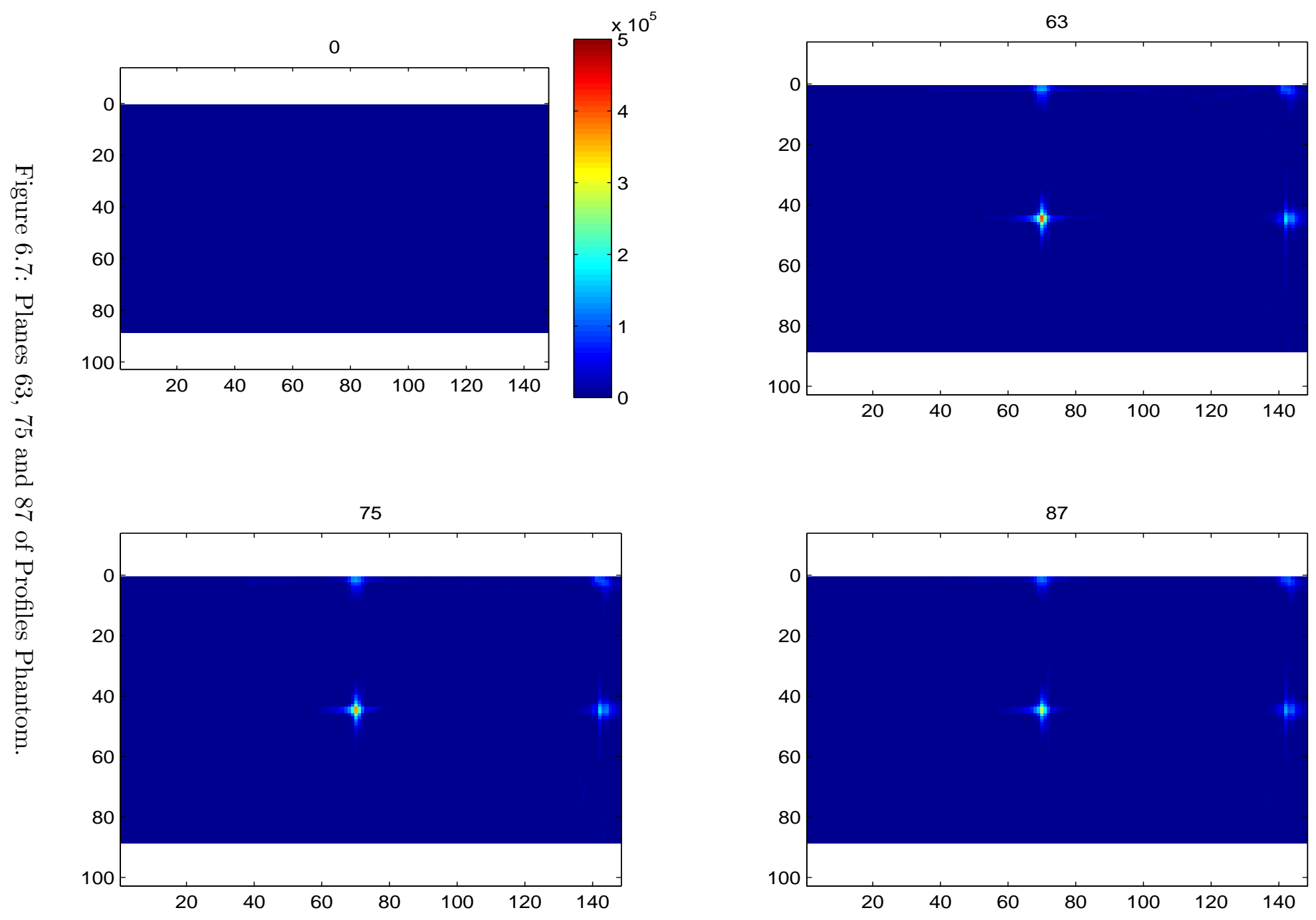

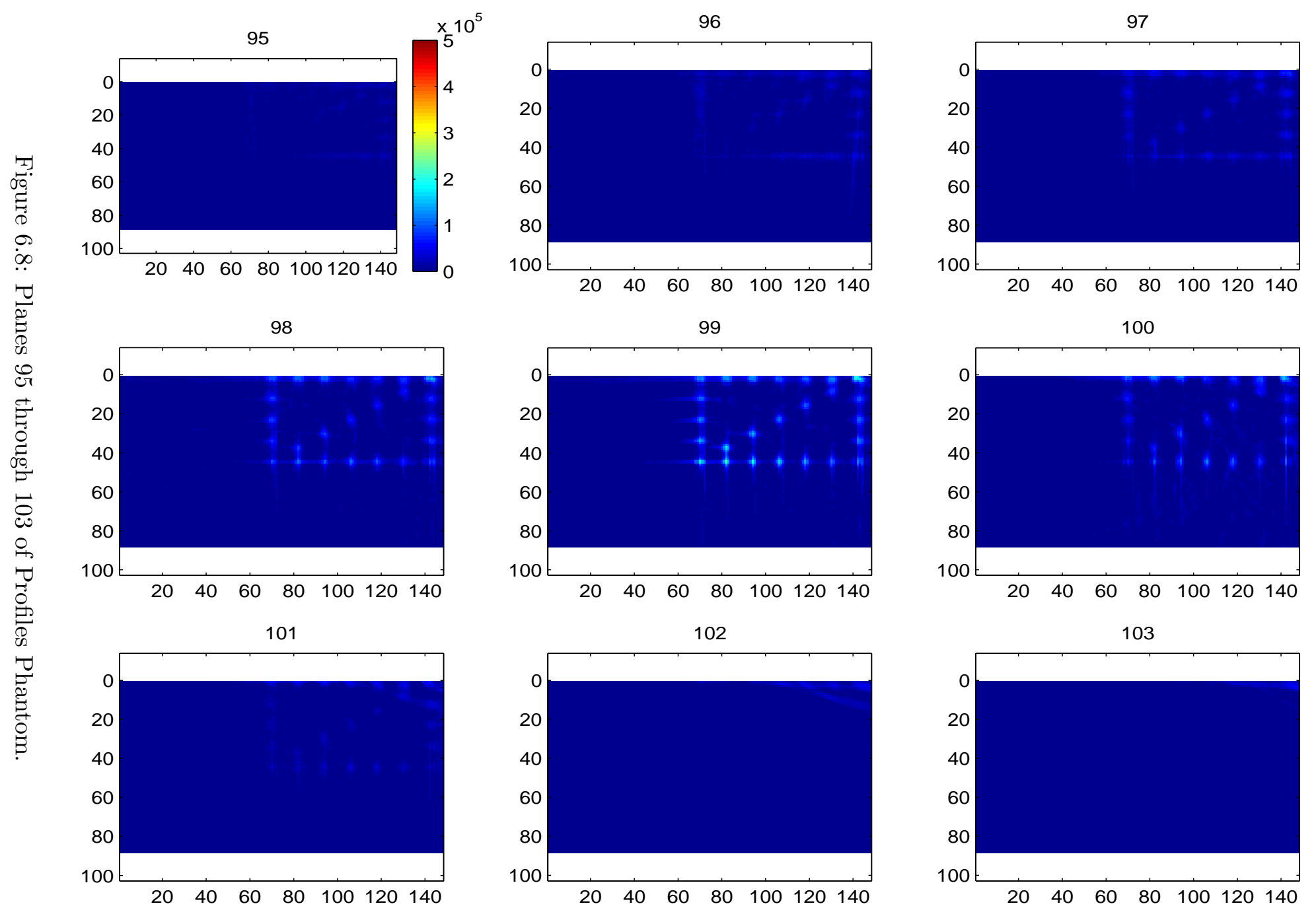

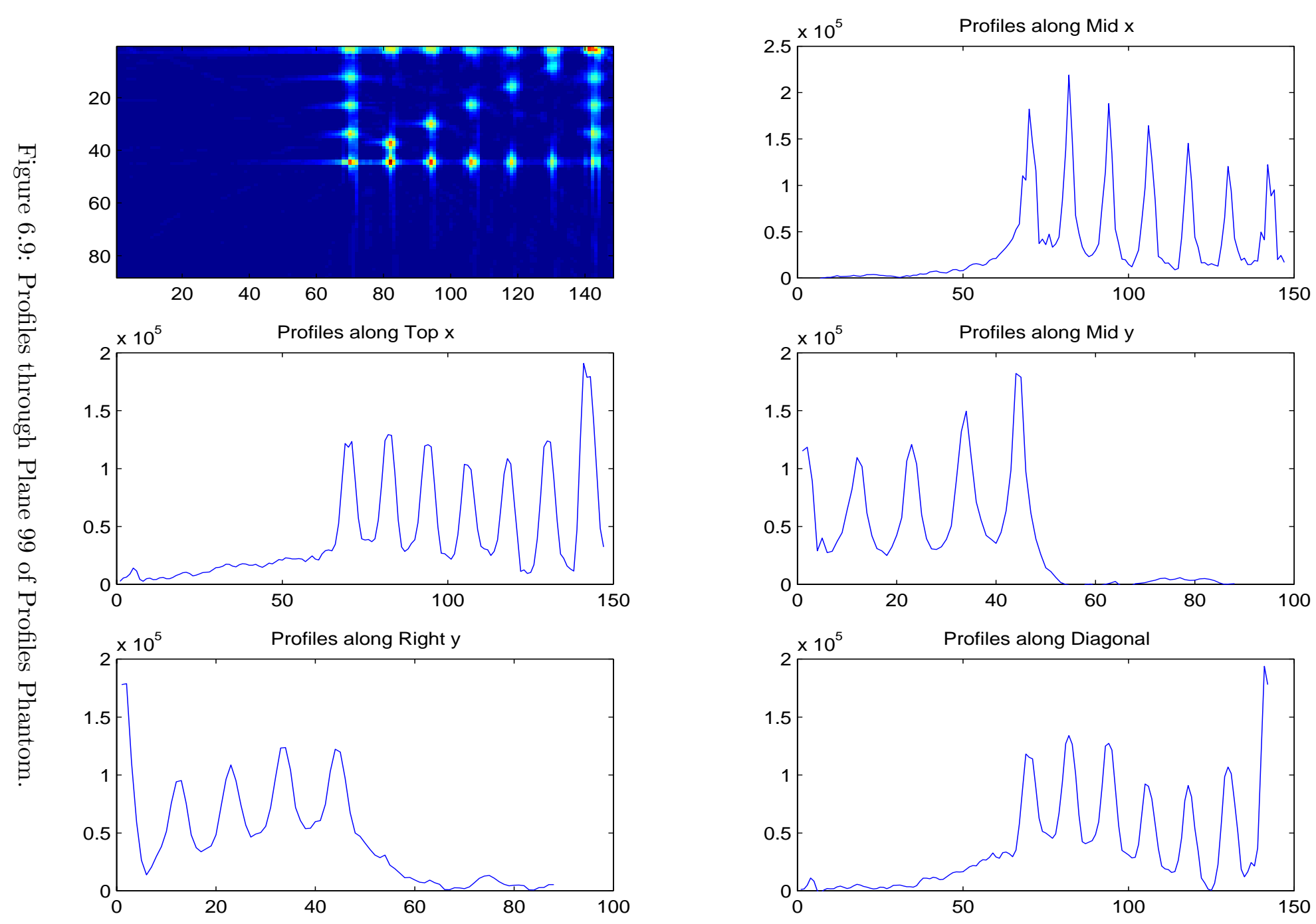

总 

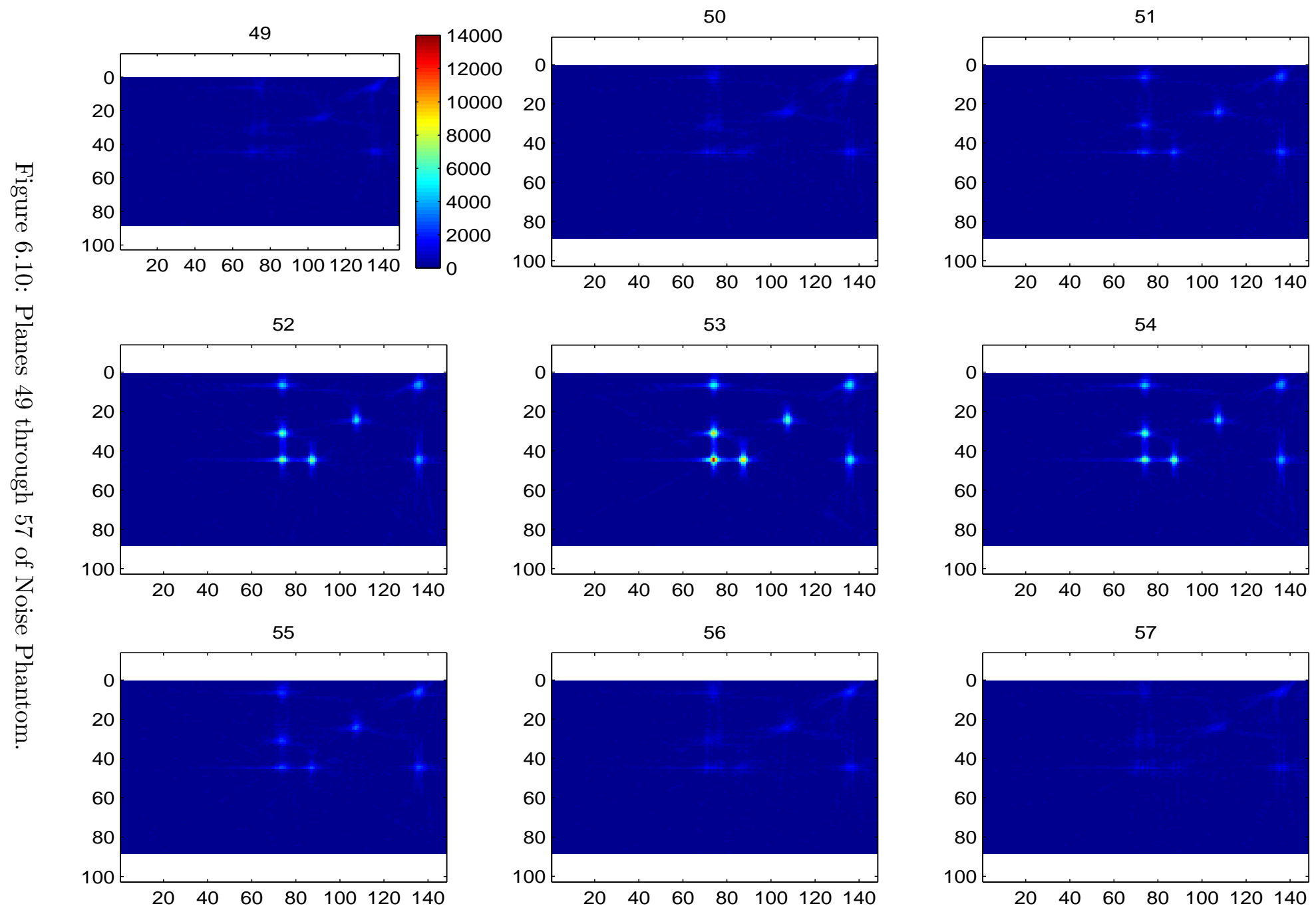

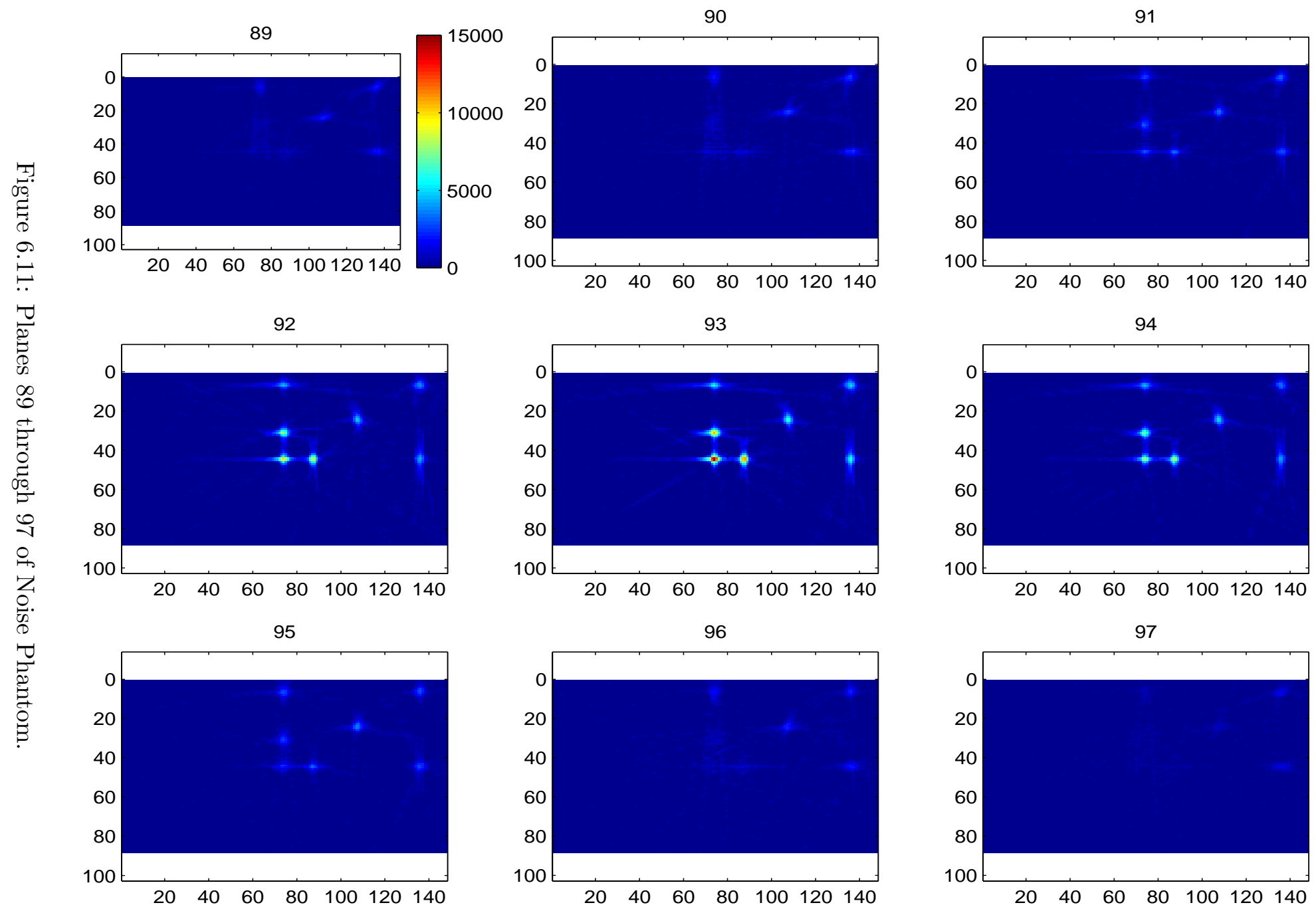

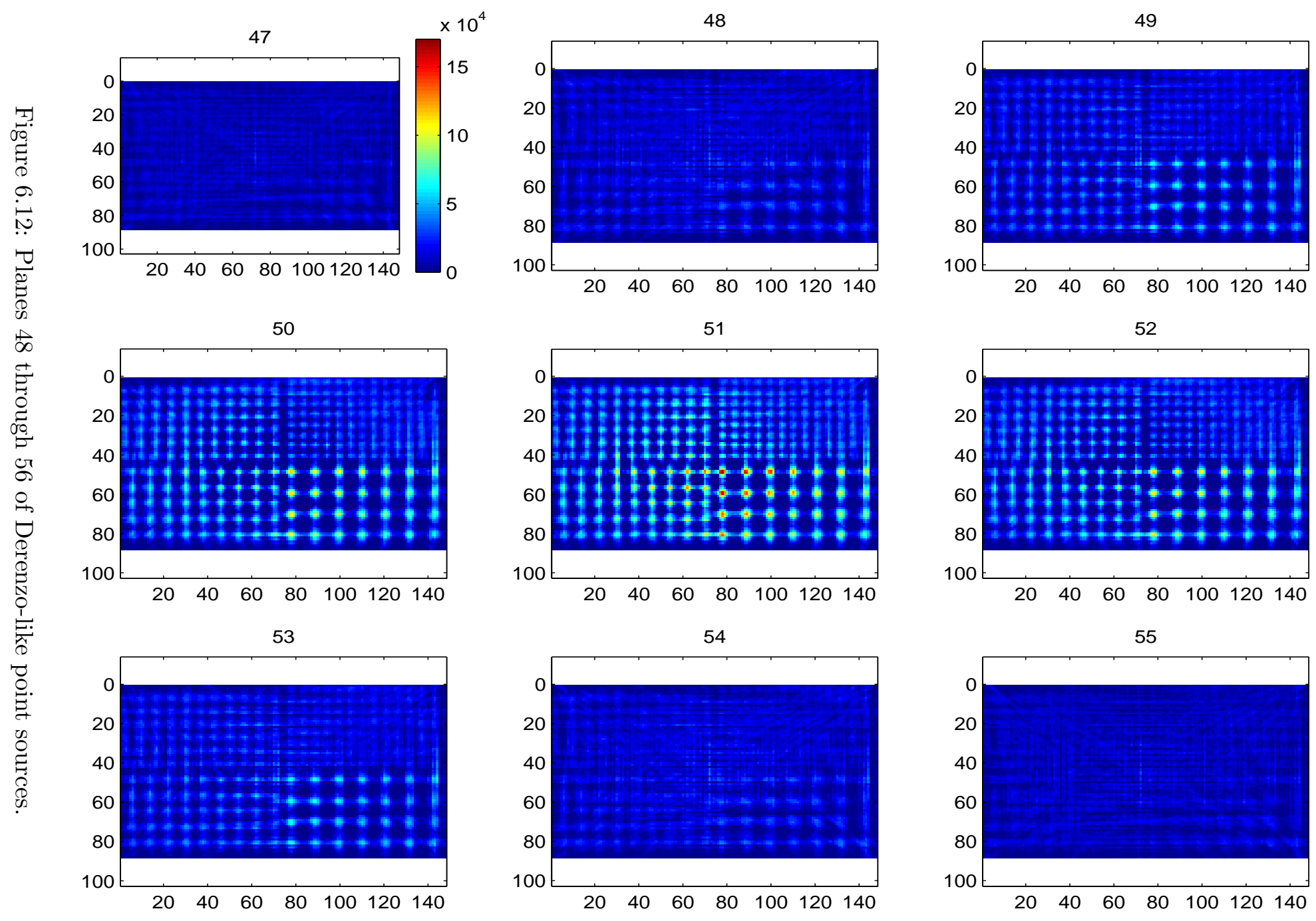

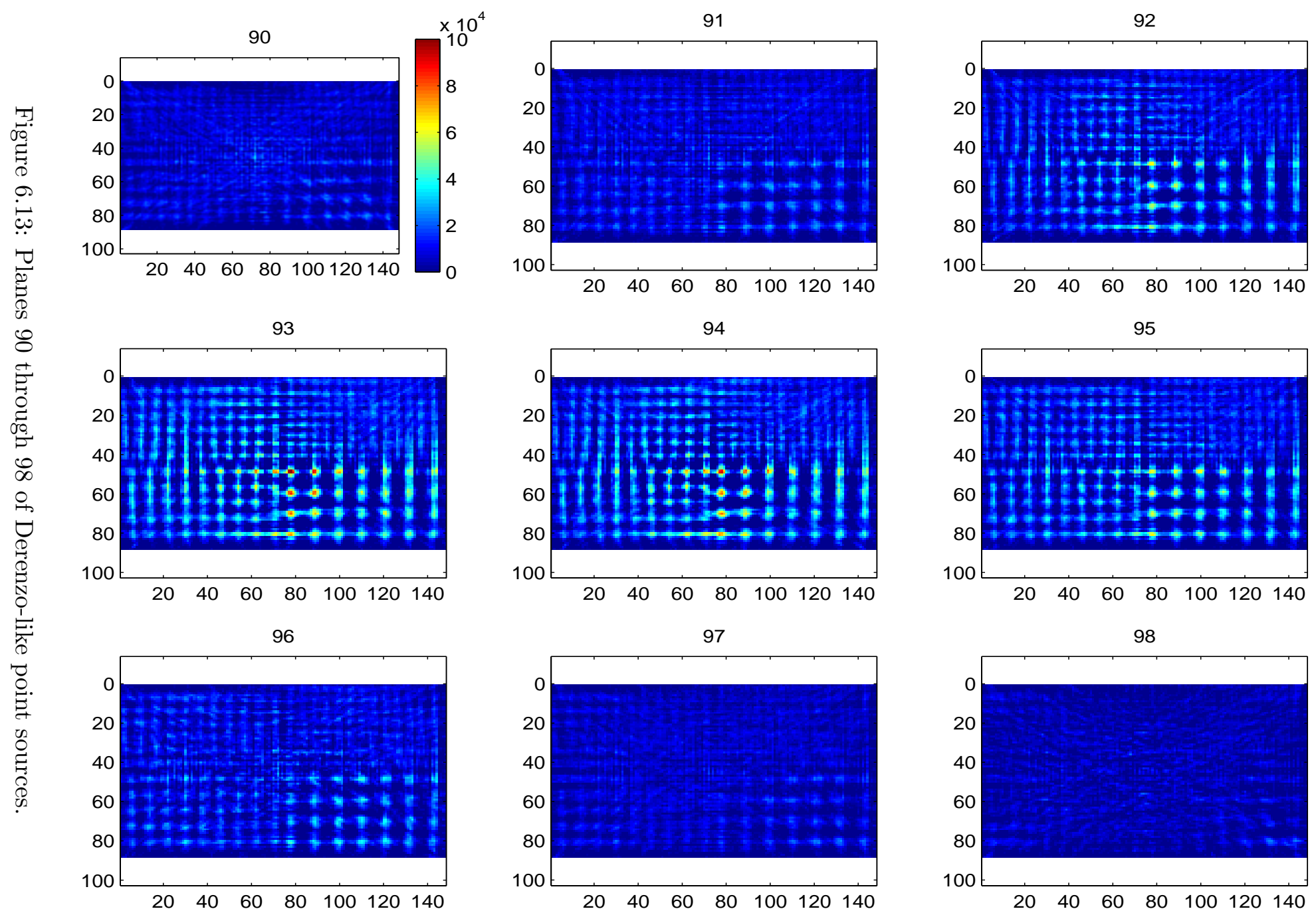


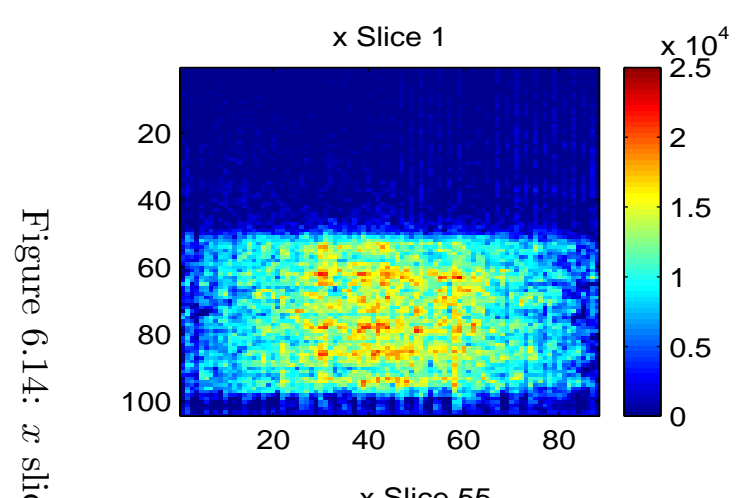

x Slice 55
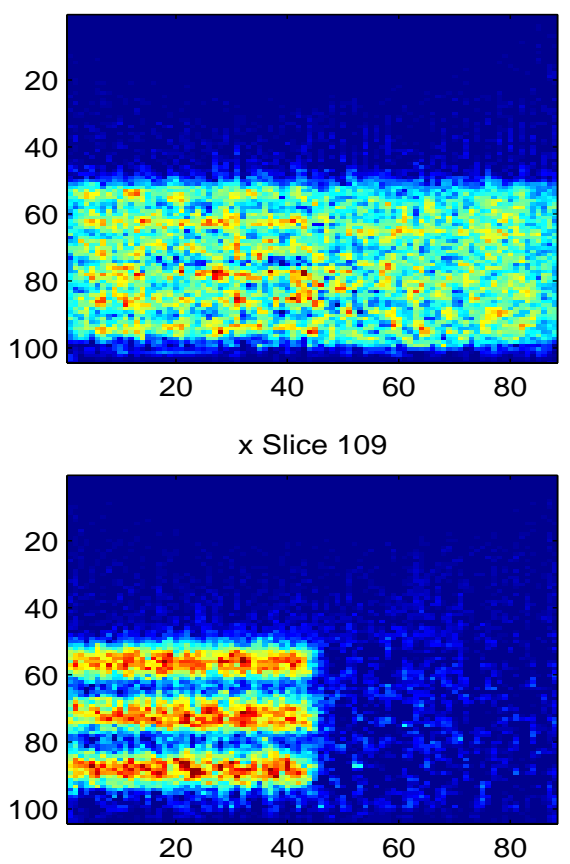

x Slice 19
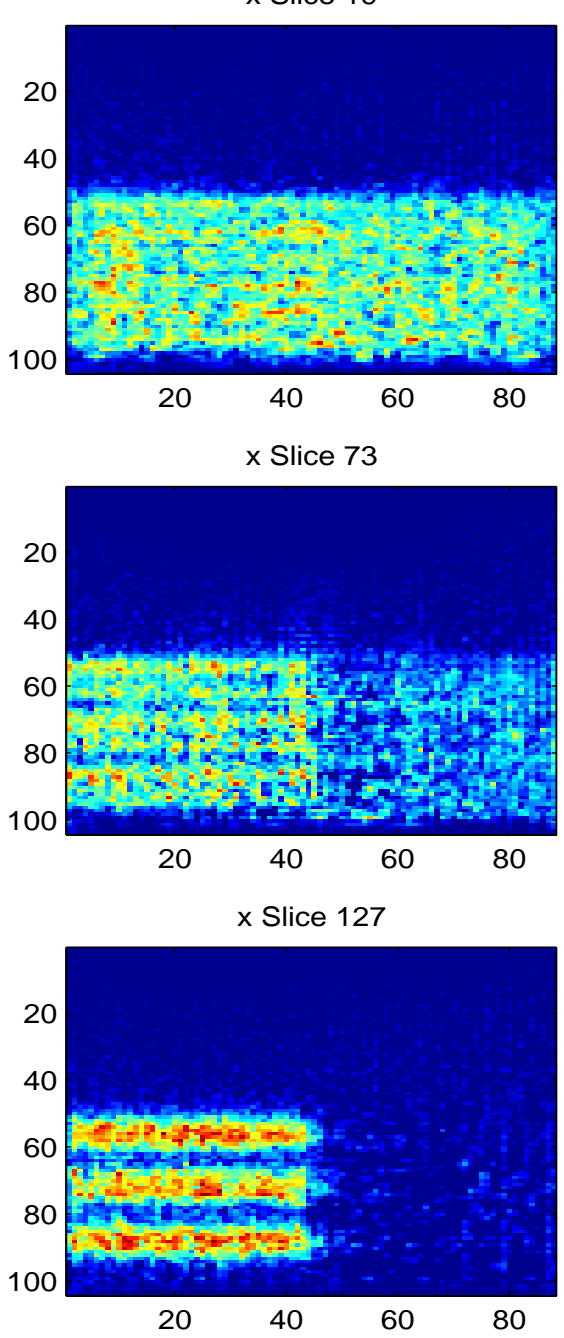

x Slice 37
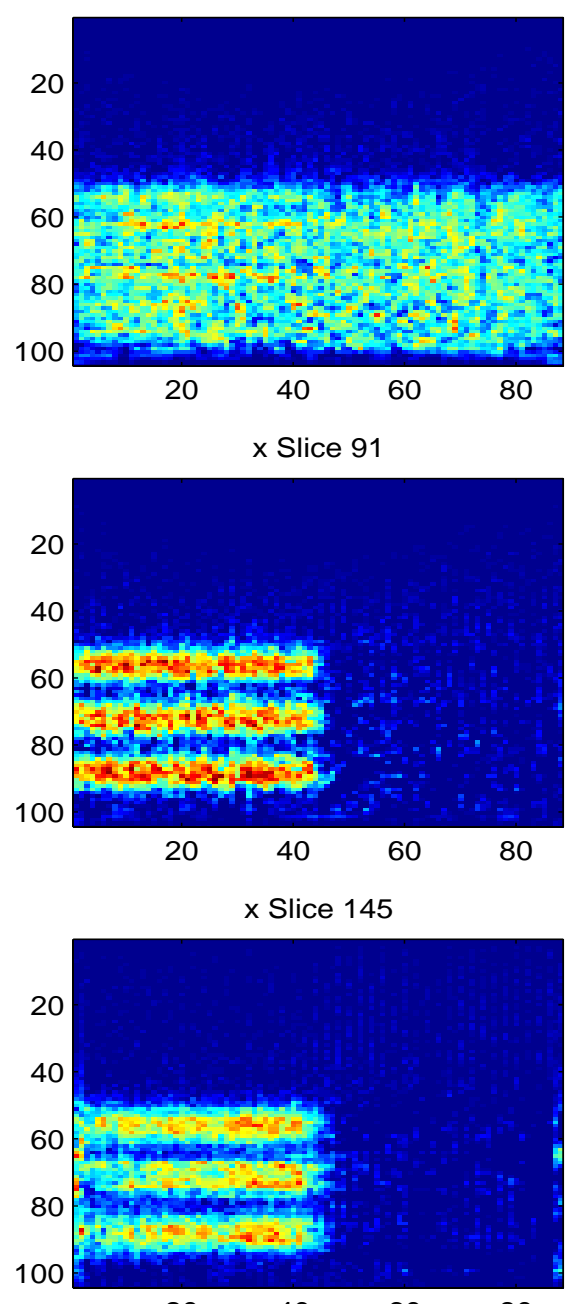

20 

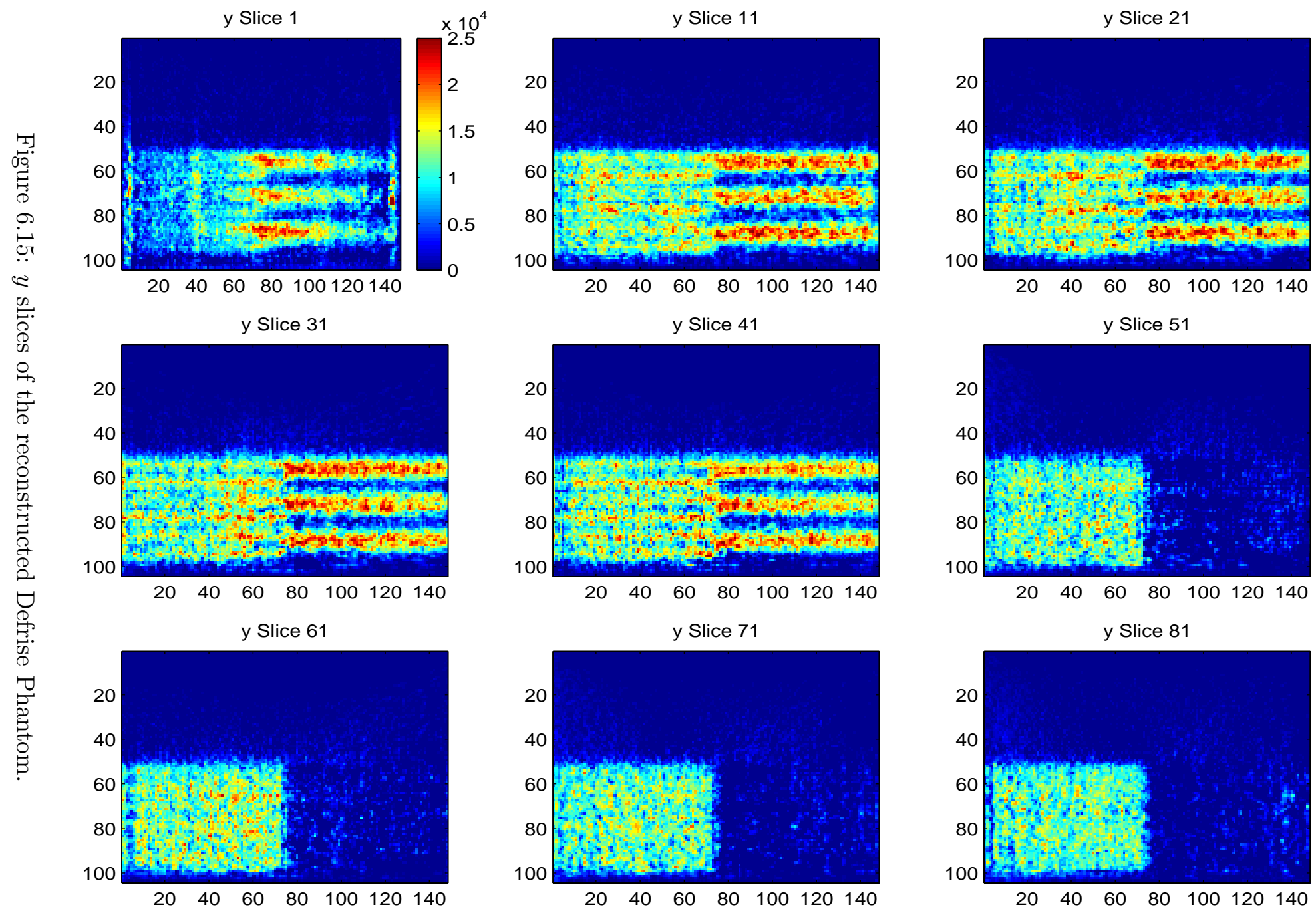

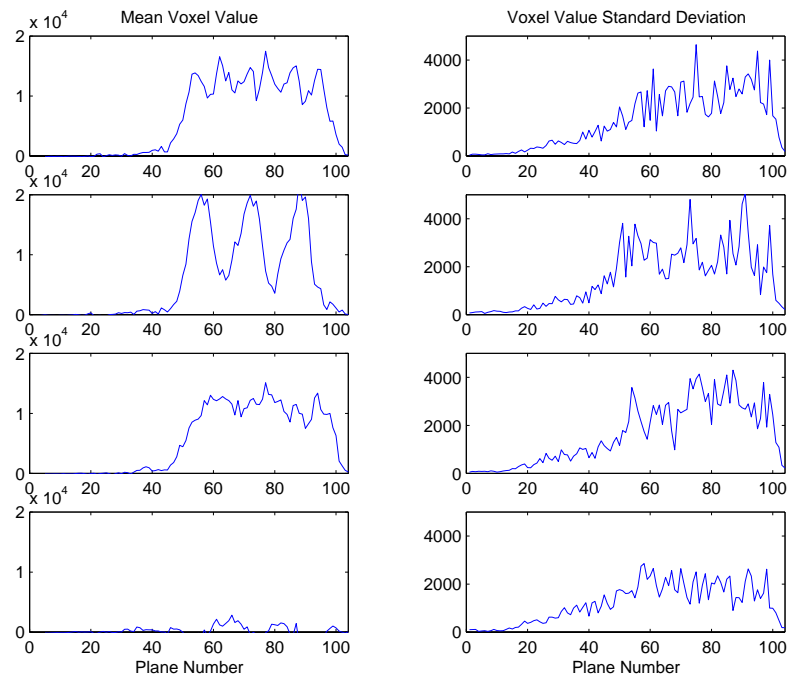

Figure 6.16: Mean Voxel Value and Standard Deviation of a $3 x 3$ voxel ROI region at the center of each quadrant of the Defrise Phantom as a function of plane number.
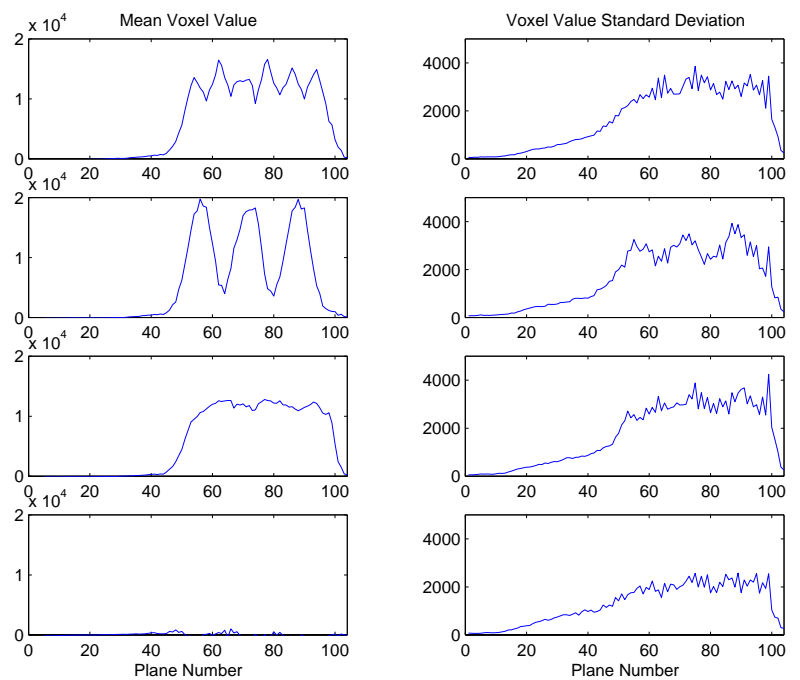

Figure 6.17: Mean Voxel Value and Standard Deviation of a $15 \times 15$ voxel ROI region at the center of each quadrant of the Defrise Phantom as a function of plane number. 


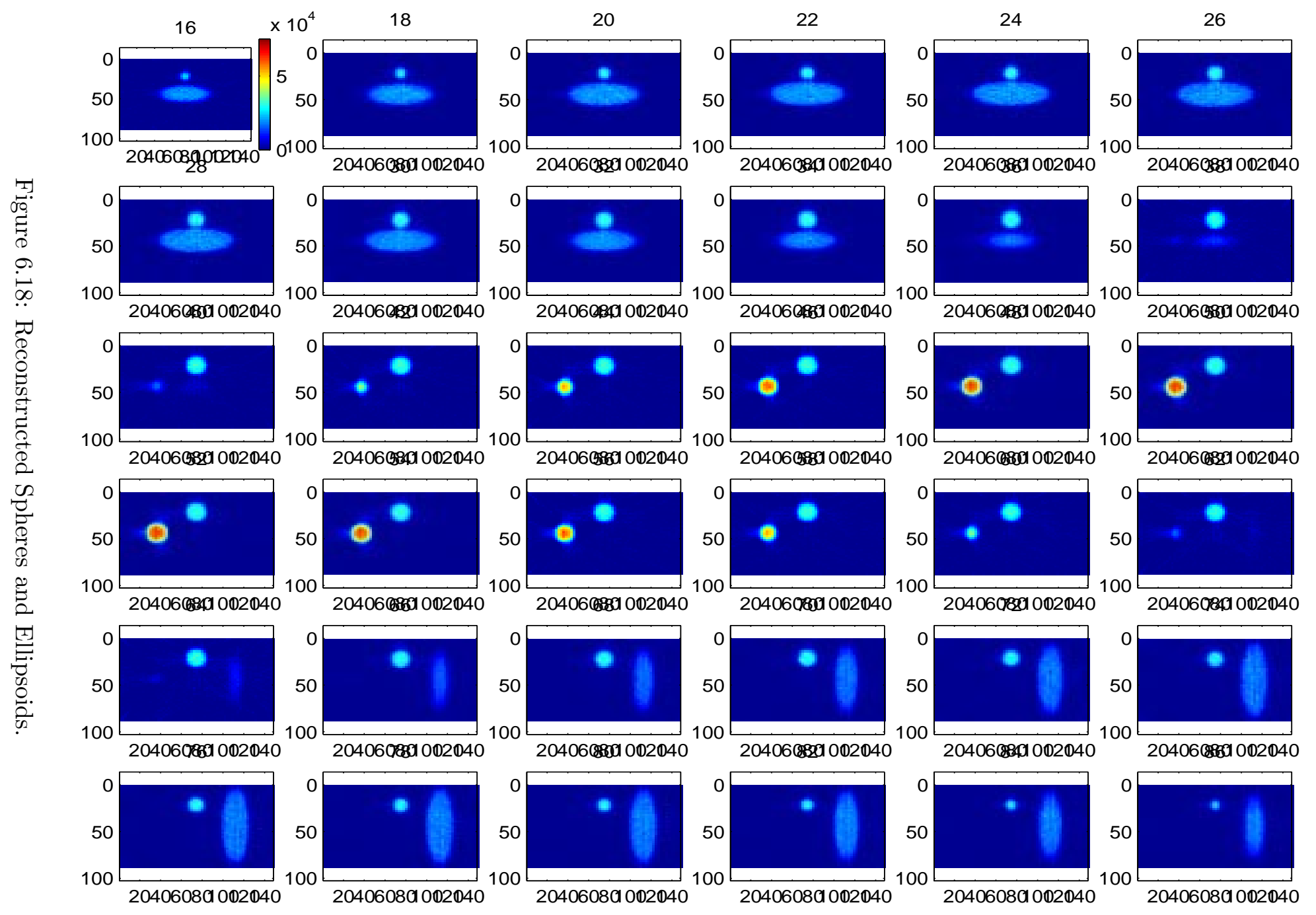




\section{Chapter 7}

\section{Conclusions and Future Directions}

In conclusion, I have developed fully 3D reconstruction procedures for data from a PEM camera with irregular sampling, missing information and depth-ofinteraction measurement capability. I use even $0.075 \mathrm{~cm}$ radial bins in the projection data to exploit the high sampling rate of the camera as well as to take advantage of fast standard algorithms that calculate Fourier transforms. I modify the standard $3 \mathrm{D}$ reconstruction algorithm to accommodate the irregularity in the angular sampling. In order to address ZEBs that result in the discretization of the DOI, I add a small random number to the measured DOI to make it approximate a continuous variable. I address the rest of the ZEBs that are due to the gaps between the modules by using the IRR algorithm that estimated the missing information by using $a$ priori information from the object. I use a simple positivity constraint as a priori information. The reconstructed images of point sources and extended phantoms are artifact-free. The point sources are essentially isotropic and have FWHMs that vary from $1.50 \mathrm{~mm}$ to $3.00 \mathrm{~mm}$ depending on its position in the FOV. I do not observe the adverse effects of radial elongation in the images. 
The algorithms that I have discussed in this dissertation show that image reconstruction can be performed with data acquired from rectangular PET cameras provided that DOI is available. It would be difficult to reconstruct images with a rectangular geometry otherwise. The lack of sampling in the problem regions in Radon space will generate artifacts in the FOV that will be difficult to correct. In particular, streak artifacts will be prominent and image resolution will severely degrade away from the center of the tomograph.

The methods that I have discussed in this thesis can be applied to other PET cameras with non-standard geometries and can measure DOI. The main problem in reconstructing images with $\mathrm{PET}$ with non-standard geometries is sampling. Properly incorporating DOI information in the reconstruction solves most of the sampling problems that arise from the geometry of the PET camera. The remaining details can be addressed in a consistent manner as I have shown here.

The simulations that I performed in this dissertation, while including many physical and realistic effects, assume a number of simplifications. For one, I have assumed scatter free media in the FOV. While this assumption is acceptable for qualitative reconstructions, scatter in the FOV needs to be simulated if quantitative information is to be extracted from the images. The simulation that I have implemented and described in this thesis allows for scatter in the FOV so that this could certainly be one of the many future directions for this project. Modeling of the generation of signals and measurement of the DOI can also be improved. I did not model crystal-to-crystal variation in the modules and thus, I have not studied its effect on the reconstructed images. The observed depth dependence in the prototype modules built by the Instrumentation Group of the CFI at LBNL exhibited some non-linearities which I did not take into account. I do not know the effects of this non-linearity in the reconstructed images as well.

There are many other issues that will need to be addressed if true image reconstruction is to be performed using data from the PEM camera. While it is true that the attenuation map of the breast does not vary as much as that of the whole 
body, the small variation in the density of the breast may necessitate further study on how attenuation correction will be performed. Assuming that the breast were homogeneous may prove insufficient. Correcting the data for scatter will also play a major part in image reconstruction with the PEM camera. Because scatter events effectively misplace an event, they can degrade the resolution of the camera if they are not handled properly. Normalization is yet another issue that I believe will be a major concern for reconstructing true data from the PEM camera. The flood source normalization data that I have consistently used throughout the dissertation will be difficult to produce due to attenuation in the medium. A more realistic normalization source may be a plane source that is tilted such that most, if not all, of the chords are activated.

Finally, there are many avenues that can be taken at this point with regards to reconstruction algorithm development. While I do not report on the $2 \mathrm{D}$ iterative studies that I have done for the PEM camera, it would certainly be interesting to see how the peculiarities and idiosyncrasies of the PEM will lend themselves to a $3 \mathrm{D}$ iterative reconstruction, either in the more conventional histogram mode or in the more recent list mode iterative techniques. In the analytic setting, it would be interesting to see the results of the FOREX algorithm applied to the PEM camera. FOREX rebins off-plane events into the proper $2 \mathrm{D}$ reconstruction planes. The rebinning is performed in Fourier space in order to take advantage of the extra information that is available in redundant 3D data. Once a complete set of consistent 2D sinograms are available, the image can be quickly reconstructed using standard $2 \mathrm{D}$ techniques. I believe that the missing information and issues that come about because of the irregular sampling in the PEM camera can be addressed in a consistent manner by the Fourier rebinning technique. 


\section{Appendix A}

\section{Fourier Rebinning Method}

The main idea behind Fourier Rebinning is that the Fourier transform of the projections at an oblique angle $\theta$ is related to the Fourier transform of the inplane projections. Let the projection data be parametrized as $p=p(s, \phi, z, \theta)$ where $s$ and $\phi$ are the familiar 2D sinogram variables, $z$ is the midpoint of the chord defining the projection point, and $\theta$ is the polar angle of the chord. Note that this is different from the parametrization that I have used throughout this dissertation. In particular, there is no notion of a projection plane in this parametrization of the projection data. I have used the direction angles $(\theta, \phi)$ to define the projection plane and two orthogonal variables $(u, v)$ to define the location of the projection point in the plane. The projection data is, however, still defined by Eqn. 5.1

$$
\mathcal{P} f\left(\mathbf{u}=\mathbf{x}^{\perp}, \omega\right)=\int_{\mathbf{R}} d t f(\mathbf{x}-t \omega) ; \quad \xi \cdot \mathbf{x}=0
$$

but now, the parametrization is

$$
[\mathcal{P} f](s, \phi, z, \theta)=\int_{\mathbf{R}} d t f(s \cos \phi-t \sin \phi, s \sin \phi+t \cos \phi, z+t \tan \theta) .
$$

The first step in FOREX is taking the Fourier transform of an off-plane projection $\mathcal{P} f(\theta \neq 0)$ in the $s$ and $z$ variables.

$$
\left[\mathcal{F}_{s} \mathcal{F}_{z} \mathcal{P} f\right]\left(k_{s}, k_{z}, \phi, \theta\right)
$$




$$
\begin{aligned}
& =\int d s e^{-i s k_{s}} \int d z e^{-i z k_{z}} \int d t f(s \cos \phi-t \sin \phi, s \sin \phi+t \cos \phi, z+t \tan \theta) \\
& =\int d s e^{-i s k_{s}} \int d t e^{-i k_{z}(-t \tan \theta)} F_{z}\left(s \cos \phi-t \sin \phi, s \sin \phi+t \cos \phi, k_{z}\right)
\end{aligned}
$$

since $\mathcal{F} f(x-a)=e^{-i k a} F(k)$. The variables $(s, t)$ are then transformed back into the Cartesian coordinate variables, $(x, y)$, by the rotation

$$
\left[\begin{array}{l}
s \\
t
\end{array}\right]=\left[\begin{array}{cc}
\cos \phi & \sin \phi \\
-\sin \phi & \cos \phi
\end{array}\right]\left[\begin{array}{l}
x \\
y
\end{array}\right]
$$

so that Eqn. A.3 can be rewritten as

$$
\begin{aligned}
& {\left[\mathcal{F}_{s} \mathcal{F}_{z} \mathcal{P} f\right]\left(k_{s}, k_{z}\right) } \\
= & \int d x \int d y e^{-i k_{s}(x \cos \phi+y \sin \phi)} e^{-i k_{z} \tan \theta(x \sin \phi-y \cos \phi)} F_{z}\left(x, y, k_{z}\right) \\
= & \int d x e^{-i x\left(k_{s} \cos \phi-k_{z} \tan \theta \sin \phi\right)} \int d y e^{-i y\left(k_{s} \sin \phi+k_{z} \tan \theta \cos \phi\right)} F_{z}\left(x, y, k_{z}\right) \\
= & F\left(k_{s} \cos \phi-k_{z} \tan \theta \sin \phi, k_{s} \sin \phi+k_{z} \tan \theta \cos \phi, k_{z}\right) .
\end{aligned}
$$

Note that the last expression is of the form $F\left(k_{x}^{\prime}, k_{y}^{\prime}, k_{z}\right)$. This is the Central Slice Theorem again. Redefining

$$
\begin{aligned}
k_{z} \tan \theta & =k_{s} \tan \sigma \\
k_{s}^{\prime} & =k_{s} \sec \sigma
\end{aligned}
$$

we have

$$
\begin{aligned}
{\left[\mathcal{F}_{s} \mathcal{F}_{z} \mathcal{P} f\right]\left(k_{s}, k_{z}\right) } & =F\left(k_{s} \cos \phi-k_{s} \tan \sigma \sin \phi, k_{s} \sin \phi+k_{s} \tan \sigma \cos \phi, k_{z}\right) \\
& =F\left(k_{s} \sec \sigma \cos (\phi+\sigma), k_{s} \sec \sigma \sin (\phi+\sigma), k_{z}\right)
\end{aligned}
$$

Taking the Fourier transform with respect to $\phi$

$$
\begin{aligned}
{\left[\mathcal{F}_{s} \mathcal{F}_{z} \mathcal{P} f\right]\left(k_{s}, k_{z}\right) } & =\int d \phi e^{-i \Phi \phi} F\left(k_{s}^{\prime} \cos (\phi+\sigma), k_{s}^{\prime} \sin (\phi+\sigma), k_{z}\right) \\
& =e^{i \Phi \sigma} \int d \eta e^{-i \Phi \eta} F\left(k_{s}^{\prime} \cos \eta, k_{s}^{\prime} \sin \eta, k_{z}\right)
\end{aligned}
$$


Note that the final expression states that

$$
\mathcal{F}_{z} \mathcal{F}_{s} \mathcal{F}_{\phi} \mathcal{P} f\left(k_{s}, k_{z}, \Phi, \theta\right)=e^{i \Phi \sigma} \mathcal{F}_{z} \mathcal{F}_{s} \mathcal{F}_{\phi} \mathcal{P} f\left(k_{s}^{\prime}, k_{z}, \Phi, 0\right)
$$

Therefore, the Fourier transform of the off-plane projections may be rebinned to the Fourier transform of the in-plane projections which may then be reconstructed in the $2 \mathrm{D}$ mode. 


\section{Bibliography}

[1] P. Strax, Editor, Control of Breast Cancer Through Mass Screening, PSG Publishing Co., Massachusetts, 1978.

[2] Strategies for Managing the Breast Cancer Research Program. National Academy of Sciences Institute of Medicing Report to the U.S. Army Medical Research and Development Command (pp. 58), 1993.

[3] S. H. Heywang-Köbrunner, I. Schreer, and D. D. Dershaw, Diagnostic Breast Imaging, Thieme, New York, 1997.

[4] W. W. Moses, Continuing Education Series Lecture Notes, IEEE Medical Imaging Conference, San Francsico, 1995.

[5] M. B. Imran, K. Kubota, S. Yamada, H. Fukuda, K. Yamada, T. Fujiwara and M. Itoh, "Lesion-to-Background Ratio in Nonattenuation-corrected Whole-Body FDG PET Images," J. Nuc. Med., 39, 1219-23, 1998.

[6] A. Lehninger, Biochemistry, Worth Publishers Inc., New York, 1975.

[7] O. Warburg, The metabolism of Tumors, Richard Smith Inc., New York, 1931.

[8] A. Fischman and N. Alpert, "FDG-PET in Oncology: There's More to It Than Looking at Pictures," J. Nuc. Med., 34, 6-11, 1993.

[9] G. D. Hutchins, "Mammographic PET (MAMPET): a dedicated imaging device for breast cancer applications," Grant proposal to the Whitaker Foundation. 
[10] C. J. Thompson, K. Murthy, Y. Picard, I. N. Weinberg, R. Mako, "Positron Emission Mammography (PEM): A Promising Technique for Detecting Breast Cancer," IEEE Trans. Nuc. Sci. NS-42, 1012-1017, 1994.

[11] R. L. Wahl, R. L. Cody, G. D. Hutchins, et al. "Primary and metastatic breast carcinoma: initial clinical evaluation with PET with the radiolabeled glucose analog 2-18F-Fluoro-2-Deoxy-D-glucose," Radiology 179, 756-770, 1991.

[12] W. W. Moses, P. R. G. Virador, S. E. Derenzo, R. Huesman and T. Budinger, "Design of a High-Resolution, High-Sensitivity PET Camera for Human Brains and Small Animals," IEEE Trans. Nuc. Sci., NS-44, 1487-1491, 1997.

[13] J. S. Karp and M. Daube-Witherspoon, "Depth of interaction determination in $\mathrm{NaI}(\mathrm{Tl})$ and BGO scintillation crystals usin a temparature gradient," Nuc. Inst. Meth., A-260, 509-517, 1987.

[14] J. G. Rogers, C. Moisan, E. M. Hoskinson et al. "A practical block detector for a depth-encoding PET camera," IEEE Trans. Nuc. Sci., NS-43, pp. 732-738, 1991.

[15] W. W. Moses, S. E. Derenzo, C. L. Melcher et al. "A room temparature LSO/PIN photodiode PET detector module that measures depth of interaction," IEEE Trans. Nuc. Sci., NS-42, 1085-1089, 1995.

[16] W. Moses, R. Huesman and S. Derenzo, "A new algorithm for using depth-ofintermation measurement information in PET data acquisition," J. Nuc. Med., 32, 995, 1991.

[17] William Press, Saul Teukolsky, William Vetterling, Brian Flannery, Numerical Recipes in C, 576, Cambridge Press, New York, 1995.

[18] J. S. Karp, A. J. Becher, S. Matej, and P. E. Kinahan, "Data processing and image reconstruction methods for the HEAD PENN-PET scanner," IEEE Trans. Nuc. Sci., NS-45, 1144-51, 1998. 
[19] M. H. Buonocore, W. R. Brody, and A. Macovski, "A natural pixel decomposition for two dimensional image reconstruction," IEEE Trans. Biomed. Eng., BME-28, 69-78, 1981.

[20] J. A. Weir, Lebesgue Integration and Measure, Cambridge University, Cambridge, 1973.

[21] P. Bartzakos and C. J. Thompson, "A depth-encoded PET detector," IEEE Trans. Nuc. Sci., NS-38, 732-738, 1991.

[22] P. A. Rattey and A. G. Lindgren, "Sampling the 2-D Radon Transform," IEEE Trans. Acoust., Speech, Signal Process, ASSP-29, 994-1002, 1981.

[23] S. Deans, The Radon Transform and Some of its Applications, John Wiley and Sons, New York, 1983.

[24] R. N. Bracewell, "Strip integration in radio astronomy," Aust. J. Phys. 9, 198$217,1956$.

[25] J. Radon, "Über die Bestimmung von Funktionen durch ihre Integralwerte längs gewisser Mannigfaltigkeiten," Berichte Sächsische Akademie der Wissenschaften Leipzig, Math.-Phys. Kl., 69, 262-267, 1917.

[26] F. Natterer, The Mathematics of Computerized Tomography, 14, John Wiley and Sons, New York, 1986.

[27] P. V. C. Hough Method and means for recognizing complex patters, U.S. Patent 3069 654, 1962.

[28] G. Strang, Linear Algebra and its Applications, pp. 153-157, Harcourt Brace Jovanovich, San Diego, 1988.

[29] E. Lehmann, Theory of Point Estimation, Wiley, New York, 1983. 
[30] J. Ruanaidh and W. Fitzgerald Statistics and Computing, Springer, New York, 1996.

[31] J. Fessler, "Penalized Weighted Least-Squares Image Reconstruction for Positron Emission Tomography," IEEE Trans. Med. Imag., MI-13, 290-300, 1994.

[32] E. J. Hoffman, S. C. Huang, M. J. Phelps, and D. E. Kuhl, "Quantitation in positron emission computed tomography: 4. Effect of accidental coincidences," J. Comput. Ass. Tom., 5-3, 391-400, 1981.

[33] R. Billingley, Probability and Measure, Wiley, New York 1986.

[34] R. H. Huesman, G. T. Gullberg, W. L. Greenberg, and T. F. Budinger, RECLBL library user's manual, Lawrence Berkeley National Laboratory, Berkeley, 1977.

[35] R. H. Huesman, G. J. Klein, and W. W. Moses, J. Qi, B. W. Reutter, and P. R. G. Virador, "List Mode Maximum Likelihood Reconstruction Applied to Positron Emission Mammography with Irregular Sampling," Lawerence Berkeley National Laboratory Technical Report LBNL-44259, Berkeley, CA, 1999.

[36] J. Fessler, Continuing Education Series Lecture Notes, IEEE Medical Imaging Conference, Toronto, 1998.

[37] J. Qi and R. Leahy, "Resolution and noise properties of MAP reconstruction for fully $3 \mathrm{D}$ PET," submitted to IEEE-TMI.

[38] H. H. Barrett, D. W. Wilson and B. W. Tsui, "Noise properties of the EM algorithm: I. Theory," Phys. in Med. and Bio., 39, 833-846, 1994.

[39] W. Wang and G. Gindi, "Noise analysis of MAP-EM algorithms for emission tomography," Phys. in Med. and Bio., 42, 2215-2232, 1997. 
[40] E. J. Hoffman, M. E. Phelps, G. Wisenberg, H. R. Schelbert, and D. E. Kuhl, "Electrocardiographic Gating in Positron Emission Computed Tomography," J. of Comp. Assist. Tom. 3(6), 733-739, 1979.

[41] E. Hoffman, M. Phelps Positron Emission Tomography: Principles and Quantitation, 237-286, Raven Press, New York, 1986.

[42] R. H. Huesman, E. M. Salmeron, J. R. Baker, "Compensation for Crystal Penetration in High Resolution Positron Tomography," IEEE Trans. Nuc. Sci., NS-36, 1100-1107, 1989.

[43] G. N. Ramachandran and A. V. Lakshminarayanan, Proc. Natl. Acad. Sci. US, 2236-2240, 1971.

[44] N. Keller and L. Lupton, "PET Detector Ring Aperture Function calculations using Monte Carlo techniques," IEEE Trans. Nuc. Sci., NS-30, 676-681, 1983.

[45] C. Carrier, C. Martel, D. Schmitt, R. Lecomte "Design of a high resolution positron emission tomograph using sold state scintillator crystals," IEEE Trans. Nuc. Sci., NS-35, 685-690 1988.

[46] R. A. Brooks, V. J. Shank, W. S. Friauf, S. B. Leighton, H. E. Casio and G. Di Chiro, "Design considerations for positron emission tomography," IEEE Trans. Biomed. Eng.,, BME-28 158-177, 1981.

[47] S. E. Derenzo "Initial characterization of a BGO-photodiode detector for high resolution positron emission tomography," IEEE Trans. Nuc. Sci., NS-31, 620$626,1984$.

[48] J. Seidel, J. J. Vaquero, S. Siegel, W. R. Gandler, and M. V. Green, "Depth Identification Accuracy of a Three Layer Phoswhich PET Detector Module," IEEE Trans. Nuc. Sci., NS-46, 485-490, 1999. 
[49] C. L. Melcher and J. S. Schweitzer, "Cerium-doped Lutetium Oxyorthosilicate: A Fast, Efficitne New Scintillator" IEEE Trans. Nuc. Sci., NS-39, 502-505, 1992.

[50] M. Palmer, M. Bergstrom, M. Beddoes, and B. Pate, "Effects of detector wobble motion on image noise in positron emission tomography," IEEE Trans. Med. Imag. MI-4, 58-62, 1985.

[51] J. Felsche, The crystal chemistry of the rare-earth silicates in Structure and Bonding V13 Springer-Verlag, 99-197, 1973.

[52] R. D. Evans, The Atomic Nucleus, Krieger, New York, 1982.

[53] W. W. Moses, E. Beuville and M. H. Ho, “'A Winner-Take-All' IC for determining the crystal of interaction in PET Detectors," IEEE Trans. Nuc. Sci. NS-43, 1615-1618, 1996.

[54] J. S. Huber, W. W. Moses, M. S. Andreaco, M. Loope, C. L. Melcher, and R. Nutt, "Geometry and surface treatment dependence of the light collection from LSO crystals," Nuc. Ins. and Meth. in Phys. Res. A 437, 374-380, 1999.

[55] W. W. Moses and S. E. Derenzo "Design studies for a PET detector module using a PIN photodiode to measure Depth of Interaction'," IEEE Trans. Nuc. Sci., NS-41, 1441-1445, 1994.

[56] J. S. Huber, W. W. Moses and P. R. G. Virador "Calibration of a PET detector module that measures depth of interaction," IEEE Trans. Nuc. Sci., NS-45, 1268-1272, 1998.

[57] G. Knoll, Radiation Detector and Measurement, 44-45, John Wiley and Sons, New York, 1989.

[58] M. Lederer and V. Shirley, Editors Table of Isotopes, rth Edition, 26, John Wiley and Sons, New York, 1978. 
[59] S. F. Haber, S. E. Derenzo and D. Uber, "Application of Mathematical Removal of Positron Range Blurring in Positron Emission Tomography," IEEE Trans. Nuc. Sci., 37, 1293-1299.

[60] O. Klein and Y. Nishina, "Uber die Streuung von Strahlung durch freie Elektronen nach der neuen relativistischen Quantendynamik von Dirac," Z. Physik. $\mathbf{5 2}, 853-868$.

[61] E. F. Plechaty, D. E. Cullen, and R. J. Howerton, Tables and graphs of photoninteraction cross sections from $0.1 \mathrm{keV}$ to $100 \mathrm{MeV}$ derived from the $L L N L$ Evaluated-Nuclear-Data library Lawrence Livermore National Laboratory Report \#UCRL 50400, 6, Rev. 2, 1978.

[62] K. A. Comanor, P. R. G. Virador and W. W. Moses, "Algorithms to Identify Detector Compton Scatter in PET Modules," IEEE Trans. Nuc. Sci., NS-43, 2213-2218, 1996.

[63] G. Muehllehner, J. S. Karp, and A. Guvenis, "A method for reconstructing images from data obtained with a Hexagonal Bar Positron Camera," IEEE Trans. Med. Imag. MI-4, 134-138, 1985.

[64] R. H. Huesman, S. E. Derenzo and T. F. Budinger, "A two position sampling scheme for positrion emission tomography," Nuclear Medicine and Biology, Proceedings of the Third World Congress of Nuc. Sci. and Bio., 1, 542-545, 1982.

[65] A. Dagher and C. J. Thompson, "Real-time rebinning in PET to obtain uniformly sampled projections," IEEE Trans. Nuc. Sci., NS-32, 811-817, 1985.

[66] J. S. Huber, W. W. Moses, S. E. Derenzo, M. Ho, M. S. Andreaco, M. J. Paulus, and R. Nutt, "Characterization of a 64 Channel PET Detector Using Photodiodes for Crystal Identification," IEEE Trans. Nuc. Sci. NS-44, 11971201, 1997. 
[67] B. P. Medoff, W. R. Brody, M. Nassi, and A. Macovski, "Iterative Convolution Backprojection Algorithms for Image Reconstruction from Limited Data," $J$. Opt. Soc. Am., 71-11 1493-1500, 1983.

[68] J. G. Colsher, "Fully three-dimensional emission tomography," Phys. Med. Bio., 25-1, 103-115, 1980.

[69] F. B. Atkins, G. Muehllehner and P. V. Harper, Proc. $5^{\text {th }}$ Int. Conf. on Information and Processing in Medical Imaging Oak Ridge National Laboratory, Jun 1977, Nashville TN.

[70] R. Bracewell, The Fourier Transform and Its Applications, 251-253, McGraw Hill, New York, 1965.

[71] M. Abramowitz and I. Segun, Handbook of Mathematical Functions, 487, Dover, New York, 1968.

[72] P. E. Kinihan and J. G. Rogers, "Analytic 3D Image Reconstruction Using All Detected Events," IEEE Trans. Nuc. Sci., NS-36, 964-968, 1989.

[73] K. Erlandsson, P. D. Esser, S. E. Strand and R. L. Heertum, "3D reconstruction for a multi-ring PET scanner by single-slice rebinning and axial deconvolution," Phys. Med. Bio., 39, 619-629, 1994.

[74] R. M. Lewitt, G. Muehllehner, and J. S. Karp, "Three-dimensional reconstruction in PET my multi-slice rebinning and axial image filtering," Phys. Med. Bio., 39, 321-340, 1994.

[75] M. Defrise, P. E. Kinihan, D. W. Townsend, C. Michel, M. Sibomana and D. Newport, "Exact and approximate rebinning algorithms for 3D PET data," IEEE Trans. Med. Imag., MI-16, 145-158, 1997. 
[76] L. Xuan, M. Defrise, C. Michel, M. Sibomana, C. Comtat, P. Kinihan, D. Townsend, "Exact rebinning methods for 3D PET," IEEE Trans. Med. Imag. MI-18, 657-64, 1999.

[77] S. E. Derenzo, R. H. Huesman, J. L. Cahoon, A. B. Geyer, W. W. Moses, D. C. Uber, T. Vuletich, and T. F. Budinger, "A positron tomograph with 600 BGO crystals and $2.6 \mathrm{~mm}$ resolution," IEEE Trans. Med. Imag., NS-35, 659-664, 1988. 